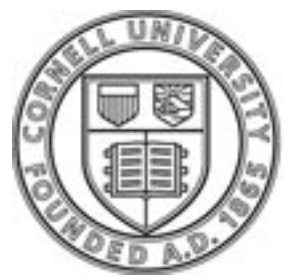

\title{
Length Scaling in Spacecraft Dynamics
}

by Justin Allen Atchison

This thesis/dissertation document has been electronically approved by the following individuals:

Peck,Mason (Chairperson)

Erickson,David (Minor Member)

Campbell,Mark (Minor Member) 
LENGTH SCALING IN SPACECRAFT DYNAMICS

\author{
A Dissertation \\ Presented to the Faculty of the Graduate School \\ of Cornell University \\ In Partial Fulfillment of the Requirements for the Degree of \\ Doctor of Philosophy
}

by

Justin Allen Atchison

August 2010 
(C) 2010 Justin Allen Atchison 


\title{
LENGTH SCALING IN SPACECRAFT DYNAMICS
}

\author{
Justin Allen Atchison, Ph. D.
}

Cornell University 2010

This research evaluates the length-dependence of a number of space environmental accelerations, both orbital and angular. Many non-gravitational effects accelerate a smaller body more than a larger body, thanks to ratios such as area-to-mass that vary inversely with characteristic length. This research studies these accelerations, and the corresponding dynamics, with an interest in applying the results to methods of propellant-free spacecraft propulsion. After surveying space environmental accelerations, the analysis focuses on three particular cases: solar radiation pressure, aerodynamic drag, and the Lorentz force. Each of these accelerations has an explicit dependence on length-scaling, such that millimeter-scale bodies experience characteristically larger magnitudes of acceleration than typical spacecraft.

For the case of solar-radiation pressure, a flat integrated circuit is considered as a low-cost, feasible solar sail with passive, locally and/or globally stable attitude control. The modified orbital and attitude dynamics are considered for heliocentric, geocentric, and three-body orbits.

For aerodynamic drag, a similar thin-plate integrated circuit bus is considered for atmospheric re-entry. Here, the spacecraft's cross-sectional area-to-mass ratio drives the magnitude of drag. So, small bodies can remove orbital kinetic energy very efficiently. Further, length-scaling laws for thermodynamics and fluid mechanics show that a very small spacecraft can even survive the intense re-entry thermal environment without burning-up or requiring active control. 
Research on the Lorentz force has found that an orbiting body with an electrostatic charge can interact with a planetary magnetic field and experience a force. In this case, the driving parameter is the electrostatic charge-to-mass ratio, a quantity that depends on the inverse square of characteristic length. This analysis presents a proposal for a small spacecraft that can demonstrate the Lorentz force in Earth orbit. A sample low charge-to-mass mission is proposed, wherein the Lorentz force is considered for Jovian capture and orbit circularization. The Lorentz force is also evaluated in relation to the so-called Earth Flyby Anomaly, in which an unknown acceleration affected the orbit of six spacecraft as they were executing Earth gravity assists. This research finds that the Lorentz force cannot be associated with the unknown acceleration, in spite of having similar characteristics. 


\section{BIOGRAPHICAL SKETCH}

Justin Allen Atchison was born in 1983 in Shreveport, Louisiana to Bob and Pam Atchison. After graduating from Caddo Magnet High School, Justin entered Louisiana Tech University in the fall of 2001. There he earned a Bachelor of Science in Mechanical Engineering as a Presidential Scholar in 2005. Justin then began graduate study at Cornell University where he joined Dr. Mason Peck’s Space Systems Design Studio. There he received a National Defense Science and Engineering Graduate Fellowship and a National Science Foundation Fellowship. He was awarded a Master of Science in Aerospace Engineering March 2008. Following graduation, he will begin his professional career at the Johns Hopkins University Applied Physics Laboratory. 
To my parents. 


\section{ACKNOWLEDGMENTS}

I would like to first thank my graduate adviser Professor Mason Peck of Cornell University. Mason has freely shared his insights, wisdom, and clever rules-of-thumb. He always expected my best efforts and always led by example. Mason has been an ideal graduate adviser and it has been a privilege to be one of his students.

I'd like to acknowledge my graduate committee: Dr. Mark Campbell and Dr. David Erickson of Cornell University. I've appreciated all of their help and insight as I've moved forward in a new research domain.

I thank Marcia Sawyer, Cornell University's Mechanical and Aerospace Engineering Graduate Field Administrator. Marcia has been a joy to know, and I will forever consider her a model Ithacan, which is a high compliment by my reckoning.

I'd also like to thank all of the members of the Space Systems Design Studio of Cornell University, namely Brett Streetman, Michele Carpenter, Daniel Brown, Joseph Shoer, Michael Norman, Laura Jones, Jessica Gersh, and Rodrigo Zeledon. In particular, Brett Streetman was incredibly helpful, patient, and encouraging as I was first beginning my graduate research. Brett has been a great example to follow, as well as a colleague and close friend.

I'd like to thank the incredible efforts and progress offered by fellow students who participated in the Sprite spacecraft team throughout my tenure: Zachary Manchester, Phillipe Tosi, Ryan Zhou, Matt Blair, Alfred Ernst, Brad Lyon, Victoria Alexander, Sharon Kotz, and Parker Imrie. In particular, Zachary Manchester has invested his sizeable technical expertise and energy into the project, and I'm incredibly pleased that he will be continuing this research.

I'd like to thank my colleagues and friends in Cornell University's Mechanical and Aerospace Engineering graduate program who began their graduate programs with me 
in 2005, notably Edgar Cuji, Bernardo Cordovez, Michael Tolley, and Maureen Lynch. I value their friendship and camaraderie, and I believe that we succeeded as a team, thanks in large part to mutual support and encouragement.

I'm indebted to my wonderful family, whose support has been the foundation of all of my successes. I thank my parents, Bob and Pam Atchison, who have held me to a high standard and then worked ceaselessly to help me reach it. Their love has been a constant in my life, helping me to stay positive in spite of long winters, tough exams, and what I'll call "the trials of gaining wisdom." I thank my brother and sister-in-law, Jarrod and Rebecca Atchison, who have always been a source of encouragement, morale, and perspective. I'll always look up to Jarrod as my role model, and I've benefited immeasurably from attempting to follow his example. He’s always chosen to be the protective and proud older brother, freely offering his considerable (and considerate) guidance and wisdom, and I am especially thankful.

Finally, I sincerely thank my loving fiancé, and soon-to-be wife, Lisa Toth. Lisa’s caring smile and warm heart have given meaning to many of my efforts. While I look forward to the next steps in our lives together, I will always cherish these past years as the time I first met and came to know such a wonderful person. 


\section{TABLE OF CONTENTS}

Biographical Sketch......................................................................................ii

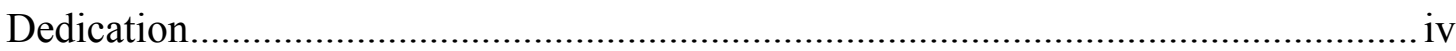

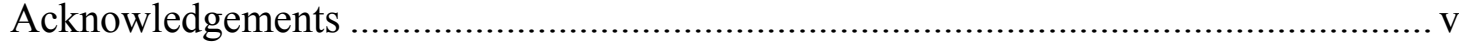

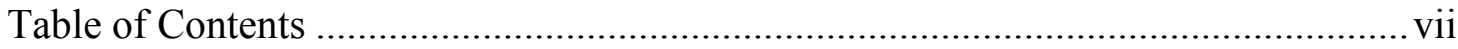

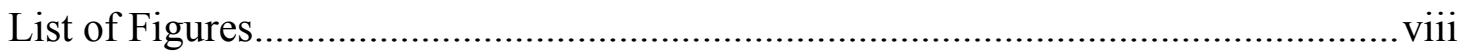

List of Tables .............................................................................................

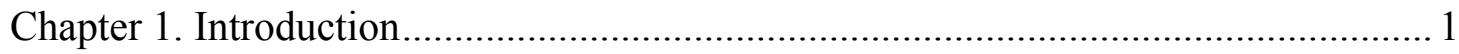

Chapter 2. A Survey of Length Scaling in Spacecraft Dynamics ............................... 7

Chapter 3. A Passive, Sun-Pointing, Millimeter-Scale Solar Sail................................ 60

Chapter 4. Microscale Atmospheric Re-Entry Sensors ........................................... 103

Chapter 5. A Millimeter-Scale Lorentz-Propelled Spacecraft ................................ 130

Chapter 6. Lorentz Augmented Jovian Orbit Insertion ......................................... 174

Chapter 7. Lorentz Accelerations in the Earth Flyby Anomaly .............................. 196

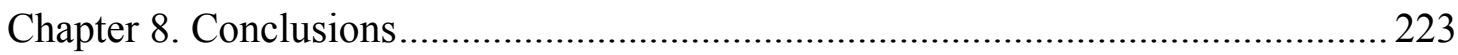




\section{LIST OF FIGURES}

Figure 2.1 Characteristic length for geometries of interest ....................................... 15

Figure 2.2 Angle of rotation associated with gravity gradient torque ......................... 18

Figure 2.3 Orientation of lift and drag forces on a flat plate in fluid flow ................... 21

Figure 2.4 Drag and lift coefficients vs angle-of-attack for a flat plate ....................... 22

Figure 2.5 Pitch angle and vectors associated with solar radiation pressure................. 22

Figure 2.6 Direction of Lorentz force in a retrograde elliptical Earth orbit .................. 32

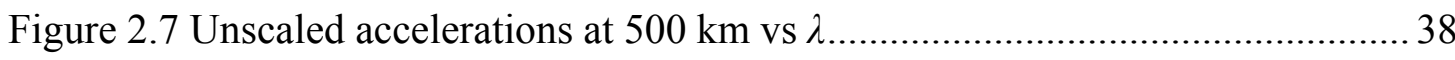

Figure 2.8 Unscaled accelerations at $1,000 \mathrm{~km}$ vs $\lambda$..................................................... 38

Figure 2.9 Unscaled accelerations at $10,000 \mathrm{~km}$ vs $\lambda$................................................. 38

Figure 2.10 Accelerations for a sphere, cube, and plate at $500 \mathrm{~km}$ vs $\lambda$.................... 38

Figure 2.11 Accelerations for a sphere, cube, and plate at $1,000 \mathrm{~km}$ vs $\lambda$.................. 39

Figure 2.12 Accelerations for a sphere, cube, and plate at $10,000 \mathrm{~km}$ vs $\lambda$................ 39

Figure 2.13 Unscaled angular accelerations at $500 \mathrm{~km}$ vs $\lambda$....................................... 41

Figure 2.14 Unscaled angular accelerations at $1,000 \mathrm{~km}$ vs $\lambda$...................................... 41

Figure 2.15 Unscaled angular accelerations at 10,000 km vs $\lambda$................................... 41

Figure 2.16 Angular accel. for a sphere, cube, and plate at $500 \mathrm{~km}$ vs $\lambda$.................... 41

Figure 2.17 Angular accel. for a sphere, cube, and plate at $1,000 \mathrm{~km}$ vs $\lambda$................ 42

Figure 2.18 Angular accel. for a sphere, cube, and plate at $10,000 \mathrm{~km}$ vs $\lambda \ldots \ldots \ldots \ldots \ldots . . .42$

Figure 2.19 Accelerations acting on a candidate spacecraft vs altitude ....................... 43

Figure 2.20 Angular accel. acting on a candidate spacecraft vs altitude....................... 43

Figure 2.21 Time-history of altitude and temperature for re-entry .............................. 48

Figure 2.22 Equilibrium potentials for a battery in a vacuum and plasma....................50

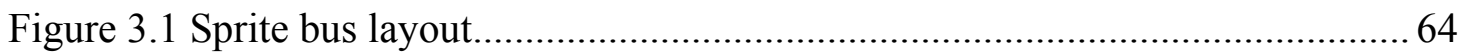

Figure 3.2 Sample values of exposed area to mass for dust and solar sails .................. 69

Figure 3.3 Simple circular maneuvers enabled by sun-pointing attitude ...................... 74

Figure 3.4 Earth fixed time-history of a geocentric spacecraft with SRP ..................... 77

Figure 3.5 Sun-Earth fixed time-history of a geocentric spacecraft with SRP ............ 77

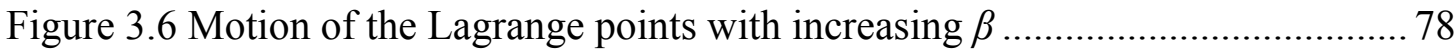

Figure 3.7 Estimated CME warning time from L1 with increasing $\beta$........................ 80 
Figure 3.8 Stable, sun-pointing corner-cube architecture ......................................... 81

Figure 3.9 Faceted surface viewed from top and isometric viewpoints ....................... 82

Figure 3.10 SRP induced torque as a function of pitch angle ...................................... 85

Figure 3.11 Attitude time-history for faceted surface with initial deviation.................. 85

Figure 3.12 Anisotropic etching and coating process …………………….............. 86

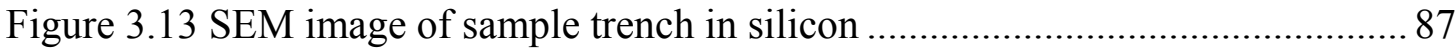

Figure 3.14 SEM image of a coated sample trench in silicon ........................................ 87

Figure 3.15 A 6 loop, $240 \mu \mathrm{m}$ wide octagonal inductor................................................. 87

Figure 4.1 Spacecraft-on-a-Chip square flat plate geometry ....................................... 106

Figure 4.2 Three dimensional orientation of lift and drag on flat plate ..................... 107

Figure 4.3 Two dimensional orientation of lift and drag on flat plate ....................... 107

Figure 4.4 Drag and lift coefficients vs angle-of-attack for a flat plate ...................... 109

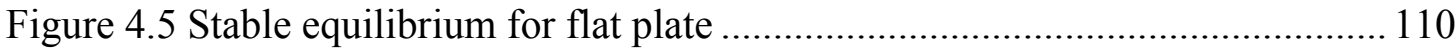

Figure 4.6 Maximum temperature during re-entry for face-on attitude ...................... 117

Figure 4.7 Maximum temperature during re-entry for edge-on attitude ..................... 117

Figure 4.8 Altitude time-history for face-on re-entry................................................. 119

Figure 4.9 Drag and velocity time-histories for face-on re-entry ................................ 119

Figure 4.10 Temperature time-history for face-on re-entry ....................................... 119

Figure 4.11 Knudsen and Reynolds time-histories for face-on re-entry ..................... 119

Figure 4.12 Altitude time-history for edge-on re-entry ............................................. 120

Figure 4.13 Velocity time-history for edge-on re-entry ........................................... 120

Figure 4.14 Drag time-history for edge-on re-entry ................................................. 120

Figure 4.15 Temperature time-histories for edge-on re-entry ..................................... 120

Figure 4.16 Knudsen number time-history for edge-on re-entry ............................... 121

Figure 4.17 Reynolds number time-history for edge-on re-entry................................ 121

Figure 4.18 Current large-scale PCB prototype …………........................................ 121

Figure 4.19 Power subsystem performance in a sun-simulator ................................ 123

Figure 4.20 Filtered DC power subsystem output ……………………………....... 123

Figure 5.1 Vector depiction of the Lorentz force in a prograde,circular orbit ............ 137

Figure 5.2 Magnitude of Lorentz acceleration in a circular orbit................................ 137

Figure 5.3 Deviations from circular Keplerian orbits by charged spacecraft.............. 139

Figure 5.4 Arc separation rate in a prograde circular orbit at $350 \mathrm{~km}$....................... 139 
Figure 5.5 Illustration of plasma potential profile. 142

Figure 5.6 Sample NASCAP static plasma potential ............................................... 146

Figure 5.7 Linear charge density and sheath size for a filament ............................... 150

Figure 5.8 Equilibrium potentials for Polonium 210............................................... 154

Figure 5.9 Solution flow for a differentially charged body in ram currents................ 161

Figure 5.10 Solution flow for a differentially charged body in OML currents .......... 161

Figure 5.11 Contours of charge-to-mass for a mesh sphere ...................................... 162

Figure 5.12 Conceptual circuit diagram for communications system......................... 163

Figure 5.13 Conceptual RF pulsed output signal ..................................................... 163

Figure 5.14 Piezoelectric crystal equivalent-analog circuit ....................................... 164

Figure 5.15 FCC allocated bands and atmospheric attenuation ................................. 165

Figure 5.16 Illustration of differentially charged attitude motion ............................... 167

Figure 5.17 Illustration of a sample spacecraft architecture........................................ 169

Figure 6.1 Vector depiction of Lorentz force in an elliptic,retrograde orbit............... 178

Figure 6.2 Orbit time-histories for parabolic approaches........................................... 181

Figure 6.3 Energy time-histories for parabolic approaches....................................... 181

Figure 6.4 Eccentricity time-histories for parabolic approaches................................. 182

Figure 6.5 Orbit time-histories with bang-off charge................................................ 183

Figure 6.6 LAO captures for a range of initial conditions and charges ....................... 185

Figure 6.7 Time-to-capture as a function of charge-to-mass ....................................... 189

Figure 6.8 Minimum charge-to-mass capture …………………………………...... 189

Figure 6.9 Radius time-history for bang-off actuated charge..................................... 190

Figure 6.10 Eccentricity time-history for bang-off actuated charge ............................ 190

Figure 7.1 Direction of $v_{r e l} \mathrm{x} B$ for the NEAR EGA ………..................................... 204

Figure 7.2 Normalized magnitude of $v_{\text {rel }} \times B$ for the NEAR EGA..............................205

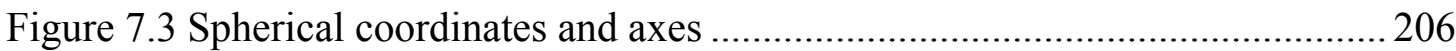

Figure 7.4 Accumulated change in orbital elements for positive charge ................... 208

Figure 7.5 Diagram of state definitions .................................................................. 210

Figure 7.6 Sample functions approximated by a $6^{\text {th }}$ order Fourier series.................... 212

Figure 7.7 Magnitude of acceleration due to the Moon solved by algorithm ............ 213

Figure 7.8 Sample continuous acceleration that reproduces anomaly …….................215

Figure 7.9 Numerical cost at each optimized impulse time-point............................. 216 


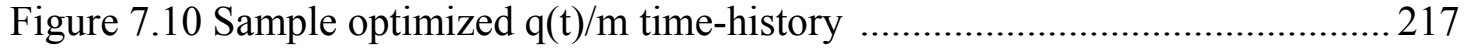

Figure 7.11 Sample optimized Lorentz accelerations in spherical coordinates ........ 217 


\section{LIST OF TABLES}

Table 2.1 Dimensionless Scale Factors …………………......................................... 15

Table 2.2 Coefficients of Drag and Lift for Geometries of Interest............................. 22

Table 2.3 Magnetization Estimates …………………………..................................... 30

Table 2.4 Dimensionless Eddy Current Scale Factors ……………………………...... 31

Table 2.5 Dimensionless Self-Capacitance Scale Factors............................................... 30

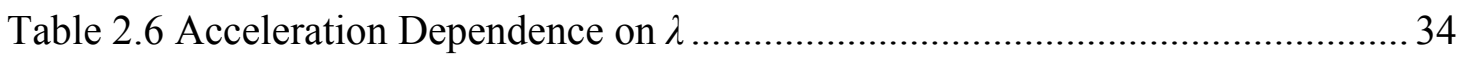

Table 2.7 Environmental Constants Used in Simulations .............................................. 35

Table 2.8 Spacecraft Constants Used in Simulations ...................................................... 36

Table 2.9 Acceleration Legend for Figures 2.7-2.18 .................................................. 37

Table 3.1 Estimated Communications Link Budget..................................................... 91

Table 3.2 Power Budget .................................................................................... 94

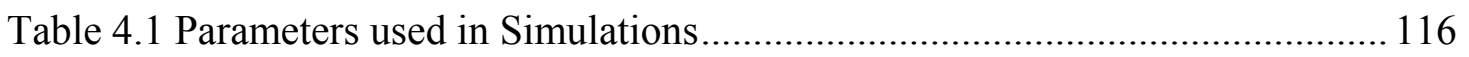

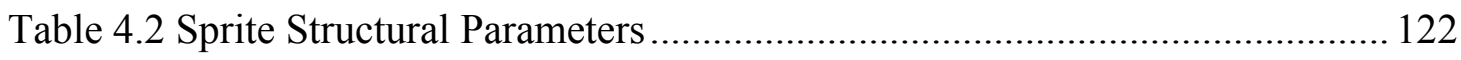

Table 4.3 Sprite Power Subsystem Parameters …………………………………..... 124

Table 4.4 Sprite Communications Subsystem Parameters .......................................... 124

Table 5.1 NASCAP Simulation Parameters ............................................................... 145

Table 5.2 NASCAP Simulation Results .................................................................. 147

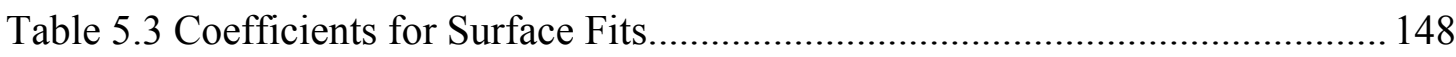

Table 5.4 Minimum Mass Budget ............................................................................ 170

Table 5.5 Selected Power and Propulsion Parameters …………………………....... 170

Table 6.1 Jovian Constants used in all Simulations ................................................... 179

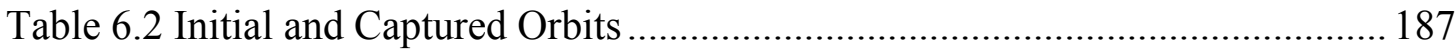

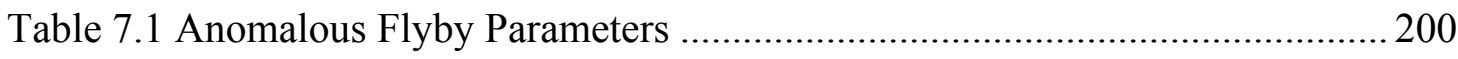

Table 7.2 Polarity and Magnitude of Anomalous State Change for NEAR................207

Table 7.3 Normalized State Errors ……………………....................................... 218 


\section{CHAPTER 1 INTRODUCTION}

This research investigates some important subtleties in natural orbital dynamicssubtleties that are typically neglected in spacecraft design. It seeks to find new equilibria and new means for transferring a spacecraft between them, all using environmental accelerations. The space environment offers a variety of wellcharacterized accelerations, but they are not typically useful for spacecraft missions because they act on extremely slow time-scales. If one could capitalize on these accelerations though, a spacecraft could be capable of propellant-free propulsion.

Eliminating the traditional dependence on propellant offers a spacecraft some important advantages. Propellantless propulsion not only enables a longer (or perhaps even unlimited) mission lifetime, it also enables qualitatively different orbits, orbits that do not obey Kepler's laws. These orbits can represent new equilibria that are not defined by gravity alone. Alternatively, these orbits can be designed to change with time in meaningful ways: adding inclination, offering new interplanetary trajectories, and introducing new synchronous solutions.

The surprising thing is that these behaviors have already been observed for decades. They occur naturally in the orbits of interplanetary dust. Interplanetary dust particles are small solid matter thought to have originated from the solar system's birth, released from comets, or ejected from planets. They range in size from a molecule to about 100 microns and can consist of a variety of materials. What makes the orbital behavior of dust particles unique is the characteristically small length scale. The same environmental forces act on dust particles as act on spacecraft, but the length scale of dust results in the forces having a much greater effect. This research is 
aimed at understanding the importance of length-scale and seeking new mission architectures that capitalize on the findings.

The approach to this task is two-fold. First, taking inspiration from the natural world, the analysis surveys what behavior has been observed by interplanetary dust and seeks to reproduce that behavior or engineer new systems based on it. This research then considers common proposals for propellantless propulsion, and seeks to understand how they might benefit from length scaling. That is, this research does not specifically offer new propellantless propulsion technologies so much as it considers current concepts in a new light.

A small number of propellant-free technologies have been proposed and tested, though none are yet commonly employed. Examples include solar sails, electrodynamic tethers, and Lorentz spacecraft. It turns out that each technology has its limitations, and these challenges have proven difficult to overcome. Though they each offer unlimited $\Delta v$ with no requirement for fuel, they come at the cost of other expenses:

- slow maneuver rates,

- indirect trajectories,

- limited payload mass,

- large or awkward spacecraft architectures,

- difficulty in ground testing, or

- challenging deployment scenarios.

These non-trivial issues have delayed or stopped any of these propellantless techniques from being utilized to date. One promising outcome of this research is an architecture that can mitigate many of these challenges.

In fact, it was in the context of considering these costs that the idea for this research was first proposed. One promising method of propellantless propulsion relies on the 
Lorentz force, an electrodynamic interaction between a moving charge and a magnetic field. This force requires that a spacecraft achieve a non-zero electrostatic charge while orbiting near a planet with a magnetic field. The effect is based on familiar principles and has been observed as a naturally occurring perturbation for orbiting dust grains in the rings of Jupiter and Saturn. Because the basic physics is well understood, the challenges are primarily technological.

The figure-of-merit for a Lorentz craft is its ratio of electrostatic charge-to-mass. This is the quantity that engineers must maximize in order to optimize the propulsive capability. Unfortunately, it is not clear how to produce arbitrarily high values of charge-to-mass on a typical spacecraft. Charge-to-mass depends on geometry, material selection, available charging mechanisms, and even the local plasma environment. Early proposals for Lorentz craft architectures involved highly charged kilometers of thin filaments extending out into space. This class of architectures is not ideal because it involves such large components and complicated deployments. So, the question arose: how do dust grains naturally achieve measurable values of chargeto-mass if it is so difficult to produce using a typical spacecraft architecture? The answer, of course, is length scale.

It turns out that the charge-to-mass ratio scales inversely with the square of the body's characteristic length. As the size of a body is reduced, its maximum charge-tomass ratio increases dramatically. So, dust grains with characteristic lengths on the order of microns naturally charge up through plasma and solar phenomena and consequently experience large accelerations associated with the Lorentz force. These accelerations can have a profound impact, even ejecting or capturing the dust into orbits at Jupiter.

This initial insight motivates the research herein, a study of length scaling in spacecraft dynamics. What specific dynamical parameters scale with length, and what 
opportunities do they afford? Can a spacecraft be designed with length scaling in mind, such that its size enables new missions? This dissertation offers eight chapters that address these questions.

Chapter 2, "A Survey of Length Scaling in Spacecraft Dynamics" is an exhaustive study and overview of the dynamical parameters that scale with length. It addresses and models orbital and rigid-body accelerations associated with gravity, relativity, radiation, particle collisions, and electromagnetic fields. The analysis focuses on order-of-magnitude trends and considers simple spacecraft shapes in orbit near Earth. The research offers metrics for comparing small spacecraft and presents length scales that small spacecraft must attain for sample missions. It identifies regimes within which the dynamics of a very small body are dominated by particular nongravitational accelerations. These regimes present opportunities for near-Earth propellantless propulsion, specifically via solar-radiation pressure, aerodynamic drag, and the Lorentz force. These results also offer guidelines for spacecraft environmental modeling, identifying which accelerations can neglected for a spacecraft of a given size in a given environment. The remaining chapters evaluate these mechanisms for a candidate spacecraft design.

Chapter 3, "A Passive, Sun-Pointing, Millimeter-Scale Solar Sail” considers a very small solid-state solar sail architecture. This analysis is the first directed at a millimeter-scale spacecraft design that, by virtue of its size, performs as a solar sail. That is, the spacecraft is propelled using photon pressure from the sun. This design represents a near-term, low-cost testbed for solar-sail demonstrations. The analysis focuses on mission opportunities for such a sail and evaluates the orbital and attitude dynamics for a passive sail in heliocentric, geocentric, and three-body orbits. Among the outcomes of this study is a passive, microfabricated attitude-control system that keeps the sail pointing towards the sun. 
Chapter 4, "Microscale Atmospheric Re-Entry Sensors" focuses on aerodynamic drag and lift as means of achieving passive ablation-free atmospheric entry, descent, and landing. This study treats the spacecraft-on-chip architecture as a wing with a high area-to-mass ratio. At very small length scales, the design is capable of both decelerating and radiating heat rapidly enough to survive and even operate throughout atmospheric entry. The analysis offers a monte-carlo simulation for the orbital and thermodynamic equations of motion that identifies ideal spacecraft-on-chip dimensions. This research has many potential applications for study of the mesospheric and space weather.

Chapters 5, 6, and 7 consider the Lorentz force. Chapter 5, “A Millimeter-Scale Lorentz-Propelled Spacecraft" gives an overview of Lorentz applications for a small spacecraft. This study identifies the trend that charge-to-mass scales with a body's characteristic length. A small spacecraft-on-chip likely represents the most feasible means for studying and testing Lorentz augmented orbits. This analysis describes a candidate architecture and mission, accounting for near-Earth plasma properties and spacecraft power requirements.

Chapter 6, "Lorentz Augmented Jovian Orbit Insertion" explores a candidate mission to Jupiter that proposes a low charge-to-mass Lorentz force orbit insertion. Jupiter's strong magnetic field and fast rate of rotation make it an ideal place to use the Lorentz force. This study assesses the required charge-to-mass ratio to capture and circularize a spacecraft's transfer orbit once it reaches Jupiter's sphere of influence. The Lorentz force architecture is then compared against a competing technology, electrodynamic tethers.

Chapter 7, "Lorentz Accelerations in the Earth Flyby Anomaly" asks whether the Lorentz force has already been experienced by traditional spacecraft in the so-called Earth Flyby Anomaly. Here six spacecraft experienced anomalous accelerations while 
executing gravity assists at Earth. Many features of the anomalous acceleration suggest that it may be associated with the Lorentz force, including that it is physical, non-conservative, bi-directional, altitude-dependent, and declination-dependent. The study focuses on the Near Earth Asteroid Rendezvous spacecraft's flyby, which demonstrated the highest anomalous change in energy with the least uncertainty. This analysis is the first of its kind to evaluate the anomaly in terms of a full six-state change, addressing the vector mechanics of the problem rather than resorting to simple scalar analyses. The study offers an algorithm for solving for the nonlinear charge-tomass time history required to reproduce the anomaly. After showing that the anomaly cannot be caused by an impulse and that continuous solutions exist, the algorithm suggests that the anomaly also cannot be fully associated with the Lorentz force. The analysis concludes that the Lorentz force can be eliminated from the list of possible sources of the anomaly.

Finally, Chapter 8 summarizes this research's contributions to the field of spacecraft dynamics and offers a discussion of open questions and future directions. The outcome of this research is a picture of length-scale as a design parameter that can enable new mission opportunities and new approaches to exploring our universe. 


\section{CHAPTER 2}

\section{A SURVEY OF LENGTH SCALING IN SPACECRAFT DYNAMICS*}

\subsection{Abstract}

Length scaling represents a new degree of freedom for spacecraft mission design. This paper presents a method for comparing the length scales of arbitrary spacecraft and uses this approach to evaluate the relevance of 12 environmental forces and torques. Three sample spacecraft geometries are considered: a sphere, a cube, and a thin square plate, at three near-Earth altitudes: $500 \mathrm{~km}, 1,000 \mathrm{~km}$, and 10,000 km. This analysis offers a guide for orbit and attitude simulations of small bodies, by suggesting which effects can and cannot be neglected for a given environment and error tolerance. This approach to length-scaling may enable extremely small spacecraft to exploit unfamiliar dynamical behaviors that result in solar-sail maneuvers, atmospheric re-entry, and Lorentz propulsion.

* Accepted with revision to the Journal of Guidance, Control, and Dynamics, March 20, 2010. Printed with permission. 


\subsection{List of Symbols}

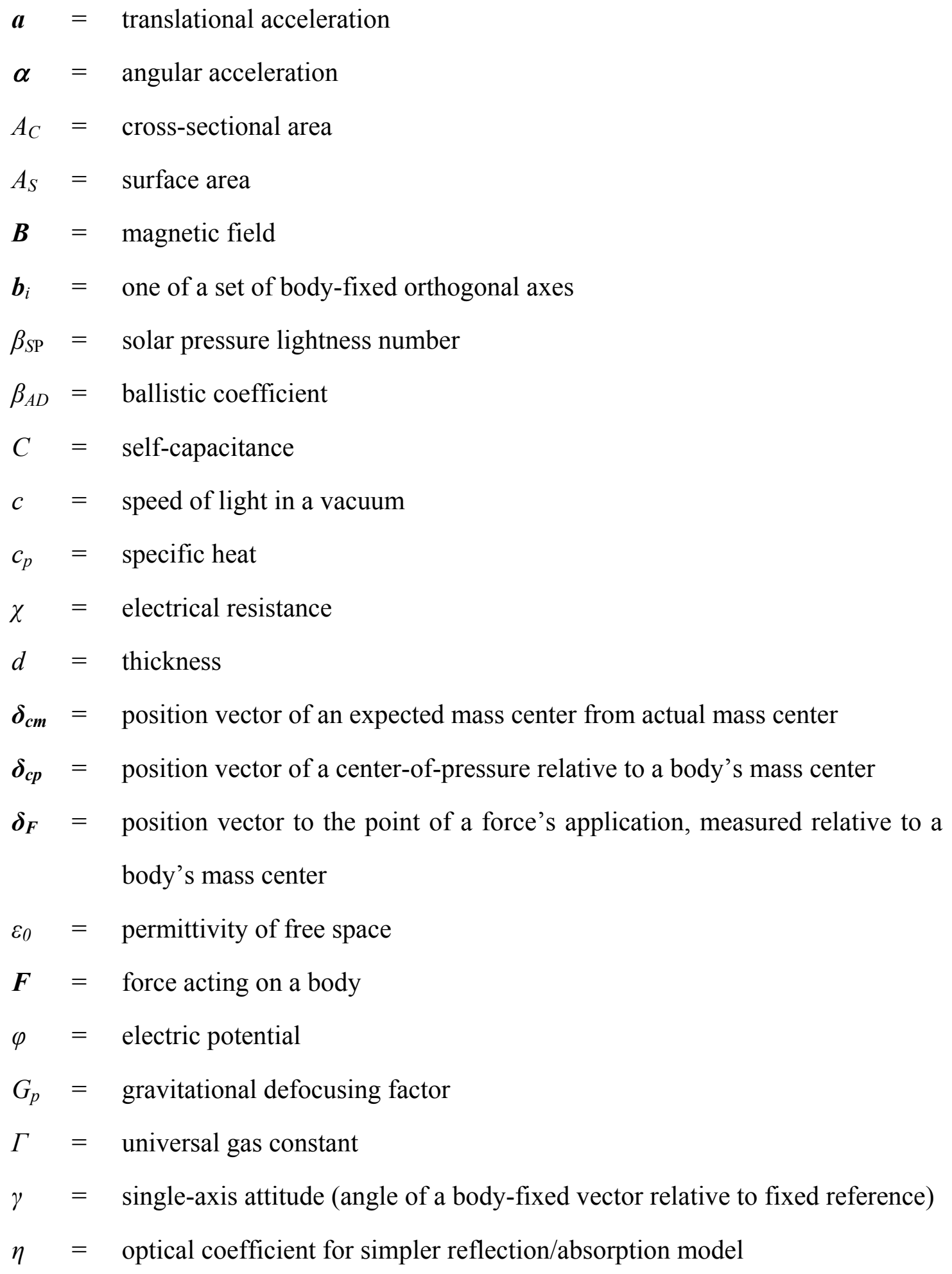




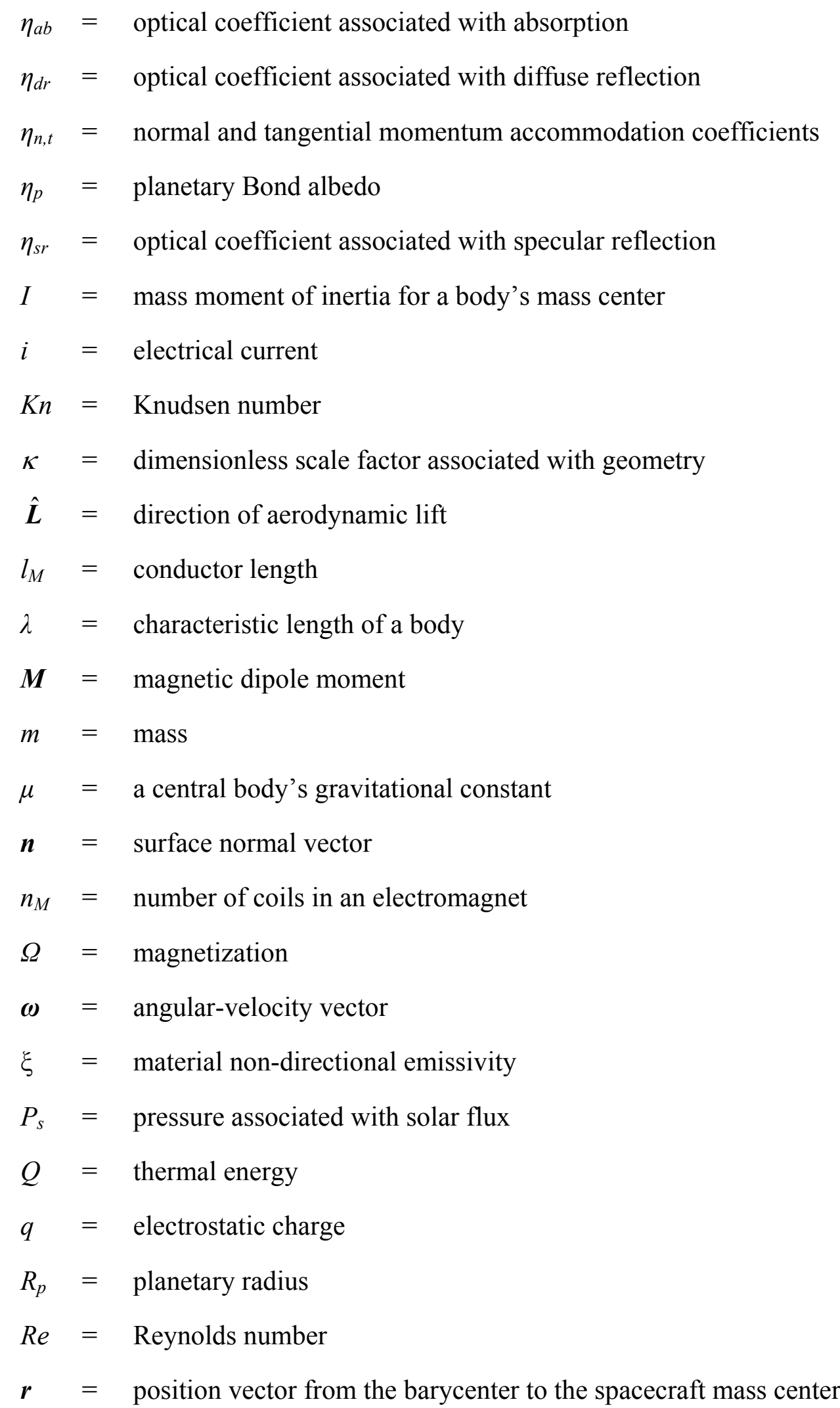




$$
\begin{aligned}
& \boldsymbol{P}=\text { pressure } \\
& \rho=\text { mass density } \\
& \rho_{A}=\text { atmosphere density } \\
& \sigma \quad=\text { material resistivity } \\
& T=\text { temperature } \\
& t \quad=\text { time } \\
& \boldsymbol{\tau}=\text { torque acting on a body } \\
& \boldsymbol{v}=\text { orbital velocity, time derivative of } \boldsymbol{r} \\
& v_{w}=\text { surface-normal velocity of molecule } \\
& W_{0}=\text { solar energy flux at distance } r_{0} \text { from the sun } \\
& \zeta \quad=\text { mean free path }
\end{aligned}
$$

\section{Subscripts}

$$
\begin{aligned}
& A D=\text { aerodynamic drag } \\
& A L=\text { aerodynamic lift } \\
& E C=\text { Eddy current } \\
& G=\text { gravity } \\
& L Z=\text { Lorentz Force } \\
& M=\text { magnetic attraction or repulsion } \\
& p=\text { planetary } \\
& P A=\text { planetary albedo } \\
& P C=\text { particle collisions } \\
& P R=\text { Poynting-Robertson drag } \\
& S P=\text { solar radiation pressure } \\
& S W=\text { solar wind } \\
& T E=\text { thermal emission }
\end{aligned}
$$




\subsection{Introduction}

Length determines the magnitude of many accelerations for which the space environment is responsible. For spacecraft designers and mission engineers, this length scaling drives common mission concerns, such as orienting a spacecraft to minimize aerodynamic drag effects or determining the magnitude of disturbance accelerations. Instead of neglecting these effects or merely accommodating them, this research seeks to exploit length scaling to achieve novel mission opportunities. The following analysis surveys most near-Earth environmental accelerations and describes a spacecraft architecture whose length scale maximizes their benefits.

This work is inspired by research in interplanetary-dust dynamics as well as advances in microfabrication techniques. Continually shrinking electronics and sensors have created a "smaller is better" paradigm. Naturally, smaller size tends to reduce weight and power, which benefits any aerospace system. While such advances enable the fabrication of an extremely small spacecraft, results from the interplanetary-dust community justify such activity. Dust particles, by virtue of a characteristic size on the order of tens of microns, experience highly non-Keplerian orbit dynamics. Solar-radiation pressure has been found to eject dust from the solar system [1,2,3], electromagnetic effects capture and eject dust in planet-centered orbits [4,5], and aerodynamic drag captures and lands dust without the bright hypersonic ablation characteristic of larger meteors [6,7]. Such effects are passive. This research is aimed at exploiting such small-body effects actively in new operations concepts.

There are other compelling reasons to develop extremely small spacecraft: economies of production, reduced launch mass, and distributed sensing opportunities. Some research has focused on developing technologies to enable a monolithic integrated-circuit (IC) silicon spacecraft design [8-15]. This architecture, sometimes 
called a "spacecraft-on-a-chip," capitalizes on advances in IC and microelectrochemical systems (MEMS) technologies. Barnhart [8] provides an historical summary of these efforts.

The present work seeks to qualify and quantify the dependence of length-scale on the orbital and angular accelerations experienced by a spacecraft in Earth orbit. A framework of scaling is introduced that considers spacecraft geometry using a set of non-dimensional coefficients. Using this framework, 12 environmental perturbations are modeled for the near-Earth space environment. This modeling follows Longuski, Todd, and König's survey of nongravitational accelerations on the Galileo spacecraft, which focuses on order-of-magnitude calculations [16]. The sources of these perturbations are associated with gravity, particle collisions, radiation, and magnetic fields. The environmental accelerations are considered for three simple and applicable geometries: a sphere, a cube, and a thin square plate. Using these test cases, the analysis relates the relative importance of each acceleration on spacecraft orbital and angular dynamics, across a range of length-scales. These results suggest sample mission applications for an IC spacecraft-on-a-chip, including solar-sailing, atmospheric reentry, and Lorentz propulsion.

\subsection{Geometric and Kinematic Scaling}

The geometry of a body of interest can be decoupled into dimensional and dimensionless scale factors. Here, units of length are generalized into a single variable $\lambda$, the characteristic length of the spacecraft. For example, the volume $V$ of a sample spacecraft is taken as the characteristic volume $\lambda^{3}$ scaled by a dimensionless factor $\kappa_{V}$

$$
V=\kappa_{V} \lambda^{3}
$$


The spacecraft's mass $m$ is the product of its volume and mean mass density $\rho$

$$
m=\kappa_{V} \rho \lambda^{3} .
$$

The density and scale factor are taken to be constant properties of the spacecraft, while $\lambda$ is an independent variable. The cross-sectional area and total surface area can be defined using $\kappa_{C}$ and $\kappa_{S}$ respectively

$$
\begin{aligned}
& A_{C}=\kappa_{C} \lambda^{2} \\
& A_{S}=\kappa_{S} \lambda^{2} .
\end{aligned}
$$

Likewise, a mass moment of inertia $I$ about the spacecraft's mass center can be treated as the product of the density and $\lambda^{5}$, scaled by an appropriate coefficient $\kappa_{I}$.

$$
I=\kappa_{I} \rho \lambda^{5}
$$

Table 1 gives the dimensionless scale factors $\kappa_{i}$ for three geometries of interest: a sphere, a cube, and a thin square plate. Figure 2.1 shows these shapes with body-fixed basis vectors $\boldsymbol{b}_{i}$. For a sphere, a shape that resembles many dust particles [2], the characteristic length is taken to be the diameter. For a cube-shaped body (e.g. the aptly named CubeSat [17]) $\lambda$ is taken to be the side length. Finally, for a thin square plate that evokes spacecraft-on-chip architectures, $\lambda$ is the length of a side. While the descriptions of the sphere and cube are one-dimensional, two parameters fully describe the square plate: the side length and the thickness. These two parameters are related by the constant aspect ratio $\kappa_{\varepsilon}$, the ratio of the thickness of the plate $d$ to the side length $\lambda$ of the square

$$
\kappa_{\varepsilon} \equiv \frac{d}{\lambda}, \kappa_{\varepsilon}<<1
$$


Previous studies $[10,11]$ have suggested an IC spacecraft-on-chip architecture with a side-length of $1 \mathrm{~cm}$ and a thickness of $25 \mu \mathrm{m}$. This size selection yields a value of $\kappa_{\varepsilon}$ $=0.0025$.

A ratio that appears in many environmental accelerations is the cross-sectional area-to-mass ratio

$$
\frac{A_{C}}{m}=\frac{\kappa_{C}}{\kappa_{V} \rho} \lambda^{-1} .
$$

The ratio depends on length-scale indirectly and geometry via $\kappa_{C} / \kappa_{V}$. For a sphere and cube, $\kappa_{C} / \kappa_{V}$ reduces to a constant value of 1.5 and 1.0 respectively. For a thin square plate, $\kappa_{C} /\left(\kappa_{V} \lambda\right)$ reduces to the plate's thickness $d=\kappa_{\epsilon} \lambda$. That is, $A_{C} / m$ is dependent only on thickness for a thin square plate geometry.

The sphere are cube have triaxial symmetry; mass moments of inertia of such a body are equal for any axis passing through the mass center. The inertia tensor $\boldsymbol{I}$ for a triaxially symmetric body can be expressed as a scalar multiple of the identity matrix

$$
\boldsymbol{I}=\kappa_{I} \rho \lambda^{5} \boldsymbol{1}_{3 \times 3} .
$$

The thin square plate has biaxial symmetry. In $\boldsymbol{b}_{\boldsymbol{i}}$ axes, which are chosen to align with the principal axes of the plate, the inertia about the mass center is

$$
\boldsymbol{I}_{\text {plate }}=\kappa_{I} \rho \lambda^{5} \operatorname{diag}\left(\left[\begin{array}{lll}
1 & 1 & 2
\end{array}\right]\right) .
$$


Table 2.1. Dimensionless Scale Factors

\begin{tabular}{cccc}
\hline \hline$\kappa_{V}$ & $\frac{\pi}{6}$ & 1 & $\begin{array}{c}\text { Thin Square } \\
\text { Plate }\end{array}$ \\
$\kappa_{I}$ & $\frac{\pi}{60}$ & $\frac{1}{6}$ & $\frac{\kappa_{\varepsilon}}{12}, \frac{\kappa_{\varepsilon}}{6}$ \\
$\kappa_{C}$ & $\frac{\pi}{4}$ & 1 & 1 \\
$\kappa_{S}$ & $\pi$ & 6 & 2 \\
\hline \hline
\end{tabular}
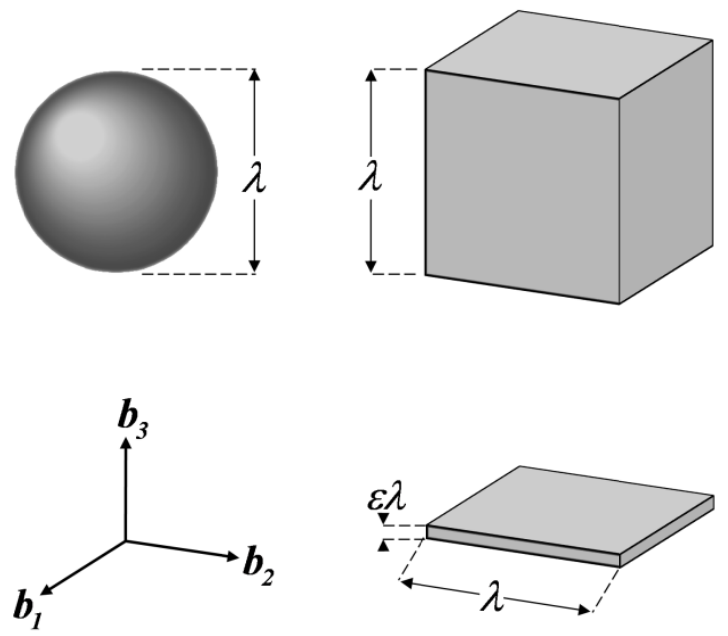

Figure 2.1. Characteristic length and axis of rotation for geometries of interest: a.) sphere, b.) cube, and c.) thin plate.

Scaling also appears in the orbital and attitude equations of motion. Newton's second law, written in terms of acceleration, includes an implicit dependence on $\lambda$

$$
a=\frac{F}{m}=\frac{F}{\kappa_{V} \rho} \lambda^{-3}
$$

Likewise $\lambda$ appears in angular accelerations. If a force acts at a point $\boldsymbol{\delta}_{\boldsymbol{F}}$ measured from the spacecraft's mass center, a torque $\boldsymbol{\tau}$ results 


$$
\begin{gathered}
\boldsymbol{\tau}=\boldsymbol{\delta}_{F} \times \boldsymbol{F}, \\
\tau=\kappa_{T} F \lambda .
\end{gathered}
$$

Here, the scale factor $\kappa_{T}$ is defined to account for both the location and orientation of the force's application. Angular acceleration is related to torque according to Euler's equation of motion for a rigid body [18]

$$
\boldsymbol{I} \cdot \dot{\boldsymbol{\omega}}+\boldsymbol{\omega} \times \boldsymbol{I} \cdot \boldsymbol{\omega}=\boldsymbol{\tau}
$$

For triaxially symmetric geometries, as in the sphere and cube, $\boldsymbol{\omega} \times \boldsymbol{I} \cdot \boldsymbol{\omega}$ reduces to $\boldsymbol{0}$. Alternatively, for non-symmetric geometries, one can consider the case of slow spin, $\mathrm{I} \omega^{2} \ll \tau$, with nutation damping. These cases simplify the analysis, such that angular acceleration $\alpha \equiv \dot{\omega}$ is proportional to $I$

$$
\alpha=\frac{\tau}{I}=\frac{\kappa_{T} F}{\kappa_{I} \rho} \lambda^{-4}
$$

$\alpha$ can be thought of as the ratio of torque to inertia, or roughly as the angular acceleration. With this framework of dimensionless constants, a spacecraft's sensitivity to characteristic length can be explored in terms of each environmental force and torque model.

\subsection{Gravity}

The following analysis verifies that gravitational accelerations are uniquely lengthindependent and suggests that the opportunities and challenges associated with gravity gradient torques, n-body maneuvers, and planetary oblateness effects are present at any length scale. 


\subsubsection{Two-Body Orbits}

Newton's Law of Universal Gravitation gives the force acting between two bodies

$$
\boldsymbol{F}_{G}=-\frac{\mu}{r^{2}} m \hat{\boldsymbol{r}}
$$

where the substitution $\mu=G m_{p}$ is the product of the universal gravitational constant $G$ and the opposing body's mass $m_{p}$. The magnitude of this force scales with the spacecraft's mass and thus with $\lambda^{3}$

$$
F_{G}=\kappa_{V} \rho \frac{\mu}{r^{2}} \lambda^{3}
$$

The acceleration is the familiar, length-independent right-hand side of the two-body equation of motion

$$
\boldsymbol{a}_{G}=-\frac{\mu}{r^{2}} \hat{\boldsymbol{r}}
$$

As long as $m \ll m_{p}$, the characteristic size of the orbiting body is unimportant.

Gravitational force is said to act at a body's center of gravity, a point that can differ from a body's center-of-mass because gravity depends on distance from the central body. As a result, the force due to gravity can apply the familiar gravitygradient torque. This torque is related to the body's inertia tensor and the direction of the gravitational acceleration $-\hat{\boldsymbol{r}}[19]$

$$
\boldsymbol{\tau}_{G}=\frac{3 \mu}{r^{3}}(\hat{\boldsymbol{r}} \times \boldsymbol{I} \cdot \hat{\boldsymbol{r}})
$$

For a sphere or cube, triaxial symmetry reduces the vector product $\hat{\boldsymbol{r}} \times \boldsymbol{I} \cdot \hat{\boldsymbol{r}}$ to $\boldsymbol{0}$ for any attitude, indicating that there is no applied torque. For the thin square plate, rotation about any axis perpendicular to $\boldsymbol{b}_{3}$, as illustrated in Figure 2.2, reduces this 
term to $\|\hat{\boldsymbol{r}} \times \boldsymbol{I} \cdot \hat{\boldsymbol{r}}\|=I_{1} \cos \gamma_{G} \sin \gamma_{G}$ where $\cos \gamma_{G}=\hat{\boldsymbol{r}} \cdot \boldsymbol{b}_{3}$. The zero-torque equilibria are at $\gamma_{G}=k \frac{\pi}{2}$, where $k$ is an integer. Odd values of $k$ represent marginally stable equilibria; even values of $k$ represent unstable equilibria. More simply, gravity gradient torques tend to orient a square plate such that $\hat{\boldsymbol{r}} \perp \boldsymbol{b}_{3}$.

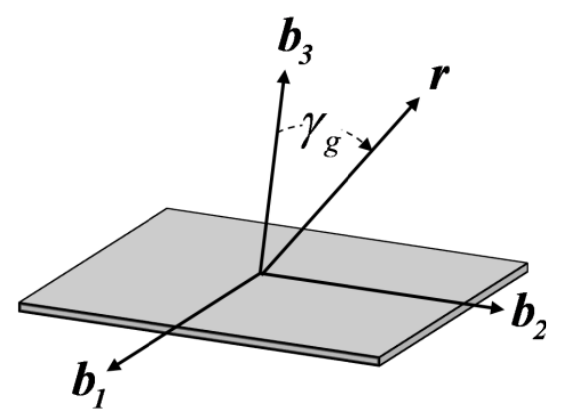

Figure 2.2. Angle of rotation associated with gravity gradient torques.

A mass imbalance can introduce unexpected gravity-gradient torque. For a deviation $\boldsymbol{\delta}_{c m}$ of the mass from the expected mass center, the parallel axis theorem gives the inertia $\boldsymbol{I}_{\boldsymbol{\delta}}$ about the shifted mass-center [18]

$$
\boldsymbol{I}_{\boldsymbol{\delta}}=\boldsymbol{I}+m\left(\delta_{c m}{ }^{2} \boldsymbol{1}_{3 \times 3}-\boldsymbol{\delta}_{c m} \boldsymbol{\delta}_{c m}\right)
$$

This results in a gravity gradient torque proportional to $\left(m \hat{\boldsymbol{r}} \times \boldsymbol{\delta}_{c m} \boldsymbol{\delta}_{c m} \cdot \hat{\boldsymbol{r}}\right)$, a term that goes to zero as $\boldsymbol{\delta}_{c m} \| \hat{\boldsymbol{r}}$. For a plate geometry with a mass-center displacement in the $\boldsymbol{b}_{1}-\boldsymbol{b}_{2}$ plane, the magnitude of the resultant torque goes with $\kappa_{V} \lambda^{3} \delta_{c m}{ }^{2} \cos \gamma_{G} \sin \gamma_{G}$ with equilibria at $\hat{\boldsymbol{r}} \perp \boldsymbol{b}_{3}$ and $\boldsymbol{\delta}_{c m} \| \hat{\boldsymbol{r}}$.

\subsubsection{Higher Order Gravitational Accelerations}

Gravity is also responsible effects such as accelerations associated with secondary bodies [20], central-body non-spherical mass distribution [19], general relativity [21], 
Lense-Thirring frame-dragging [21,22], and ocean and planetary tides [23]. In each of these cases, the accelerations are still length-independent. Any maneuver based on these physics, such as a low-energy transfer orbit, is equally possible for a small or large spacecraft.

\subsection{Particle Collisions}

Spacecraft experience accelerations due to collisions with very small particles. These particles are associated with atmospheres, interplanetary dust, and solar wind. The accelerations that these collisions produce scale with $A_{C} / m$, implying that the associated acceleration depends on $\lambda^{-1}$.

$$
a \propto \frac{A_{C}}{m} \propto \frac{1}{\lambda} .
$$

A smaller body is more sensitive than a large body to particle-collision accelerations.

Given a spacecraft that is much larger than the mean distance between particles, collision forces can be modeled as a pressure that acts at an effective center of pressure $\boldsymbol{\delta}_{\boldsymbol{C} \boldsymbol{P}}$. For the regular shapes considered here, this force acts through the center of mass. Therefore, these three shapes experience torque-free particle collisions. In practice, an arbitrarily small dislocation of $\boldsymbol{\delta}_{C P}$ produces a torque of the form in Eq.

2.10. This torque scales with $\lambda^{3}$, resulting in an angular acceleration that scales with $\lambda^{-2}$.

$$
\alpha \propto \frac{A_{C} \kappa_{\tau} \lambda}{I} \propto \lambda^{-2} .
$$

If the spacecraft is sufficiently small (e.g. at the length-scale of the particles themselves), particle collisions are more rare and can no longer accurately be modeled as a pressure. 


\subsubsection{Aerodynamic Forces}

Bodies traveling through an atmosphere experience aerodynamic forces. In the rarified upper atmosphere that Low Earth Orbits (LEO) experience, aerodynamic drag often dominates the non-gravitational accelerations. This drag acts in the direction opposite velocity, removing kinetic energy and angular momentum from the orbit [23]. Here, drag is evaluated in a model with simplified, hyperthermal, free-molecular flow that neglects spinning and tumbling body effects. The force's magnitude is proportional to the body's cross-sectional area, a quantity that scales with $\lambda^{2}$ due to area dependence [23]

$$
\boldsymbol{F}_{A \boldsymbol{D}}=-\frac{1}{2} \kappa_{A D} A_{C} \rho_{A} v^{2} \hat{\boldsymbol{v}}
$$

The local atmospheric density is given by $\rho_{a}$, a quantity that encapsulates the force's strong dependence on altitude and solar activity. Some shapes also experience aerodynamic lift, which takes a form similar to that of aerodynamic drag [23]

$$
\boldsymbol{F}_{A \boldsymbol{L}}=\frac{1}{2} \kappa_{A L} A_{C} \rho_{A} v^{2} \hat{\boldsymbol{L}}
$$

This force is directed along $\hat{\boldsymbol{L}}$, a vector perpendicular to $\hat{\boldsymbol{v}}$ and in the plane of $\boldsymbol{F}_{\boldsymbol{A D}}$ and $\boldsymbol{b}_{3}$, as illustrated in Figure 2.3.

The coefficients of drag $\kappa_{A D}$ and lift $\kappa_{A L}$ account for the surface interactions associated with diffuse and specular reflection, as well as molecular accommodation, the proportion of momentum imparted by the impacting molecules [26]. Table 2.2 gives characteristic values these coefficients. A flat plate behaves like an airfoil in that it has coefficients that vary with attitude. Here, the so-called angle of attack is referenced to $\boldsymbol{b}_{3}, \cos \gamma_{A}=\boldsymbol{b}_{3} \cdot \hat{\boldsymbol{v}}$. Storch gives equations for $\kappa_{A D}$ and $\kappa_{A L}$ for a flat plate in hyperthermal free-molecular flow. These equations, in terms of $\gamma_{A}$ are [26] 


$$
\begin{gathered}
\kappa_{A D}=2\left[\eta_{t}+\eta_{n} \frac{v_{w}}{v} \cos \left(\gamma_{A}\right)+\left(2-\eta_{n}-\eta_{t}\right) \cos ^{2}\left(\gamma_{A}\right)\right] \cos \left(\gamma_{A}\right) . \\
\kappa_{A L}=\left[\eta_{n} \frac{v_{w}}{v}+\left(2-\eta_{n}-\eta_{t}\right) \cos \left(\gamma_{A}\right)\right] \sin \left(2 \gamma_{A}\right),
\end{gathered}
$$

Molecular accommodation is modeled with the normal and tangential momentumaccommodation coefficients $\eta_{n}$ and $\eta_{t}$ respectively. They are a function of both the surface and fluid properties. The normal component of the thermal velocity of molecules escaping the plate's surface $v_{w}$ is given by [26]

$$
v_{w}=\sqrt{\frac{\pi \Gamma T}{2}},
$$

where $\Gamma$ is the specific gas constant and $T$ is the surface temperature of the plate. Setting $T$ to the local atmospheric temperature and using circular LEO velocities yields characteristic values for $v_{w} / v$ of roughly 0.05 . Figure 2.4 plots Eq. 2.24 and 2.23 over angle-of-attack for these sample conditions. Positive lift is associated with $\cos \left(\hat{\boldsymbol{F}}_{A L} \cdot \hat{\boldsymbol{r}}\right)>0$. Both coefficients go to zero at $\hat{\boldsymbol{v}} \perp \boldsymbol{b}_{3}$, the equilibrium attitude. At $\hat{\boldsymbol{v}} \perp \boldsymbol{b}_{3}$ there is no lift, and drag is maximized, especially since the full square area is leading.

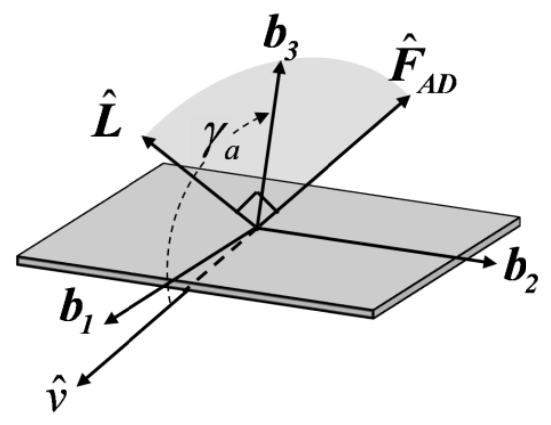

Figure 2.3. Orientation of lift and drag forces on a flat plate in a fluid flow. 
Table 2.2. Coefficients of Drag and Lift for Geometries of Interest

\begin{tabular}{cccc}
\hline & $\begin{array}{c}\text { Sphere } \\
{[24,25]}\end{array}$ & $\begin{array}{c}\text { Cube } \\
{[24,25]}\end{array}$ & $\begin{array}{c}\text { Thin Square } \\
\text { Plate [26] }\end{array}$ \\
\hline$\kappa_{A D}$ & 2.2 & 2.2 & Eq 2.23 \\
$\kappa_{A L}$ & 0 & 0 & Eq 2.24 \\
\hline \hline
\end{tabular}

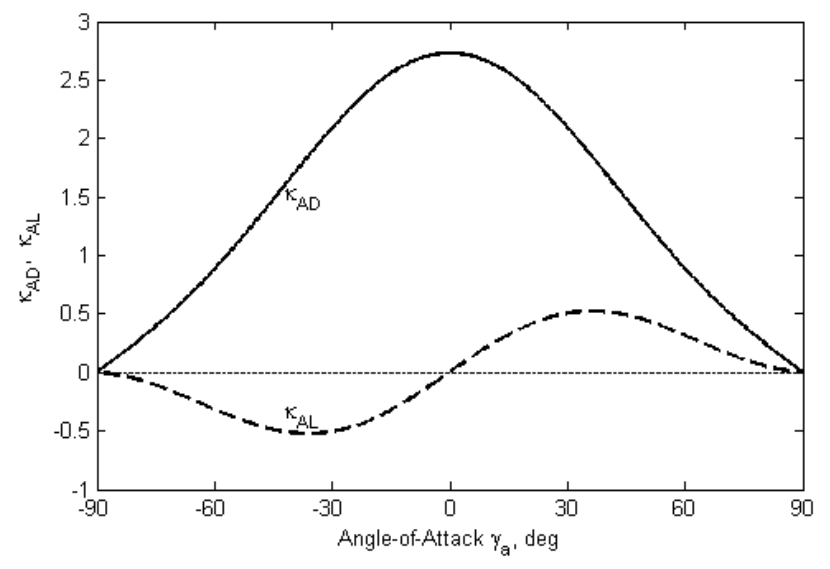

Figure 2.4. Drag and lift coefficients vs. angle-of-attack for a flat plate in hyperthermal free-molecular flow with $\eta_{n}=\eta_{t}=0.7$ and $v_{w} / v=0.05$ [26].

Aerodynamic accelerations scale with the critical ratio of $A_{C} / m$

$$
a_{A D}=\frac{1}{2} \kappa_{A D} \frac{A_{C}}{m} \rho_{A} v^{2}=\frac{\kappa_{A D} \kappa_{C}}{2 \kappa_{V} \rho} \rho_{A} v^{2} \lambda^{-1} .
$$

\subsubsection{Micrometeoroid Collisions}

The solar system is populated by small dust and meteoroid particles orbiting the sun and planets. As these particles collide with a body, they impart momentum, 
similar to the mechanism of aerodynamic forces. The force associated with these impacts can be modeled by [16]

$$
\boldsymbol{F}_{\boldsymbol{P}}=\dot{m}_{P} A_{C} \boldsymbol{v}_{\boldsymbol{P C}},
$$

where $\dot{m}_{P}$ is the mass flux rate of particles with velocity $\boldsymbol{v}_{\boldsymbol{P C}}$ relative to the impacted body. Near Earth, the mean particle velocity is roughly directed Earthward

$$
\boldsymbol{v}_{\boldsymbol{P C}}=G_{P} \times 20\left[\frac{k m}{s}\right]\left(-\hat{\boldsymbol{r}}_{\boldsymbol{E}}\right),
$$

and is a function of the gravitational defocusing factor $G_{P}[27,19]$

$$
G_{P}=0.57+0.43 \frac{R_{E}}{r_{E}}
$$

where $R_{E}$ is the radius of the Earth.

\subsubsection{Solar Wind}

The sun ejects ionized protons and electrons from its upper atmosphere. Like micrometeoroids, these particles impart energy and momentum when they impact a body. This interaction can be modeled with an effective momentum flux $\dot{p}_{S W}$ that varies with the solar distance $r_{s}^{2}[16]$

$$
\boldsymbol{F}_{S W}=\dot{p}_{S W}\left(\frac{r_{0}}{r_{s}}\right)^{2} A_{c} \hat{\boldsymbol{r}}_{s},
$$

where $r_{0}$ is a reference distance. Most are rejected by Earth's magnetopause, so $\dot{p}_{S W}$ can be thought to represent an upper-limit in the near-Earth environment [28]. 


\subsection{Radiation}

Photons have both energy and momentum, which both are related to wavelength. When a photon is absorbed or reflected, momentum is exchanged. The sources of photons considered here include solar radiation, planetary albedo reflection, and thermal radiation. These can be modeled as pressures, whose resultant force is a function of the exposed area $A_{c}$ and the surface characteristics, which determine how the incoming photons are specularly reflected, diffusely reflected, or absorbed. The dimensionless fractions $\eta_{s r}, \eta_{d r}$, and $\eta_{a b}$ account for each of these respective effects for a given wavelength, $\eta_{s r}+\eta_{d r}+\eta_{a b}=1$. Accelerations associated with radiation scale with the familiar ratio $A_{c} / m$.

For a radiation pressure $\boldsymbol{P}$, the force acting on a sphere is [19]

$$
\boldsymbol{F}=A_{C}\left[1+\frac{4}{9} \eta_{d r}\right] \boldsymbol{P}
$$

and for a flat surface with normal $\hat{\boldsymbol{n}}$ as illustrated in Figure 2.5 [19]

$$
\boldsymbol{F}_{S}=P A_{C}(\hat{\boldsymbol{P}} \cdot \hat{\boldsymbol{n}})\left[\left(2 \eta_{s r}(\hat{\boldsymbol{P}} \cdot \hat{\boldsymbol{n}})+\frac{2}{3} \eta_{d r}\right) \hat{\boldsymbol{n}}+\left(\eta_{a b}+\eta_{d r}\right) \hat{\boldsymbol{P}}\right] .
$$

For the sake of added intuition, this analysis assumes that a cube experiences radiation forces similar to those that a sphere experiences (given Eq 2.31), and adopts a simple specular reflection model for the flat plate, taking $\cos \gamma_{s}=(\hat{\boldsymbol{P}} \cdot \hat{\boldsymbol{n}})$ as the pitch angle [29]

$$
\boldsymbol{F}_{S}=2 \eta_{S} P A_{C} \cos ^{2} \gamma_{s} \hat{\boldsymbol{n}}
$$

Here, the surface properties are captured in the single coefficient $\eta_{S}$. The direction of these accelerations acting on a plate is a function of the orientation of the surfacenormal vector. Thus the orbital and attitude dynamics can be highly coupled. The 
force acts at an effective center of pressure. As before, any non-zero $\delta_{C P}$ produces a torque according to Eq. 2.10, resulting in an angular acceleration that scales with $\lambda^{-2}$.

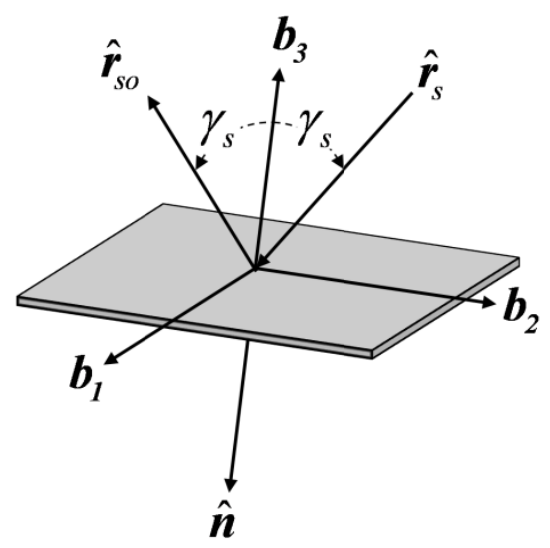

Figure 2.5. Pitch angle and relevant vectors associated with solar radiation pressure.

\subsubsection{Solar Radiation Pressure}

Photon flux originating from the sun produces an effective pressure $\boldsymbol{P}_{\boldsymbol{S R} \boldsymbol{P}}$ that can be modeled by [29]

$$
\boldsymbol{P}_{S R P}=\frac{W_{0}}{c}\left(\frac{r_{0}}{r_{s}}\right)^{2} \hat{\boldsymbol{r}}_{s},
$$

where $c$ is the speed of light, $W_{0}$ is the energy flux from the sun taken at a reference distance $r_{0}$.

\subsubsection{Planetary Albedo}

Solar flux is also reflected from planets or moons in the solar system. A body orbiting a reflective planet experiences these photons as a secondary pressure 
originating from the planet's center. Blanco and McCuskey give a model that estimates a maximum radiation pressure from a diffusely reflecting planet at a distance $r_{p}[30]$

$$
\boldsymbol{P}_{\boldsymbol{P A}}=P_{s}\left(\frac{2}{3} \eta_{p} \frac{R_{p}{ }^{2}}{r_{p}{ }^{2}}\right) \hat{\boldsymbol{r}}_{\boldsymbol{p}},
$$

where $R_{p}$ is the planet's radius and and $\eta_{p}$ is the planet's Bond albedo, the fraction of incident solar power that is reflected.

\subsubsection{Thermal Emission}

Energy and momentum are also associated with thermal emission. The StephanBoltzmann law gives the thermal power emitted from an area surface element at a temperature $T$ with emissivity $\xi$

$$
\frac{d E}{d t}=\sigma \xi T^{4} d A
$$

The force associated with this thermal radiation is then [31]

$$
\boldsymbol{d} \boldsymbol{F}_{T E}=\frac{\sigma \xi T^{4}}{c} \boldsymbol{d A}
$$

Taking the surface to be isothermal, one can integrate Eq. 2.37 and find that regular shapes experience no net force or torque. However, if the temperature is non-uniform, a net force results opposite the direction of the temperature gradient. The temperature gradient and consequent force for a spinning body are known as the Yarkovsky force [2], an effect neglected in the present analysis. 


\subsubsection{Planetary Thermal Emission}

Secondary bodies near an emitter can intercept the radiated power and experience a pressure. For a planet with radius $R_{p}$, temperature $T_{p}$, and emissivity $\xi_{p}$, the effective pressure goes with distance $r_{p}$ squared [16]

$$
\boldsymbol{P}_{\boldsymbol{T E}}=\frac{\sigma \xi_{p} T_{p}^{4}}{c}\left(\frac{R_{p}}{r_{p}}\right)^{2} \hat{\boldsymbol{r}}_{\boldsymbol{p}} .
$$

\subsubsection{Poynting-Robertson Drag}

If thermal radiation is anisotropic in an inertial frame, a secondary effect known as Poynting-Robertson drag results. This effect is associated with the motion of a radiating body. Here, the difference in the Doppler shifts between the thermal energy radiated in the velocity and anti-velocity directions produces a force [32]. As a hot body orbits, heat radiated forward (along $\hat{\boldsymbol{v}}$ ) is blueshifted by the orbital velocity, implying a higher energy level than the radiation associated with a static body. Alternatively, heat that is radiated backwards is redshifted by the orbital velocity. The result of this effect is a force acting in the drag direction $(-\hat{\boldsymbol{v}})$ that removes energy from the orbit. This drag is given by [23]

$$
\boldsymbol{F}_{P R}=-\frac{\sigma \xi T^{4}}{c^{2}} A_{C} \boldsymbol{v}
$$

a quantity that is generally small owing to the $c^{-2}$ term. 


\section{$2.8 \quad$ Magnetic Fields}

Magnetic fields in the space environment can affect a spacecraft's orbit and attitude through a variety of mechanisms.

\subsubsection{Magnetic Attraction and Repulsion}

Magnetic fields are commonly considered in the design of spacecraft attitude subsystems as either actuators (e.g. torque rods) or disturbances. However, magnetic fields can be produced on a spacecraft both intentionally and unintentionally. In both cases, a dipole-field model is generally sufficient for evaluating the force and torque.

The interaction between a spacecraft-fixed and local environmental magnetic field produces a force according to [33]

$$
\boldsymbol{F}_{\boldsymbol{M}}=\nabla\left(\boldsymbol{M}_{s c} \cdot \boldsymbol{B}\right)
$$

where $\boldsymbol{M}_{\boldsymbol{s c}}$ is the magnetic moment of the spacecraft and $\boldsymbol{B}$ is the local environmental magnetic field. For the case of a dipole approximation of the spacecraft and planetary magnetic fields (given dipole moment $\boldsymbol{M}_{\boldsymbol{P}}$ ), this equation can be approximated by [34]

$$
\boldsymbol{F}_{\boldsymbol{M}}=\frac{3 \mu_{0}}{4 \pi r_{M}{ }^{4}}\left(\begin{array}{c}
\left(\hat{\boldsymbol{r}}_{\boldsymbol{M}} \times \boldsymbol{M}\right) \times \boldsymbol{M}_{\boldsymbol{P}}+\left(\hat{\boldsymbol{r}}_{\boldsymbol{M}} \times \boldsymbol{M}_{\boldsymbol{P}}\right) \times \boldsymbol{M}-2 \hat{\boldsymbol{r}}_{\boldsymbol{M}}\left(\boldsymbol{M} \cdot \boldsymbol{M}_{\boldsymbol{P}}\right) \\
+5 \hat{\boldsymbol{r}}_{\boldsymbol{M}}\left(\left(\hat{\boldsymbol{r}}_{\boldsymbol{M}} \times \boldsymbol{M}\right) \cdot\left(\hat{\boldsymbol{r}}_{\boldsymbol{M}} \times \boldsymbol{M}_{\boldsymbol{P}}\right)\right)
\end{array}\right)
$$

where $\mu_{0}$ is the permeability of free space and $\boldsymbol{r}_{\boldsymbol{M}}$ is the vector separating the planet and spacecraft centers. Experience suggests that this force tends to be negligibly small, even for powerful magnets located at the Earth's surface. However, the torque applied between the two magnetic fields can be significant [19]

$$
\tau_{M}=M_{s c} \times B .
$$


This "compass torque" is the magnetic effect that is most often an issue for spacecraft.

For a permanent magnet, the magnetic moment is a function of the geometry and magnetization $\boldsymbol{\Omega}$, the dipole moment strength per unit volume, roughly [35]

$$
\boldsymbol{M}_{s c}=\kappa_{V} \lambda^{3} \boldsymbol{\Omega} .
$$

The direction of magnetization points from the magnet's south pole to north pole. The magnetic dipole moment of a permanent magnet goes with $\lambda^{3}$. As a consequence, angular acceleration due to magnetic torques is proportional to $\lambda^{-2}$.

For an electromagnet with $n_{M}$ coils of conductor with current $i$, the dipole moment is given by [39]

$$
\boldsymbol{M}_{s c}=\oint i d A=n_{M} i A
$$

where $A$ is the area effectively enclosed by each coil, e.g. $A_{C}$. Electrical resistance $\chi$ is the product of the conductor's material resistivity $\sigma_{M}$ and the ratio of the conductor's length $l_{M}$ to its cross-sectional area $A_{M}[35]$

$$
\chi=\sigma_{M} \frac{l_{M}}{A_{M}} .
$$

As the current or number of coils increases, a larger conductor cross section is required to maintain the same $i^{2} \chi$ power losses. Consequently, the dipole magnetic moment of an electromagnet goes also with $\lambda^{3}$ [35]. The magnetization model in Eq. 2.43 can therefore be applied to both permanent magnets and current-carrying coils. Table 2.3 gives an estimate for the magnetization of three cases: a rare Earth permanent magnet, a non-spinning spacecraft with an unintentional residual magnetic field, and commercially available magnetic torquers. According to this model, magnetic orbital acceleration is length-independent, and angular acceleration scales with $\lambda^{-2}$. 
Table 2.3. Magnetization Estimates

\begin{tabular}{ccc} 
Source & $\Omega, \mathrm{A} / \mathrm{m}$ & Ref. \\
\hline Rare Earth Permanent Magnets & $8 \times 10^{5}$ & {$[36]$} \\
$\Omega_{R}$, Unintentional Residual & $0.1-1.0$ & {$[37]$} \\
Spacecraft Magnetization & & \\
$\Omega_{T}$, Magnetic Torquers & $200-6400$ & {$[38]$} \\
\hline \hline
\end{tabular}

\subsubsection{Eddy Current Damping}

A conductive body moving through a magnetic field experiences a damping effect associated with eddy currents. The changing magnetic field within the conductor drives electrons, which set up current loops. Resistance in the material dissipates these currents as heat, removing energy from the system. The net effect is a force and torque opposite the direction of motion.

Accurately modeling eddy currents is challenging [39]. As in the case of magnetic attractive and repulsive forces, the magnitude of the eddy current force is exceedingly small. Eddy current torque, however, can significantly affect spacecraft attitude [37]

$$
\boldsymbol{\tau}_{E C}=-\varepsilon_{E C} \boldsymbol{B} \times(\boldsymbol{\omega} \times \boldsymbol{B}) .
$$

The torque is a source of damping because it acts opposite and proportional to angular velocity. The constant $\varepsilon_{E C}$ is associated with the body's geometry and resistivity [37]

$$
\varepsilon_{E C}=\frac{\kappa_{E C}}{\sigma} \lambda^{5}
$$

Expressions for $\varepsilon_{E C}$ have been calculated for thin-shelled spheres and cylinders, as well as a circular loop of wire. Here, the sphere and cube are both treated as thinshelled spheres with thickness $\kappa_{\varepsilon} \lambda$, while the plate is treated as a circle of wire. The 
corresponding coefficients $\kappa_{\mathrm{EC}}$ are given in Table 2.4. Since torque is proportional to $\lambda^{5}$, angular acceleration is length-independent [40].

Table 2.4. Dimensionless Eddy Current Scale Factors

\begin{tabular}{cccc} 
& $\begin{array}{c}\text { Sphere } \\
{[40]}\end{array}$ & $\begin{array}{c}\text { Cube } \\
{[40]}\end{array}$ & Thin Square Plate [37] \\
\hline$\kappa_{E C}$ & $\frac{\pi}{24} \kappa_{\varepsilon}$ & $\frac{\pi}{24} \kappa_{\varepsilon}$ & $\frac{\pi}{32} \kappa_{C S}$ \\
\hline
\end{tabular}

\subsubsection{The Lorentz Force}

A charged body traveling through a rotating magnetic field experiences the Lorentz force. Here, electrostatic charge can transfer orbital energy and momentum to and from a planet through its co-rotating magnetic field via the Lorentz force. The Lorentz force $\boldsymbol{F}_{\boldsymbol{L} Z}$ acting on an orbiting body with electrostatic charge $q$ is $[41,42]$

$$
F_{L Z}=q v_{B} \times B
$$

where $\boldsymbol{v}_{\boldsymbol{B}}$ is the velocity relative to a magnetic field $\boldsymbol{B}$. For a magnetic field rotating with an angular velocity $\boldsymbol{\omega}_{B}$ the relative velocity is [42]

$$
v_{B}=v-\omega_{B} \times r_{B}
$$

where $\boldsymbol{r}_{\boldsymbol{B}}$ is the body's position with respect to the magnetic field's center, usually taken to be a planet's center-of-mass. The direction of this force is dictated by the body's orbit and the local magnetic field; $q$ can only modulate the force's magnitude along this direction. Figure 2.6 illustrates a sample equatorial, retrograde, elliptical orbit around Earth. The arrows indicate the direction and relative magnitude of the Lorentz force at various positions for a positively charged body. The force is largest 
at perigee, where the body's velocity and the local magnetic field are both maximized. The force can do work on the body's orbit at positions where $\boldsymbol{F}_{\boldsymbol{L} \boldsymbol{Z}}$ has a component along $\hat{\boldsymbol{v}}$.

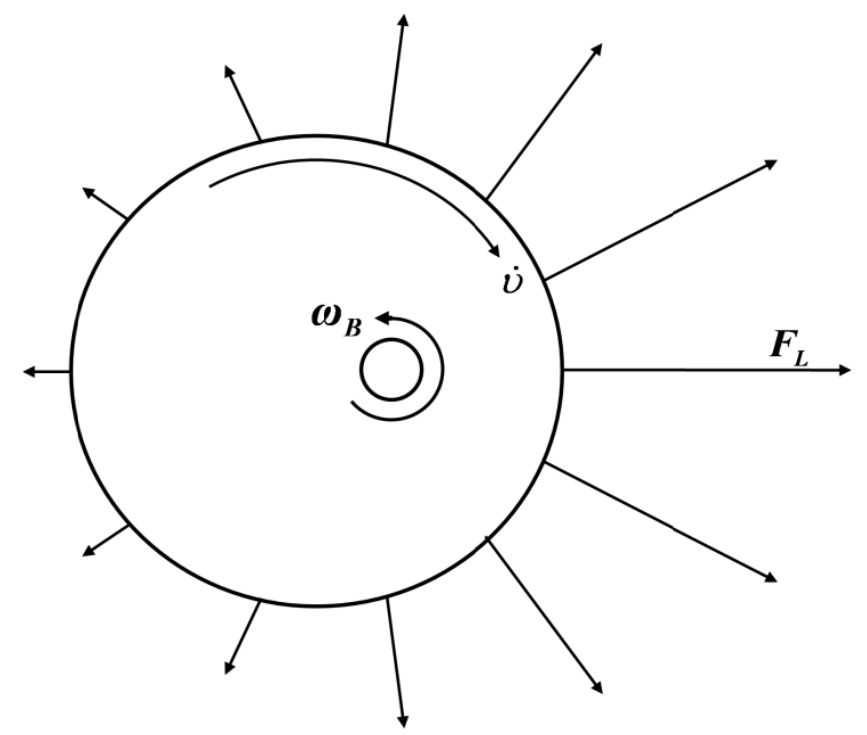

Figure 2.6. Direction of Lorentz force throughout a retrograde elliptical Earth orbit for $q>0$.

The associated Lorentz acceleration scales with $q / m$, the charge-to-mass ratio

$$
\boldsymbol{a}_{L Z}=\frac{q}{m} \boldsymbol{v}_{\boldsymbol{r}} \times \boldsymbol{B}
$$

This ratio is a function of the body's geometry, electrostatic potential, and the local space plasma environment. For a desired charge to be established, a biased electric potential $\phi$ must be generated on a body with sufficiently large self-capacitance $C$

$$
q=C \phi
$$

In a vacuum, self-capacitance is a function of geometry only. The three geometries of interest have capacitance expressions that take the form $[43,44]$ 


$$
C=\kappa_{C A P} 4 \pi \varepsilon_{0} \lambda
$$

where $\varepsilon_{0}$ is the permittivity of free space and $\kappa_{\mathrm{CAP}}$ is a dimensionless coefficient given in Table 2.5 for each shape. These models suggest that $q / m$ and the consequent Lorentz acceleration scales with $\lambda^{-2}$

$$
\frac{q}{m}=\frac{4 \pi \varepsilon_{0} \kappa_{C A P}}{\kappa_{V} \rho} \phi \lambda^{-2}
$$

The environment around a planetary magnetic field generally consists of rarefied plasma whose presence increases the effective self-capacitance. As a result, these values can be treated as lower bounds.

Table 2.5. Dimensionless Self-Capacitance Scale Factors

\begin{tabular}{cccc}
\hline Sphere & $\begin{array}{c}\text { Cube } \\
{[43,44]}\end{array}$ & $\begin{array}{c}\text { Thin Square } \\
\text { Plate [44] }\end{array}$ \\
\hline$\kappa_{C A P}$ & 0.5 & 0.66 & 0.36 \\
\hline \hline
\end{tabular}

The Lorentz force acts at a body's effective center-of-charge $\boldsymbol{\delta}_{\boldsymbol{q}}$. The force therefore applies a torque of the form in Eq. 2.10 when the center-of-mass and centerof-charge are not collocated. This torque has been proposed as an attitude actuator for spacecraft capable of controllable charges [45]. For the regular geometries presented here, $\boldsymbol{\delta}_{\boldsymbol{q}}=\underline{\mathbf{0}}$. However, an arbitrarily small deviation in the local charge distribution will produce a torque of the form in Eq. 2.10. In a vacuum environment, the resulting angular acceleration scales with $\lambda^{-3}$.

\subsection{Results}

The acceleration scaling from the above models are summarized in Table 2.6. Here, the magnitude of each acceleration is presented along with the appropriate scale 
factors. With the exception of gravitational accelerations, magnetic attraction, and eddy current damping, each acceleration is somehow dependent on $\lambda$. In each case, the accelerations increase in magnitude as $\lambda$ decreases.

Table 2.6. Acceleration Dependence on $\lambda$ and Geometric Scale Factors

\begin{tabular}{|c|c|c|}
\hline Source & Orbital & Angular \\
\hline Gravity & $\kappa_{V} \lambda^{0}$ & $\kappa_{I} \lambda^{0}$ \\
\hline \multicolumn{3}{|l|}{ Particle Collisions } \\
\hline Aerodynamic Forces & $\frac{\kappa_{A D} \kappa_{C}}{\kappa_{V}} \lambda^{-1}$ & $\frac{\kappa_{A D} \kappa_{C}}{\kappa_{I}} \lambda^{-2}$ \\
\hline Solar Wind & $\underline{\kappa_{C}} \lambda^{-1}$ & $\underline{\kappa_{C}} \lambda^{-2}$ \\
\hline Micrometeoroid Collisions & $\kappa_{V}$ & $\kappa_{I}$ \\
\hline Radiation & & \\
\hline $\begin{array}{l}\text { Solar Radiation Pressure } \\
\text { Planetary Albedo } \\
\text { Thermal Emission }\end{array}$ & $\frac{\kappa_{C}}{\kappa_{V}} \lambda^{-1}$ & $\frac{\kappa_{C}}{\kappa_{I}} \lambda^{-2}$ \\
\hline Poynting-Robertson Drag & $\frac{\kappa_{C}}{\kappa_{V}} \lambda^{-1}$ & - \\
\hline \multicolumn{3}{|l|}{ Magnetic Fields } \\
\hline $\begin{array}{l}\text { Magnetic Attraction } \\
\text { (Dipole Interactions) }\end{array}$ & $\kappa_{V} \lambda^{0}$ & $\frac{\kappa_{V}}{\kappa_{I}} \lambda^{-2}$ \\
\hline Eddy Current Damping & - & $\frac{\kappa_{E C}}{\kappa_{I}} \lambda^{0}$ \\
\hline $\begin{array}{l}\text { Lorentz Force } \\
\text { (Vacuum Environment) }\end{array}$ & $\frac{\kappa_{C A P}}{\kappa_{V}} \lambda^{-2}$ & $\frac{\kappa_{C A P}}{\kappa_{I}} \lambda^{-3}$ \\
\hline
\end{tabular}

Simulations are used to associate these scaling laws with realistic values for the near-Earth environment. The simulations use the Earth Gravity Model (EGM96) [46], the International Geomagnetic Reference Field (IGRF95) [47], and the 1976 Standard Atmosphere Model [48]. Table 2.7 and Table 2.8 give the environmental and spacecraft-specific constants used in the simulations, as well as their references. The spacecraft density is taken to be $79 \mathrm{~kg} / \mathrm{m}^{3}$, the rule-of-thumb density for typical 
spacecraft [49]. The reflective efficiency $\eta$ is taken to be characteristic of a very reflective surface [29]. Two magnetic fields are considered, residual and intentional, denoted by the magnetization term $\Omega_{R}$ and $\Omega_{T}$ respectively. The conductivity $\sigma_{E C}$ is taken to be characteristic of gold traces. The Lorentz force is evaluated for a spacecraft in a retrograde orbit, such that $v_{B}$ is maximized.

Table 2.7. Environmental Constants Used in Simulations

\begin{tabular}{ccc}
\hline Parameter & Value & Ref. \\
\hline$\dot{m}_{P}$ & $6.13 \times 10^{-16} \mathrm{~kg} / \mathrm{m}^{2} \mathrm{~s}$ & {$[16,27]$} \\
$\eta_{p}$ & 0.306 & {$[50]$} \\
$\dot{p}_{S W}$ & $2.3 \times 10^{-9} \mathrm{~kg} / \mathrm{m} \mathrm{s}^{2}$ & {$[28]$} \\
$r_{0}$ & $1 \mathrm{AU}$ & {$[23]$} \\
$T_{p}$ & $255 \mathrm{~K}$ & {$[51]$} \\
$W_{0}$ & $1368 \mathrm{~J} / \mathrm{m}^{2} \mathrm{~s}$ & {$[23]$} \\
$\xi_{p}$ & 1 & \\
\hline \hline
\end{tabular}




\begin{tabular}{ccc}
\multicolumn{3}{c}{ Table 2.8. Spacecraft Constants Used in Simulations } \\
\hline Parameter & Value & Ref. \\
\hline$\eta$ & 0.85 & {$[29]$} \\
$\eta_{a b}$ & 0.1 & \\
$\eta_{d r}$ & 0.1 & \\
$\eta_{n}$ & 0.70 & {$[26]$} \\
$\eta_{t}$ & 0.70 & \\
$\kappa_{\varepsilon}$ & 0.0025 & Table 2.3 \\
$\Omega_{R}$ & $0.5 \mathrm{~A} / \mathrm{m}$ & Table 2.3 \\
$\Omega_{T}$ & $1000 \mathrm{~A} / \mathrm{m}$ & \\
$\omega$ & $0.1 \mathrm{rad} / \mathrm{s}$ & {$[49]$} \\
$\rho$ & $79 \mathrm{~kg} / \mathrm{m}^{3}$ & \\
$\sigma_{E C}$ & $4.2 \times 10^{7}(\mathrm{ohm}-\mathrm{m})^{-1}$ & \\
$\xi$ & 0.85 & \\
$\phi$ & $1000 \mathrm{~V}$ & \\
\hline
\end{tabular}

Table 2.9 is the legend for Figures 2.7 through 2.12. These figures give the magnitude of each of the 14 modeled accelerations as a function of $\lambda$. The accelerations are normalized by the magnitude of Earth's point-mass attraction, and plotted on a log-log scale. Figures 2.7 through 2.9 show each acceleration at altitudes of $500,1,000$, and $10,000 \mathrm{~km}$. In these three figures, the accelerations are unscaled in that each of the unitless scale factors $\kappa_{i}$ (with the exception of $\kappa_{\varepsilon}$ ) in Table 2.6 is set to unity. These plots can be used to provide results specific to any shape of interest.

Figures 2.10 through 2.12 give the scaled accelerations for the three geometries of interest in this analysis. These figures include accelerations due to solar pressure, aerodynamic drag, and the Lorentz force. Each can dominate the non-gravitational dynamics at particular length scales and altitudes. Aerodynamic drag, for example, is 
the largest acceleration at $500 \mathrm{~km}$, while solar pressure is largest at $10,000 \mathrm{~km}$. With a $\lambda^{-3}$ dependence, the Lorentz force becomes the largest as the characteristic length approaches the sub-millimeter scale. Figures 2.10-2.12 suggest that the plate geometry, when aligned for maximum area, experiences the largest effects from nongravitational accelerations, owing to its high $A / m$ ratio: $\kappa_{C} / \kappa_{V}$.

Table 2.9. Acceleration Legend for Figures 2.7 through 2.18 .

\begin{tabular}{cc}
\hline Initials & Acceleration \\
\hline AD & Aerodynamic Drag \\
EC & Eddy Current Drag \\
GG & Gravity Gradient \\
GR & General Relativity \\
LZ & Lorentz Force \\
M & Magnetism \\
MT & Magnetism - Torquer \\
MR & Magnetism - Residual Field \\
Moon & Lunar Gravity \\
OB & Earth Oblateness \\
PA & Planetary Albedo \\
PC & Particle Collisions \\
PL & Solar System Planetary Gravity \\
PR & Poynting-Robertson Drag \\
SP & Solar Pressure \\
SR & Special Relativity \\
SW & Solar Wind \\
Sun & Solar Gravity \\
\hline
\end{tabular}




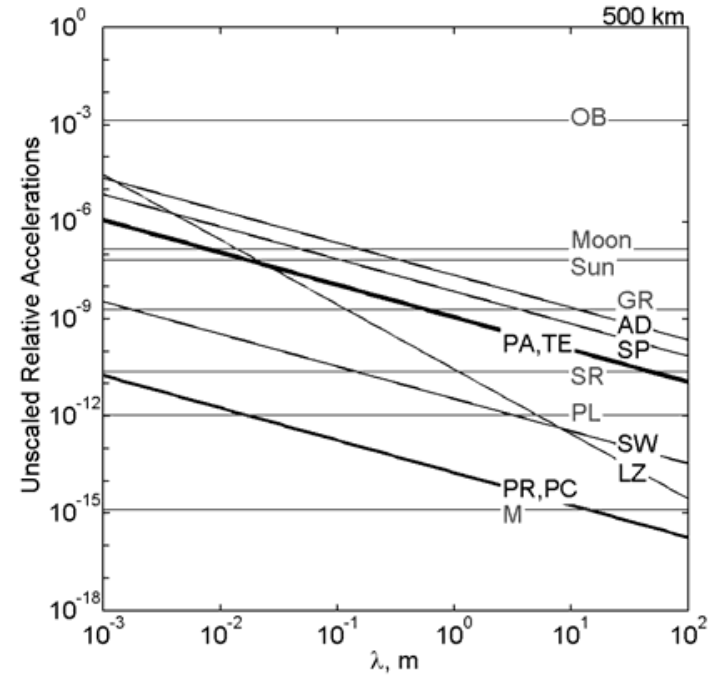

Figure 2.7. Unscaled accelerations at 500 $\mathrm{km}$ altitude as a function of characteristic length, normalized to Earth's two-body gravity.

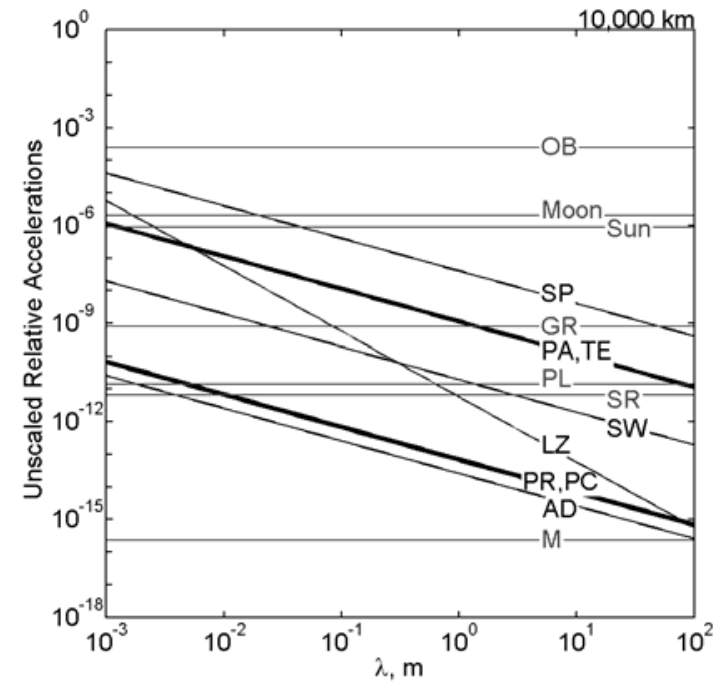

Figure 2.9. Unscaled accelerations at $10,000 \mathrm{~km}$ altitude as a function of characteristic length, normalized to Earth's two-body gravity.

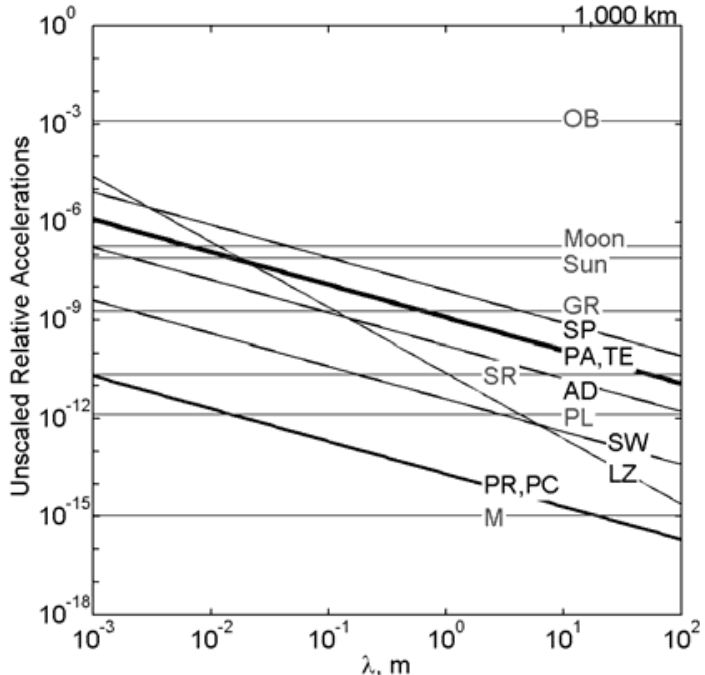

Figure 2.8. Unscaled accelerations at $1,000 \mathrm{~km}$ altitude as a function of characteristic length, normalized to Earth's twobody gravity.

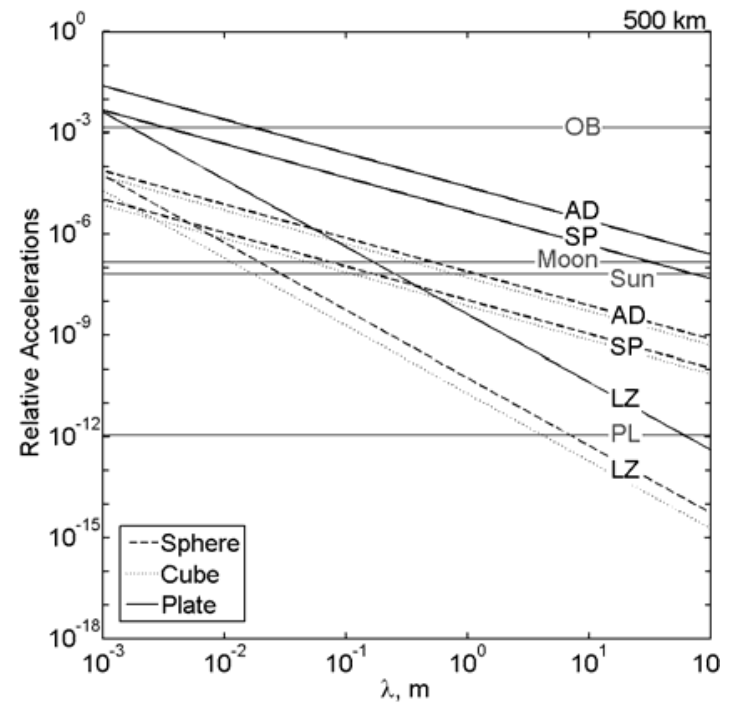

Figure 2.10. Accelerations for a sphere, cube, and plate at $500 \mathrm{~km}$ altitude as a function of characteristic length, normalized to Earth's two-body gravity. 


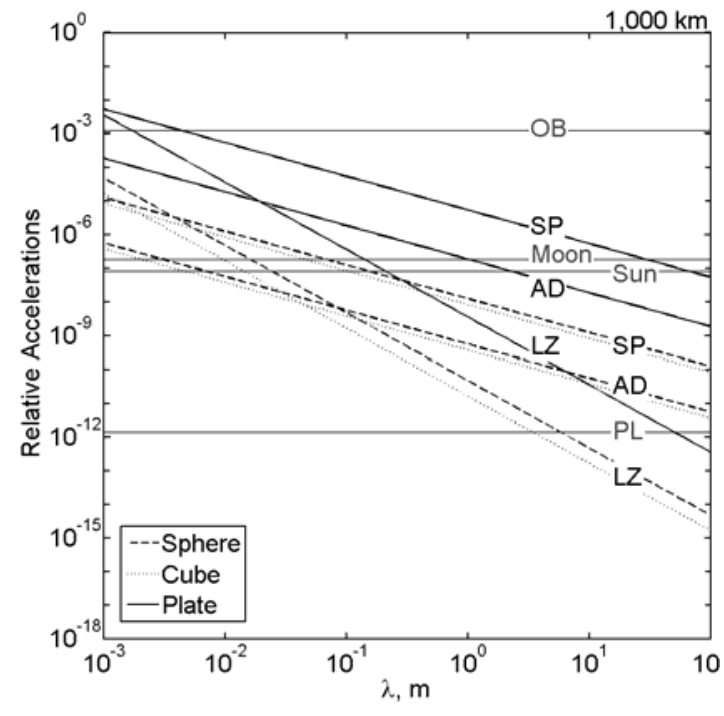

Figure 2.11. Accelerations for a sphere, cube, and plate at $1,000 \mathrm{~km}$ altitude as a function of characteristic length, normalized to Earth's two-body gravity.

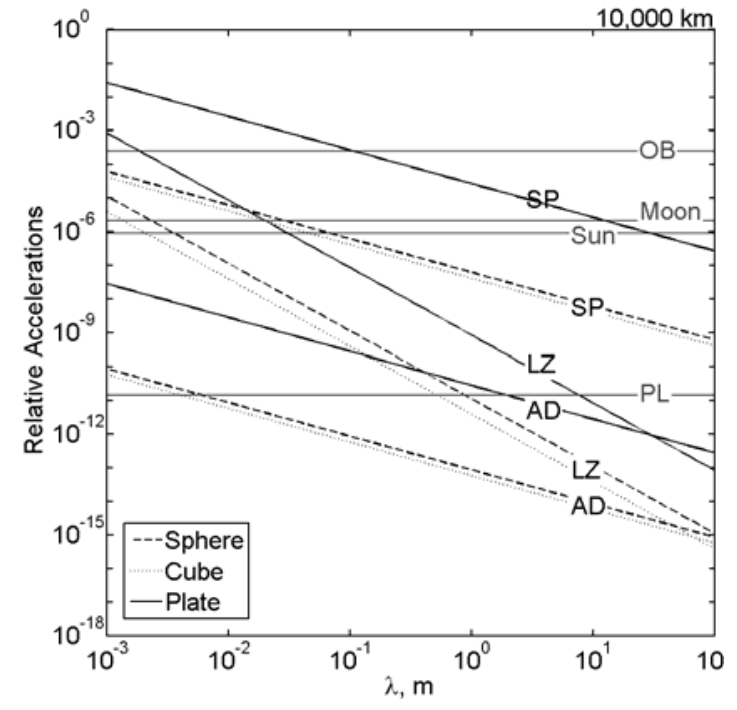

Figure 2.12. Accelerations for a sphere, cube, and plate at $10,000 \mathrm{~km}$ altitude as a function of characteristic length, normalized to Earth's two-body gravity.

Simulations are also used to find the relative magnitudes of angular accelerations. Here, the environmental torques are modeled for the near-Earth environment. Except for gravity gradient, magnetism, and eddy-current torques, all of the environmental torques are a product of an environmental force along a line of action offset from the body's mass center. That is, these torque models take the form of Eq. 2.10, where $\delta$ is taken to be the offset from the mass center. This analysis focuses on the maximum relative magnitude a torque achieves. To this end, Eq. 2.11 is used with $\kappa_{T}$ taken to be a constant, implying that the offset $\delta$ varies with size. For example, given $\kappa_{T}=0.01$, the torques' moment-arm is $1 \%$ of the characteristic length. This assumption may best model manufacturing errors. Two magnetic torques are considered: disturbance 
torques associated with unintentional residual fields (denoted $M R$ ) and actuator torques associated with a magnetic torquer (denoted $M T)$.

Many of the forces or torques are dependent on $A_{C}$. These tend to rotate the plate about a minor axis since the area perpendicular to the major axis $\boldsymbol{b}_{3}$ is negligible. The simulations therefore use the minor moment of inertia.

Figure 2.13 through Figure 2.15 show the unscaled $\left(\kappa_{i}=1\right)$ angular accelerations as a function of characteristic length at altitudes of 500, 1,000, and 10,000 kms. Eddycurrent damping accelerations for $\omega=0.1 \mathrm{rad} / \mathrm{s}$ are length-independent and relatively large. Gravity-gradient accelerations--here due to a displacement of the mass-center by $\delta$--are also length independent. Of the accelerations that scale with $\lambda$, magnetic actuators predictably dominate; after all, this simulation models the entire unscaled body as a single magnetic torquer. As in the orbital-acceleration case, the relative importance of the remaining accelerations can depend on altitude.

Figure 2.16 through Figure 2.18 show the dominant accelerations applied to sphere, cube, and plate geometries. Again, the plate geometry experiences the highest of the non-gravitational accelerations. At LEO altitudes, aerodynamic drag can dominate the angular accelerations for small bodies. At higher altitudes, solar pressure and residual magnetic interactions become increasingly important. The Lorentz force, despite having a $\lambda^{-3}$ dependence, does not overcome these accelerations until submillimeter scales. 


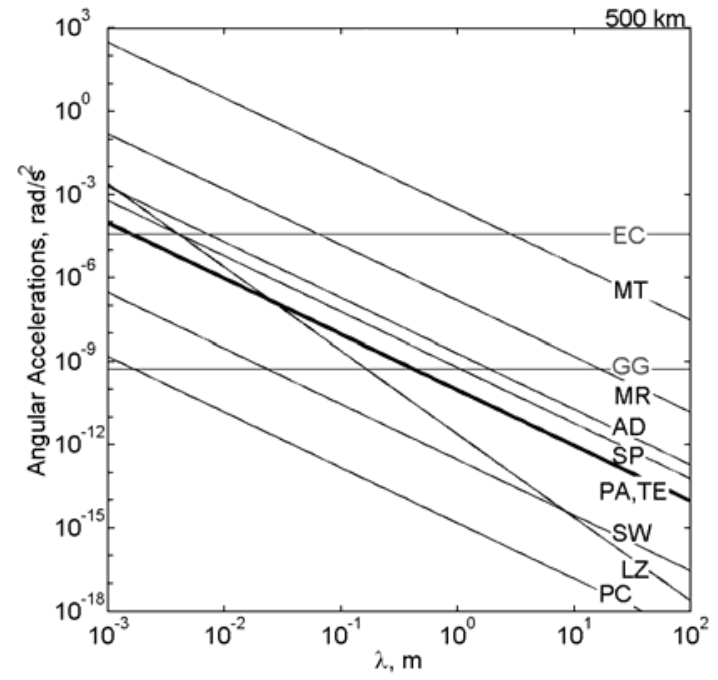

Figure 2.13. Unscaled angular accelerations at $500 \mathrm{~km}$ altitude as a function of characteristic length.

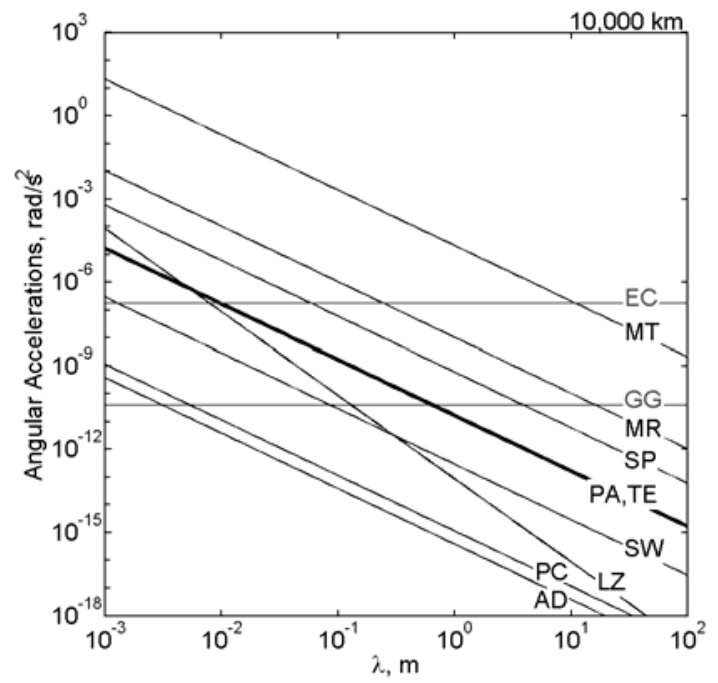

Figure 2.15. Unscaled angular accelerations at $10,000 \mathrm{~km}$ altitude as a function of characteristic length.

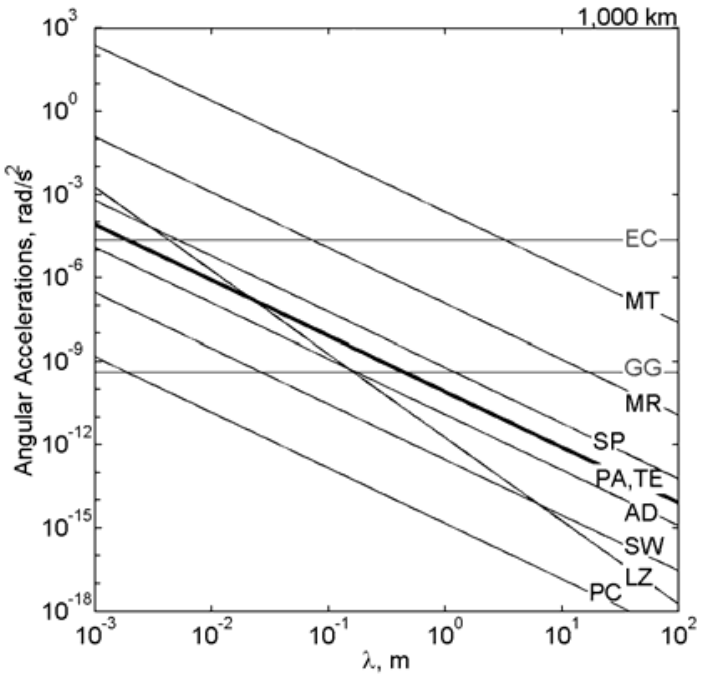

Figure 2.14. Unscaled angular accelerations at $1,000 \mathrm{~km}$ altitude as a function of characteristic length.

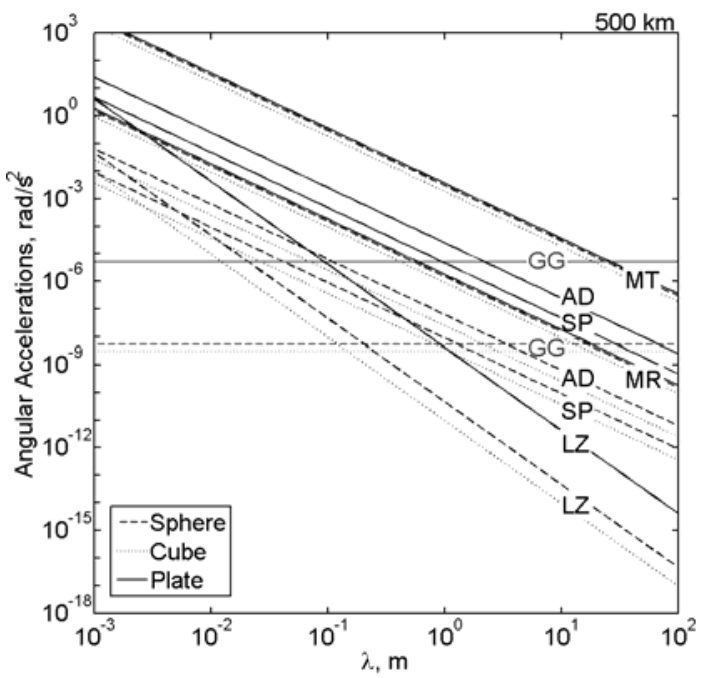

Figure 2.16. Angular accelerations for a sphere, cube, and plate at $500 \mathrm{~km}$ altitude as a function of characteristic length. 


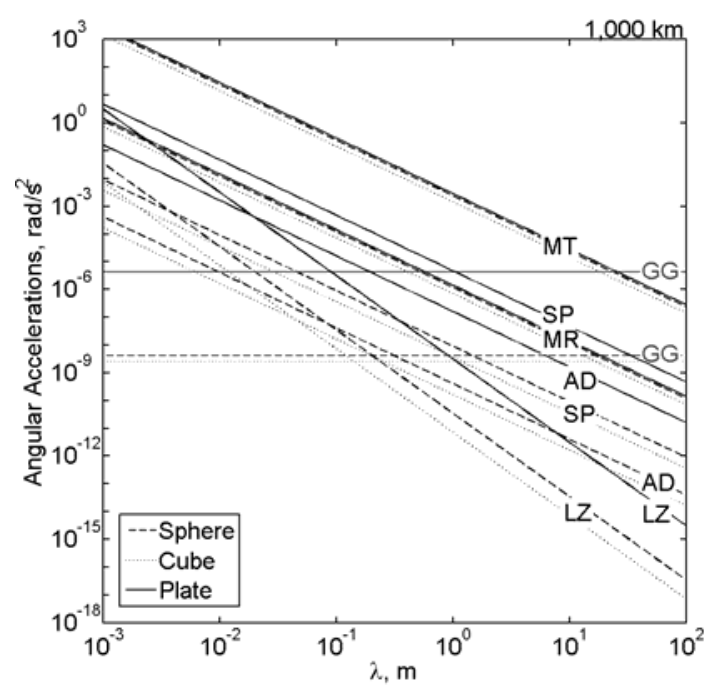

Figure 2.17. Angular accelerations for a sphere, cube, and plate at $1,000 \mathrm{~km}$ altitude as a function of characteristic length.

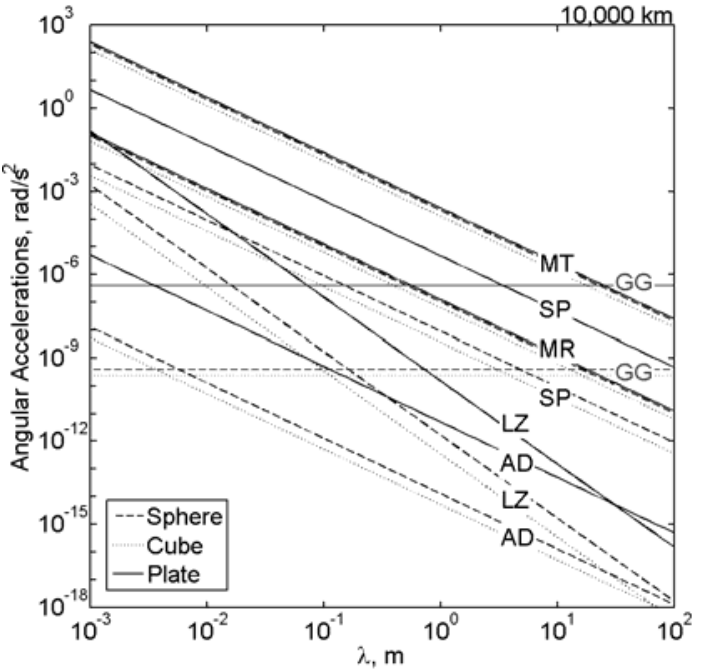

Figure 2.18. Angular accelerations for a sphere, cube, and plate at $10,000 \mathrm{~km}$ altitude as a function of characteristic length.

Previous studies [10,11] have introduced a prototype IC spacecraft-on-chip architecture intended to demonstrate functionality at small $\lambda$. The design consists of a silicon plate with $\lambda=1 \mathrm{~cm}$ and $\kappa_{\varepsilon}=0.0025$. Taking $\delta=1 \mathrm{~mm}$, Figure 2.19 and Figure 2.20 show simulations of this architecture in the near-Earth environment. Each of the variables is plotted as a function of altitude. 

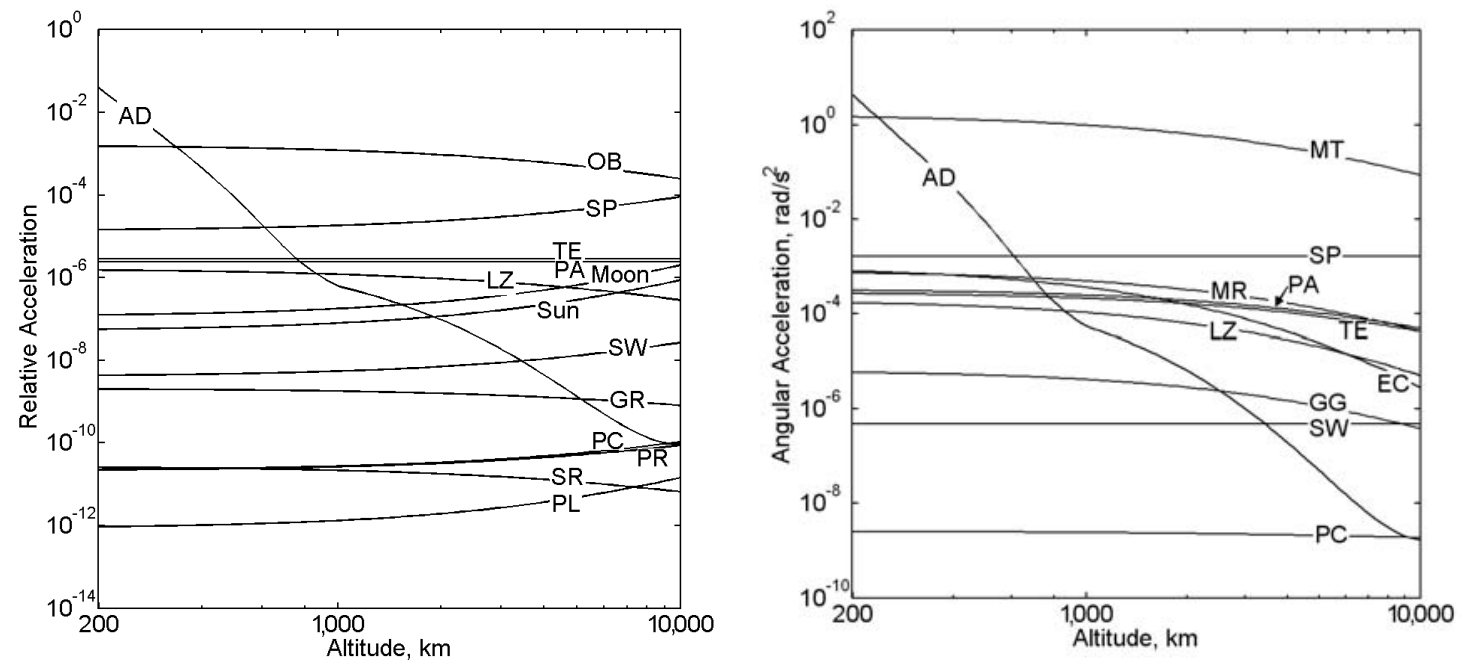

Figure 2.19. Accelerations acting on a Figure 2.20. Angular accelerations acting candidate spacecraft-on-chip architecture, on a candidate spacecraft-on-chip archinormalized to Earth's two-body gravity, tecture as a function of altitude. and given as a function of altitude.

\subsection{Discussion}

These results have implications for small spacecraft design, though there are obvious limits in their interpretation. Specifically, constant spacecraft density likely does not apply across all length scales. It also may not be realistic to model a plate as having a constant $\kappa_{\varepsilon}$ across all $\lambda$. Despite these limitations, this analysis inspires relevant applications. The candidate spacecraft-on-chip used in the above analysis is designed with a $1 \mathrm{~cm}$ characteristic length in order to enable new mission opportunities based on non-gravitational accelerations. This discussion briefly considers three such opportunities for the candidate bus: solar sailing, aerodynamic reentry, and Lorentz propulsion.

In each of these scenarios, the orbit of an extremely small spacecraft bus is sensitive to a particular non-gravitational acceleration. In terms of functionality, it 
may be that the limitations of a single spacecraft-on-chip can be overcome by deploying large numbers of spacecraft. Individually, each unit would experience nonKeplerian dynamics and offer low bandwidth sensor data. As a single swarm, the combined collection of sensors would offer a spatially rich dataset that couldn't be reproduced by a single larger spacecraft. In this scenario, forces or interactions between the spacecraft-on-chips are negligible compared to the environmental forces considered in this research.

\subsubsection{Solar Sail}

A solar sail provides a means to utilize solar radiation pressure as a means of propellantless propulsion. This analysis suggests that solar pressure can be achieved with small, thin plate-like structures. In fact, there are additional benefits to small size when considering solar sail architectures. Typical sail designs are extremely large and challenging to construct, deploy, and actuate. Greschik [53] suggests that dimensional challenges are primarily responsible for the as yet unsuccessful solar-sail tests, despite thirty years of attempts. The range of magnitudes involved in solar sails make structural analyses intractable, fabrication demanding, and ground testing extremely challenging. These issues have motivated the development of small sized architectures including solar kites [54], microsolar sails [55], and nanosails [56].

For interplanetary dust, solar radiation pressure can exceed gravity. Here, the critical radius of a particle is roughly a tenth of a micron, below which the particle can be too small to absorb or reflect the photon [10]. Highly reflective interplanetary dust particles of this size can escape solar gravity if released from a comet near the sun [2]. These so-called $\beta$ meteoroids were most recently detected by the Ulysses [57] and Galileo [58] spacecraft. 
This analysis indicates that the candidate spacecraft-on-chip architecture can capitalize on length scaling to achieve significant solar pressure acceleration. That is, the bus itself, by virtue of its geometry, behaves as a solar sail. The millimeter-scale design can be fabricated using integrated-circuit techniques and can be readily tested in a $1 \mathrm{G}$ environment. Further, by capitalizing on natural dynamics, it may be capable of avoiding the nontrivial challenges associated with solar-sail control and actuation. Previous research [10] has evaluated this concept, accounting for passive attitude control mechanisms and proposing sample missions concepts.

A common metric for solar sail designs is the lightness number $\beta_{S P}$, which compares the solar pressure acceleration to solar gravitation [29]

$$
\beta_{S P} \equiv \frac{a_{S}}{a_{G}}=2 \frac{W_{0} r_{0}^{2}}{c \mu \rho} \frac{\eta \kappa_{C}}{\kappa_{V}} \lambda^{-1} .
$$

This metric accurately describes the acceleration's dependence on body size for lengths above the wavelength of visible light. The candidate silicon spacecraft-onchip bus achieves a lightness number of 0.01 , meaning that the magnitude of solar pressure is $1 \%$ of solar gravity. Though this value is smaller than many proposed solar sail designs, there are a number of possible applications for it in geocentric, heliocentric, or three-body orbits as explored in previous research [10]. Even thinner bodies retain the advantages of stiffness and ready deployment, and they would better compete with the lightness number of larger sails.

\subsubsection{Reentry Dynamics}

Like most of the non-gravitational orbital accelerations, atmospheric drag depends on the area-to-mass ratio $A_{C} / m$. The inverse of this ratio appears in the commonly used ballistic coefficient, defined to be a ratio of inertia to aerodynamic drag [59] 


$$
\beta_{A D} \equiv \frac{m}{\kappa_{A D} A_{C}}=\frac{\kappa_{V} \rho}{\kappa_{A D} \kappa_{C}} \lambda
$$

This ratio determines a body's drag-limited lifetime in LEO. Low values of $\beta_{A D}$ correspond to satellites whose orbits are highly affected by atmospheric drag, and consequently deorbit more quickly than bodies with high $\beta_{A D}$. Typical spacecraft have ballistic coefficients on the order of 10 to $100 \mathrm{~kg} / \mathrm{m}^{2}$ [60]. When face-on to the flow $\left(\gamma_{A}=\pi / 2\right)$, the candidate spacecraft-on-chip bus has a ballistic coefficient of 0.023 $\mathrm{kg} / \mathrm{m}^{2}$.

Figure 2.20 shows that acceleration associated with magnetic actuator torque is greater than those associated with atmospheric drag. This feature suggests that a magnetic torquer could align the attitude of a plate with the magnetic field when commanded, enabling a form of controlled aerobraking or reentry.

A primary challenge for spacecraft reentry maneuvers is heat management, where both the rate and total load of heat can cause catastrophic failure. Essentially, aerodynamic drag converts the spacecraft's kinetic energy to thermal energy. A spacecraft must be capable of both decelerating and shedding heat rapidly enough to survive reentry. In their assessment of the survivability of small orbital debris, Koppenwallner, Fritsche, and Lips [59] develop model for reentry that explicitly considers $\lambda$. Characteristic length enters the model through two of the three aerodynamic similarity parameters: Reynolds number and Knudsen number [59]:

$$
\begin{gathered}
R e=\frac{\rho_{A D} v}{\mu_{A D}} \lambda \\
K n=\frac{\varsigma}{\lambda}
\end{gathered}
$$

where $\mu_{A D}$ is the local atmospheric fluid viscosity and $\zeta$ is the mean free path of the atmospheric gases. The third similarity parameter, the Mach number, depends on only 
velocity and local fluid properties. These three parameters define the flow environment and heat transfer regime of the reentering body. Free-molecular flow is defined by $K n>10$, and hypersonic continuum flow is defined by $K n<0.1$. Intermediate values are characterized as transitional flow and are approximated with some choice of bridging function. The heat imparted to the reentry body is modeled by [59]

$$
\dot{Q}_{\text {aero }}=\frac{1}{2} S t \rho_{A D} v^{3} A_{C},
$$

where the Stanton number St varies according to the flow regime. The heat input is therefore a function of $\lambda^{2}$ explicitly, with an additional, though less clear, dependence on $\lambda$ through the fluid similarity parameters. Further, the back half of the spacecraft can radiate heat $Q_{r a d}^{\circ}$ to and from the surrounding planet (with temperature $T_{p}$ ) according to the Stephan-Boltzmann law given by Eq. 2.36. As before, this radiation term is a function of $\lambda^{2}$.

Given these models for heat transfer, the temperature of a spacecraft with specific heat capacity $c_{p}$ follows the first-order differential equation [59]

$$
\dot{T}=\frac{\dot{Q}_{a e r o}+\dot{Q}_{r a d}}{m c_{p}} .
$$

Thus the ratio of $A / m$ also appears in the heat transfer equation, which suggests that the temperature of small bodies is more sensitive to heat rates. It turns out that dust particles can survive re-entry at low temperatures thanks to their small size [6,7]. Aerodynamic drag decelerates the dust particles to subsonic velocities in the upper atmosphere where the density is low and aerothermal heat rates are very low. Each year, thousands of metric tons of small interplanetary dust particles reach the Earth's surface unaffected while larger meteoroids energetically ablate as meteorites [61]. 
This drag and thermal model was simulated for a proposed spacecraft-on-chip architecture (a square flat plate with $\lambda=1 \mathrm{~cm}$ and $\kappa_{\varepsilon}=0.0025$ ) from an altitude of 350 $\mathrm{km}$. The results of this simulation are given in Figure 2.21. For the first orbit, the thin square plate is kept edge-on to the flow and drag is minimal. Once commanded, the attitude is taken to be face-on to the velocity, such that drag is maximized. The altitude rapidly drops and the temperature increases to a peak value of only about $105^{\circ}$ C during maximum deceleration, after which it settles to a steady state temperature driven by the Earth's thermal radiation. This peak heating occurs in the freemolecular flow regime and results in temperatures low enough to suggest that an IC could operate throughout reentry. There may be meaningful mission opportunities for a small sensor that can sample many altitudes of the atmosphere continuously throughout the reentry process, and one that furthermore would not experience the plasma-related communications dropout of hotter reentering spacecraft.

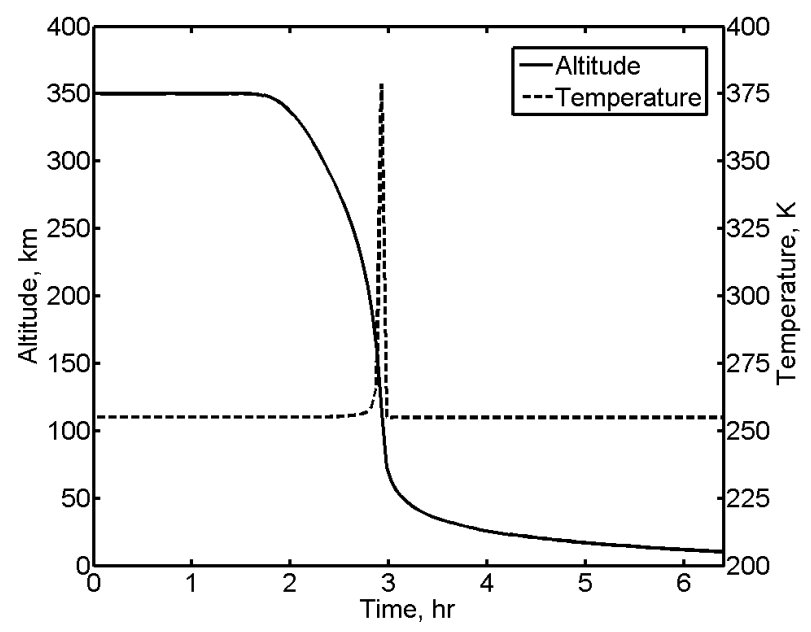

Figure 2.21. Time-history of altitude and temperature for a simulated reentry maneuver of a candidate spacecraft-on-chip spacecraft. 


\subsubsection{Lorentz Force Spacecraft}

The Lorentz force is responsible for capturing and ejecting electrostatically charged dust particles in the rings of Jupiter $[62,63,64]$ and Saturn $[65,66]$. The dust particles attain time-varying charges that produce non-Keplerian orbits with altered orbital energy. If charge can be artificially generated on a spacecraft, it could serve as a means of propulsion. Previous research has explored the novel spacecraft maneuvers such a technology could enable $[42,67,68,69]$. Though a few architectures have been proposed to accommodate the self-capacitance and potential required for meaningful maneuvers, they require relatively large structures, such as groups of kilometer-long filaments. Based on this analysis and previous research [11], the $\lambda^{-3}$ scaling of $q / m$ implies that Lorentz force may instead be most easily achieved using a very small spacecraft.

Power represents a design challenge for equipping a spacecraft to propel itself via the Lorentz force. The spacecraft must produce enough power to maintain a net charge in spite of the near-Earth plasma environment that tends to discharge charge imbalances. The plasma environment discharges the spacecraft through so-called thermal and ram currents. These currents are functions of plasma characteristics, as well as the area of the charged spacecraft. That is, power requirements reduce according to an area-to-mass scaling. At small enough characteristic lengths, the discharge currents are characterized by the orbital-motion-limited regime, which reduces the power requirements even further.

A promising architecture proposed by Hoyt and Minor [70] requires only a power source and two plasma contactors to achieve a net charge. The following thought experiment, illustrated in Figure 2.22, explains the concept. In a vacuum, if two conductive wires are connected to the terminals of a potential source (e.g. a 
battery or a solar cell), each wire can be thought of as reaching a potential equal to half of the source's potential and with opposing polarities. However, in a plasma environment, the wires' opposite polarities generate dissimilar plasma currents, resulting in dissimilar wire potentials [71]. Near-Earth, the positive wire would tend to discharge almost entirely. As long as the power source can supply sufficient current, the source would maintain the potential difference and would drive the potential to the negative wire. Such a spacecraft would carry a net negative charge that may be controlled through the power source.
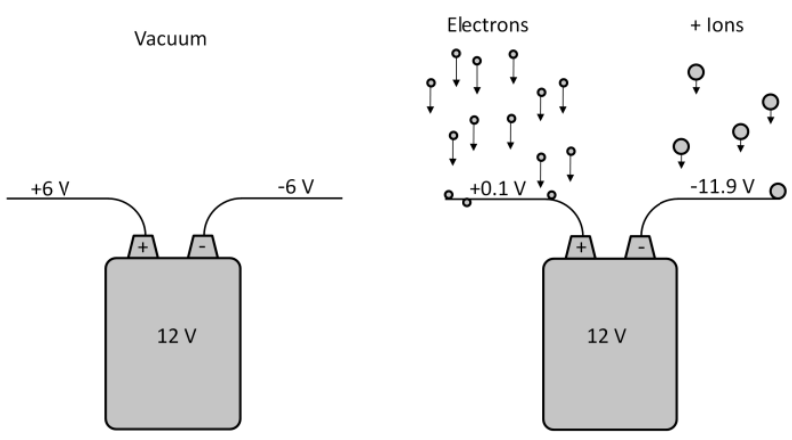

Figure 2.22. Equilibrium potentials for battery terminals in a vacuum and plasma environment.

Using this architecture, the candidate spacecraft-on-chip bus could be equipped for Lorentz propulsion using only two thin, conductive wires and a small solar-cell array. The wires act as plasma contactors and capacitors, and the solar cells produce the potential difference and current required to overcome plasma discharge currents. A previous study suggests that the candidate spacecraft-on-chip bus attached with two $1 \mathrm{~m}$ long wires and solar cells can achieve a $q / m$ on the order of a micro-Coulomb per kilogram. For a $500 \mathrm{~km}$ orbit, this $q / m$ is sufficient to produce daily growth of roughly $400 \mathrm{~m}$ of semimajor axis or $25 \mathrm{~m}$ of along-track motion. 


\subsection{Conclusion}

The magnitude of orbital and angular accelerations in the near-Earth space environment can be highly dependent on the characteristic length $\lambda$ of the affected body. Most near-Earth perturbations can be modeled as pressures, in which case the critical ratio is the area-to-mass, which is dependent on $\lambda^{-1}$. The Lorentz force is dependent on the charge-to-mass ratio, which scales with $\lambda^{-2}$ in a vacuum. As $\lambda$ is reduced, these orbital and attitude accelerations become increasingly large, while gravitational accelerations remain unaffected. This understanding suggests which environmental forces and torques can and cannot be neglected for a given simulation and error tolerance. There are regimes of characteristic length and altitude, within which different accelerations dominate the dynamics of a spacecraft.

If $\lambda$ is sufficiently small, non-gravitational accelerations may reach magnitudes sufficient to enable new mission opportunities. By designing a spacecraft to have a very small $\lambda$, mission designers can potentially achieve novel spacecraft maneuvers passively and without the requirement of on-board fuel.

A second design parameter is choice of geometry. This research offers a framework for considering a spacecraft geometry using non-dimensional scale factors. Of the geometries considered here, a sphere and cube have fixed scale factors, while the scale factors for a square flat plate depend on thickness.

A $1 \mathrm{~cm}$ scale candidate spacecraft-on-chip bus is considered as a solar sail, a reentry vehicle, and a Lorentz propelled spacecraft. In each case, the magnitude of nongravitational acceleration suggests the potential for meaningful propellantless maneuvers. Swarms of small spacecraft could conceivably offer new mission opportunities, with each individual unit experiencing large non-gravitational accelerations. 


\subsection{Acknowledgments}

The authors wish to thank Zac Manchester, Sharon Kotz, Robert MacCurdy, and Alfred Ernst of Cornell University for their many contributions to this work. The authors also wish to thank Sandia National Laboratory's Center for Integrated Nanotechnology for their invaluable collaboration, expertise, and support. 


\section{REFERENCES}

[1] Harwit, M., "Origins of the Zodiacal Dust Cloud," Journal of Geophysical Research, Vol. 68, No. 8, 1963, pp. 2171-2180.

[2] Burns, J., Lamy, P., and Soter, S., "Radiation Forces on Small Particles in the Solar System," Icarus, Vol. 40, 1979, pp. 1-48.

[3] Kresak, L., "Orbital Evolution of the Dust Streams Released from Comets," Astronomical Institutes of Czechoslovakia Bulletin, Vol. 27, No. 1, 1976, pp. 35-46.

[4] Colwell, J.E., Horanyi, M., and Grun, E., "Capture of Interplanetary and Interstellar Dust by the Jovian Magnetosphere", Science, Vol. 280, April 1998, pp. 88-91.

[5] Hamilton, D.P. and Burns, J.A., "Ejection of Dust from Jupiter's Gossamer Ring", Nature, Vol. 364, August 1993, pp. 695-699.

[6] Whipple, F.L., "Theory of micro-meteorites. I. In an isothermal atmosphere." Proceedings of the National Academy of Sciences USA, Vol. 86, 1950, pp. 687-695.

[7] Beech, M., "Finite-size Corrections to the Atmospheric Heating of Micrometeorites", Monthly Notices of the Royal Astronomical Society, Vol. 402, No. 2, 2010, pp. 1208-1212.

[8] Barnhart, D., Vladimirova, T., Sweeting, M., "Very-Small-Satellite Design for Distributed Space Missions," Journal of Spacecraft and Rockets, Vol. 44, No. 6, 2007, pp. 1294-1306.

[9] Barnhart, D., Vladimirova, T., Sweeting, M., "Satellite Miniaturization Techniques for Space Sensor Networks", Journal of Spacecraft and Rockets, Vol. 46, No. 2, 2009, pp. 469-472.

[10] Atchison, J.A., and Peck, M.A., "A Passive, Sun-Pointing, Millimeter-Scale Solar Sail", Acta Astronautica, Spring 2010, In Press.

DOI:10.1016/j.actaastro.2009.12.008

[11] Atchison, J.A., and M.A. Peck, "A Millimeter-Scale Lorentz-Propelled Spacecraft", AIAA Guidance, Navigation, and Control Conference and Exhibit, AIAA2007-6847, South Carolina, August 20-23, 2007. 
[12] Janson, S.W., "Mass-Producible Silicon Spacecraft for 21st Century Missions," AIAA Space Technology Conference \& Exposition, Albuquerque, NM, 1999.

[13] Janson, S.W., Helvajian, H., Breuer, K., "MEMS, Microengineering and Aerospace Systems", 30th AIAA Fluid Dynamics Conference, AIAA A99-33749, Norfolk, VA, 1999, pp. 1-12.

[14] Janson, S.W., "Micro/Nanotechnology for Micro/Nano/Picosatellites", Space 2003, AIAA 2003-6269, Long Beach, CA, 2003, pp. 1-11.

[15] Miller, L.M., "MEMS for space applications," Proceedings of Society of Photographic Instrumentation Engineers (SPIE), Vol. 3680, Paris, April 1999, pp. 113.

[16] Longuski, J.M., Todd, R.E., and König, W.W., "Survey of Nongravitational Forces and Space Environmental Torques: Applied to the Galileo", Journal of Guidance, Control, and Dynamics, Vol. 15, No. 3, 1992, pp. 545-553.

[17] Heidt, H., Puig-Suari, J., Moore, A.S., Nakasuka, S., and R.J. Twiggs, "Cubesat: A new Generation of Picosatellite for Education and Industry Low-Cost Space Experimentation", AIAA Paper SSC00-V-5, AIAA/Utah State University Conference on Small Satellites, August 2001.

[18] Hughes, P.C., "Chapter 3: Attitude Motion Equations”, Spacecraft Attitude Dynamics, Dover Publications, New York, 2004, pp. 39-83.

[19] Hughes, P.C., "Chapter 8: Spacecraft Torques”, Spacecraft Attitude Dynamics, Dover Publications, New York, 2004, pp.233-280.

[20] Vallado, D. A., "Chapter 1.4: Three-body and n-body Equations", Fundamentals of Astrodynamics and Applications, $2^{\text {nd }}$ Ed., Microcosm Press, El Segundo, CA, 2004, pp. 33-48.

[21] McCarthy, D.D. and Petit, G., Editors, "Chapter 10: General Relativistic Models for Space-Time Coordinates and Equations of Motion”, International Earth Rotation and Reference Systems Service, Technical Note No. 32, 2003.

[22] Mashoon, B., Helh, F.W., and Theiss, D.S., "The Lense-Thirring Papers", General Relativity and Gravitation, Vol. 16, pp. 711-750, 1984. Translation of Original Paper: Lense, J., and Thirring, H., "On the Influence of the Proper Rotation of Central Bodies on the Motions of Planets and Moons According to Einstein's Theory of Gravitation", Zeitschrift fur Physik, Vol 19, 2918, pp.156-163. 
[23] Vallado, D. A., "Chapter 8.6: Disturbing Forces", Fundamentals of Astrodynamics and Applications, $2^{\text {nd }}$ Ed., Microcosm Press, El Segundo, CA, 2004, pp. 550551.

[24] King-Hele, D.G., "Chapter 2.3: Evaluation of Drag Coefficient", Satellite Orbits in an Atmosphere: Theory and Application, Blackie and Sons, Glasgow, 1987.

[25] Cook, G.E., "Satellite Drag Coefficients", Planetary Space Science, Vol. 13, 1965, pp.929-946.

[26] Storch, J.A., "Aerodynamic Disturbances on Spacecraft in Free-Molecular Flow", The Aerospace Corporation, Report No TR-2003(3397)-1, October 2002, pp 170.

[27] Cour-Palais, B.G., "Meteoroid Environment Model - 1969 [Near Earth to Lunar Surface]", NASA SP-8013, March 1969.

[28] Wertz, J.R., "Chapter 5.3 Solar Radiation and the Solar Wind", Spacecraft Attitude Determination and Control, D.Reidel, Dordrecht, Holland, 1978, pp. 129132.

[29] McInnes, C.R., "Solar Sailing: Technology, Dynamics and Mission Applications," Springer Verlag, 1999, pp. 1-296.

[30] Blanco, V.M., and McCuskey, S.W., Basic Physics of the Solar System, AddisonWesley, Reading, MA, 1961, pp. 50-52.

[31] Antreasian, P.G., and Rosborough G.W., "Prediction of Radiant Energy Forces on the TOPEX/POSEIDON Spacecraft", Journal of Spacecraft and Rockets, Vol. 29, No. 1, 1992, pp. 1-10.

[32] H.P. Robertson, "Dynamical Effects of Radiation in the Solar System", Monthly Notices of the Royal Astronomical Society, Vol. 97, No. 6, 1937, pp. 423-438.

[33] Griffins, D.J., "Chapter 6.2: The Field of a Magnetized Object", Introduction to Electrodynamics, $3^{\text {rd }}$ Edition, 1999, pp. 263-268.

[34] Yung, K.W., Landecker, P.B., and Villani D.D., "An Analytic Solution for the Force Between Two Magnetic Dipoles", Magnetic and Electrical Separation, Vol. 9, No 1., 1998, pp. 39-52. 
[35] Parker, R.J., "Ch. 6.7: Permanent Magnets and the Laws of Electromagnetic Scaling", Advances in Permanent Magnetism, John Wiley and Sons, Canada, 1990, pp. 179-181.

[36] Parker, R.J., "Appendix 2: Magnetic and Physical Property Tables", Advances in Permanent Magnetism, John Wiley \& Sons, New York, 1990, 316-322.

[37] Schalkowsky, S., and Harris, M., "Spacecraft Magnetic Torques", NASA Space Vehicle Design Criteria, NASA SP-8018, March 1969, pp. 1-51.

[38] Eterno, J.S., "Chapter 11.1: Attitude Determination and Control", Space Mission Analysis and Design, 3rd Ed., 2005, Microcosm Press, El Segundo, CA, p 354381.

[39] Griffins, D.J., "Chapter 7: Electrodynamics", Introduction to Electrodynamics, $3^{\text {rd }}$ Edition, 1999, pp. 263-268.

[40] Wilson, R.H., "Rotational Magnetodynamics and Steering of Space Vehicles", NASA Technical Note, TN D-566, September 1961, pp. 1-23.

[41] Sehnal, L. "The Motion of a Charged Satellite in the Earth's Magnetic Field", Smithsonian Astrophysical Observatory Report, No 271, April 1969, pp. 1-15.

[42] Streetman, B., and Peck, M., "New Synchronous Orbits Using the Geomagnetic Lorentz Force", Journal of Guidance, Control, and Dynamics, Vol. 30, No. 6, 2007, pp. 1677-1690.

[43] Hwang, C. and Mascagni, M., "Electrical Capacitance of the Unit Cube", Journal of Applied Physics, Vol. 95, No. 7, April 2004, pp. 3798-3902.

[44] Read, F.H., "Capacitances and Singularities of the Unit Triangle, Square, Tetrahedron and Cube", Journal of Electrostatics, Vol. 62, No. 1, September 2004, pp. 51-62.

[45] Tikhonov, A. A., "A Method of Semipassive Attitude Stabilization of a Spacecraft in the Geomagnetic Field," Cosmic Research (Translation of Kosmicheskie Issledovaniya), Vol. 41, No. 1, 2003, pp. 63-73. DOI:

[46] Lemoine, F.G., Kenyon, S.C., Factor, J.K., Trimmer, R.G., Pavlis, N.K., et al, "The Development of the Joint NASA GSFC and NIMA Geopotential Model EGM96", NASA Technical Publication, NASA/TP-1998-206861, Greenbelt, Maryland, July 1998. 
[47] C. E. Barton, "International Geomagnetic Reference Field : The Seventh Generation," Journal of Geomagnetism and Geoelectricity, Vol. 49, No. 2, 1997, pp. $123-148$.

[48] NASA, “U.S. Standard Atmosphere 1976”, NASA Technical Report, TM-X74335, October 1976.

[49] Reeves E.I., "Spacecraft Design and Sizing”, Space Mission Analysis and Design, Kluwer Academic Pub, 1999, pp.301-352.

[50] Cole, G.H.A., Woolfson, M.M., "Planetary Albedoes", Planetary Science: The Science of Planets Around Stars, Institute of Physics Publishing, London, 2002, pp. 380-382.

[51] Gilmore, D.G., Hardt, B.E., Prager, R.C., Grob, E.W., and Ousley W., "Chapter 11.5: Thermal", Space Mission Analysis and Design, 3rd Ed., 2005, Microcosm Press, El Segundo, CA, p 433.

[52] Bauccio, M., "Electrical Resistivity of Metals", ASM Metals Reference Book, $3^{\text {rd }}$ Ed, ASM International, Materials Park, OH, 1993, pp. 157-158.

[53] Greschik, G., "Solar Sail Scalability and a 'Truly Scalable' Architecture: The Space Tow," Journal of Spacecraft and Rockets, Vol. 44, No. 4, 2007, pp. 831839.

[54] Jack, C., Welch, C.S., "Solar Kites: Small Solar Sails With No Moving Parts", Acta Astronautica, Vol. 40, No. 2-8, 1997, pp. 137-142.

[55] Lappas, V., Wie, B., McInnes, C.R., Tarabini, L., Gomes, L., and K. Wallace, "Microsolar Sails for Earth Magnetotail Monitoring", Journal of Spacecraft and Rockets, Vol. 44, No. 4, 2007, pp. 840-848.

[56] Prucey, R., Morcone, J., and Murphy, D., "NASA Nanosatellites Catch Ride on Rocket, Demonstrate Technology", NASA Press Release: H-08-70AR, July 31, 2008.

[57] Wehry, A., and Mann, I., "Identification of $\beta$-meteoroids from Measurements of the Dust Detector Onboard the Ulysses Spacecraft," Astronomy and Astrophysics, Vol. 341, No. 1, 1999, pp. 296-303.

[58] Berg, O., and Grun, E., "Evidence of Hyperbolic Cosmic Dust Particles," Space Research XIII, 1973, pp. 1047-1055. 
[59] Koppenwallner, G., Fritsche, B., and Lips, T. "Survivability and Ground Risk Potential of Screws and Bolts of Disintegrating Spacecraft During Uncontrolled ReEntry" Proceedings of the Third European Conference on Space Debris, ESA SP473, 2001, pp 533-539.

[60] Griffin, M.D., and J.R. French, “Chapter 11 Atmospheric Entry”, Space Vehicle Design, $2^{\text {nd }}$ Ed., AIAA, Reston VA, pp 305-320.

[61] Love, S.G., and D.E. Brownlee, "A Direct Measurement of the Terrestrial Mass Accretion Rate of Cosmic Dust", Science, Vol. 262, No. 5133, October 1993, pp. 550-553.

[62] Grun, E., Kruger, H., Graps, A.L., Hamilton, D.P., Heck, A., et al, “Galileo Observes Electromagnetically Coupled Dust in the Jovian Magnetosphere", Journal of Geophysical Research, Vol. 103, No. E9, 1998, pp. 20011-20022.

[63] Colwell, J.E., Horanyi, M., and Grun, E., "Capture of Interplanetary and Interstellar Dust by the Jovian Magnetosphere”, Science, Vol. 280, April 1998, pp. 88-91.

[64] Schaffer, L. and Burns, J.A., "The Dynamics of Weakly Charged Dust: Motion through Jupiter's Gravitational and Magnetic Fields," Journal of Geophysical Research, Vol. 92, 1987, pp. 2264-2280.

[65] Horanyi, M., "Charged Dust Dynamics in the Solar System," Annual ReviewsAstronomy and Astrophysics, Vol. 34, 1996, pp. 383-418.

[66] Smith, B.A., Soderblom, L.A. , Beebe, R.F. , Boyce, J., Briggs, G.A., et al., 1982. "Encounter with Saturn: Voyager 1 Imaging Science Results," Science, Vol. 212, No. 4491, 1982, pp. 163-191.

[67] Streetman, B. and Peck, M., "A General Bang-Bang Control Method for Lorentz Augmented Orbits," AAS 08-111, AAS Spaceflight Mechanics Meeting, Galveston, TX, Jan 27-31, 2008.

[68] Atchison, J.A. and Peck, M., “Lorentz Augmented Jovian Orbit Insertion.” Journal of Guidance, Control, and Dynamics, Vol. 32, No. 2, March 2009, pp. 418423.

[69] Streetman, B. and Peck, M., "Gravity-Assist Maneuvers Augmented by the Lorentz Force," Journal of Guidance, Control, and Dynamics, Vol 32, No. 5, 2009, pp. 1639-1647. 
[70] Hoyt, R. P., and Minor, B. M., "Remediation of Radiation Belts Using Electrostatic Tether Structures," Tethers Unlimited Inc., 2004.

[71] Hillard G. B. and Ferguson, D. C., "Low Earth Orbit Spacecraft Charging Design Guidelines," NASA Technical Publication, NASA/TP-2003-212287, February 2003. 


\section{CHAPTER 3}

\section{A PASSIVE, SUN-POINTING, MILLIMETER-SCALE SOLAR SAIL*}

\subsection{Abstract}

Taking inspiration from the orbital dynamics of dust, we find that spacecraft length scaling is a means of enabling infinite-impulse orbits that require no feedback control. Our candidate spacecraft is a $25 \mu \mathrm{m}$ thick, $1 \mathrm{~cm}$ square silicon chip equipped with signal transmitting circuitry. This design reduces the total mass to less than $7.5 \mathrm{mg}$ and enables the spacecraft bus itself to serve as a solar sail with characteristic acceleration on the order of $0.1 \mathrm{~mm} / \mathrm{s}^{2}$. It is passive in that it maneuvers with no closed-loop actuation of orbital or attitude states. The unforced dynamics that result from an insertion orbit and a launch-vehicle separation determine its subsequent state evolution. We have developed a system architecture that uses solar radiation torques to maintain a sun-pointing heading and can be fabricated with standard microfabrication processes. This architecture has potential applications in heliocentric, geocentric, and three-body orbits.

\footnotetext{
* Reproduced with permission from Acta Astronautica.
}

Originally published in Vol. 67, 2010, pp. 108-121. 


\subsection{List of Symbols}

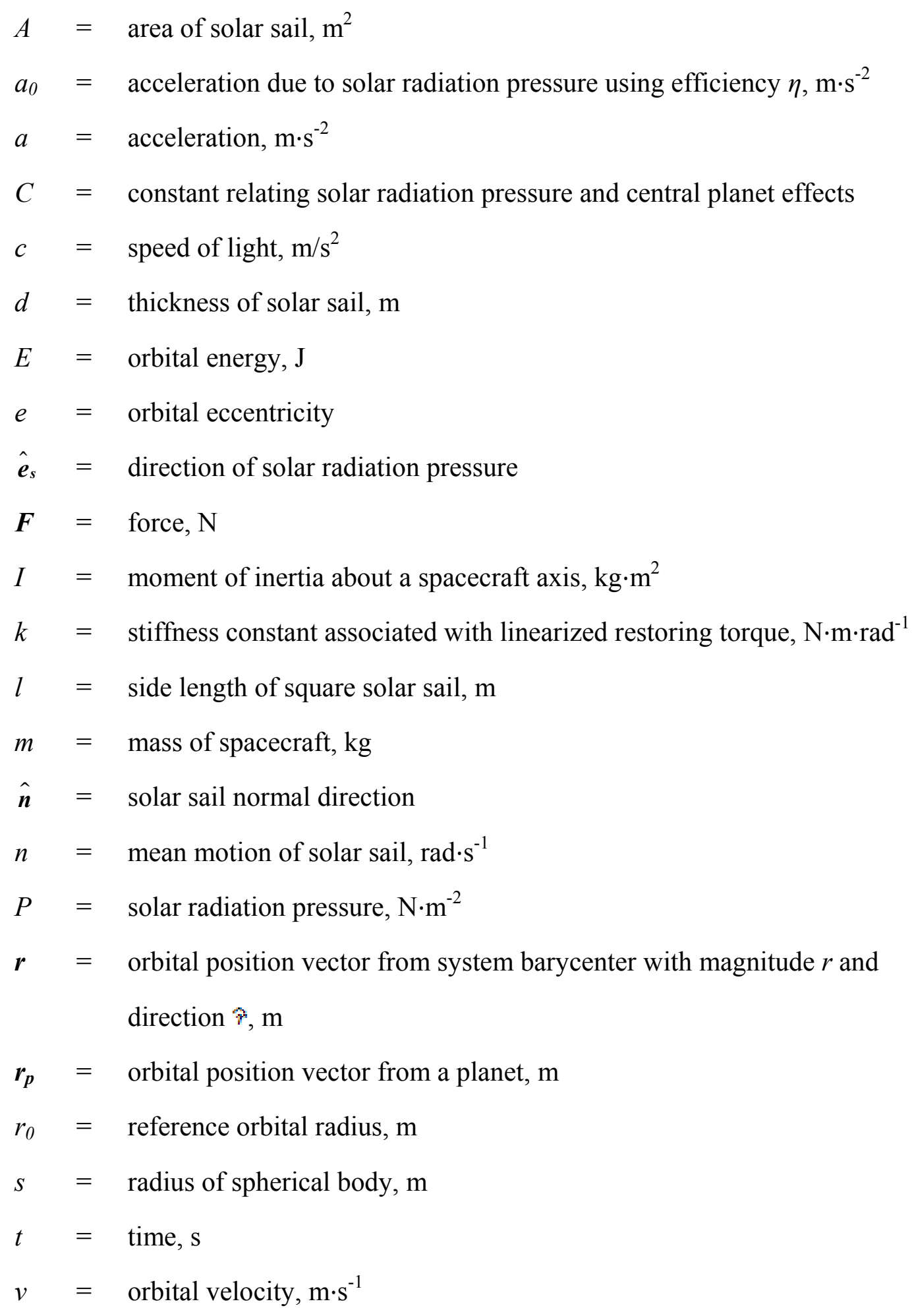




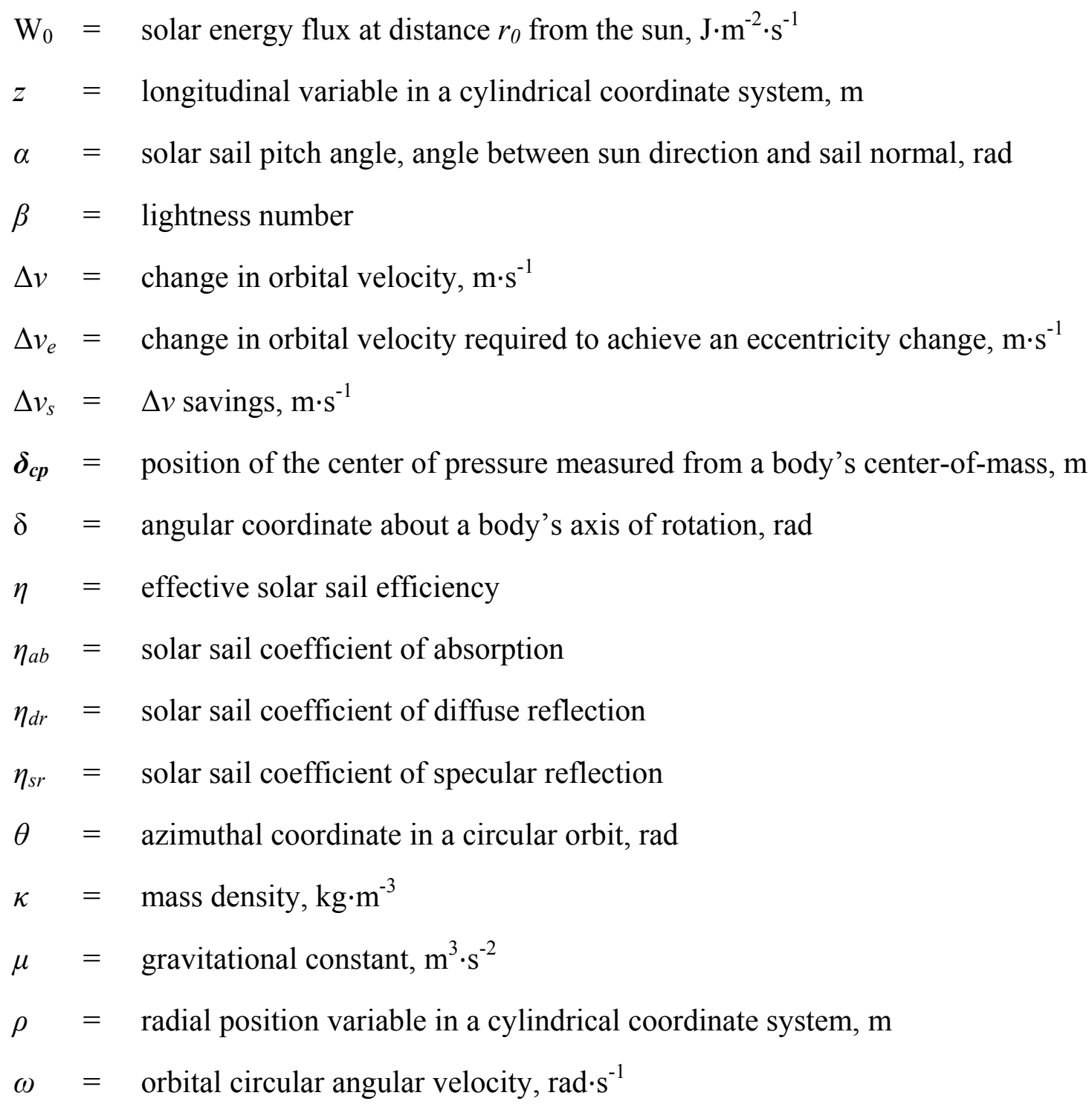

\section{Subscripts}

$G=$ gravitational

$S R P=$ body acted on by solar radiation pressure 


\subsection{Introduction}

Dust particles in space vary in size from a few molecules to $100 \mu \mathrm{m}$ and have a mass smaller than a few $\mu \mathrm{g}$. At these length scales, the dust's orbit is significantly affected by many orbital perturbations that are typically irrelevant in spacecraft trajectories. Length scale influences the relative importance of these effects through a combination of area- or length-dependent force and volume-dependent mass, for a more-or-less fixed material density. These accelerations can compete with gravity to produce trajectories sufficiently different from their Keplerian analogues to serve as new mission opportunities. For example, solar radiation pressure (SRP) ejects dust from the solar system; electromagnetic effects capture and eject dust in planetcentered orbits; and aerodynamic drag captures and lands dust without the hightemperature ablation that larger meteors typically experience. This research is aimed at harnessing these natural, small-body dynamics in order to enable new propulsion techniques and missions based on them.

In pursuit of this goal, we are working to develop a self-contained spacecraft bus dubbed "sprite", whose length scale enables it to demonstrate useful propellantless propulsion. Inspired by the 1957 Sputnik mission, we focus on a simple, passive, mission design that achieves Sputnik's rudimentary demonstration of spaceflight capability. For three weeks, the 23 inch diameter Sputnik I broadcasted its internal temperature and pressure as it orbited. A half century later, we anticipate duplicating Sputnik's achievement using less than one ten-millionth of its mass. This design rethinks and integrates the traditional subsystems (power, propulsion, communications, etc) into a single silicon microchip spacecraft capable of unlimited $\Delta v$. The sprite bus is a $25 \mu \mathrm{m}$ thick, $1 \mathrm{~cm}$ square, $7.5 \mathrm{mg}$ silicon microchip with integrated solar cells and signal-transmitting circuitry, as illustrated in Figure 3.1 and 
described in Section V. The spacecraft is passive in that it has no sensor feedback or feedback-based actuation. In place of traditional thrusters and attitude-control actuators, it exploits small-body dynamics to accomplish maneuvering.

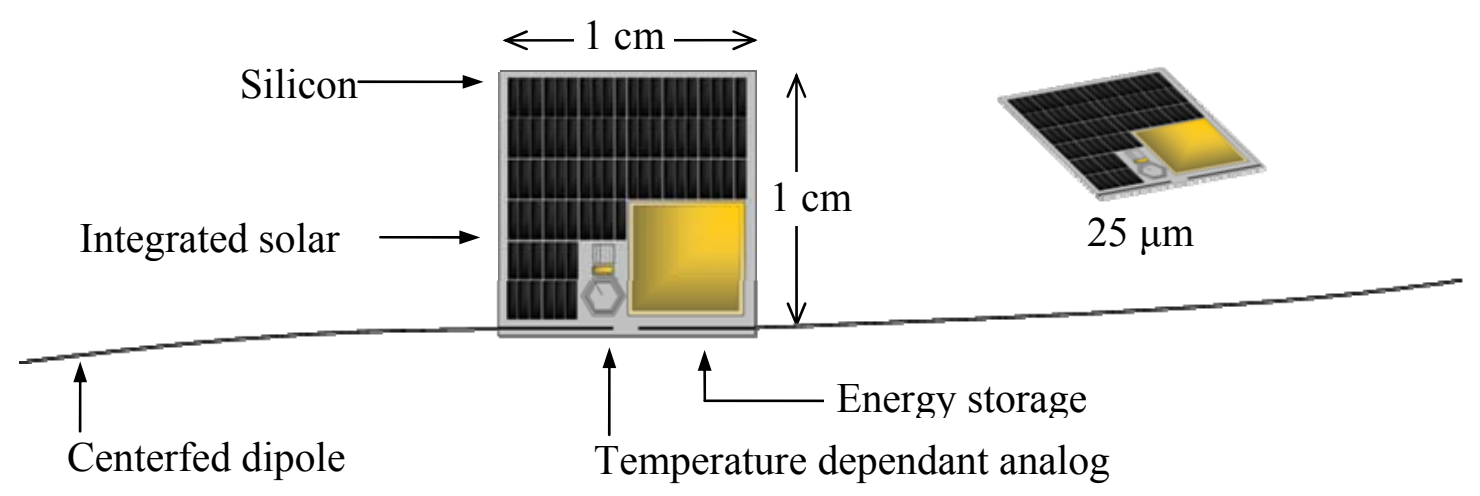

Figure 3.1. Sprite bus layout

Several other groups are actively and successfully developing monolithic integrated-circuit (IC) silicon spacecraft (sometimes called a "spacecraft-on-a-chip"), notably the Surrey Space Centre [1,2], the Aerospace Corporation $[3,4,5]$, and the Jet Propulsion Laboratory[6]. Barnhart [1] provides an historical summary of these efforts. The primary incentives for these research programs are persuasive: economies of production, reduced launch mass, and distributed sensing opportunities. However, these objectives generally differ from the primary goal of this work: capitalizing on length scaling to achieve feasible orbit control. These programs envision spacecraft with masses on the order of grams, which are certainly unusually small by any traditional standard but are still thousands of times larger than our target.

This paper focuses on the efficacy of SRP as a means of propellantless propulsion for a millimeter-scale spacecraft. In this context, the millimeter-scale spacecraft is a solar sail [7]. SRP can greatly influence, if not dominate, the orbital behavior of dust. 
For example, dust detectors on Pioneers 8 and 9 discovered streams of dust on outbound hyperbolic orbits. These streams originate from comets, which shed the dust throughout their orbit. When released near the sun, some of this dust experiences a sudden change in acceleration due to solar radiation pressure that sends them on escape trajectories $[8,9,10]$.

Solar-sail scale has implications not only for performance but also in hardware design and implementation. Typical sail designs are extremely large and combine physical principals that span many orders of magnitude. Greschik [11] suggests that dimensional challenges are primarily responsible for the as yet unsuccessful solar-sail tests, despite thirty years of attempts. The range of magnitudes involved in solar sails make structural analyses intractable, fabrication demanding, and ground testing nearly impossible. These challenges have motivated some to propose and develop so-called solar kites [12], microsolar sails [13], and nanosails that minimize some of these scaling challenges. In August 2008, NASA's NanoSail-D would have been the first spacecraft to demonstrate solar sailing, were it not for the launch-vehicle failure. Like these small solar sail concepts, the millimeter-scale design avoids many of these dimensional issues. It can be fabricated using integrated-circuit techniques and can be readily tested in a $1 \mathrm{G}$ environment. However, the millimeter scale offers additional benefits. As this paper argues, it enables more subtle passive behaviors and seeks to capitalize on natural dynamics, avoiding the nontrivial challenges associated with solar-sail control and actuation.

McInnes [8] recognizes the promise of propellantless spacecraft designs that approach centimeter and millimeter scales. He envisions smart, compact sails that use microelectromechanical systems (MEMS) to navigate the solar system autonomously. We pursue that shared vision here by surveying a variety of solar sail maneuvers using 
passive and feasible attitude control methods and by addressing design and fabrication progress for a candidate spacecraft.

\subsection{Solar Radiation Pressure}

SRP results when sunlight strikes a surface. A photons carries with it energy and momentum related to its wavelength. When a photon is absorbed or reflected by a body, momentum is exchanged. This momentum exchange can have a significant, measurable effect on the attitude and orbit of a body illuminated by the sun.

\subsubsection{SRP Acceleration}

The pressure $P$ from the sun due to this photon momentum transfer is

$$
P=\frac{W_{0}}{c}\left(\frac{r_{0}}{r}\right)^{2} \hat{\boldsymbol{r}},
$$

where $c$ is the speed of light, $W_{0}$ is the energy flux from the sun taken at a distance $r_{0}$ from the sun, and $\hat{r}$ is the direction of the position vector $\boldsymbol{r}$ with magnitude $r$. The force $\boldsymbol{F}_{\boldsymbol{S R} \boldsymbol{P}}$ due to this pressure acting on an opaque body depends on the surface characteristics, which determine how incoming light is specularly reflected, diffusely reflected, or absorbed. The dimensionless constants $\eta_{s r}, \eta_{d r}$, and $\eta_{a b}$ account for each of these respective effects:

$$
\begin{gathered}
\boldsymbol{F}_{\boldsymbol{S R P}}=P A \cos \alpha\left[\left(2 \eta_{s r} \cos \alpha+\frac{2}{3} \eta_{d r}\right) \hat{\boldsymbol{n}}+\left(\eta_{a b}+\eta_{d r}\right) \hat{\boldsymbol{e}}_{S}\right] \\
\eta_{s r}+\eta_{d r}+\eta_{a b}=1
\end{gathered}
$$


where $A$ is the area of the body exposed to photons when facing sunward, $\alpha$ is the sail pitch angle defined by the body's surface-normal unit vector $\hat{\boldsymbol{n}}$ and the direction $\hat{\boldsymbol{e}}_{s}$ of the incoming photons [14]. The acceleration is then

$$
\boldsymbol{a}_{S R P}=\frac{\boldsymbol{F}_{S R P}}{m}
$$

a term that is proportional to the ratio of the body's area to mass $m$. A simplified scalar acceleration $a_{0}$ is useful when comparing solar sail designs

$$
a_{0}=2 \eta P \frac{A}{m} \text {. }
$$

This scalar result is appropriate only for a case in which the sail is facing the sun at a distance of $r_{0}$. Here, the more precise model that is based on the coefficients $\eta_{s r}, \eta_{d r}$, and $\eta_{a b}$ is replaced by a single efficiency $\eta$, henceforth taken to be 0.85 [8]. The ratio of this simplified acceleration to the acceleration due to solar gravity, $a_{G}$, is the lightness number $\beta$

$$
\beta=\frac{a_{0}}{a_{G}}
$$

A body's $A / m$ is related to its geometry. In the case of a sphere, a common model for interplanetary dust $[15,16]$, the ratio $A / m$ varies inversely with the body's radius $s$ and mass density $\kappa$

$$
\frac{A}{m}=\frac{\pi s^{2}}{\frac{4}{3} \pi \kappa s^{3}}=\frac{3}{4} \frac{1}{\kappa s}
$$

The acceleration due to SRP is inversely proportional to characteristic length. This ratio accurately describes the acceleration's dependence on body size for lengths above the wavelength of the sun's spectrum. At length scales below the wavelength of 
visible light, the particles are simply too small to absorb or scatter the photons. For most materials exposed to sunlight, the critical size is a tenth of a micron [10]. Highly reflective particles of this size can achieve $\beta>1$ and escape solar gravity.

Treating characteristic size as a design parameter, we seek to minimize the size of the sprite spacecraft and replicate the behavior demonstrated by small dust particles. Like most solar sails, the sprite design resembles a flat plate. For such a shape, with its maximum area normal to the sun, $A / m$ simplifies to

$$
\frac{A}{m}=\frac{l^{2}}{\kappa l^{2} d}=\frac{1}{\kappa d},
$$

where $l$ is the plate's side length and $d$ is the plate's thickness. Reducing the thickness of the plate or uniformly scaling down all lengths increases the SRP acceleration. This observation motivates the use of ultra-thin $25 \mu \mathrm{m}$ thick silicon, the thinnest conceivable substrate for an IC. With this thickness, the candidate architecture achieves $a_{0}=0.10 \mathrm{~mm} / \mathrm{s}$ and $\beta=0.0175$. To achieve $\beta>1$, the thickness would need to be reduced to $0.3 \mu \mathrm{m}$. Figure 3.2 relates sample values of area and mass for dust and a selection of typical solar sail and nanosail designs. Diagonal lines of constant $A / m$ illustrate that all solar sail designs fall within a comparable $A / m$ ratio. The two designs, described throughout this work, are located along these same contours, suggesting that this approach achieves comparable performance to traditional designs. 


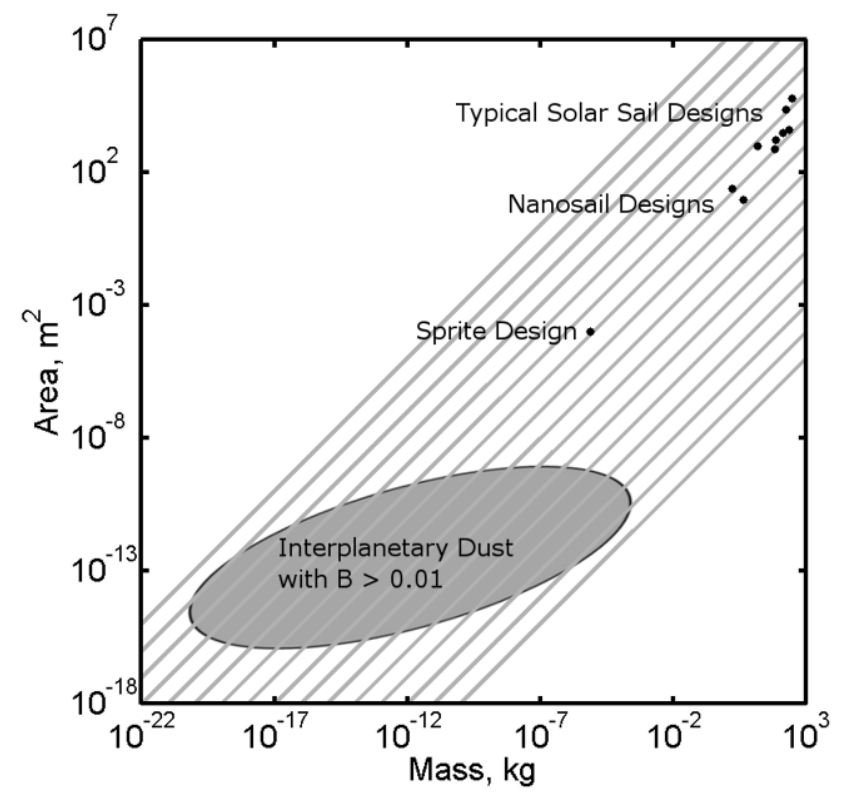

Figure 3.2. Sample values of exposed area to mass for dust and solar sails. Diagonal lines show constant $A / m$ contours $[8,10,14,17]$.

As bodies absorb solar energy, they experience the so-called Poynting-Robertson drag $[17,10]$. This drag is associated with a difference in the relativistic Doppler shift between the energy received forwards and backwards with respect to the body's motion. For dust on bounded heliocentric orbits, this weak drag determines the longterm orbital evolution and eventually collapses these orbits into the sun after thousands of years. The Yarkovsky force is associated with a similar effect: differential thermal radiation of rotating bodies [5]. These effects are weak, even for dust, so we neglect them in this study. 


\subsubsection{Coupled Attitude and Orbital Dynamics}

SRP accelerations are a function of the orientation of the surface normal vector. Thus the orbital and attitude dynamics of solar sails are coupled in general. The surface of dust is often modeled as homogeneous and spherical for the purposes of calculating $\beta$ and establishing a normal vector [10]. In this case the normal vector is taken to be constantly sun-pointing. This particular geometry decouples the attitude dynamics from the orbital dynamics [18].

Observations of the mass distribution of dust in the solar system lead some to believe that most dust is spinning very rapidly. Particles that spin rapidly enough may disintegrate via rotational bursting [10]. The spin is thought to be generated by "windmill" [19] or "paddlewheel" [20] torques that result from SRP acting on dust's anisotropic surface. These spin rates suggest that the dust particles have nonnegligible angular momenta and therefore manifest attitude stiffness. Rather than chaotically tumbling throughout their orbit, the particles may be rapidly spinning with inertially fixed angular momentum vectors. There may be passive dynamical applications for a spinning solar sail, though this paper does not pursue them.

Planning a solar-sail maneuver requires a stable and known heading in the presence of disturbance torques such as free molecular flow, magnetic fields, and gravitygradient effects. For a plate geometry, however, SRP induced torques likely dominate these effects. An SRP disturbance torque $\tau$ is produced if the center of pressure $\boldsymbol{\delta}_{c p}$, where the effective SRP force $\boldsymbol{F}$ acts, does not coincide with the spacecraft's center of mass [21]

$$
\boldsymbol{\tau}=\delta_{c p} \times \boldsymbol{F}_{S R P} .
$$


This torque vanishes only when $\delta_{c p} \| \boldsymbol{F}_{S R P}$ or $\delta_{c p}=\boldsymbol{0}$. The first case represents a marginally stable equilibrium heading where the center of pressure is located along the line between the sun and the center of mass. In this orientation, the sail is said to be statically balanced $[22,22]$. The second case represents an unstable equilibrium that is not practically realizable. An arbitrarily small magnitude of $\boldsymbol{\delta}_{c p}$ results in a non-zero torque until the spacecraft reorients and becomes statically balanced. Careful design can minimize the offset, but the required precision is nontrivial. Errors on the order of $100 \mu \mathrm{m}$ can result in a near $90^{\circ}$ "edge-on" equilibrium pitch angle. Therefore, with no access to SRP or solar cell power, this heading is undesirable. Any architecture must therefore account for the likelihood of this disturbance torque.

In the following analysis, we consider an attitude that replicates dust dynamics. We develop methods for achieving a passive sun-pointing attitude. This decouples the attitude and orbital dynamics and offers maximum solar-cell power. The attitude also maximizes the magnitude of SRP by fixing $\alpha=0$. However, the direction of $\boldsymbol{a}_{\boldsymbol{S R \boldsymbol { P }}}$ lies always along the sun line, limiting its use.

\subsection{Applications}

This section evaluates orbit opportunities for the given architecture: a passive, sunpointing solar sail. With these restrictions, the effects of SRP are considered for heliocentric, geocentric, and three-body orbits.

\subsubsection{Two-Body Heliocentric Orbits}

The Infrared Astronomical Satellite (IRAS) observed several groupings of dust clearly originating from comets. These groupings follow elliptical heliocentric orbits with non-Keperian parameters which can be explained by SRP $[23,24,25]$. In a 
heliocentric orbit, the sun line is parallel to the orbit-radial direction, $\hat{\boldsymbol{r}} \| \hat{\boldsymbol{e}}_{\boldsymbol{s}}$. In this orientation, SRP effectively reduces the heliocentric gravitational constant $\mu$ by the fraction $\beta$, which yields a modified two-body equation of motion [26]

$$
\ddot{\boldsymbol{r}}=-\frac{\mu_{s}(1-\beta)}{r^{2}} \hat{\boldsymbol{r}} \text {. }
$$

For $\beta<1$, this equation produces conic-section orbits with increased orbital energies, as IRAS observed.

As dust particles are released, SRP immediately alters their orbits. While it is still part of the comet, the particle's orbit is negligibly affected by SRP. After release, the particle's new energy $E_{S R P}$ is increased

$$
E_{S R P}=\frac{v^{2}}{2}-\frac{\mu_{s}(1-\beta)}{r} .
$$

If $E_{S R P}$ is positive, the dust escapes on a hyperbolic orbit [9,10,11]. Streams of these so-called $\beta$-meteoroids were detected by both the Galileo [27] and Ulysses [28] missions. In this application, SRP can be thought of as adding an effective $\Delta v$ to the particle. For a particle released at perihelion, the required $\beta$ for escape is based on the initial eccentricity $e$ prior to ejection [10]

$$
\beta \geq \frac{(1-e)}{2}
$$

One can imagine a similar maneuver for a sun-pointing solar sail. Taking a circular heliocentric orbit at radius $r_{0}$, the minimum impulsive $\Delta v_{e}$ required for a spacecraft to achieve a given eccentricity is

$$
\Delta v_{e}=\sqrt{\frac{\mu_{s}}{r_{0}}}(\sqrt{1+e}-1)
$$


The solar sail escape therefore offers a $\Delta v$ savings of

$$
\Delta v_{\text {saving }}=\sqrt{\frac{2 \mu}{r_{0}}}(1-\sqrt{1-\beta})
$$

For the proposed sprite design at $1 \mathrm{AU}$, Eq. 14 predicts a modest savings of $0.37 \mathrm{~km} / \mathrm{s}$, only $3 \%$ of the direct escape requirement.

Alternative applications for the modified two-body heliocentric equation of motion have been proposed by the solar-sail community. For example, novel satellite formations can be produced using the modified angular velocity $\omega$ of a sun-pointing solar sail in a circular orbit

$$
\omega_{S R P}=\sqrt{\frac{\mu_{s}(1-\beta)}{r^{3}}} .
$$

The radially directed SRP allows two satellites to orbit with the same angular velocity at unique radii [8]. Alternatively, two spacecraft can orbit at the same radius, but with unique angular velocities, perhaps to spread out azimuthally along an orbit. In the first application, a sprite spacecraft at 1 AU could orbit at a radial distance of $877,000 \mathrm{~km}$ from a spacecraft that experiences negligible SRP effects, such as a point-mass satellite. In the second application, a sprite spacecraft at $1 \mathrm{AU}$ could be induced to drift at a rate of 31 arcseconds per day (1.3 million $\mathrm{km}$ per day) along track from a point-mass spacecraft. 


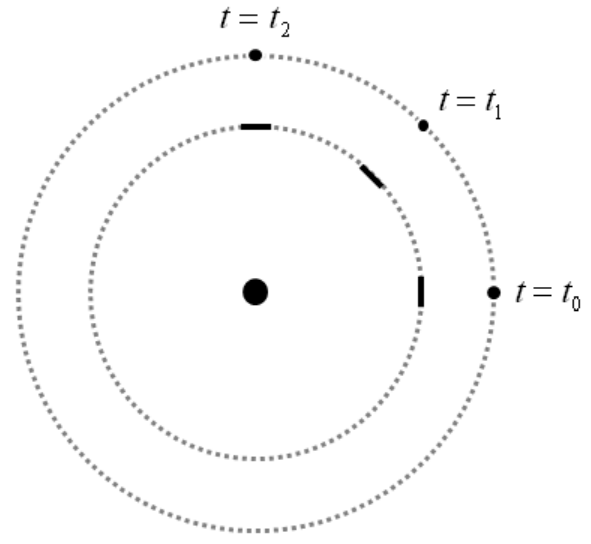

a.)

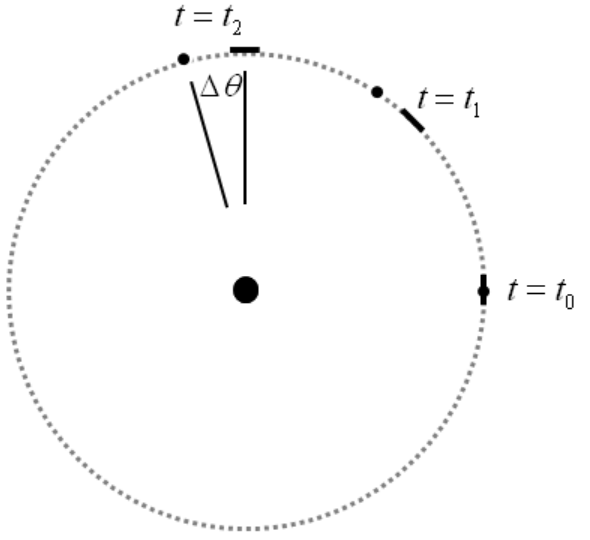

b.)

Figure 3.3. Simple circular maneuvers enabled by constant sun-pointing attitudes: a.) constant anomaly formation and b.) along-track separation

Passive halo or displaced formations are attractive and have been the subject of research $[29,30,31]$ but require a non-sun-pointing attitude. Here, the sail follows a zero-inclination orbit that is displaced from the ecliptic. McInnes [30] finds that the required pitch angle is a function of the vertical displacement $z$, the planar radius $\rho$, the modified angular velocity $\omega$, and the circular Keplerian angular velocity $\omega=\sqrt{\mu / r^{3}}$

$$
\tan \alpha=\frac{\left(\frac{z}{\rho}\right)\left(\frac{\omega_{S R P}}{\omega}\right)^{2}}{\left(\frac{z}{\rho}\right)^{2}+\left[1-\left(\frac{\omega_{S R P}}{\omega}\right)^{2}\right]} .
$$

This term can be made small but is zero (i.e. sun-pointing) only in the impractical or non-displaced cases of $z, \rho$, or $\omega_{S R P}$ set to zero. The only out-of-plane motion a sun- 
pointing solar sail experiences must be associated with inclination. This result is intuitive - a reduction in the magnitude of an attractive $r^{-2}$ field does not affect the morphology of the available orbits.

\subsubsection{Two-Body Geocentric Orbits}

The effects of SRP on dust in planetary orbits have been well researched, often in conjunction with the rings of Saturn and Jupiter [10,32,33]. SRP is the dominant perturbation at the outer rings, where magnetic fields and tidal forces are weak [34]. Long-term orbit evolution has been evaluated with orbit-averaged perturbation methods that describe the effects of planetary motion about the sun by averaging short term orbit-period effects. After neglecting shadow effects, the orbit-averaged perturbation equations show that semimajor axis is constant and that the remaining elements vary synchronously with the planet's period (see for example, Ref [10], Eqs 34-38). The change in inclination is proportional to inclination $\Delta i \propto-i$, so equatorial orbits are in a stable equilibrium. These effects were first observed on the Echo I [35] and Vanguard I [36] satellites. Echo I was a communications satellite consisting primarily of a reflective $100 \mathrm{ft}$ diameter mylar balloon [37] $\left(A / m \approx 0.1 \mathrm{~kg} / \mathrm{m}^{2}\right)$. It demonstrated large-amplitude periodic eccentricity changes, resulting in a perigee altitude that varied annually between 1500 and $930 \mathrm{~km}$ [38]. Van der Ha and Modi were the first to consider sun-pointing solar sail maneuvers in a geocentric orbit. They demonstrated secular changes in semimajor axis and semilatus rectum using simple bang-bang control laws [39]. A variety of Earth escape maneuvers have since been analyzed that suppose a solar sail with full attitude control [8].

The prospect of SRP induced periodic change in orbital elements suggests applications for distributed sensing. It is as though a single spacecraft can take 
measurements from a multitude of traditional orbits. The periodic behavior of inclination for example, enables a spacecraft to sample throughout a range of inclinations annually. For multiple spacecraft, these variations also present a means of gradual propellantless separation.

Hamilton and Krivov [35] offer a special solution for the periodic motion of planetary dust. This solution describes an equatorial, constant-eccentricity orbit whose longitude of pericenter rotates with the planet-sun line (i.e. the periapsis is sunpointing). This unique orbit is thought to have been identified in Saturn's "charming ringlet" [40]. The orbit's eccentricity is related to lightness number

$$
\begin{gathered}
e=\frac{C}{\sqrt{1+C^{2}}}, \\
C=\frac{3}{2} \frac{n_{S R P}}{n_{p}} \frac{a_{S R P}}{a_{G P}},
\end{gathered}
$$

where $n_{p}$ and $n$ are the mean motions of the central planet and spacecraft respectively, and $a_{G P}$ is the magnitude of the planet's two-body gravitational acceleration. Figure 3.4 and Figure 3.5 show the numerical integration of a sample orbit at Earth for $\beta=$ 0.0175 in both the planet-fixed and sun-planet fixed frames. These figures illustrate two potential applications. In the planet-fixed frame, the spacecraft sweeps through an annulus of space over the course of a year. In this simulation, the apogee of the spacecraft corresponds to geostationary orbit (GEO), so that the spacecraft encounters most or all GEO orbital slots as it is annually precessed. In the sun-planet fixed frame, the orbit stays aligned with Earth's magnetotail. This orbit may offer scientific sensing applications or applications related to communications satellites. 


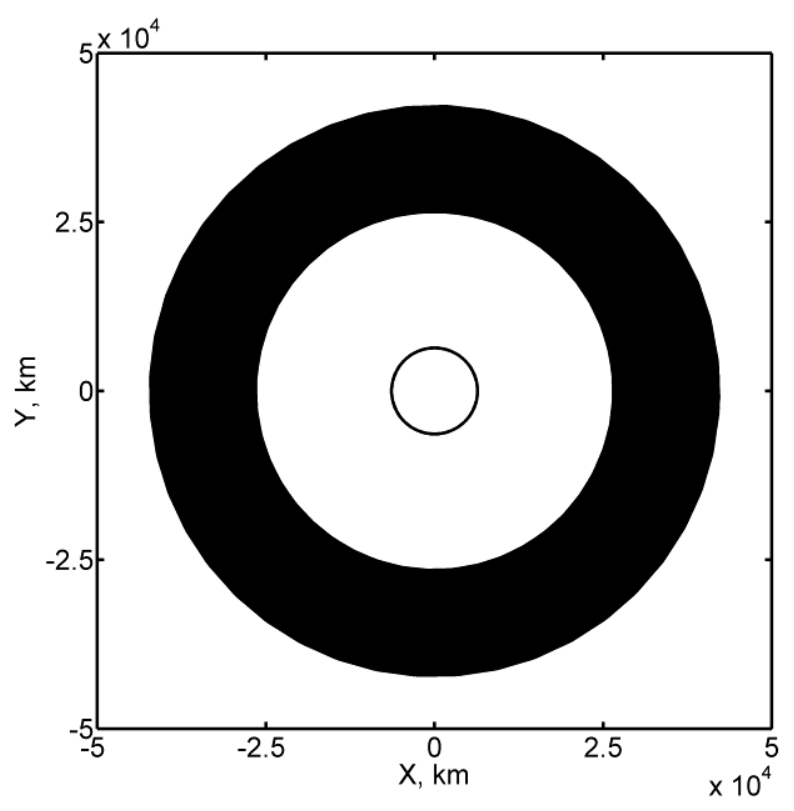

Figure 3.4. Earth fixed time history of a geocentric spacecraft with SRP over oneyear.

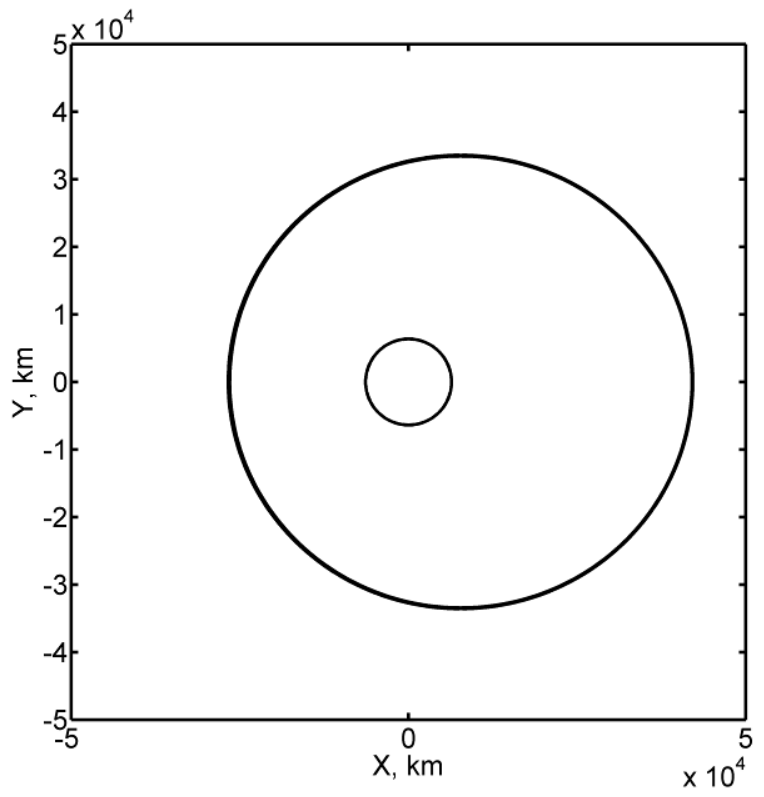

Figure 3.5. Sun-Earth fixed time history of a geocentric spacecraft with SRP over one year. The sun (not shown) is located along the $-\mathrm{X}$ axis. 


\subsubsection{Three-Body Orbits}

SRP acting on the orbits of dust in three-body orbits is thought to be observed in the interplanetary dust complex. This is a cloud of dust centered on the ecliptic that causes zodiacal light by bending the sun's light back towards Earth [41]. Some of the asymmetries in this cloud are associated with SRP, which groups the dust in regions near modified Lagrange points [42] and in planetary wakes [42,43]. This section evaluates these dynamics and offers potential applications for a spacecraft orbiting in the Sun-Earth system.

The five classical Lagrange points $\left(L_{1}-L_{5}\right)$ are shifted for a test particle with radially oriented SRP acceleration with $0<\beta<1$. The addition of $\beta$ causes these equilibria to shift along simple paths $[43,45,8]$. The three collinear points move along towards the sun along the Earth-Sun line. The triangular points follow an arc of constant radius $r_{p}$ towards the sun, as illustrated in Figure 3.6.

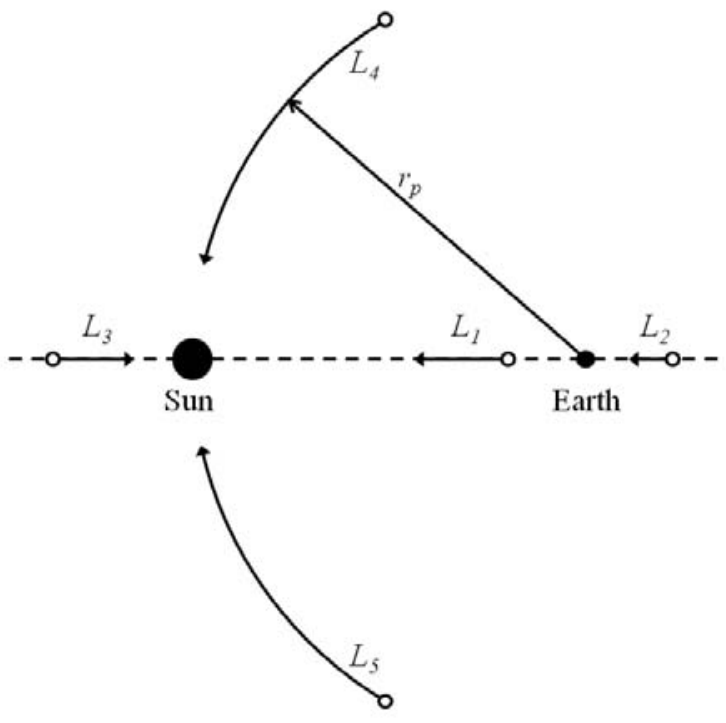

Figure 3.6. Motion of the Lagrange points in a corotating reference frame for a sunpointing spacecraft with increasing $\beta$, $(0 \leq \beta \leq 1)$. [42] 
The Lagrange points are attractive to trajectory designers because they are fixed with respect to the Earth-Sun line. The three collinear points are unstable, so a spacecraft located at those points must expend some energy to maintain its position, albeit a potentially small amount. The triangular points are stable, so bodies (including dust) tend to asymptotically migrate to those locations when nearby. Using a linear analysis, Schuerman [43] shows that the instability of the collinear points is unchanged by the addition of SRP. The triangular points have an added constraint for stability that remains satisfied in the solar system with $\beta<1$.

In solar sail literature, three-body orbital analyses are common, particularly because many applications are suited to low $\beta$ sails. A prominent application uses a solar sail with feedback control to both move and stabilize the collinear $L_{1}$ point for solar observation [44]. The propellantless nature of solar sails ensures a long lifetime in the presence of disturbance accelerations.

The SRP induced motion of the $L_{1}$ point offers applications for solar weather observation. In some cases, the proximity of a spacecraft to the sun is related to the warning time at Earth. For example, coronal mass ejections have characteristic velocities on the order of $500 \mathrm{~km} / \mathrm{s}$ [45]. As illustrated in Figure 3.7, the distance gained at $L_{1}$ using SRP can correspond to a significant increase in the warning time at Earth. The lines correspond to maximum, mean, and minimum warning times using $280 \mathrm{~km} / \mathrm{s}, 470 \mathrm{~km} / \mathrm{s}$, and $1000 \mathrm{~km} / \mathrm{s}$ solar wind speeds [47]. The candidate spacecraft offers approximately 65 minutes of warning time - an improvement of 13 minutes over the mass-only case. With enough sprite spacecraft, the sum of these observations could both predict and depict the wave front of earthbound solar phenomena. Though the spacecraft could not stabilize their location, they may experience sufficiently long lifetimes in unstable quasi-periodic orbits to be useful. 


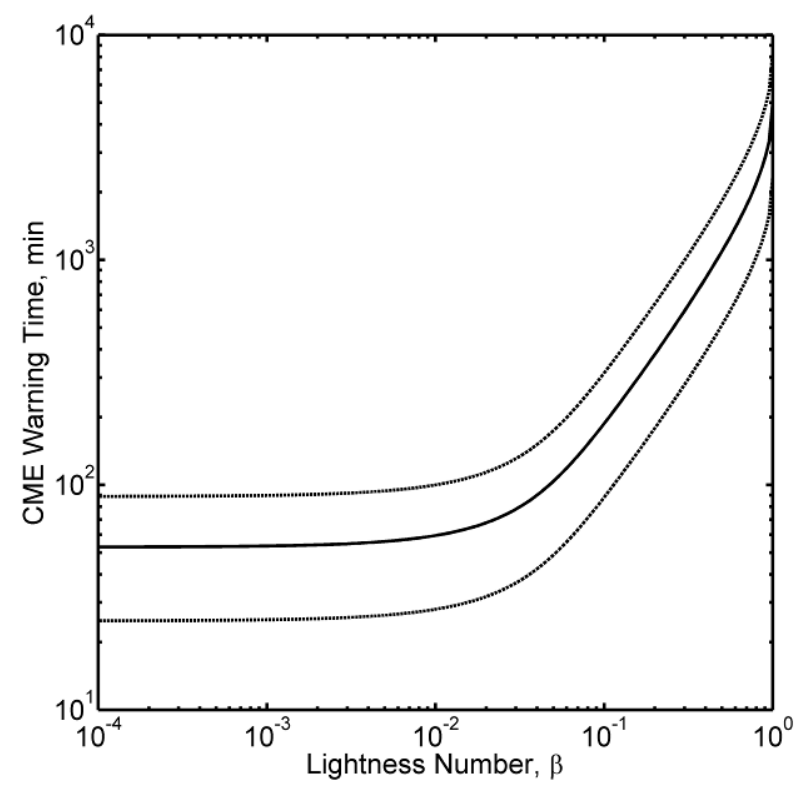

Figure 3.7. Estimated CME warning time from L1 for a spacecraft with increasing $\beta$, $(0 \leq \beta \leq 1)$.

\subsection{Passive Sun-Pointing Attitude Control}

These maneuvers require the design of a suitable attitude architecture that can maintain a sun-pointing heading. As early as 1959, SRP disturbance torques were proposed as a means of attitude actuation [23]. If properly designed, a spacecraft can exploit these torques to produce a stable sun-pointing attitude [46]. Rather than approach this task from a control actuation perspective, we seek geometries that passively align themselves in the presence of SRP. A sphere, for example, decouples the attitude and orbit mechanics in that it experiences only a radial force, regardless of its orientation [19]. Indeed, one of the first solar sail designs was a spherical balloon proposed by Kraft Ehricke $[19,47]$. These designs are simple, but suffer from low $\beta$ and limited access for solar cells. Rather than a sphere, the first design we consider bears a resemblance to a sun-pointing cone. Using three of the plate-like sprite spacecraft, we propose forming the corner of a cube, as shown in Figure 3.8. 
Kirpichnikov et al. [48] and van de Kolk and Flandro [49] have demonstrated that this geometry creates a global, marginally stable (about two of three axes) sun-pointing attitude whose nonradial force components cancel in both 2D and 3D orientations. SRP torques orient the composite body such that the three plates' common corner reaches an equilibrium angle with respect to the sun line. For uniform, equal-length plates contemplated here, the axis from the center of mass to the common corner points directly at the sun. The off-axis components of the SRP force and torques cancel each other, yielding a net force that is directed sunward.

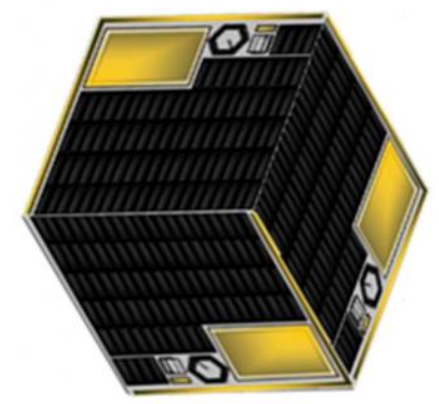

Figure 3.8. Stable, sun-pointing corner-cube architecture.

One may be tempted to reorient the spacecraft such that it forms a concave cornercube retroreflector. This geometry reflects light directly back towards the sun, maximizing the solar pressure's effect for the given area, and cancelling one of the $\cos (\alpha)$ terms in Equation 2. However, this equilibrium orientation is naturally unstable [50].

The disadvantage of the stable architecture is a less effective $A / m$ ratio:

$$
\frac{A}{m}=\frac{\frac{3}{\sqrt{3}} l^{2}}{3 \kappa l^{2} d}=\frac{\sqrt{3}}{3} \frac{1}{\kappa d} .
$$


An architecture that uses three of the candidate sprite spacecraft yields a lightness number of 0.010 . This architecture has the added benefits of redundancy and increased antenna transmission coverage. Some differentiation among the chips may also improve functionality.

A similar concept uses the orientation of surface elements or "facets" to stabilize a sun-pointing plate spacecraft $[50,51,52]$. Each facet produces a torque according to its orientation and surface coating. By strategically placing these facets, the net optical properties can be made to vary with pitch angle. Harris and Wehner designed a radially symmetric pattern of such facets using primary reflection and absorption models. This demonstrated that locally stable orientations can be achieved by coating the faces of half of the facets with either reflective or absorptive material. A rectangular example is shown in Figure 3.9. The surface is symmetric when viewed from the top and asymmetric when viewed from other angles. When placed in an initially sun-pointing attitude, it will tend maintain that heading in spite of disturbances.
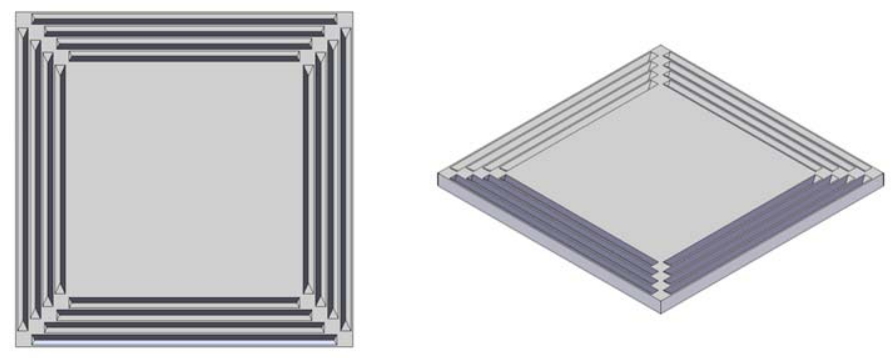

Figure 3.9. Faceted surface viewed from top and isometric viewpoints.

Earlier treatments considered geometries in which primary reflection adequately described the path of impinging light $[53,54]$. In the geometry under consideration here, secondary reflection cannot be neglected. Even at zero pitch angle, light that is 
specularly reflected from one facet impinges on the adjacent facet, causing a secondary force. A ray-tracing code captures these effects. The force on each surface or facet is evaluated in terms of that facet's reflective and absorptive properties, according to Eq. 2. Light that is specularly reflected is propagated to the adjacent facets where this equation is applied a second time. This analysis depends on relatively small pitch angles to avoid the need to represent shadowing effects. In recognition of the likelihood that the surface properties are not ideal, $\eta_{s r}=0.80$ and $\eta_{a b}$ $=0.20$ characterize reflective surfaces, and $\eta_{a b}=1.0$ absorptive surfaces.

The geometry of the spacecraft in this model resembles the spacecraft shown in Figure 3.9. The outer millimeter that borders the sail's surface is etched with 32 and $6410 \mu \mathrm{m}$ deep trenches with $35^{\circ}$ and $54^{\circ}$ side walls respectively. These angles are dictated by the fabrication method described below. The inner walls are coated to be absorptive, while the outer walls are reflective. Figure 3.10 shows the resultant torque about both body aligned axes as the pitch angle is varied. The results indicate that the facets produce a net restoring torque around the sun-pointing equilibrium.

Figure 3.11 shows the simulated attitude time history for the spacecraft that begins with a non-equilibrium heading. The numerical results suggest that there is negligible loss of net SRP force due to the surface facets. Therefore the effective lightness number remains constant. However, this simulation illustrates two limitations that affect both of these architectures. First, the sun-pointing equilibrium is only marginally stable [23]. Any perturbation tends to produce oscillations about the sun line, as seen in the $\mathrm{x}$ and $\mathrm{y}$ axes of Figure 3.11. These oscillations have a period that can be estimated with a small-angle approximation. The linearized equation of motion about each axis's equilibrium position is

$$
I \ddot{\delta}+k \delta=0
$$


where $I$ is the moment of inertia about the axis and $k$ is a stiffness constant responsible for a linear restoring torque. The familiar natural frequencies for this second order system are the square roots of $I / k$. For a numerically predicted value of $k$ $=1.76 \times 10^{-13} \mathrm{Nm} / \mathrm{rad}$, we calculate and observe a simulated oscillation period of 120 seconds. Added structural damping would eliminate these oscillations, perhaps in the form of flexible MEMS beams or thin wire antennas. The second challenge of this architecture is that the attitude motion about the major axis of inertia, here parallel to the sun line, is unregulated, This behavior is evident in the z-axis plot of Figure 3.11. Though the magnitude of the pitch angle may be fixed, the orientation of the transverse axes about the sun line is free. A force cannot produce a torque along its direction; so, there is no straightforward architecture that causes SRP to generate a restoring torque about the sun line. Gravity gradient cannot be used to constrain this remaining degree of freedom, since it too is radial. Likewise, any active control system requires a sensor input observing a direction other than the sun line. This deficiency prohibits energy-change maneuvers that use a fixed pitch angle, such as simple spiral trajectories [51]. It is possible to use a facetted surface to produce spinup "windmill" torques about the z-axis. However, with low damping, these torques could ultimately destroy the spacecraft via rotational bursting, as is posited for dust particles. 


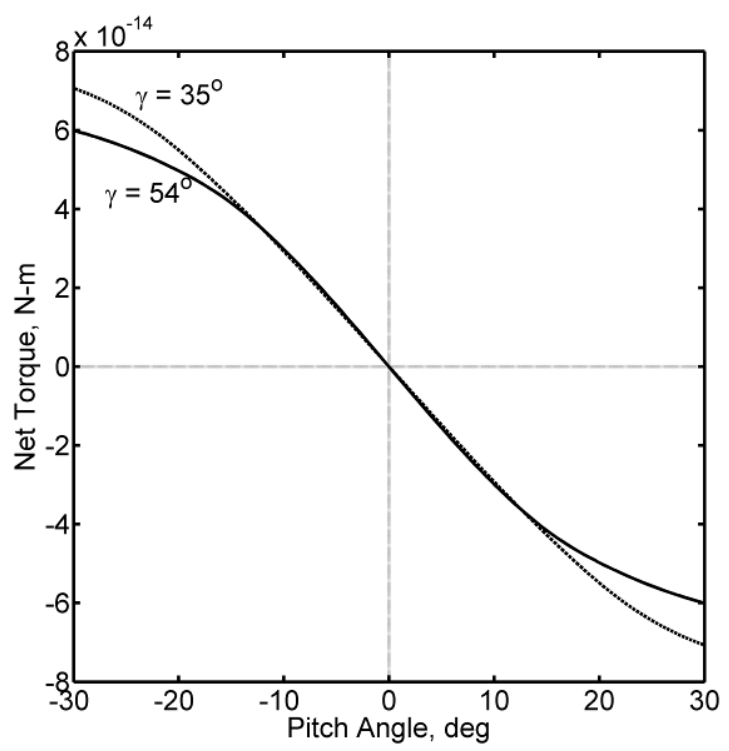

Figure 3.10. SRP induced torque as a function of pitch angle for a faceted surface.
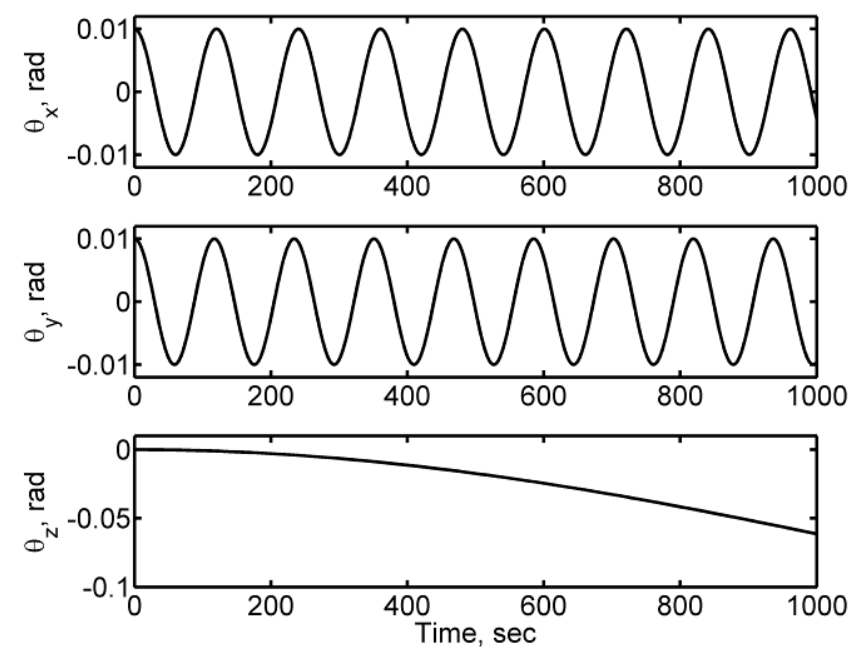

Figure 3.11. Attitude time-history for facetted surface with an initial deviation from the sun line.

A surface profile like this one can be fabricated at the microscale with common microfabrication techniques. One straightforward process uses $\mathrm{KOH}$ to chemically etch out material along the crystal faces of the silicon substrate. This process produces consistent $54.48^{\circ}$ triangular cuts into the exposed surface of a $<100>$ aligned silicon 
plane [53]. This method can produce features in two orthogonal directions along the wafer's surface as dictated by silicon's crystal structure. The fabrication process is outlined in Figure 3.12, which shows a profile of the substrate through six steps. Working at the Cornell NanoScale Science \& Technology Facility, we demonstrated this process. Figure 3.13 shows a Scanning Electron Microscope (SEM) image of our sample wafer with a set of $10 \mu \mathrm{m}$ wide by $7 \mu \mathrm{m}$ deep trenches. This figure shows the end of a trench, where the triangular three-dimensional structure is most visible. Figure 3.14 shows the set of trenches after depositing slightly oxidized chrome at a $60^{\circ}$ angle from the surface.

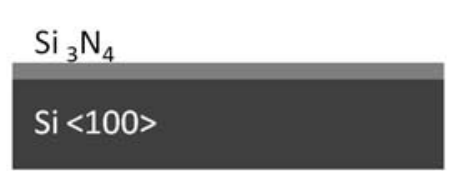

1.) Grow thin layer of silicon nitride over a silicon surface.

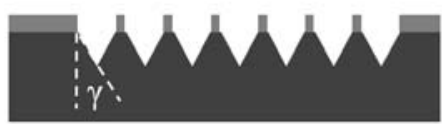

4.) Remove from solution after prescribed time $\gamma=54.48^{\circ}$.

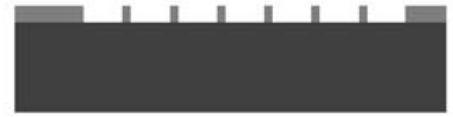

2.) Plasma etch rectangles into the surface of the silicon nitride.

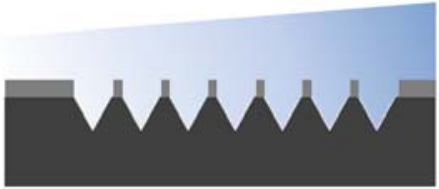

5.) Mask and tilt silicon while spraying or evaporating a secondary coating.

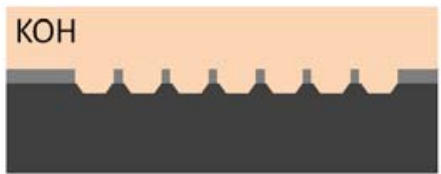

3.) Submerse in buffered potassium hydroxide solution.

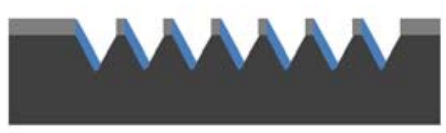

6.) Final surface profile.

Figure 3.12. Anisotropic etching and coating process to produce faceted silicon surface. 


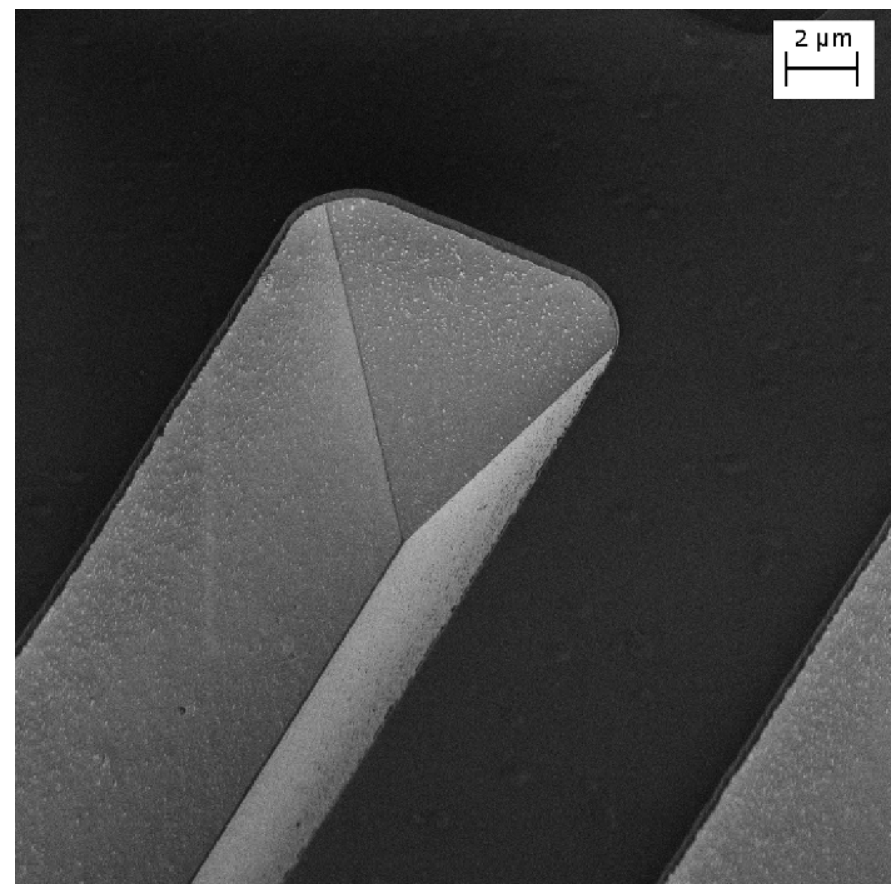

Figure 3.13. SEM image of the end of a sample trench in Si coated with $\mathrm{Si}_{3} \mathrm{~N}_{4}$.

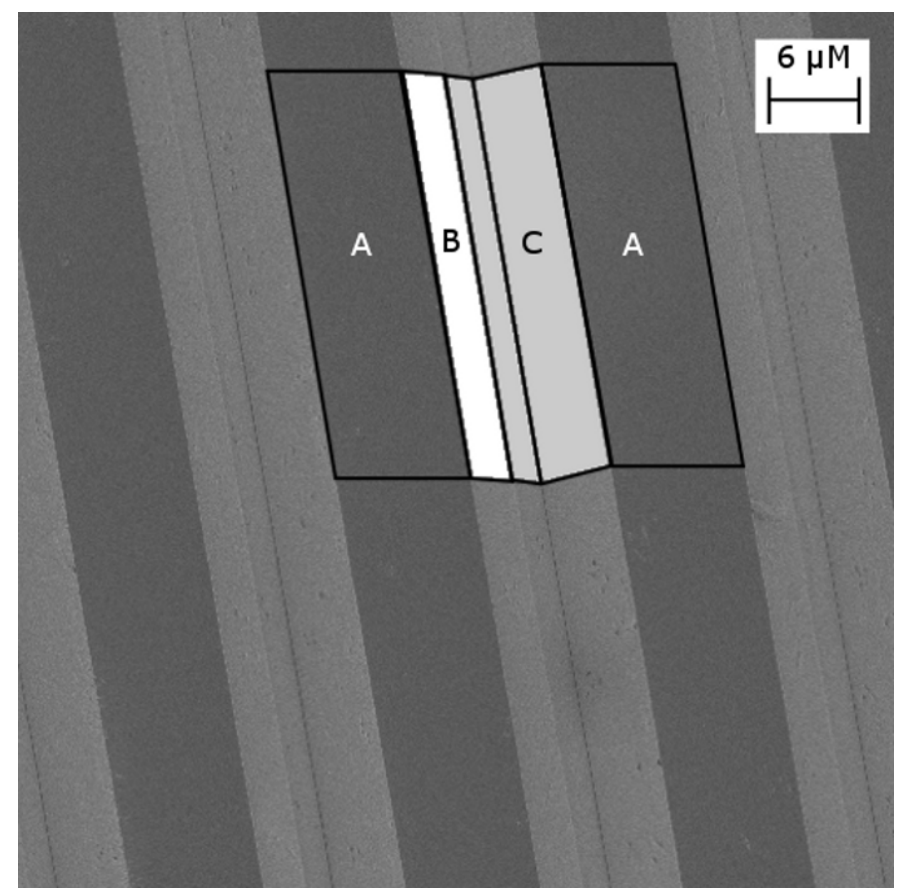

Figure 3.14. SEM image of a $\mathrm{Cr} / \mathrm{Cr}_{2} \mathrm{O}_{3}$ coated sample trench. A.) $\mathrm{Si}_{3} \mathrm{~N}_{4}$ coated with $\mathrm{Cr} / \mathrm{Cr}_{2} \mathrm{O}_{3}$. B.) Si coated with $\mathrm{Cr} / \mathrm{Cr}_{2} \mathrm{O}_{3}$. C.) Bare Si. 


\subsection{Candidate Microscale Infinite-Impulse Spacecraft Bus}

Having motivated length scaling of spacecraft, we now explore how small a

functional spacecraft can be feasibly produced. Others have asked this same question of spacecraft subsystems and have developed "systems-on-chip," which integrate traditionally separate components into a single IC package. The result is a low-cost, light-weight, easily-reproducible chip that accomplishes the same tasks as its conventional, large-scale counterpart. Extrapolation suggests that the next step for this technology is to apply the same techniques to an entire spacecraft to create a "spacecraft-on-a-chip."

We approach this task from a feasibility standpoint, intending to demonstrate functionality at the scales of interest to the dynamic analyses. Thus, we approach the design space with an emphasis on simplicity, achievability, and scalability, rather than with an interest in advancing the state of the art in microfabrication. The goal is to design an extremely small demonstration spacecraft bus, in which we can incorporate the SRP propulsion and attitude control architectures described above. In keeping with spacecraft-design convention, we describe this candidate spacecraft bus in terms of eight traditional subsystems.

\subsubsection{Propulsion}

Traditional propulsion technologies, such as chemical combustion and ion thrust, cannot be easily scaled to the IC level. An exception is so-called "digital propulsion." Lewis et al. have successfully fabricated and demonstrated a device that delivers discrete thrust impulses using micron-sized chambers filled with chemical propellant [54]. Though digital propulsion may prove relevant for this research in the long run, the current research is motivated primarily by propellantless propulsion, in hopes of 
enabling otherwise impossible missions and orbits. SRP, as described above, is among the potential propellantless-propulsion approaches. Future work will include evaluating electromagnetic effects [55] and aerodynamic drag.

\subsubsection{Attitude Determination and Control}

The task of determining and controlling a small spacecraft is perhaps best approached with scaling in mind. Some environmental disturbances may be well suited to producing a known heading. This paper has surveyed methods of achieving locally and globally stable attitude orientations using passive SRP. Torques due to atmospheric drag, outgassing, and magnetism also may prove useful. Future research will also consider active control, perhaps with MEMS as proposed by McInnes [8].

\subsubsection{Structure}

The structure of the spacecraft consists of the volume of semiconductive substrate on which the other subsystems are fabricated. A gallium-arsenide substrate offers improved radiation hardness and the opportunity to produce high-efficiency solar cells. However, the cost of fabricating subsystems on gallium arsenide may discourage its use. We therefore focus on the more common, silicon substrate. At $2300 \mathrm{~kg} / \mathrm{m}^{3}$, solid silicon is significantly denser than $79 \mathrm{~kg} / \mathrm{m}^{3}$, the "rule of thumb" density of a typical spacecraft [56]. Nevertheless, the silicon substrate offers the most near-term opportunity to decrease total mass. As a result, we are exploring methods to work with ultra-thin substrates.

Like a traditional structural subsystem, the substrate must support and mechanically interface the other subsystems, facilitate ground handling, and withstand quasistatic loads during transportation and launch. The sprite design is less concerned with 
vibrations due to resonances with either launch or attitude-control inputs because the natural frequencies of such a structure are far higher than the likely attitude-control bandwidth of any launch vehicle and the sprite itself. Instead, the design is based on the lightest structure on which components can be fabricated: the thinnest possible substrate for a required surface area. For polished silicon wafers, this limit is approximately $200 \mu \mathrm{m}$. Silicon-on-Insulator (SOI) wafers are an alternative. Such wafers consist of an ultra-thin layer of silicon on top of a silicon-oxide layer. This substrate offers structural rigidity and handling during fabrication, after which the silicon-oxide layer can be removed to leave the processed device. Then, arbitrarily thin silicon devices can be produced, although the thickness in this paper is restricted to no less than $25 \mu \mathrm{m}$ for conservatism.

We estimate that sufficient functionality can be achieved in $1 \mathrm{~cm}^{2}$. Of primary interest is the placement of solar cells on both sides of the chip to ensure that power is always available, regardless of attitude. The mass of such a $1 \mathrm{~cm}^{2}$ silicon substrate is $5.75 \mathrm{mg}$. For conservatism, the mass budget includes $30 \%$ margin, yielding a total mass of $7.5 \mathrm{mg}$, which is used in this paper's calculations. The silicon fabrication process consists of additive and subtractive processes, which add or remove material from the substrate to form a device. The net contribution of these processes is assumed to be negligible.

\subsubsection{Communications}

Following Sputnik's example, we conceive the communications subsystem as a transmit-only beacon. The data consists of a single beep at a single frequency - a binary output based on the presence or absence of the carrier. There is no signal per se carried on that frequency. For this simple transmission to be tracked from a ground 
station, it must be powerful enough to overcome free-space loss, atmospheric attenuation, and other noise sources. The communication link's carrier-to-noise-ratio $C / N$ is therefore a useful measure of goodness. This ratio is influenced by the signal's frequency, the orbit's altitude, the transmitter's losses and power, atmospheric conditions, and antenna efficiency. Table 3.1 baselines a downlink communications budget at $433 \mathrm{MHz}$, an amateur satellite band. A single chip can accomplish only so much. A simple way to close the link budget is to select a ground station with very high gain. For example, a $12 \mathrm{~m}$ parabolic dish with a standard figure-of-merit of 0.55 offers $32 \mathrm{~dB}$ of gain at $433 \mathrm{MHz}$ [57]. Using such a ground station, the link budget estimates that a $C / N$ ratio of 10 requires approximately $10 \mathrm{~mW}$ from $500 \mathrm{~km}$. Barnhart [3] notes that it is infeasible to track such a small spacecraft using radar. By contrast, the proposed architecture features a beacon that transmits continuously, and it therefore serves as means for operators to tune the receiver during the first few passes.

Table 3.1. Estimated Communications Link Budget

\begin{tabular}{lcc}
\hline \hline \multicolumn{1}{c}{ Parameter } & Value & Units \\
\hline Frequency & 433 & $\mathrm{Mhz}$ \\
Carrier-to-Noise Ratio & 10 & \\
Altitude & 500 & $\mathrm{~km}$ \\
Free Space Loss & -139 & $\mathrm{~dB}$ \\
Atmospheric Attenuation & -2.5 & $\mathrm{~dB}$ \\
Total Transmitter Attenuation & -2.0 & $\mathrm{~dB}$ \\
Receiver System Noise & 250 & $\mathrm{~K}$ \\
Temperature & & \\
Noise Power & -145 & $\mathrm{dBW}$ \\
Receiver Gain & 32 & $\mathrm{~dB}$ \\
Bandwidth & 1 & $\mathrm{MHz}$ \\
Margin & 2 & $\mathrm{~dB}$ \\
Required Power to Transmitter & 10 & $\mathrm{~mW}$ \\
\hline \hline
\end{tabular}


The carrier frequency would likely be generated by a crystal oscillator. The frequency stability of crystal oscillators depends on temperature. This dependence can be characterized and used to infer temperature from the beacon's center frequency. The carrier signal would then be impedance matched to a center-fed half-wave dipole antenna. This antenna consists of two very thin $17 \mathrm{~cm}$ stiff filaments radiating from the chip. The chief advantage of this antenna is excellent performance in the absence of a ground-plane, a characteristic not available with smaller microstrip or chip antennas. This antenna also offers a large beamwidth, which is favorable since the attitude won't typically be Earth-pointing. Impedance matching can be achieved with microfabricated capacitors and inductors. In an effort to evaluate these practices, we have fabricated candidate capacitors and inductors in the Cornell NanoScale Science \& Technology Facility. For a three layer capacitor with silicon-dioxide as a dielectric, we achieved a capacitance density of $10 \mathrm{nF} / \mathrm{cm}^{2}$. Figure 3.15 shows a photograph of the most recent inductor that we've produced at 20x magnification. This design is based on a three-layer octagonal design by Craninckx and Steyaert [58].

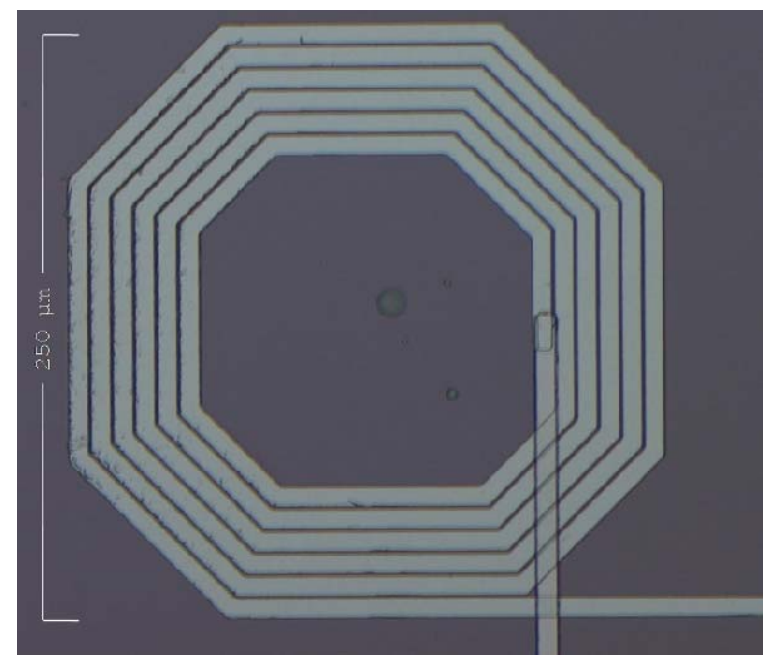

Figure 3.15. A 6 loop, $240 \mu \mathrm{m}$ wide octagonal inductor for the RLC oscillator is shown at $20 \mathrm{x}$ magnification. 


\subsubsection{Power}

Photovoltaic energy harvesting is both passive and based in semiconductor physics, making it a natural choice for power generation in this application. This study baselines silicon-based, first-generation solar cells, which use a single-layer $p-n$ junction diode to pass photovoltaic currents. With strings of individual cells strategically connected in parallel or series, an array can be designed with required voltage and current characteristics to accommodate propulsive, attitude control, or payload requirements. This principle applies equally well to millimeter-scale and macroscopic spacecraft. Commercially available, high-efficiency cells commonly achieve specific power on the order of $200 \mathrm{~W} / \mathrm{kg}$. Integrated solar cells are much less efficient [1]. This inefficiency drives the design to devote most of the available silicon surface to photovoltaics. Likewise, electrochemical batteries are difficult to integrate. It may be that a sprite spacecraft simply ought to be powerless in eclipse. Solar cells produce electric power roughly in proportion to the cosine of the pitch angle. For this reason, sun-pointing attitude solutions offer an important advantage over other attitudes.

The power requirements of the communications downlink exceed the available surface area of the chip. Instead, a simple RC-tank charging circuit can produce periodic bursts of power. Solar power charges a capacitor until a critical voltage is reached, at which point a transistor is activated and the stored energy is released to the communications subsystem. By designing the solar cells in series and parallel combinations, we propose a bus voltage of $50 \mathrm{~V}$. This has the advantage of increasing the energy stored in a capacitor, which goes with voltage squared, and can be accommodated with commonly designed high-voltage transistors. This proposed "bursty" operation has a current-limited charging time of roughly $100 \mathrm{~ms}$ when fully 
illuminated by the sun. It then delivers $10 \mathrm{~mW}$ to the communications subsystem for 12.5 ms. Table 3.2 gives relevant power subsystem parameters.

\begin{tabular}{lcc} 
Table 3.2. Power Budget & & \\
\hline \hline Parameter & Value & Units \\
\hline Solar Cell & & \\
$\quad$ Efficiency & & $\mathrm{mW} / \mathrm{cm}^{2}$ \\
Area & 0.6 & $\mathrm{~cm} 2$ \\
$\quad$ Voltage & 50 & $\mathrm{~V}$ \\
$\quad$ Current & 50 & $\mu \mathrm{A}$ \\
Capacitor & & \\
$\quad$ Charge Density & 500 & $\mathrm{nF} / \mathrm{cm}^{2}$ \\
Area & 0.2 & $\mathrm{~cm} 2$ \\
$\quad$ Capacitance & 100 & $\mathrm{nF}$ \\
Stored Energy & 125 & $\mu \mathrm{J}$ \\
Pulse Length & 12.5 & $\mathrm{~ms}$ \\
Available Power & 10 & $\mathrm{~mW}$ \\
\hline \hline
\end{tabular}

\subsubsection{Payload}

In keeping with this simple, feasible, highly integrated design, the one-way communications beacon serves as a means of transmitting spacecraft position and temperature. The orbit may be estimated by incorporating multiple pulses and an orbit-dynamics model into an orbit-determination filter. Position time histories then allow operators to evaluate the effectiveness of the solar sail. The RF carrier frequency indicates the temperature of the crystal oscillator in the communications circuit. We emphasize that this early phase demo may be followed by other applications, with considerably more sophisticated payloads.

We speculate that simple binary signals could be accommodated easily by modulating the carrier frequency with additional capacitance. For example, with a rudimentary Geiger counter, the transmitter could shift to $434 \mathrm{MHz}$ at times when the 
local impinging radiation exceeded some predefined threshold. Many such signals in a region would depict a time-varying structure within the radiation environment.

\subsubsection{Thermal Control}

In orbit, this spacecraft's low thermal mass results in temperatures between $-130^{\circ} \mathrm{C}$ and $100^{\circ} \mathrm{C}$. Temperature changes across this range can occur in tens of seconds [59]. Thermal stresses associated with eclipses may fatigue the chip where dissimilar metals contact. These risks, along with possible mitigation strategies such as microfabricated radiator fins or silicon carbide circuit technologies [60], have yet to be evaluated. An active means of remediation might be achieved by spinning the bus: tilting the attitude so that the face points toward or away from the sun or the earth at an angle that optimizes combined power and thermal performance.

\subsection{Conclusions}

This paper presents motivation, analytical evaluation, supporting simulation, and sample designs for a millimeter-scale spacecraft with sufficiently low mass to enable solar sailing. The candidate bus is a $1 \mathrm{~cm} \times 1 \mathrm{~cm} \times 25 \mu \mathrm{m}$ silicon IC that conservatively weighs less than $7.5 \mathrm{mg}$. Each conventional spacecraft subsystem is accounted for and described in the context of a Sputnik-inspired temperature-sensing mission.

Applications to formation and sensing are discussed in the context a sun-pointing spacecraft in heliocentric, Earth, and three-body orbits. If paired with two other millimeter-scale spacecraft to form a corner cube, this design can passively point towards the sun with global attitude stability and use SRP to reduce the effect of gravity by $1.0 \%$. This is sufficient to enabling unique formation opportunities. If the 
design uses microfabricated surface facets instead to produce a locally stable sunpointing attitude, this acceleration can be improved to $1.75 \%$ of gravity.

Dramatically reducing a spacecraft's mass by replacing the system with a single integrated circuit enables us to capitalize on these perturbations and feasibly accomplish infinite-impulse missions. We are currently working to complete the demonstration chip and begin evaluating its performance in a terrestrial testbed environment.

\subsection{Acknowledgments}

The authors wish to thank Bernardo Cordovez, Zachary Manchester, and Alfred Ernst of Cornell University for extensive design, fabrication, and testing of communications architectures and Dr. Ben Harris for his support and discussion. J. Atchison wishes to thank Dr. Joseph Burns for his introduction to the celestial mechanics of dust.

This work was funded in part by the National Defense Science Engineering Graduate Fellowship and the NASA Institute for Advanced Concepts (Grant 07605003-071). This work was supported by Sandia National Laboratories through a Center for Integrated Nanotechnologies Grant funded by the Department of Energy. This work was performed in part at the Cornell NanoScale Facility, a member of the National Nanotechnology Infrastructure Network, which is supported by the National Science Foundation (Grant ECS-0335765). 


\section{REFERENCES}

[1] D. Barnhart, T. Vladimirova, M. Sweeting, "Very-Small-Satellite Design for Distributed Space Missions," Journal of Spacecraft and Rockets, Vol. 44, No. 6, 2007, pp. 1294-1306.

[2] D.J. Barnhart, T. Vladimirova, M. Sweeting, "Satellite Miniaturization Techniques for Space Sensor Networks", Journal of Spacecraft and Rockets, Vol. 46, No. 2, 2009, pp. 469-472.

[3] S.W. Janson, "Mass-Producible Silicon Spacecraft for 21st Century Missions," AIAA Space Technology Conference \& Exposition, Albuquerque, NM, 1999.

[4] S.W. Janson, H. Helvajian, K. Breuer, "MEMS, Microengineering and Aerospace Systems", 30th AIAA Fluid Dynamics Conference, AIAA A99-33749, Norfolk, VA, 1999, pp. 1-12.

[5] S.W. Janson, "Micro/Nanotechnology for Micro/Nano/Picosatellites", Space 2003, AIAA 2003-6269, Long Beach, CA, 2003, pp. 1-11.

[6] L.M. Miller, "MEMS for space applications," Proceedings of Society of Photographic Instrumentation Engineers (SPIE), Vol. 3680, Paris, April 1999, pp. 1-13.

[7] C.R. McInnes, "Solar Sailing: Technology, Dynamics and Mission Applications," Springer Verlag, 1999, pp. 1-296.

[8] M. Harwit, "Origins of the Zodiacal Dust Cloud," Journal of Geophysical Research, Vol. 68, No. 8, 1963, pp. 2171-2180.

[9] J. Burns, P. Lamy, S. Soter, "Radiation Forces on Small Particles in the Solar System," Icarus, Vol. 40, 1979, pp. 1-48.

[10] L. Kresak, "Orbital Evolution of the Dust Streams Released from Comets," Astronomical Institutes of Czechoslovakia Bulletin, Vol. 27, No. 1, 1976, pp. 3546.

[11] G. Greschik, "Solar Sail Scalability and a 'Truly Scalable' Architecture: The Space Tow," Journal of Spacecraft and Rockets, Vol. 44, No. 4, 2007, pp. 831839. 
[12] C. Jack, C.S. Welch, "Solar Kites: Small Solar Sails With No Moving Parts", Acta Astronautica, Vol. 40, No. 2-8, 1997, pp. 137-142.

[13] V. Lappas, B. Wie, C.R. McInnes, L. Tarabini, L. Gomes, K. Wallace, "Microsolar Sails for Earth Magnetotail Monitoring", Journal of Spacecraft and Rockets, Vol. 44, No. 4, 2007.

[14] D.A. Vallado, "Solar-Radiation Pressure," Fundamentals of Astrodynamics and Applications, 2nd Ed., Kluwer Academic Publishers, California, 2001, pp 546549.

[15] C. Leinert, E. Grun, "Interplanetary Dust", Physics of the Inner Heliosphere I, Springer-Verlag, Berlin, 1990, pp. 207-256.

[16] P. Staubach, E. Grun, M.J. Matney, "Synthesis of Observations”, Interplanetary Dust, Springer-Verlag, Berlin, 2001, pp. 347-384.

[17] H.P. Robertson, "Dynamical Effects of Radiation in the Solar System", Monthly Notices of the Royal Astronomical Society, Vol. 97, No. 6, 1937, pp. 423-438.

[18] A.A. Shvartsburg, "Multishape Solar Sails: Passive Achievement of the Levitation and Slow Orbiting Stability", AIAA Space Programs and Technologies Conference, AIAA-94-4495, Huntsville AL, September 1994, pp 1-10.

[19] V.V. Radzievskii, "On the Influence of an Anisotropic Re-Emission of Solar Radiation on the Orbital Motion of Asteroids and Meteorites," Astronomicheskii Zhurnal, Vol. 29, 1952, pp. 162-170. [In Russian]

[20] S.J. Paddack, "Rotational Bursting of Small Celestial Bodies: Effects of Radiation Pressure," Journal of Geophysical Research, Vol. 74, 1969, pp. 43794381 .

[21] B. Wie, "Solar Sail Attitude Control and Dynamics: Parts 1 and 2," Journal of Guidance, Control, and Dynamics, Vol. 27, No. 4, 2004, pp. 526-535.

[22] R. Sohn, "Attitude Stabilization by Means of Solar Radiation Pressure," ARS Journal, Vol. 29, No. 5, 1959, pp. 371-373.

[23] M.V. Sykes, D.M. Hunten, F.J. Low, "Preliminary Analysis of Cometary Dust Trails," Advances in Space Research, Vol. 6, No. 7, 1986, pp. 67-78. 
[24] M.V. Sykes, D.J. Lien, “The Tempel 2 Dust Trail,” Icarus, Vol. 86, 1990, pp. 236-247.

[25] M.V. Sykes, R.G. Walker, “Cometary Dust Trails," Icarus, Vol. 95, 1992, pp. 180-210.

[26] H.S. London, "Some Exact Solutions of the Equations of Motion of a Solar Sail With a Constant Setting", American Rocket Society Journal, Vol 30, 1960, pp 198-200.

[27] O. Berg, E. Grun, "Evidence of Hyperbolic Cosmic Dust Particles," Space Research XIII, 1973, pp. 1047-1055.

[28] A. Wehry, I. Mann, "Identification of $\beta$-meteoroids from Measurements of the Dust Detector Onboard the Ulysses Spacecraft," Astronomy and Astrophysics, Vol. 341, No. 1, 1999, pp. 296-303.

[29] C.R. McInnes, "Passive Control of Displaced Solar Sail Orbits", Journal of Guidance, Control, and Dynamics, Vol. 21, No. 6, 1998, pp. 975-982.

[30] S. Gong, J. Li, H. Baoyin, "Passive Stability Design for the Solar Sail on Displaced Orbits", Journal of Spacecraft and Rockets, Vol. 44, No. 5, 2007, pp. 1071-1080.

[31] S. Gong, J. Li, H. Baoyin, "Analysis of Displaced Solar Sail Orbits with Passive Control", Journal of Guidance, Control, and Dynamics, Vol. 31, No. 3, 2008, pp. 782-785.

[32] J.W. Chamberlain, "Depletion of Satellite Atoms in a Collisionless Exosphere by Radiation Pressure," Icarus, Vol. 39, 1979, pp. 286-294.

[33] F. Mignard, "Radiation Pressure and Dust Particle Dynamics," Icarus, Vol. 49, 1982, pp. 347-366.

[34] D.P. Hamilton, A.V. Krivov, "Circumplanetary Dust Dynamics: Effects of Solar Gravity, Radiation Pressure, Planetary Oblateness, and Electromagnetism," Icarus, Vol. 123, Article No. 0175, 1996, pp. 503-523.

[35] R.W. Parkinson, H.M. Jones, I.I. Shapire, "Effects of Solar Radiation Pressure on Earth Satellite Orbits", Science, Vol. 131, No. 3404, 1960, pp. 920-921. 
[36] R. Musen, R. Bryant, A. Bailie, "Perturbations in Perigee Height of Vanguard I", Science, Vol. 131, No. 3404, 1960, pp. 935-936.

[37] I.I. Shapire, H.M. Jones, "Perturbations of the Orbit of the Echo Balloon", Science, Vol. 132, No. 3438, 1960, pp. 1484-1486.

[38]. R. Bryant, "A Comparison of Theory and Observation of the Echo I Satellite", Journal of Geophysical Research, Vol. 66, No. 9, 1961, pp. 3066-3069.

[39] J.C. Van der Ha, V.J. Mode, "Orbital Perturbations and Control by Solar Radiation Forces", Journal of Spacecraft, Vol. 15, No. 2, 1978, pp. 105-112.

[40] J. Burt, M.M. Hedman, M.S. Tiscareno, J.A. Burns, "The Where and Why of Saturn's Inclined 'Charming Ringlet'", American Astronomical Society, Division of Planetary Sciences Meeting, Vol. 40, p. 445.

[41] S.F. Dermott, S. Jayaraman, Y.L. Xu, B.A.S. Gustafson, J.C. Liou, "A Circumsolar Ring of Asteroidal Dust in Resonant Lock with the Earth", Nature, Vol. 269, 1994, pp. 719-723.

[42] D.W. Schuerman, "The Restricted Three-Body Problem Including Radiation Pressure", The Astrophysical Journal, Part 1, Vol. 238, 1980, pp. 337-342.

[43] W.T. Reach, B.A. Franz, J.L. Weiland, et al, "Observational Confirmation of a Circumsolar Dust Ring by the COBE Satellite", Nature, Vol. 374, 1995, pp. 521523.

[44] C.R. McInnes, A.J.C. McDonald, J.F.L. Simmons, E.W. MacDonald, "Solar Sail Parking in Restricted Three-Body Systems" Journal of Guidance, Navigation, and Control, Vol. 17, No. 2, 1994, pp. 399-406.

[45] J.T. Gosling, "Corotating and Transient Solar Wind Flows in Three Dimensions", Annual Review of Astronomy Astrophysics, Vol. 34, 1996, pp. 35-73.

[46] J. Acord, J.C. Nicklas, "Theoretical and Practical Aspects of Solar Pressure Attitude Control for Interplanetary Spacecraft," Progress in Astronautics and Aeronautics, Vol. 13, 1964, pp. 73-91.

[47] E. Polyakhova, "Space Flight Using a Solar Sail - The Problems and the Prospects, Kosmicheskiy Polet Solnechnym Parusom, Moscow, 1986 [In Russian]. 
[48] S. Kirpichnikov, E. Kirpichnikova, E. Polyakhova, "Planar Heliocentric RotoTranslatory Motion of a Spacecraft with a Solar Sail of Complex Shape," Celestial Mechanics and Dynamical Astronomy, Vol. 63, No. 3, 1995, pp. 255269.

[49] C.B. van de Kolk, G.A. Flandro, "Solar Sail Passive Attitude Stability and Control," American Institute of Physics Conference Proceedings, Vol. 552, 2001, pp. 373.

[50] J. Falcovitz, "Attitude Control of a Spinning Sun-Orbiting Spacecraft by Means of a Grated Solar Sail," Center for Space Research, MIT, CSR TR-66-17, Cambridge, MA, 1966.

[51] C.M. Harris and J.W. Wehner., "Solar Torque Control by Using Thin-Film Directionally Sensitive Surfaces," AIAA Guidance, Navigation, and Control Conference, AIAA 2008-7160, Honolulu, Aug 2008

[52] J.W. Wehner, C.M. Harris, M.K. O'Rell, "Solar Torque Control Using Thin Film Directionally Reflective, Emissive, Absorptive, and Transmissive Surfaces”, U.S. Patent No. 6,921,050. July 26, 2005.

[53] K.E. Bean, “Anisotropic Etching of Silicon”, IEEE Transactions on Electron Devices, Vol. 25, No. 10, 1978, pp 1185-1193.

[54] D.H. Lewis, S.W. Janson, R.B. Cohen, "Digital Micropropulsion," Sensors \& Actuators: A.Physical, Vol. 80, No. 2, 2000, pp. 143-154.

[55] J.A. Atchison, M.A. Peck, "A Millimeter-Scale Lorentz Propelled Spacecraft," Proceedings of the AIAA Guidance, Navigation, and Control Conference, AIAA2007-6847, Hilton Head, SC, August 2007.

[56] E.I. Reeves, "Spacecraft Design and Sizing”, Space Mission Analysis and Design, Kluwer Academic Pub, 1999, pp.301-352.

[57] F.J. Dietrich, and R.S. Davies, "Chapter 13: Communications Architecture", Space Mission Analsis and Design, Kluwer Academic Pub, 1999, pp. 533-586.

[58] J. Craninckx, M. Steyaert, "A 1.8-GHz Low-Phase-Noise CMOS VCO Using Optimized Hollow Spiral Inductors," Solid-State Circuits, IEEE Journal of SolidState Circuits, Vol. 32, No. 5, 1997, pp. 736-744. 
[59] D.G. Gilmore, B.E. Hardt, R.C. Prager, E.W. Grob, W. Ousley, "Spacecraft Subsystems - Thermal", Space Mission Analysis and Design, Kluwer Academic Pub, 1999, pp.428-458.

[60] P.G. Neudeck, R.S. Okojie, L.Y. Chen, "High-Temperature Electronics-A Role for Wide Bandgap Semiconductors?," Proceedings of the IEEE, Vol. 90, No. 6, June 2002, pp. 1065-1076. 


\section{CHAPTER 4}

\section{MICROSCALE ATMOSPHERIC RE-ENTRY SENSORS ${ }^{*}$}

\subsection{Abstract}

The area-to-mass dependence of the forces and torques caused by aerodynamic drag implies that aerodynamic accelerations are a function of a body's characteristic length. As characteristic length is reduced, the orbital and angular accelerations increase according to an inverse power law. This suggests that an extremely small body can efficiently aerobrake and aerocapture in the presence of an atmosphere. Likewise, aerothermal and radiative heat transfer scales according to characteristic length, such that sufficiently small bodies maintain lower quasi-equilibrium temperatures throughout an entry maneuver. The combination of these effects enables tens of thousands of metric tons of interplanetary dust to passively enter Earth's atmosphere each year instead of energetically ablating as meteorites.

Seeking to exploit this unique feature of length scaling, the Space Systems Design Studio at Cornell University is developing an extremely small spacecraft dubbed "Sprite." The spacecraft's small size makes this spacecraft extremely sensitive to aerodynamic drag accelerations and therefore able to demonstrate new mission opportunities, such as ablation-free atmospheric entry. The current printed circuit board prototype captures all of the intended functionality in the target application-specific integrated circuit. Recent tests suggest that this prototype can close a communications link over $500 \mathrm{~km}$ with a low power transmitter thanks to matched filter signal processing techniques. Simulations suggest that these low-cost spacecraft can re-enter Earth's atmosphere from a LEO orbit while maintaining a low enough temperature to continuously transmit data.

${ }^{*}$ Reproduced with permission from the Preceedings of the $7^{\text {th }}$ International Planetary Probe Workshop, 12-18 June 2010, Barcelona Spain. 


\subsection{Introduction}

Extremely small bodies in space sometimes experience qualitatively different orbits than larger bodies. It turns out that two-body Keplerian orbital mechanics can fail to describe many recent observations of interplanetary dust particles, whose characteristic sizes are on the order of tens of microns. Dust can be ejected from our solar system by solar radiation pressure $[1,2,3]$, captured or ejected from Jupiter's and Saturn's rings by electromagnetic effects [4,5], and aerocaptured onto Earth's surface without the bright hypersonic ablation characteristic of larger meteors [6,7]. These effects occur because of an inherent length-dependence for many environmental phenomena. This paper asks whether systems designers can exploit such small-body effects to enable ablation-free aerobraking, aerocapture, and re-entry. A swarm of such spacecraft could offer distributed sensing applications, including real-time global studies of the atmosphere.

In pursuit of this and similar questions, Cornell University's Space Systems Design Studio is actively developing an extremely small spacecraft prototype dubbed Sprite. Sprite could roughly be called a "spacecraft-on-a-chip". This architecture reproduces all of the traditional spacecraft hardware on a single semiconducting substrate that is packaged for deployment into the space environment. The expected dimensions for the target application specific integrated circuit package are $1 \mathrm{~cm}$ x $1 \mathrm{~cm} \times 25 \mu \mathrm{m}$.

A number of organizations have focused on developing these technologies $[8,9,10,11,12,13,14,15]$. Barnhart [8] provides an historical summary of these efforts. The motivation for these research efforts follows from the observation that small components and systems on spacecraft offer significant savings in size, mass, and power-three budgets that are particularly expensive on a spacecraft. The trend towards incorporating more and more microfabricated components in spacecraft has already had important impacts in the community: increased accessibility to space, faster development and deployment cycles, the potential for highly distributed sensing missions, and a reduced emphasis on survivability. 
This paper considers an additional benefit of smaller spacecraft size, unique re-entry dynamics. In the context of length-scaling, it addresses the Earth re-entry problem for a small silicon rectangular chip and offer designs and technologies that enable survivable and operational descent. Finally, it describes the Sprite design and offer current fabrication progress.

\subsection{System Modeling}

This section derives the equations of motion for a spacecraft-on-a-chip orbiting in an atmosphere. It considers translational motion, rigid-body motion, as well as thermodynamics.

\subsubsection{Spacecraft-on-a-Chip Geometry}

The spacecraft-on-chip architecture is taken to be a thin, square, flat plate as illustrated in Figure 4.1. A set of right-handed, plate-fixed, orthogonal axes $\left(\boldsymbol{b}_{1}, \boldsymbol{b}_{2}, \boldsymbol{b}_{3}\right)$ are shown in figure, with $\boldsymbol{b}_{3}$ oriented normal to the plate's surface. The base of the plate is a semi-conductor with thin deposited layers of metals and metal-oxides. The thickness $d$ is taken to be much smaller than the side-length $l$

$$
d \ll l \text {. }
$$

The cross-sectional area is then $A_{C}=l^{2}$, and the surface area is $A_{S}=2 l^{2}$. Given a material density $\rho_{m}$, the plate's mass is $m=\rho_{m} l^{2} d$. The inertia is biaxial and can be represented in $\boldsymbol{b}$ axes with

$$
{ }^{b} I=\left[\begin{array}{ccc}
I_{12} & 0 & 0 \\
0 & I_{12} & 0 \\
0 & 0 & I_{3}
\end{array}\right]
$$

where $I_{12}=\frac{1}{12} m l^{2}=\frac{1}{12} \rho_{m} l^{4} d$ and $I_{3}=\frac{1}{6} m l^{2}=\frac{1}{6} \rho_{m} l^{4} d$. 


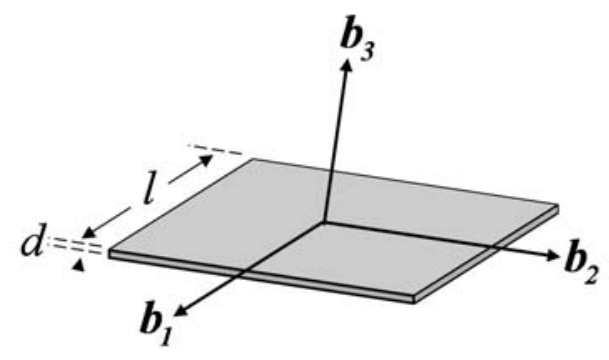

Figure 4.1. Spacecraft-on-a-Chip square flat plate geometry.

\subsubsection{Aerodynamic Drag and Lift Forces}

Aerodynamic drag and lift result from the cumulative molecular interactions between the plate and the atmosphere. Each of these interactions involves an exchange of momentum, and therefore can be modeled as a net force. The standard model for the $\operatorname{drag} \boldsymbol{F}_{\boldsymbol{D}}$ and lift $\boldsymbol{F}_{\boldsymbol{L}}$ components of these forces are given by [16]

$$
\begin{gathered}
\boldsymbol{F}_{\boldsymbol{D}}=-\frac{1}{2} \kappa_{D} A_{C} \rho_{A} v^{2} \hat{\boldsymbol{v}} \\
\boldsymbol{F}_{\boldsymbol{L}}=\frac{1}{2} \kappa_{L} A_{C} \rho_{A} v^{2} \hat{\boldsymbol{L}} .
\end{gathered}
$$

The magnitude of each force is a function of a the cross-sectional area; the local atmospheric density $\rho_{A}$, which encapsulates a strong dependence on altitude; the plate's velocity $v$ squared; and a dimensionless coefficient $\left(\kappa_{D}, \kappa_{L}\right)$. Aerodynamic drag acts opposite the direction of motion $(-\hat{v})$, removing kinetic energy and angular momentum from the plate's motion. By convention, aerodynamic lift is taken to act along $\hat{\boldsymbol{L}}$, a vector perpendicular to $\hat{\boldsymbol{v}}$ and in the plane of $\boldsymbol{F}_{\boldsymbol{D}}$ and $\boldsymbol{b}_{3}$, as illustrated in Figure 4.2. The simpler two dimensional case shown in Figure 4.3 is considered in this paper. 


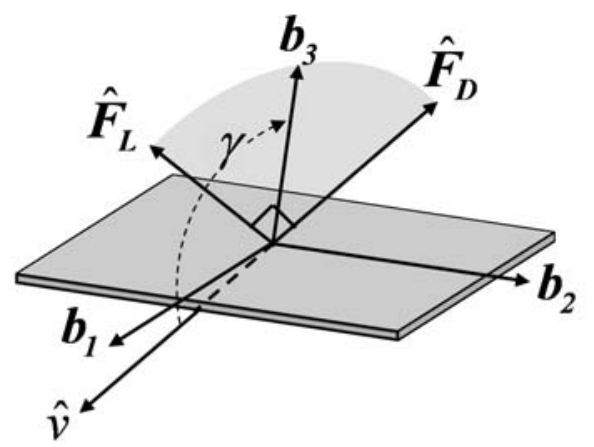

Figure 4.2. Three dimensional orientation of lift and drag forces on a flat plate in a fluid flow

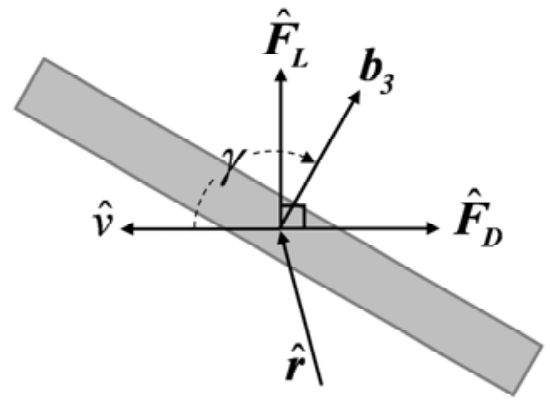

Figure 4.3. Two dimensional orientation of lift and drag forces on a flat plate in a fluid flow

The coefficients of drag $\kappa_{D}$ and lift $\kappa_{L}$ account for the surface interactions between the plate and the atmospheric molecules. To describe these interactions, this research adopts a simple hyperthermal free-molecular flow model given by Storch [17], which ignores spinning and tumbling body effects. In free-molecular flow, the atmosphere is treated as a grouping of individual molecules with statistical properties, rather than as a continuum. As individual molecules impact the plate, they exchange a portion of their momentum (a process called molecular accommodation) before being reflected diffusely or specularly away from the surface.

A flat plate can be thought of as an airfoil because its drag and lift coefficients vary with attitude. Here, the so-called angle-of-attack $\gamma_{A}$ is referenced to $\boldsymbol{b}_{3}, \cos \gamma_{A}=\boldsymbol{b}_{3} \cdot \hat{\boldsymbol{v}}$. In terms of this angle, Storch's equations for $\kappa_{D}$ and $\kappa_{L}$ are [17] 


$$
\begin{gathered}
\kappa_{D}=2\left[\eta_{t}+\eta_{n} \frac{v_{w}}{v} \cos \left(\gamma_{A}\right)+\left(2-\eta_{n}-\eta_{t}\right) \cos ^{2}\left(\gamma_{A}\right)\right] \cos \left(\gamma_{A}\right) \\
\kappa_{L}=\left[\eta_{n} \frac{v_{w}}{v}+\left(2-\eta_{n}-\eta_{t}\right) \cos \left(\gamma_{A}\right)\right] \sin \left(2 \gamma_{A}\right) .
\end{gathered}
$$

The dimensionless coefficients $\eta_{n}$ and $\eta_{t}$ model the molecular accommodation in the plate's normal and tangential directions. These coefficients vary with both the surface properties and the atmospheric properties. The normal component of the thermal velocity of molecules escaping the plate's surface $v_{w}$ is given by [17]

$$
v_{w}=\sqrt{\frac{\pi \Gamma T}{2}}
$$

where $\Gamma$ is the local specific gas constant and $T$ is the surface temperature of the plate. Taking $T$ as the local atmospheric temperature and using circular LEO velocities yields characteristic values for $v_{w} / v$ of roughly 0.05. Figure 4.4 plots Eq. (4.4) and (4.5) over angle-of-attack for these sample conditions. Positive lift is associated with $\cos \left(\hat{\boldsymbol{F}}_{L} \cdot \hat{\boldsymbol{r}}\right)>0$. Both coefficients go to zero at $\hat{\boldsymbol{v}} \perp \boldsymbol{b}_{3}$, the equilibrium attitude. At $\hat{\boldsymbol{v}} \perp \boldsymbol{b}_{\mathbf{3}}$ there is no lift, and drag is maximized, especially since the full square area is leading. Ballistic re-entry is characterized by zero lift throughout the maneuver. As seen in Figure 4.4, lift is zero in the face-on $\left(\gamma_{A}=0\right)$ and edge-on $\left(\gamma_{A}= \pm 90^{\circ}\right)$ plate attitudes.

Storch's model assumes the thickness of the plate is negligible, such that there is negligible lift or drag force in the equilibrium edge-on attitude. Here, the model is augmented to include the small drag force associated with the thin leading edge. This special case uses $\kappa_{D}\left(\gamma_{A}=0\right)$ and $A_{C}=l d$. 


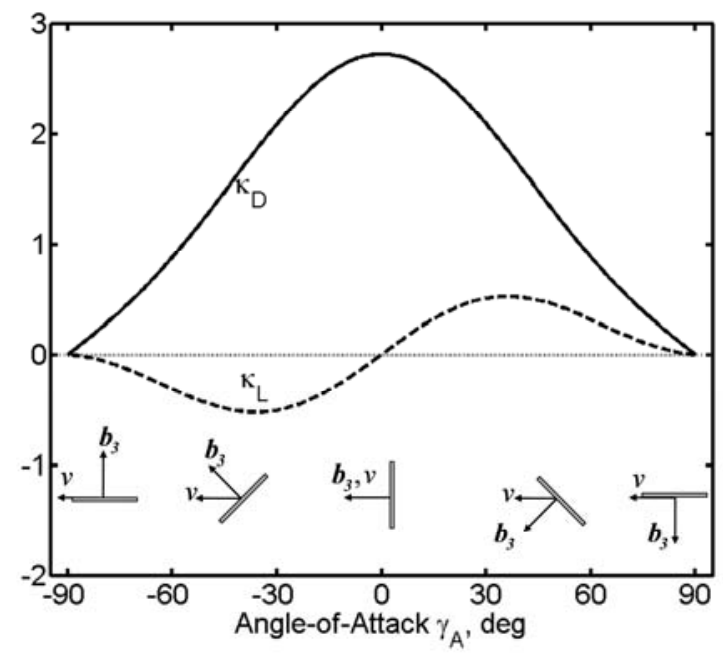

Figure 4.4. Drag and lift coefficients vs. angle-of-attack for a flat plate in hyperthermal free-molecular flow with $\eta_{n}=\eta_{t}=0.7$ and $v_{w} / v=0.05$ [17].

Finally, the orbital equation of motion for the plate throughout atmospheric entry is

$$
\ddot{\boldsymbol{r}}=-\frac{\mu}{r^{2}} \hat{\boldsymbol{r}}+\frac{1}{m}\left(\boldsymbol{F}_{\boldsymbol{D}}+\boldsymbol{F}_{\boldsymbol{L}}\right)
$$

where $\mu$ is the planetary gravitational constant and $\boldsymbol{r}$ is the position vector with magnitude $r$ and direction $\hat{r}$. By Newton's third law, the aerodynamic forces are divided by the plate's mass to produce the relevant accelerations.

\subsubsection{Aerodynamic Drag and Lift Moments}

Given that the plate is much larger than the mean distance between the atmospheric molecules, the net collisions forces $\left(\boldsymbol{F}=\boldsymbol{F}_{\boldsymbol{D}}+\boldsymbol{F}_{\boldsymbol{L}}\right)$ can be modeled as a pressure. This pressure can be said to act at a point, the center of pressure $\boldsymbol{\delta}_{\boldsymbol{C P}}$

$$
\delta_{C P}=\frac{\oint(F \cdot \delta) d \delta}{\oint \delta d \delta}
$$

where $\boldsymbol{\delta}$ is a measurement of position taken with respect to the plate's center-of-mass. For a plate geometry, the net force acts through the center of mass. That is, the force 
should be torque-free. In practice however, any arbitrarily small dislocation of $\boldsymbol{\delta}_{\boldsymbol{C} \boldsymbol{P}}$ causes a torque of the form

$$
\tau=\delta_{C P} \times F
$$

This dislocation could result from imprecision in manufacturing processes, uneven surface corrosion, or uneven flow properties. It could even be intentionally added to produce a known stable equilibrium. In equilibrium $\boldsymbol{\tau}=\mathbf{0}$, a case that is satisfied if $\boldsymbol{F}=$ $\boldsymbol{0}$ or $\boldsymbol{\delta}_{\boldsymbol{C P}} \| \boldsymbol{F}$. The latter case offers the flight realizable situation. This solution is stable if $\left(\hat{\boldsymbol{\delta}}_{C P} \cdot \hat{\boldsymbol{v}}\right)<\mathbf{0}$, which implies that the center-of-mass is "upwind" of the center-ofpressure. Figure 4.5 illustrates this situation with an edge-on attitude. The center-ofpressure is the geometric center of the plate. In this figure, the center-of-mass has been moved left of this point, so that the center-of-mass is "upwind". Small deviations about this equilibrium are stable, as shown in the cases where the plate is pitched clockwise and counterclockwise. In both of these cases, the restoring torque acts opposite the pitch.
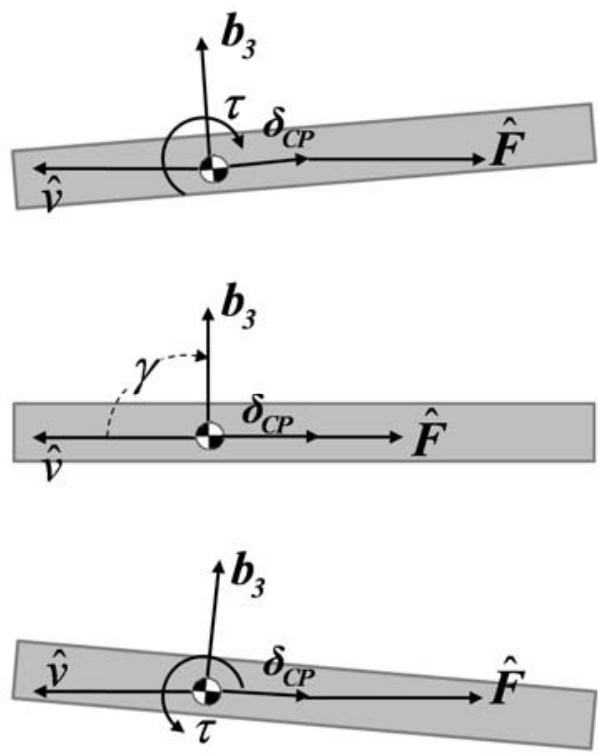

Figure 4.5. Stable equilibrium with center-of-mass "upwind" of center-of-pressure. 
Euler's equation gives the attitude equation of motion in terms of angular velocity $\omega$

$$
\boldsymbol{\tau}=\frac{d}{d t}(\boldsymbol{I} \cdot \boldsymbol{\omega})
$$

where $d / d t$ is the time derivative in an inertial frame. For a plate with constant inertia, the equation of motion becomes

$$
\boldsymbol{I} \cdot \dot{\boldsymbol{\omega}}=\boldsymbol{\omega} \times \boldsymbol{I} \cdot \boldsymbol{\omega}-\boldsymbol{\tau}
$$

Here, the torque is a function of attitude as modeled in Eq. (4.9). Taken in terms of the one dimensional attitude depicted in Figure 4.5, the first order, single variable equation of motion is

$$
I_{12} \ddot{\theta}=\tau
$$

\subsubsection{Thermodynamic Model}

As atmospheric molecules impact the plate, they exchange energy as well as momentum. The plate's kinetic energy is essentially converted to thermal energy through this interaction. A spacecraft maneuver, particularly re-entry, must consider this heat, because the heat rate or the total heat load can destroy components.

This analysis adopts a simple aerothermodynamic model that treats the plate as a single thermal element subjected to convection and radiation. The convection model based off of the seminal convection model by Allen and Eggers [18], but also incorporates aspects of a model by Koppenwallner, Fritsche, and Lips [19] developed for the re-entry of small orbital debris. It also assumes the so-called Reynolds analogy [20], which poses restrictions on its validity at especially high speeds. Despite these restrictions, the simple model adopted here is useful for developing intuition about reentry thermal loading and its dependence on length scales.

Aerothermal convection is assumed to take the form [18]

$$
Q_{c}=\frac{1}{2} C A_{C} \rho_{A} v^{3}
$$


The heat imparted by convection $Q_{c}$ (in units of $\mathrm{J} / \mathrm{s}$ ) is a function of the skin friction coefficient $C$. This coefficient is a function of the local fluid flow properties, which here are accounted for using two aerodynamic similarity parameters: the Knudsen number $K n$ and the Reynolds number $R e$.

The Knudsen number relates the length scale of the plate to the length scale of the local atmospheric molecules. It determines whether the flow can be described best with continuum mechanics or statistical mechanics of the local atmospheric molecules [21]

$$
K n=\frac{\varsigma_{A}}{l} .
$$

where $\zeta_{A}$ is the local mean free path. The Reynolds number relates inertial and viscous forces and assesses turbulence [22]

$$
\operatorname{Re}=\frac{\rho_{A} v l}{\mu_{A}}
$$

where $\mu_{A}$ is the local atmospheric fluid viscosity.

The adopted model then solves for the local skin friction coefficient using the following algorithm:

$$
\begin{aligned}
& K n>10 \quad \text { Free Molecular Flow [19] } \\
& C_{F M}=2 \\
& 10>K n>0.1 \text { Transitional Flow [19] } \\
& C_{T}=\frac{C_{F M}}{1+\left(\frac{C_{C}}{C_{F M}}\right)^{2}} \\
& 0.1>K n \quad \text { Hypersonic Continuum Flow [21] } \\
& R e>2300 \quad \text { Turbulent Flow } \\
& C_{C}=0.0592 R e^{-0.2} \\
& R e<2300 \quad \text { Laminar Flow } \\
& C_{C}=0.664 R e^{-0.5}
\end{aligned}
$$


At each time point, the local flow is characterized, first by Knudsen number and second by Reynolds number. The appropriate model of skin friction coefficient is then evaluated and used to determine the convection heat flow $Q_{c}$.

The plate is also modelled as being capable of radiating heat $Q_{\text {rad }}$ to and from its surroundings. The Stephan-Boltzmann law gives the thermal power emitted from the plate's surface at temperature $T$ to its surroundings at temperature $T_{S}$ [23]

$$
Q_{\text {rad }}=-\sigma \xi A_{S}\left(T-T_{S}\right)^{4}
$$

The emissivity $\xi$ describes the radiation efficiency of the plate's geometry and material.

Given these models for heat transfer, the temperature of the plate with specific heat capacity $c_{p}$ follows the first-order differential equation

$$
\frac{d T}{d t}=\frac{Q_{c}+Q_{r a d}}{m c_{p}} .
$$

This equation can be integrated along the flight path of the plate to give a temperature time-history and assess survivability.

\subsection{Length Scaling}

The square plate geometry design has only two degrees of freedom: length and thickness. In an effort to identify each dimension's impact on performance and survivability, this section considers length-scaling in the above aerodynamic and thermodynamic models.

\subsubsection{Length Scaling in Translational Equations of Motion}

The value of interest, aerodynamic acceleration, depends on the plate's crosssectional area-to-mass ratio $A_{C} / m$

$$
\frac{A_{C}}{m}=\frac{l^{2}}{\rho_{m} d l^{2}}=\frac{1}{\rho_{m} d} .
$$


As the product $\left(\rho_{m} d\right)$ is reduced, the $A_{C} / m$ ratio increases with an inverse-power law. Therefore, for a fixed material density, the plate's thickness is the critical parameter for determining the acceleration associated with aerodynamic forces. The inverse of this ratio appears in the commonly used ballistic coefficient, defined to be a ratio of inertia to aerodynamic drag [19]

$$
\beta \equiv \frac{m}{\kappa_{D} A_{C}}=\frac{\rho_{m} d}{\kappa_{D}}
$$

This ratio determines a body's drag-limited lifetime in LEO. Low values of $\beta$ correspond to satellites whose orbits are highly affected by atmospheric drag, and consequently de-orbit more quickly than bodies with high $\beta$. Typical spacecraft have ballistic coefficients on the order of 10 to $100 \mathrm{~kg} / \mathrm{m}^{2}$ [24]. The ballistic coefficient also drives a body's terminal velocity

$$
v_{T}=\sqrt{\frac{2 g}{\rho_{A} \beta}} .
$$

\subsubsection{Length Scaling in Rotational Equations of Motion}

The attitude or rigid-body equation of motion gives aerodynamic angular accelerations as $\boldsymbol{I}^{-1} \boldsymbol{\tau}$. The torque, given in Eq. (4.9) is roughly a function of $A_{C} l$. The magnitude of the angular accelerations are then proportional to $\left(\rho_{m} l d\right)^{-1}$. As the length and thickness of the plate are reduced, the angular accelerations associated with aerodynamic torques increase in magnitude.

\subsubsection{Length Scaling in Thermodynamics}

The heat models $Q_{c}$ and $Q_{\text {rad }}$ are both a function of area explicitly. In terms of the quantity of interest, the temperature, the heat transfer rates are divided by mass. So in the case of radiation, $Q_{\text {rad }}$ depends on the plate's surface-area-to-mass $A_{S} / m$ 


$$
\frac{A_{S}}{m}=\frac{2 l^{2}}{\rho_{m} d l^{2}}=\frac{2}{\rho_{m} d}
$$

In the case of forced convection, the length-dependence is a function of the flow regime as well. The flow regime is determined by the Knudsen number, which is proportional to $l^{-1}$. In free molecular flow, $Q_{c}$ depends on $A_{C} m$ and therefore $d^{-1}$. In continuum flow, the skin friction coefficient depends on powers of $R e$. Laminar flow results in $Q_{c} \propto l^{1.5}$ and

$$
\frac{d T}{d t} \propto \frac{1}{\rho_{m} d l^{0.5}} .
$$

Turbulent flow results in $Q_{c} \propto l^{1.8}$ and

$$
\frac{d T}{d t} \propto \frac{1}{\rho_{m} d l^{0.2}}
$$

Recognizing that $t \ll l$, both thickness and side-length are relevant design parameters when considering thermal survivability in continuum flow. When considering radiation or free molecular flow, thickness is the only critical parameter.

\subsubsection{Survivability of Dust Grains}

If a body is small enough, it may be capable of decelerating and radiating heat efficiently enough to maintain a survivable temperature. Indeed, this phenomenon is observed in nature $[6,7]$. Each year, thousands of metric tons of small interplanetary dust particles reach the Earth's surface unaffected while larger meteoroids ablate as meteorites [25].

The key to a dust grain's low-temperature entry is the altitude range in which its entry velocity is primarily reduced. Thanks to a high sensitivity to aerodynamic drag, some dust grains decelerate in the upper atmosphere where free-molecular flow heating occurs. Here, the atmospheric density is low enough that the product of $\rho v^{3}$, which occurs in the aerothermal heating model, doesn't reach ablative values [26]. By the 
time that the dust grain reaches the thicker continuum atmosphere, its velocity is at or near terminal velocity, a value that typically low for dust.

The chip architecture is similar to a dust grain, in that it achieves high $\mathrm{A} / \mathrm{m}$. However, one key difference is that dust grains have a small characteristic length in the fluid flow properties, specifically the Knudsen number. For a flat plate in the flow, the side-length is the applicable characteristic length. This means that the plate enters continuum flow much sooner than a dust grain, for the same altitude and velocity profile.

\subsection{Results}

The three equations of motion (Eqs (4.7), (4.12) and (4.17)) have been simulated for a variety of cases. Each simulation uses the US 1976 Standard Atmosphere Model [27] for local atmospheric properties. Other parameters are listed in Table 4.1. The material of the plate is taken to be silicon. The initial orbit is taken to be a circular orbit in the plane of Earth's equator, with an altitude of $350 \mathrm{~km}$. The end condition is taken to be an altitude of $10 \mathrm{~km}$ above Earth's surface. Many electronic components can survive and operate at temperatures up to $100^{\circ} \mathrm{C}$, so this is taken to be the survivability limit.

\begin{tabular}{|c|c|c|}
\hline Parameter & Value & \\
\hline$\rho$ & $2330 \mathrm{~kg} / \mathrm{m}^{3}$ & [28] \\
\hline$\zeta$ & 0.85 & \\
\hline$c_{p}$ & $678 \mathrm{~J} /(\mathrm{kg} \mathrm{K})$ & [28] \\
\hline$h_{0}$ & $350 \mathrm{~km}$ & \\
\hline$v_{0}$ & $7697.0 \mathrm{~km} / \mathrm{s}$ & \\
\hline$T_{S}$ & $255 \mathrm{~K}$ & [23] \\
\hline$\mu$ & $398658.366 \mathrm{~km}^{3} / \mathrm{s}^{2}$ & \\
\hline
\end{tabular}




\subsubsection{Parametric Search}

Two numerical parametric searches were employed in an effort to assess the design space of survivable plate dimensions for ballistic re-entry. The simulations each assume that the plate maintains a fixed attitude, $\gamma_{A}=90^{\circ}$ or $0^{\circ}$. These attitudes represent edgeon and face-on ballistic re-entries respectively, and represent bounding conditions. That is, Section 4.3.3 argues that the edge-on heading is stable, and therefore the face-on heading represents an unlikely but possible worst-case heading. The simulations each test a different choice of side length, thickness, and orientation. Side length $l$ is varied between $0.5 \mathrm{~cm}$ and $10 \mathrm{~cm}$ and the thickness $d$ is varied between $25 \mu \mathrm{m}$ and $1 \mathrm{~mm}$, for a total of 286 simulations.

Figure 4.6 and Figure 4.7 give the results of the maximum temperature that each simulation reaches for the face-on and edge-on attitudes. The maximum temperature is dependent strongly on both side length and thickness. The simulations using a face-on heading do not yield survivable maximum temperatures. Simulations for the edge-on heading show much lower temperatures, with large dimensioned plates maintaining an equilibrium temperature of $T_{s}$ due to thermal radiation from the Earth.

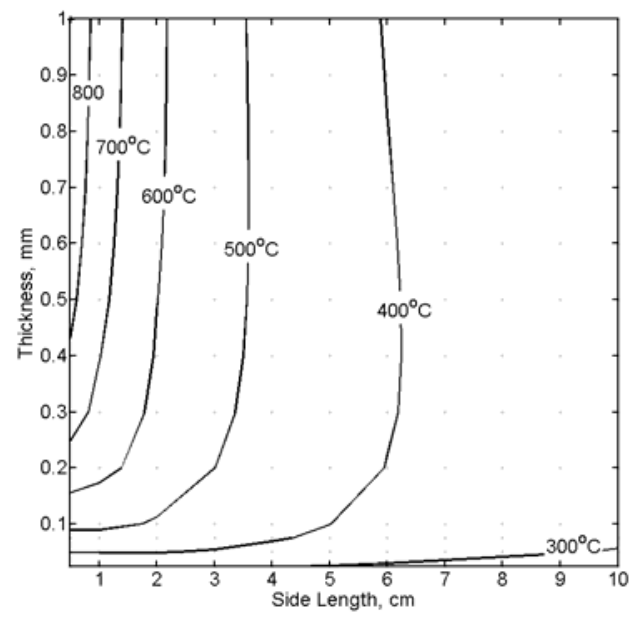

Figure 4.6. Maximum temperature during re-entry maneuver for the face-on attitude.

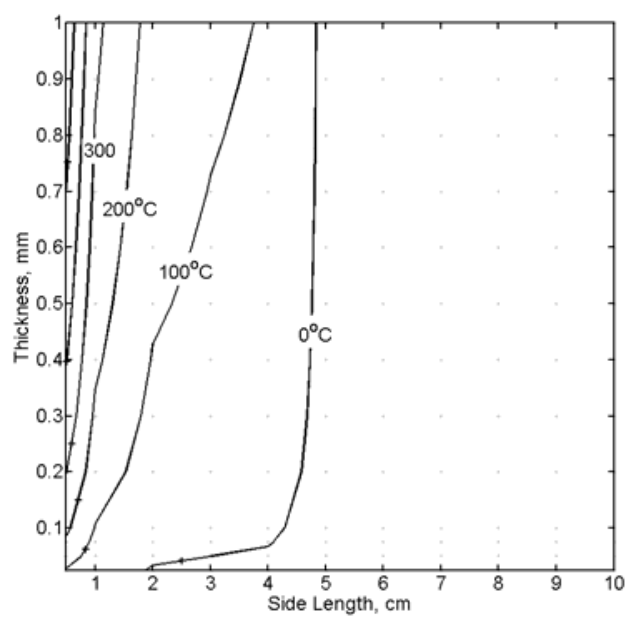

Figure 4.7. Maximum temperature during re-entry maneuver for the edge-on attitude. 


\subsubsection{Sprite Re-Entry Simulations}

The Monte Carlo simulations suggest that the target Sprite architecture of $l=1 \mathrm{~cm}$ and $d=25 \mu \mathrm{m}$ can survive an edge-on re-entry, though larger dimensions are possible and even beneficial. Focusing on this choice of dimensions, the face-on and edge-on cases are evaluated more carefully in an effort to describe the key differences between the two headings.

Figure 4.8 through Figure 4.11 give relevant results for the face-on heading. This heading yields $A_{C} / m=17.2, A_{S} / m=34.3$, and $\beta=0.02$. The total re-entry process takes only $5 \mathrm{hr}$. Immediately after beginning the simulation, the plate's altitude rapidly drops, reaching a peak drag acceleration of about $100 \mathrm{~m} / \mathrm{s}^{2}$. The peak temperature of $300^{\circ} \mathrm{C}$ occurs with the peak drag acceleration at an altitude of approximately $150 \mathrm{~km}$. At other times, the thermal radiation of the Earth keeps the steady state temperature at the assumed value of $T_{s}=-18^{\circ} \mathrm{C}$. The Knudsen number time-history shows that peak heating occurs during the free molecular flow regime and transitional flow regime. The Reynolds number time-history suggests that the continuum flow stays laminar.

Figure 4.12 to Figure 4.17 give time histories for the edge-on re-entry maneuver. This heading yields $A_{C} / m=0.043, A_{S} / m=34.3$ and $\beta=9.0$. The lower value of $A_{C} / m$ causes the re-entry to take 322 hours, during which time it reaches a peak temperature of only $28^{\circ} \mathrm{C}$. The magnitude of drag acceleration is reaches almost $100 \mathrm{~m} / \mathrm{s}^{2}$, but occurs during turbulent continuum flow conditions, when the heat transfer is lower than the free molecular flow case. The key difference between the two orientations appears to be the flow condition at which maximum deceleration occurs.

This essentially depicts the difference between the entry of dust and the entry of typical spacecraft systems. The face-on attitude re-enters in the free-molecular flow regime, as dust does. As a result, it only heats to a few hundred degrees Celsius, rather than the thousands of degrees typical for re-entry systems. The edge-on attitude enters more slowly, during which time it experiences maximum heating in the continuum 
regime. The edge-on attitude experiences low temperatures because it is decelerates at lower rates.

The assumption that the Sprite can be treated as single thermal element may give unreasonable results for the edge-on architecture. The leading edge will likely be subjected to the vast majority of the aerothermal heat input, and this could lead to localized temperatures exceeding the results presented here.

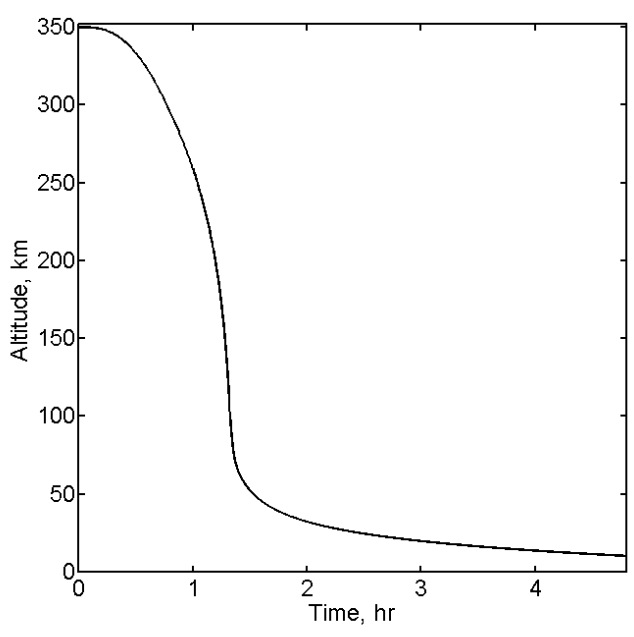

Figure 4.8. Altitude time-history for a face-on re-entry of the target Sprite architecture.

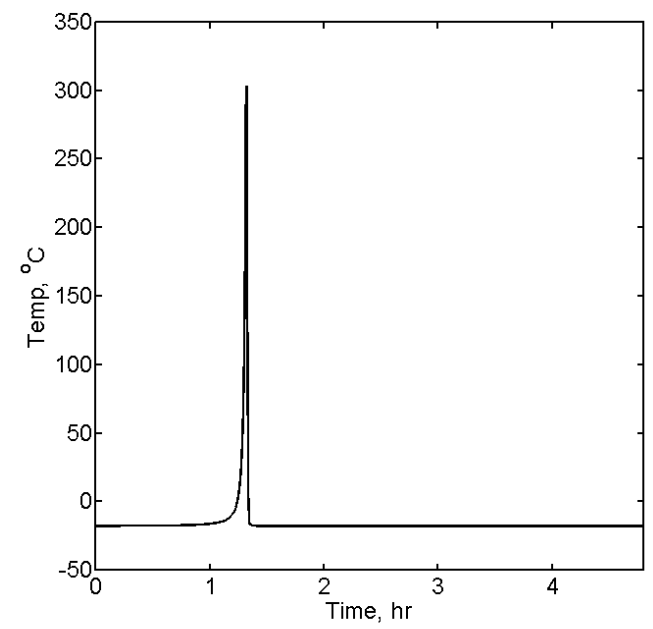

Figure 4.10. Temperature time-history for a face-on re-entry of the target Sprite architecture.

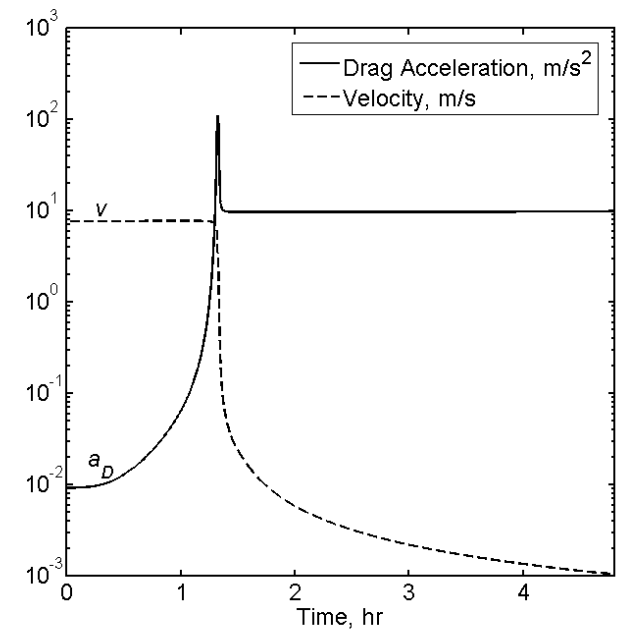

Figure 4.9. Drag acceleration and velocity time-histories for a face-on re-entry of the target Sprite architecture.

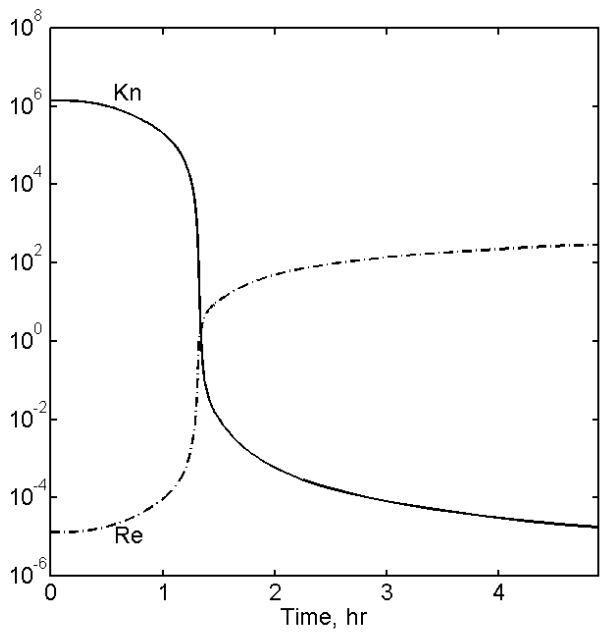

Figure 4.11. Knudsen number and Reynolds number time-histories for a faceon re-entry of the target Sprite architecture. 


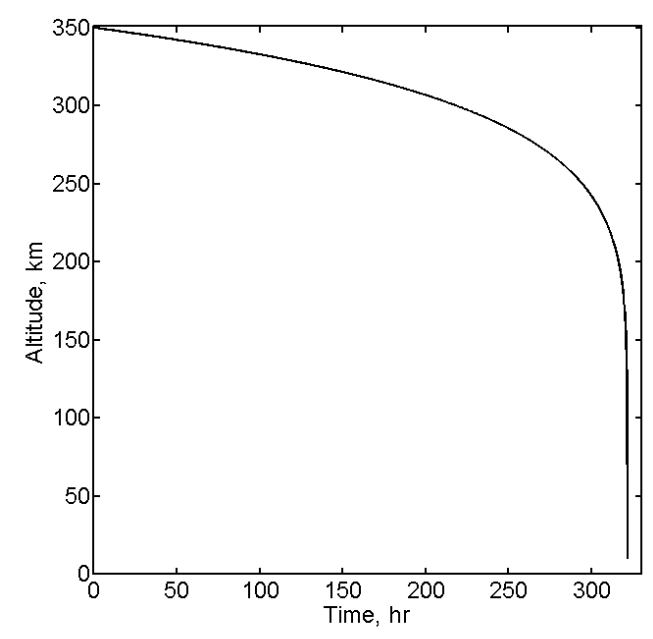

Figure 4.12. Altitude time-history for an edge-on re-entry of the target Sprite architecture.

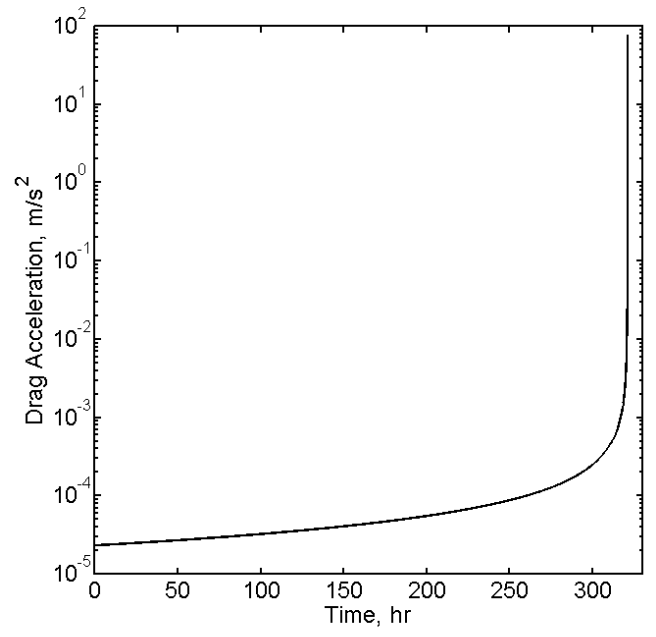

Figure 4.14. Drag acceleration timehistory for an edge-on re-entry of the target Sprite architecture.

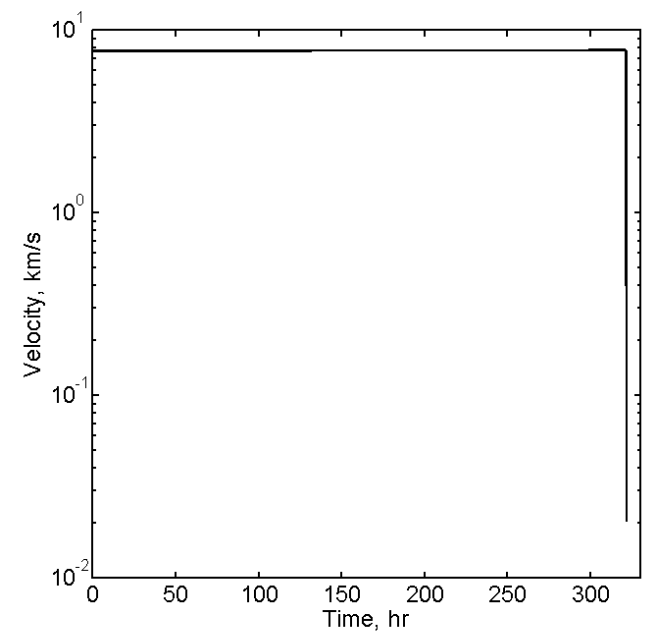

Figure 4.13. Velocity time-history for an edge-on re-entry of the target Sprite architecture.

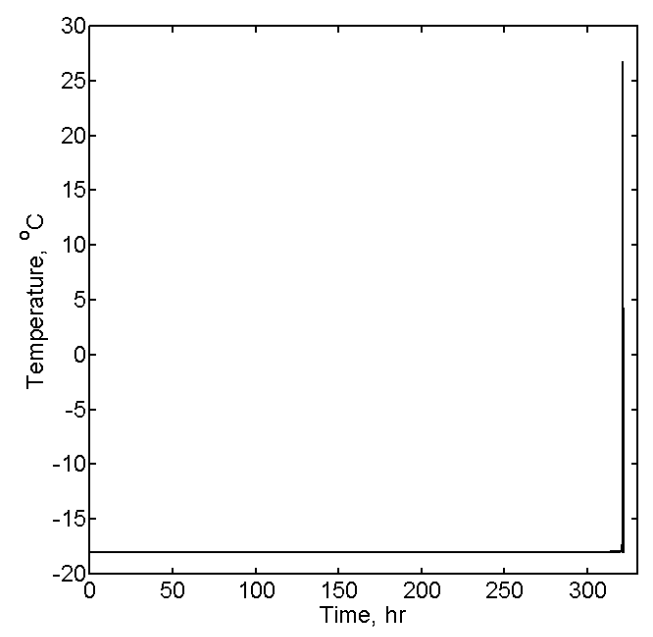

Figure 4.15. Temperature time-history for an edge-on re-entry of the target Sprite architecture. 


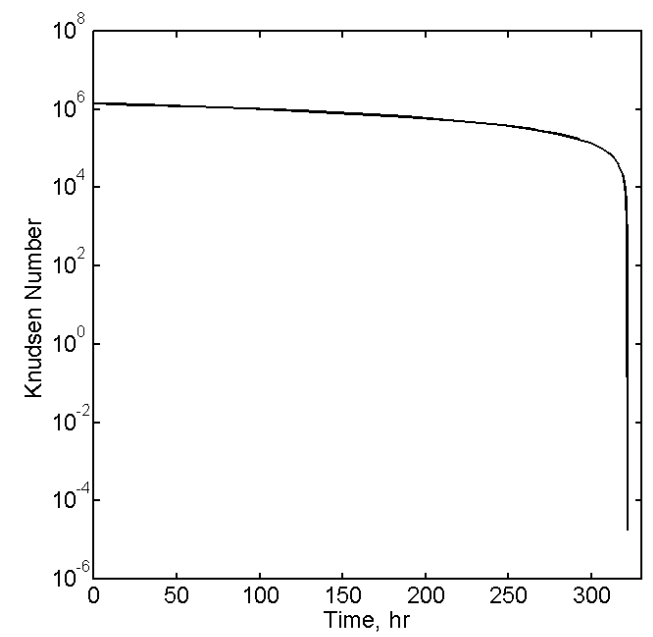

Figure 4.16. Knudsen number time history for an edge-on re-entry of the target Sprite architecture.

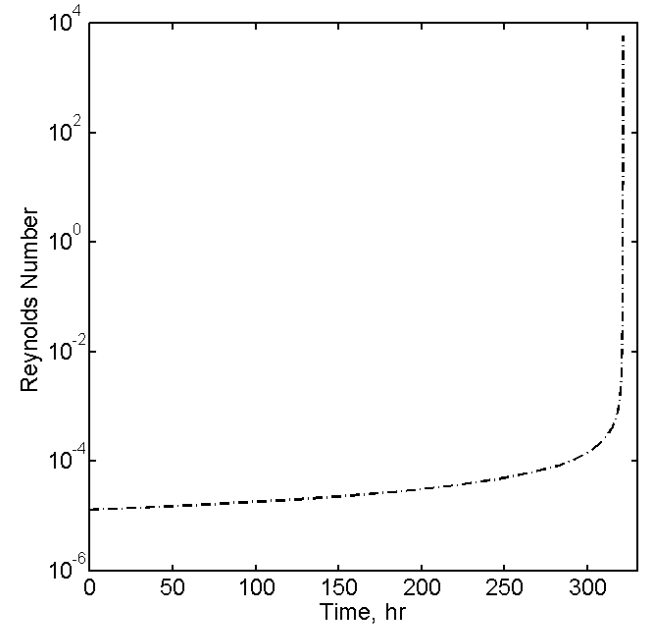

Figure 4.17. Reynolds number time history for an edge-on re-entry of the target Sprite architecture.

\subsection{Sprite Spacecraft Design}

The earliest Sprite prototypes have focused on three primary subsystems: structure, power, and communications. The current printed-circuit-board (PCB) version reproduces the desired functionality using commercial off-the-shelf components. Each aspect of this first design, shown in Figure 4.18, is traceable to a smaller target size of 1 $\mathrm{cm} \times 1 \mathrm{~cm} \times 25 \mu \mathrm{m}$.

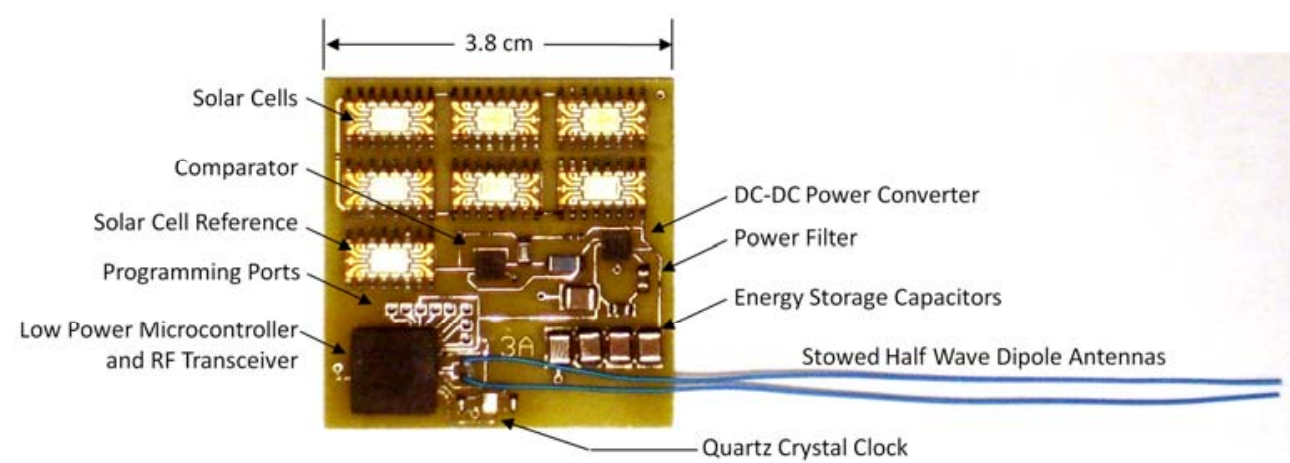

Figure 4.18. Current large-scale PCB prototype 


\subsubsection{Structure}

The structure must mechanically support the functional components within the target form factor. The PCB prototype measures $3.8 \mathrm{~cm} \mathrm{x} 3.8 \mathrm{~cm}$ and has been manufactured using standard PCB techniques onto a $1.5 \mathrm{~mm}$ thick FR-4 substrate. The back side of the structure serves as a ground plane for the electronics and antenna. The total mass is 8 grams, resulting in a maximum cross sectional $A_{C} / m$ of $0.18 \mathrm{~m}^{2} / \mathrm{kg}$. The objective Sprite is a thin ASIC architecture. Table 4.2 gives the relevant parameters for the current PCB and objective ASIC Sprite structures.

\begin{tabular}{cccc}
\multicolumn{4}{l}{ Table 4.2. Sprite Structural Parameters } \\
\hline & $\begin{array}{c}\text { Current } \\
\text { PCB }\end{array}$ & Objective & Units \\
\hline$l$ & 3.8 & 1 & $\mathrm{~cm}$ \\
$m$ & 8 & 0.0075 & $\mathrm{gm}$ \\
$d$ & 1.5 & 0.025 & $\mathrm{~mm}$ \\
$I_{12}$ & $1.925 \times 10^{-5}$ & $1.25 \times 10^{-10}$ & $\mathrm{~kg} \cdot \mathrm{m}^{2}$ \\
$I_{3}$ & $9.627 \times 10^{-6}$ & $6.25 \times 10^{-11}$ & $\mathrm{~kg} \cdot \mathrm{m}^{2}$ \\
\hline \hline
\end{tabular}

\subsubsection{Power}

Power is sourced using a total of seven solar cells for operation. The power requirements of the communications hardware would require too many cells to maintain the required form factor; so, the design uses "bursty" operation. Six of the solar cells charge a $20 \mu \mathrm{F}$ storage capacitor. When this capacitor is charged to roughly $30 \mathrm{~V}$, the energy discharges through a filtered DC-DC power converter. This operation is controlled by a comparator sourced by the seventh solar cell. The output of this power converter is a $3.3 \mathrm{~V}$ square wave made available to the communications subsystem. This power is sufficient to boot up the communications hardware and transmit a beacon signal. 
Figure 4.19 and Figure 4.20 give voltage time-histories for the power subsystem's operation under a sun simulator. Figure 4.19 shows the voltage across the energystorage capacitor and the filtered voltage from the DC-DC power converter. "In" denotes the voltage time history across the energy-storage capacitor. "Out" denotes the voltage from the filtered DC-DC converter. The capacitor requires $1 \mathrm{~s}$ to charge up to $29 \mathrm{~V}$, after which it is discharged down to $17 \mathrm{~V}$. This high voltage operation offer increased energy storage for a given capacitor, since capacitance goes with voltagesquared. Figure 4.20 is a close-up of the filtered voltage output. The pulse lasts a total of $18 \mathrm{~ms}, 2 \mathrm{~ms}$ of which are used to boot up the transmitter. The remainder of the power is used for continuous operation of the communications subsystem. After the pulse stops, the remaining power dissipates slowly until the next pulse. Given that the transmitter is powered for $18 \mathrm{~ms}$ every $1 \mathrm{~s}$, power subsystem offers a duty cycle of $1.8 \%$. Table 4.3 gives the relevant power subsystem parameters.

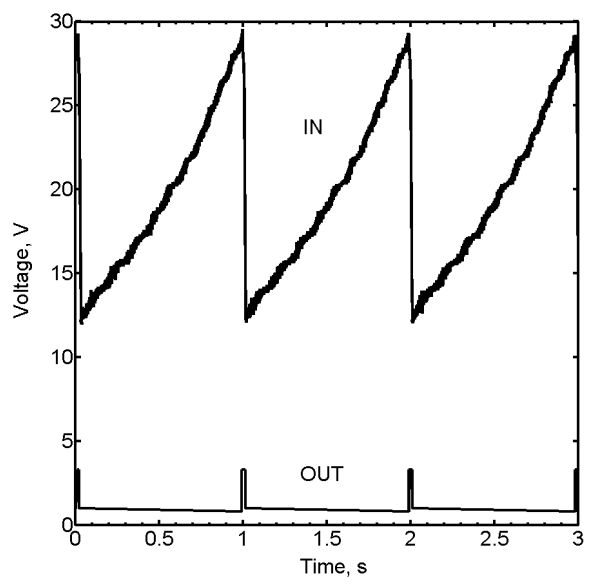

Figure 4.19. Power subsystem performance in a sun-simulator.

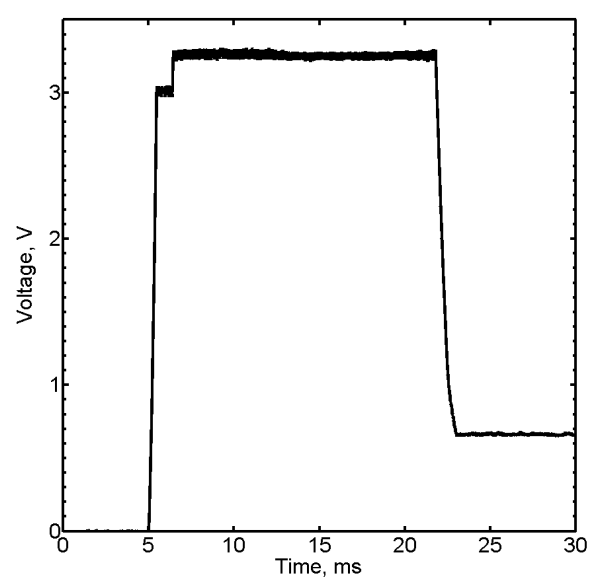

Figure 4.20. Filtered DC power subsystem output. 
Table 4.3. Sprite Power Subsystem Parameters

\begin{tabular}{lcc}
\hline Parameter & Value & Units \\
\hline Solar Cells & 6 & \\
Voltage & 32 & $\mathrm{~V}$ \\
Current & 50 & $\mu \mathrm{A}$ \\
Capacitor & 20 & $\mu \mathrm{F}$ \\
$\quad$ Min/Max Voltage & $18 / 30$ & $\mathrm{~V}$ \\
Charge Time & 1.0 & $\mathrm{~s}$ \\
Released Energy & 6.4 & $\mathrm{~mJ}$ \\
Pulse Length & 18 & $\mathrm{~ms}$ \\
DC-DC Efficiency & 80 & $\%$ \\
Filtered DC Output & & \\
$\quad$ Supply Voltage & 3.3 & $\mathrm{~V}$ \\
$\quad$ Supply Current & 86 & $\mathrm{~mA}$ \\
Power Consumption & & \\
Microcontroller Power & -0.6 & $\mathrm{~mA}$ \\
$\quad$ Transmitter Power & -29 & $\mathrm{~mA}$ \\
Margin & 56 & $\mathrm{~mA}$ \\
\hline \hline
\end{tabular}

\subsubsection{Communications}

When activated, a low-power microcontroller powers up a transmitter IC and sends instructions to transmit a predetermined $16 \mathrm{~ms}$ signal at $900 \mathrm{MHz}$. The signal is clocked using an external quartz oscillator and impedance matched to a centerfed halfwave dipole. The chief advantage of the dipole antenna selection is performance in spite of having a negligible ground plane.

Using minimum shift keyed pseudo-random noise (PRN) encoding, the system achieves an effective matched filter gain of roughly $22 \mathrm{~dB}$. The transmitted signal (a 160 bit PRN sequence) is known by both the transmitter and receiver in advance. This scheme enables the receiving station to search for the specific binary sequence below the noise floor, as in the case of a standard global positioning system signal. A second advantage is the ability for multiple chips to transmit at the same frequency. The cost of this scheme is a very slow datarate. 
Table 4.3 gives the communications subsystem parameters. The subsystem transmits a linearly polarized signal with $10 \mathrm{~mW}$ of effective isotropic radiated power. For a 500 $\mathrm{km}$ altitude transmitting to a ground station with $18 \mathrm{~dB}$ of antenna gain, the communications link closes with a $5 \mathrm{~dB}$ margin. The carrier-to-noise ratio is 0.07 , suggesting that the encoded signal is well below the noise floor. The encoded signal has a ratio of received energy-per-bit to noise-density $\left(\mathrm{E}_{\mathrm{b}} / \mathrm{N}_{0}\right)$ of 10 , implying that the 160 bit sequence can be inferred by the ground station, and thus it can achieve the 22 $\mathrm{dB}$ matched filtering scheme.

Table 4.4. Sprite Communications Subsystem Parameters

\begin{tabular}{lcc}
\hline Parameter & Value & Units \\
\hline Transmitter & -20 & $\mathrm{dBW}$ \\
$\quad$ Power & 0 & $\mathrm{~dB}$ \\
$\quad$ Antenna Gain & 900 & $\mathrm{MHz}$ \\
$\quad$ Frequency & 1 & $\mathrm{MHz}$ \\
$\quad$ Bandwidth & 16 & $\mathrm{~ms}$ \\
$\quad$ Pulse Length & 10 & $\mathrm{kHz}$ \\
$\quad$ Chip Rate & & \\
Orbit & 500 & $\mathrm{~km}$ \\
$\quad$ Altitude & $+/-20$ & $\mathrm{deg}$ \\
$\quad$ Overhead Arc-Length & -146 & $\mathrm{~dB}$ \\
$\quad$ Free Space Loss & -2 & $\mathrm{~dB}$ \\
$\quad$ Atmospheric Attenuation & & \\
Receiver & 18 & $\mathrm{~dB}$ \\
$\quad$ Receiver Gain & 300 & $\mathrm{~K}$ \\
$\quad$ Noise Temperature & -142 & $\mathrm{dBW}$ \\
$\quad$ Noise Power & 3 & $\mathrm{~dB}$ \\
$\quad$ Polarization Loss & 2 & $\mathrm{~dB}$ \\
Margin & -154 & $\mathrm{dBW}$ \\
Received Power & 0.07 & \\
Carrier to Noise Ratio & 22 & $\mathrm{~dB}$ \\
Matched Filter Gain & 10 & \\
E $/ \mathrm{N}_{0}$ & 11 & \\
Signal to Noise Ratio & & \\
\hline \hline
\end{tabular}




\subsection{Conclusions}

This research considers length-scaling in the atmospheric re-entry problem. It focuses on a geometry relevant to spacecraft-on-chip research, a thin square plate. Simulations of the equations of motion and the aerothermal heating environment suggest that certain choices of side-length, thickness, and plate orientation may survive the re-entry without reaching high temperatures and ablation. By considering both the stable edge-on plate heading and unstable face-on heading, the simulations can roughly bound the plate's dynamic and thermal behavior. Results suggest that the plate may stay cool enough such that on-board electronics could continuously operate through the maneuver. This possibility offers new missions concepts with applications for high altitude atmospheric study.

In pursuit of these types of opportunities, Cornell University is developing an extremely small solid-state spacecraft architecture known as Sprite. The program is currently prototyping the functionality in a printed circuit board package and collaborating with experts in microfabrication to move forward with an application specific integrated circuit package. The driving design goal is to achieve data transmission over LEO distances using Sprite's low power transmitter. The solution demonstrated here incorporates matched filter signal processing techniques to close the link.

This research suggests that small chip-scale spacecraft may experience low temperature re-entry by virtue of their size only. Aside from continuing to develop the Sprite bus, future efforts will be focused on evaluating passive lifting re-entries, refining the aerothermal heating model, and considering other planetary atmospheres. 


\section{REFERENCES}

[1] Harwit, M., "Origins of the Zodiacal Dust Cloud," Journal of Geophysical Research, Vol. 68, No. 8, 1963, pp. 2171-2180.

[2] Burns, J., Lamy, P., and Soter, S., "Radiation Forces on Small Particles in the Solar System," Icarus, Vol. 40, 1979, pp. 1-48.

[3] Kresak, L., "Orbital Evolution of the Dust Streams Released from Comets," Astronomical Institutes of Czechoslovakia Bulletin, Vol. 27, No. 1, 1976, pp. 3546.

[4] Colwell, J.E., Horanyi, M., and Grun, E., "Capture of Interplanetary and Interstellar Dust by the Jovian Magnetosphere," Science, Vol. 280, April 1998, pp. 88-91.

[5] Hamilton, D.P. and Burns, J.A., "Ejection of Dust from Jupiter's Gossamer Ring," Nature, Vol. 364, August 1993, pp. 695-699.

[6] Whipple, F.L., "Theory of micro-meteorites. I. In an isothermal atmosphere," Proceedings of the National Academy of Sciences USA, Vol. 86, 1950, pp. 687695.

[7] Beech, M., "Finite-size Corrections to the Atmospheric Heating of Micrometeorites," Monthly Notices of the Royal Astronomical Society, Vol. 402, No. 2, 2010, pp. 1208-1212.

[8] Barnhart, D., Vladimirova, T., Sweeting, M., "Very-Small-Satellite Design for Distributed Space Missions," Journal of Spacecraft and Rockets, Vol. 44, No. 6, 2007, pp. 1294-1306.

[9] Barnhart, D., Vladimirova, T., Sweeting, M., "Satellite Miniaturization Techniques for Space Sensor Networks," Journal of Spacecraft and Rockets, Vol. 46, No. 2, 2009, pp. 469-472.

[10] Atchison, J.A., and Peck, M.A., "A Passive, Sun-Pointing, Millimeter-Scale Solar Sail,” Acta Astronautica, Vol. 67, No. 1-2, 2010, pp. 108-121.

[11] Atchison, J.A., and M.A. Peck, "A Millimeter-Scale Lorentz-Propelled Spacecraft," AIAA Guidance, Navigation, and Control Conference and Exhibit, AIAA-2007-6847, South Carolina, August 20-23, 2007.

[12] Janson, S.W., "Mass-Producible Silicon Spacecraft for 21st Century Missions," AIAA Space Technology Conference \& Exposition, Albuquerque, NM, 1999. 
[13] Janson, S.W., Helvajian, H., Breuer, K., "MEMS, Microengineering and Aerospace Systems," 30th AIAA Fluid Dynamics Conference, AIAA A99-33749, Norfolk, VA, 1999, pp. 1-12.

[14] Janson, S.W., "Micro/Nanotechnology for Micro/Nano/Picosatellites," Space 2003, AIAA 2003-6269, Long Beach, CA, 2003, pp. 1-11.

[15] Miller, L.M., "MEMS for space applications," Proceedings of Society of Photographic Instrumentation Engineers (SPIE), Vol. 3680, Paris, April 1999, pp. $1-13$.

[16] Vallado, D. A., "Chapter 8.6: Disturbing Forces," Fundamentals of Astrodynamics and Applications, 2nd Ed., Microcosm Press, El Segundo, CA, 2004, pp. 550-551.

[17] Storch, J.A., "Aerodynamic Disturbances on Spacecraft in Free-Molecular Flow," The Aerospace Corporation, Report No TR-2003(3397)-1, October 2002, pp. 170 .

[18] Allen, H.J., and Eggers, A.J., "A Study of the Motion and Aerodynamic Heating of Missiles Entering the Earth's Atmosphere at High Supersonic Speeds," NASA Technical Report \#1381, 1958.

[19] Koppenwallner, G., Fritsche, B., and T. Lips, "Survivability and Ground Risk Potential of Screws and Bolts of Disintegrating Spacecraft during Uncontrolled reentry," Proceedings of the Third European Conference on Space Debris, 19 - 21 March 2001, Darmstadt, Germany. Ed.: Huguette Sawaya-Lacoste. ESA SP-473, Vol. 2, Noordwijk, Netherlands: ESA Publications Division, pp. 533 - 539.

[20] Regan, F.J., and S.M. Anandakrishnan, "Chapter 7.5 Heat Transfer and Dynamics," Dynamics of Atmospheric re-entry, $1^{\text {st }} \mathrm{Ed}$, American Institute of Aeronautics and Astronautics, 1993, pp. 216-222.

[21] Hirshcel, E.H., Basics of Aerothermodynamics, $1^{\text {st }}$ Ed, Springer-Verlag, Berlin, 2005.

[22] Griffin, M.D., and J.R. French, “Atmospheric Entry," Space Vehicle Design, $2^{\text {nd }}$ Ed., AIAA Education Series, 2004, pp. 273-320.

[23] Gilmore, D.G., Hardt, B.E., Prager, R.C., Grob, E.W., and Ousley, W., "Spacecraft Subsystems - Thermal," Space Mission Analysis and Design, Kluwer Academic Pub, 1999, pp. 428-458.

[24] Griffin, M.D., and J.R. French, "Chapter 11 Atmospheric Entry," Space Vehicle Design, 2nd Ed., AIAA, Reston VA, pp. 305-320.

[25] Love, S.G., and D.E. Brownlee, "A Direct Measurement of the Terrestrial Mass 
Accretion Rate of Cosmic Dust," Science, Vol. 262, No. 5133, October 1993, pp. 550-553.

[26] Brownlee, D.E., "Cosmic Dust: Collection and Research," Annual Review of Earth Planetary Science, Vol. 13, 1985, pp. 147-173.

[27] NASA, "U.S. Standard Atmosphere 1976," NASA Technical Report, TM-X-74335, October 1976.

[28] Bauccio, M., "Physical Properties of the Elements," ASM Metals Reference Book, $3^{\text {rd }}$ Ed, ASM International, Materials Park, OH, 1993, pp. 142-147. 


\section{CHAPTER 5}

\section{A MILLIMETER-SCALE LORENTZ PROPELLED SPACECRAFT*}

\subsection{Abstract}

We evaluate Lorentz force actuation as a means of propellantless propulsion for millimeter-scale spacecraft by examining the acceleration and plasma-charging benefits associated with small length scales. A fully integrated "spacecraft on a chip" is described in terms of each of the traditional spacecraft subsystems, incorporating relevant research in microfabrication. Our candidate spacecraft design periodically pulses a RF beacon for ground-based orbit determination and powers a microchip payload. The spacecraft develops a net negative charge for Lorentz orbit augmentation by using solar power to differentially charge the surfaces of a sphere and filament. Having generated a multidimensional fit of plasma-sheath data generated through NASA's Charging Analyzer Program (NASCAP), we size and optimize these two charge-carrying geometries, accounting for plasma capacitance and power requirements. In a circular orbit at $350 \mathrm{~km}$, our design achieves a charge-to-mass ratio of $-2.5 \mu \mathrm{C} / \mathrm{kg}$ resulting in a daily deviation of $18 \mathrm{~m}$ from a Keplerian orbit. We conclude that the Lorentz force can indeed serve as a low-mass means of infinite specific-impulse propulsion for extremely small spacecraft.

\footnotetext{
* Reproduced with permission from the American Institute of Aeronautics and Astronautics (AIAA). Originally presented at the AIAA Guidance, Navigation, and Controls Conference, Hilton Head SC, Aug 20-23 2007 as paper number: 2007-6847.
} 


\subsection{Introduction}

System-level optimization is fundamental to successful spacecraft engineering. "Systems-on-chip" can accomplish the same tasks as a traditional system at a fraction

of the mass and cost. We explore the limits of spacecraft systems engineering by proposing the design and fabrication of an entire spacecraft's functions onto a single chip. This pursuit is motivated by a variety of unique advantages. Economically, reduced satellite mass and volume correspond directly to savings in rocket fuel. Further, the manufacturing processes associated with silicon technology allow for high-volume, low-cost production. Operationally, miniaturization may enable swarms of small satellites to perform large-scale distributed sensing with high redundancy. Physically, forces typically dismissed as slow-acting perturbations can be used as a source of infinite impulse at this scale. It is this last principle that has led us to study spacecraft miniaturization as a complement to our Lorentz Augmented Orbit research, which investigates an electromagnetic means of transferring energy and momentum to a spacecraft's orbit via a rotating planetary magnetic field.

It is interesting that miniaturization could be the key to demonstrating useful propellantless propulsion, when a significant portion of current research in the field focuses on the challenges associated with the extremely large structures necessitated by the characteristically weak perturbation forces. Kilometers-long tethers and square-kilometer-sized sails have been proposed as means of collecting sufficient impulse from the space environment for useful orbital adjustment.

The intent of this work is to create the first fully self-sustaining "Spacecraft-onChip" (SOC) capable of demonstrating observable propulsion via the Lorentz force. Inspired by the success of the first Sputnik launch in 1957, our design is intended to be as simple as possible. For three weeks, the small sphere of Sputnik I "beeped" down 
to Earth its internal temperature as it orbited overhead as the first demonstration of artificial satellites. A half century later, we attempt to repeat this achievement using less than a hundred-thousandth of its mass. Our design packages the traditional spacecraft subsystems (power, attitude control, communications, etc) onto a single silicon microchip capable of traveling in a non-Keplerian orbit. This paper outlines our general design methodology, describes the nonlinear dynamics, discusses applicable technologies, and proposes a candidate design.

\subsection{Mission Overview}

Our proof-of-concept goals suggest as simple a mission as possible. In order to validate Lorentz propulsion, we propose simultaneously launching two identical SOCs (or many pairs of identical SOCs), with one's Lorentz actuation disabled. Because the two spacecraft would be identical in every respect, differential drag and related effects would be minimized. Then, deviation of one's orbit from the other would be due

primarily to the Lorentz force. If the two systems are identical, this demonstration is single-fault tolerant at a high level. A four-phase operational sequence of events supports these measurements:

\subsubsection{Launch / Separation}

A circular LEO injection serves as the baseline launch. A likely deployment device would eject the SOCs from a parent spacecraft using adhesive that degrades in UV, a release of localized compressive load on the chip due to thermal expansion in sunlight, or perhaps a simple spring mechanism. Once separated from the upper stage or the primary satellite, the pair of SOC spacecraft would separate from one another in a way 
that minimizes unpredictable relative $\Delta \mathrm{V}$ (e.g. the degradable $\mathrm{UV}$ binder approach). Lorentz-induced perturbations of the Keplerian injection orbit are measured and provide the mission-demonstration data we seek. Section B discusses the range of orbits that can that can lead to useful measurements. This versatility, coupled with a robust, extremely low-mass design, enables a wide range of secondary launch opportunities.

\subsubsection{Charge / Maneuver}

After separation the spacecraft establishes an electrostatic charge such that it experiences the Lorentz force as it moves with respect to Earth's magnetic field. Among the various means of producing and holding this charge that this paper considers, our preferred architecture uses solar power and differential plasma-charging effects. Such architecture offers several benefits: it maximizes the total charge-tomass ratio, lends itself well to microscale fabrication, and requires no active means of expelling charged particles.

\subsubsection{Transmit / Sense}

The spacecraft communicate their position and temperature in a Sputnik-inspired fashion by periodically releasing a burst of electromagnetic energy. The frequency of these pulses is determined by the temperature of the on-board oscillator and the delay between pulses is determined by the available power. This design is one of many subsystem architectures that exploits unique benefits of the integrated circuit SOC concept. The ground segment of this project depends on appropriate ground stations to detect the beeps and infer range from data including acquisition-of-signal time, signal 
gain, and transmission frequency. The ranging data are assembled to reconstruct the orbit time history, demonstrating the effectiveness of the Lorentz propulsion by comparing the orbital element estimates of the uncharged SOC spacecraft to those of the charged one.

\subsubsection{End of Life}

Because of its propellantless architecture, an SOC spacecraft could continue to orbit and maneuver indefinitely without concern for station keeping propellant. Therefore, the end-of-life phase is characterized by the growth of distance between the two spacecraft to a point where the Lorentz force no longer constitutes most of the perturbation. Likely, radiation effects or thermal stress fatigue will degrade the SOC over time and will also limit the useful life.

\subsection{Lorentz Augmented Orbit Mechanics}

Our spacecraft design is driven by our proposed means of electrostatic propulsion. The spacecraft's Lorentz-augmented orbit (LAO) arises because a charged spacecraft traveling through a body's rotating magnetic field can transfer energy and momentum to and from the central body via the Lorentz force. This phenomenon is most clearly observed in the charged dust orbiting Jupiter, where Lorentz perturbations generate unique structures in the rings. Though thorough derivations have been presented elsewhere [1,2], we briefly overview the relevant concepts here.

Let $q$ represent the net biased electric charge and $\boldsymbol{v}_{\boldsymbol{r}}$ the velocity relative to a magnetic field $\boldsymbol{B}$. Then, the Lorentz force $\boldsymbol{F}_{\boldsymbol{L}}$ experienced by a charged spacecraft is

$$
\boldsymbol{F}_{L}=q \boldsymbol{v}_{r} \times \boldsymbol{B}
$$


For a rotating magnetic field

$$
v_{r}=\dot{r}-\omega_{B} \times r
$$

where $\dot{r}$ is the vector time derivative of the spacecraft's position $\boldsymbol{r}$ (with magnitude $r$ and direction $\hat{\boldsymbol{r}}$ ) relative to the system's barycenter in a Newtonian frame, and $\omega_{\boldsymbol{B}}$ is the angular velocity of the magnetic field. Based on a two-body Newtonian gravity model, the equation of motion for a charged orbiting spacecraft is

$$
m \ddot{\boldsymbol{r}}=-m \frac{\mu}{r^{2}} \hat{\boldsymbol{r}}+q\left(\dot{\boldsymbol{r}}-\boldsymbol{\omega}_{\boldsymbol{B}} \times \boldsymbol{r}\right) \times \boldsymbol{B},
$$

where $\mu=M G$, with parent body mass $M$ and universal gravitational constant $G$. Dividing through by the spacecraft mass $m$, we find that Lorentz acceleration is driven by the parameter $q / m$, the spacecraft's charge-to-mass ratio. The magnetic field at Earth is approximated by its dominant mode, a dipole with magnetic induction $B_{0}$ measured at a reference distance $r_{0}$ is

$$
\boldsymbol{B}=-B_{0}\left(\frac{r_{0}}{r}\right)^{3}(2 \cos \delta \hat{\boldsymbol{r}}+\sin \delta \hat{\boldsymbol{\delta}})
$$

expressed in right-handed spherical coordinates defined by radial $\hat{\boldsymbol{r}}$, azimuthal $\hat{\boldsymbol{\theta}}$, and polar $\hat{\boldsymbol{\delta}}$ directions. We simplify the field by aligning its spin with its parent body (neglecting the dipole tilt). Previous work has demonstrated that this simplification captures the governing physics with little loss of accuracy [2].

Though LAO has many potential applications, we focus our work on its simplest demonstration: non-Keplerian LAO effects in a circular orbit at the magnetic field's equator. Figure 5.1 indicates the directions of the Lorentz force vectors for a charged body in a prograde orbit subject to these conditions. Here, the directions of the central body's spin axis and magnetic field vector are defined by the right-hand rule. Earth's 
geographic North Pole corresponds to its magnetic south pole; hence the magnetic field's direction is shown as roughly opposite to its spin direction. The spacecraft's inertial and relative velocities lie perpendicular to its position vector and the magnetic field vector, constraining the Lorentz force's line of action along the radial direction $\hat{\boldsymbol{r}}$. These constraints imply that the Lorentz force cannot do work on the orbit $\left(\boldsymbol{F}_{\boldsymbol{L}} \cdot \dot{\boldsymbol{r}}=0\right)$ or alter its angular momentum $\left(\boldsymbol{r} \times \boldsymbol{F}_{L}=0\right)$. Therefore, the Lorentz force can alter only the spacecraft's potential energy, effectively changing the magnitude of gravity. The ratio of the magnitudes of Lorentz and gravitational accelerations is given by the dimensionless parameter $\beta$ :

$$
\beta=\frac{q}{m} \frac{B_{0}}{\mu} \frac{r_{0}^{3}}{r}\left[\sqrt{\frac{\mu}{r}} \pm \omega_{B} r\right],
$$

where the plus sign denotes a retrograde circular orbit, and the minus sign denotes a prograde circular orbit. For the sake of compatibility with secondary-launch opportunities, we focus on prograde orbits. Figure 5.2 plots the prograde acceleration ratio $\beta$ for a charged spacecraft orbiting Earth as a function of radial distance for a unit positive charge-to-mass ratio $(1 \mathrm{C} / \mathrm{kg})$. As illustrated in the figure, the Lorentz force approaches zero as the spacecraft approaches geostationary orbit, where the spacecraft's relative velocity approaches zero $\dot{\boldsymbol{r}}=\boldsymbol{\omega}_{B} \times \boldsymbol{r}$. In view of $\beta$ 's steep reduction with increasing altitude, we concentrate on LEO to maximize the perturbation. 


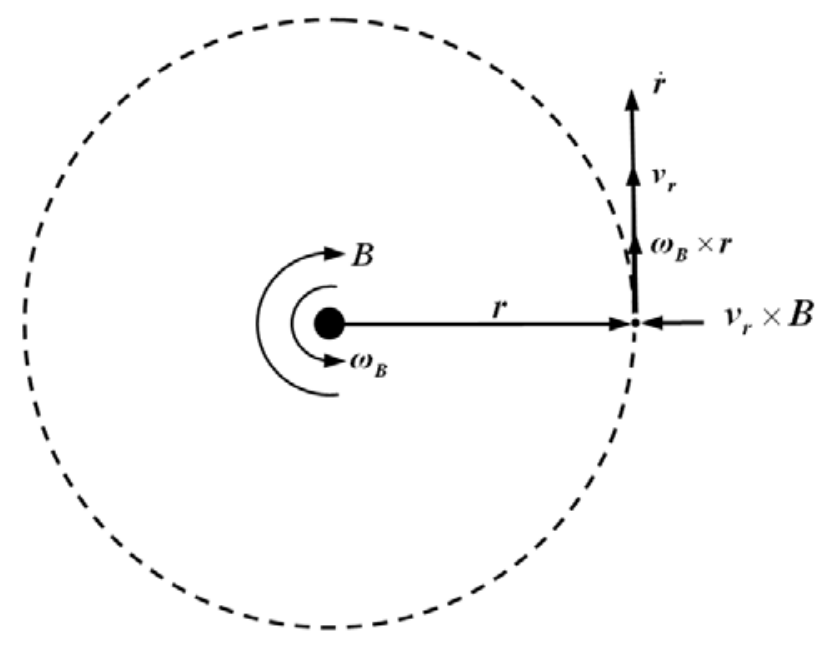

Figure 5.1. Vector depiction of the Lorentz force at various locations in a prograde, circular, magnetic-equatorial orbit.

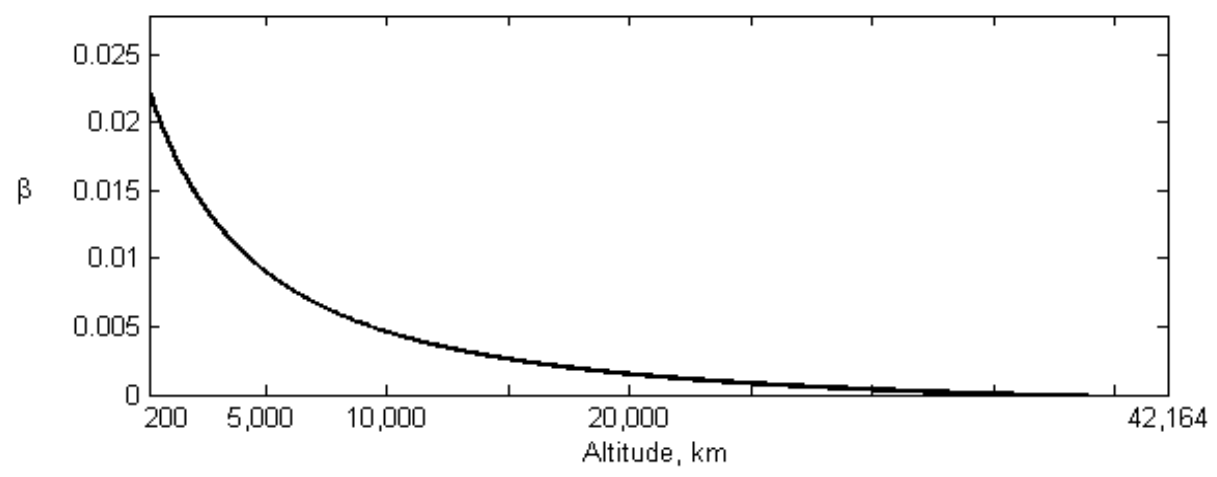

Figure 5.2. Magnitude of acceleration due to Lorentz force relative to gravitational acceleration for a charged body with a unit charge-to-mass ratio $(1 \mathrm{C} / \mathrm{kg})$ in a circular orbit from LEO to geostationary orbit.

From force balance in the radial direction, similar to Kepler's third law but augmented with the Lorentz effect, the following result relates the magnitude of a charged spacecraft's angular velocity $\omega$ to its orbital radius [1]:

$$
\frac{q}{m}\left(\omega-\omega_{B}\right) B_{0} r_{0}^{3}+\omega^{2} r^{3}=\mu
$$


In terms of radius as the dependent variable, the equation becomes

$$
r=\sqrt[3]{\frac{1}{\omega^{2}}\left(\mu-\frac{q}{m}\left(\omega-\omega_{B}\right) B_{0} r_{0}^{3}\right)}
$$

Alternatively, the angular velocity expressed in terms of radius yields two solutions

$$
\omega=\frac{-\frac{q}{m} B_{0} r_{0}^{3} \pm \sqrt{\left(\frac{q}{m} B_{0} r_{0}^{3}\right)^{2}+4 r^{3}\left(\mu+\frac{q}{m} \omega_{B} B_{0} r_{0}^{3}\right)}}{2 r^{3}},
$$

where addition is associated with a prograde orbit and subtraction with a retrograde orbit.

These results represent two LAO maneuvers that can be easily accomplished with a charged spacecraft. Figure 5.3a shows a circular formation that is impossible with purely Keplerian orbits: charged spacecraft with the same angular velocity as uncharged spacecraft at separate altitudes. Alternatively, Figure 5.3b illustrates a charged spacecraft in a circular orbit of the same radius as an uncharged spacecraft, but with a different angular velocity. For the second case, the along-track separation $\Delta \theta$ per day between the two spacecraft is evaluated with Earth conditions at an altitude of $350 \mathrm{~km}$ and plotted in Figure 5.4 on a log scale. For a prograde orbit, a positive charge-to-mass ratio causes the spacecraft to lag behind an uncharged spacecraft, and a negative charge-to-mass ratio leads an uncharged spacecraft. This figure suggests types of LAO-driven deviations from a Keplerian orbit that may be observable, even for relatively low charge-to-mass ratios. To avoid the subtle sequence of DV maneuvers necessary to create the formation in Figure 5.3a, our candidate mission is therefore to release a charged spacecraft-on-a-chip at about $350 \mathrm{~km}$ altitude and to measure its daily deviation from an uncharged orbit. 


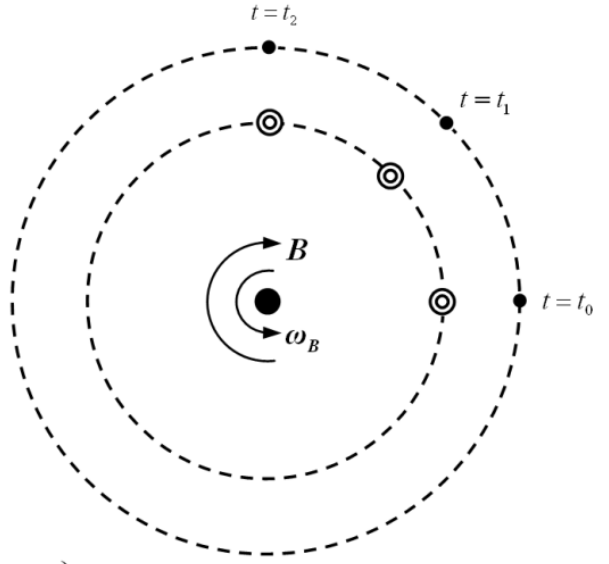

a.)

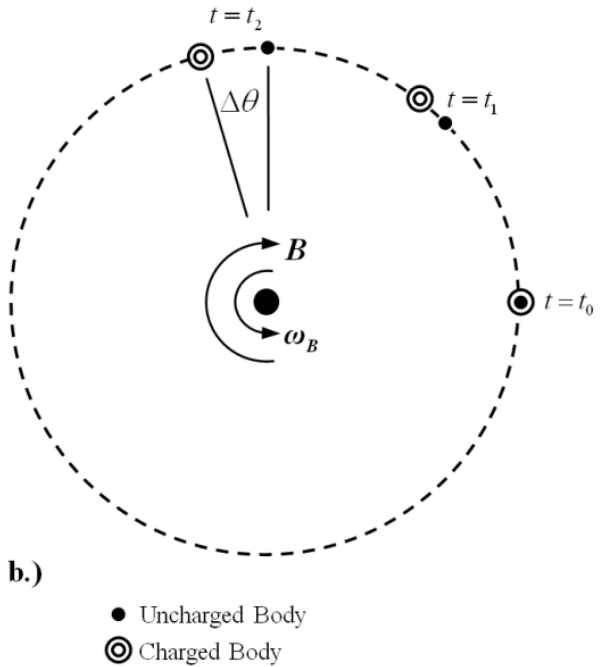

Figure 5.3. Deviations from Keplerian orbits by charged spacecraft in circular magnetic-equatorial orbits. a.) constant-radius formation flying and b.) along-track separation.

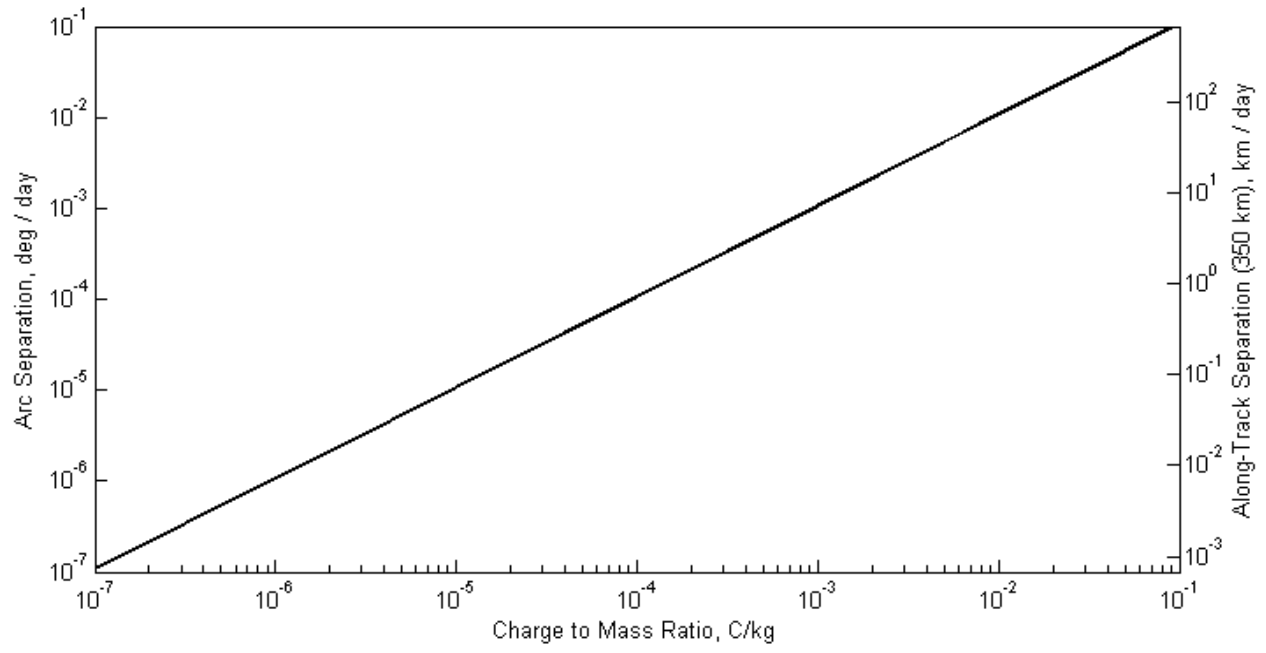

Figure 5.4. Arc separation rate between a charged and uncharged spacecraft in a prograde circular orbit at $350 \mathrm{~km}$ altitude.

\subsection{Space Plasma Effects}

Charge allows the spacecraft to interact with a planet's magnetic field and transfer energy from the planet's rotation into the spacecraft's orbit. In a perfect vacuum, orbital adjustment via the Lorentz Force is essentially power-free because the charge 
remains on the spacecraft indefinitely. However, charging interactions with the LEO plasma environment leads to some leakage of charge. This leakage rate, or plasma current, dictates the power requirement. However, maintaining this spacecraft charge is the only aspect of an LAO that demands power. Indeed, coupling between the Earth's magnetic field and plasma necessitate an optimization between the effectiveness of LAO and the power demands associated with the local plasma density. Though it is unreasonable to adequately discuss the physics of spacecraft plasma charging, we attempt to offer a cursory overview of the most relevant mechanisms.

A conductive body orbiting in a plasma attains a non-zero equilibrium potential $\varphi_{s}$, determined by the incident currents associated with processes such as local plasma attraction, photoelectric emissions, and artificial (e.g. electron-beam) currents. Accounting for a body's self-capacitance $C$ (roughly, its ability to hold charge on its surface), this equilibrium potential corresponds to an equilibrium charge:

$$
q=C \phi_{s}
$$

For a desired charge to be established, a biased potential must be generated on a body with sufficiently large self-capacitance. To maintain this potential, current must be provided to negate the natural charging mechanisms, motivating our research into spacecraft charging models. As for self-capacitance, our design focuses on the most well-understood charging geometries: a sphere and a thin wire.

The LEO environment is treated as a plasma consisting of an equal number-density of electrons and positive ions (i.e. a quasi-neutral plasma). An initially neutral body in a static quasi-neutral plasma will maintain a near-zero floating potential. However, LEO spacecraft traverse the local plasma with mesosonic velocities. That is to say, their velocity is much greater than the thermal velocity of the massive positive ions 
(primarily $\mathrm{O}^{+}$within our region of interest), but much less than the thermal velocity of electrons. For this reason the spacecraft interacts with the two polarities of particles differently.

Electron velocities exceed that of the spacecraft, meaning that electrons can all but instantaneously collect or evacuate a region around the spacecraft. As they approach a positively biased body, their charge cancels a portion of the electric field, reducing the net attraction experienced by other electrons. The result is a steady-state electron distribution around the charged body known as a plasma sheath. A sample plasma potential profile associated with a sheath is illustrated in Figure 5.5. The potential of the plasma decreases with distance from the body until it reaches the quasi-neutral plasma potential at its outer sheath edge, the point at which the electric field associated with the body is cancelled (or shielded) by the distribution of oppositely charged particles around it.

The current associated with these electrons is defined as the electron thermal current

$$
I_{t h}=n q_{e} A_{S O} \sqrt{\frac{T}{2 \pi m_{e}}}
$$

where $n$ is the unperturbed electron number density, $q_{e}$ is the charge of an electron, $A_{S O}$ is the surface area defined by the outer sheath edge, $T$ is the quasi-neutral temperature of the plasma reported in $\mathrm{eV}$, and $m_{e}$ is the mass of an electron. Though not explicitly shown, thermal current has a strong dependence on the body's potential through the sheath surface area which will be discussed below.

With respect to positive ions, LEO spacecraft velocities are supersonic. Positive ions within the outer sheath edge are attracted to the negatively charged body, but are unable to react quickly enough to collect around the body. Only the ions that 
are directly impacted by the area enclosed by the outer sheath edge can react with the spacecraft. Of these, a portion $\eta_{\text {ram }}$ contact the spacecraft and impart their charge. This mechanism is known as ram current

$$
I_{\text {ram }}=\eta_{\text {ram }} n q_{i} v_{r} A_{\text {ram }}
$$

where $n$ is the unperturbed ion number density, and $q_{i}$ is the charge of the ion species. $A_{\text {ram }}$ is related to the frontal area (normal to $\boldsymbol{v}_{\boldsymbol{r}}$ ) of the spacecraft's outer sheath edge [3]. A ram efficiency of 0.7 has been suggested as an estimate [4], which we adopt here. The inability of positive ions to collect around a negatively charged spacecraft generates a region with an absence of elections. That is to say, electrons are repelled from the spacecraft, while positive ion densities remain essentially unaffected. The result is a positive charge distribution surrounding the spacecraft comparable to Figure 5.5 .

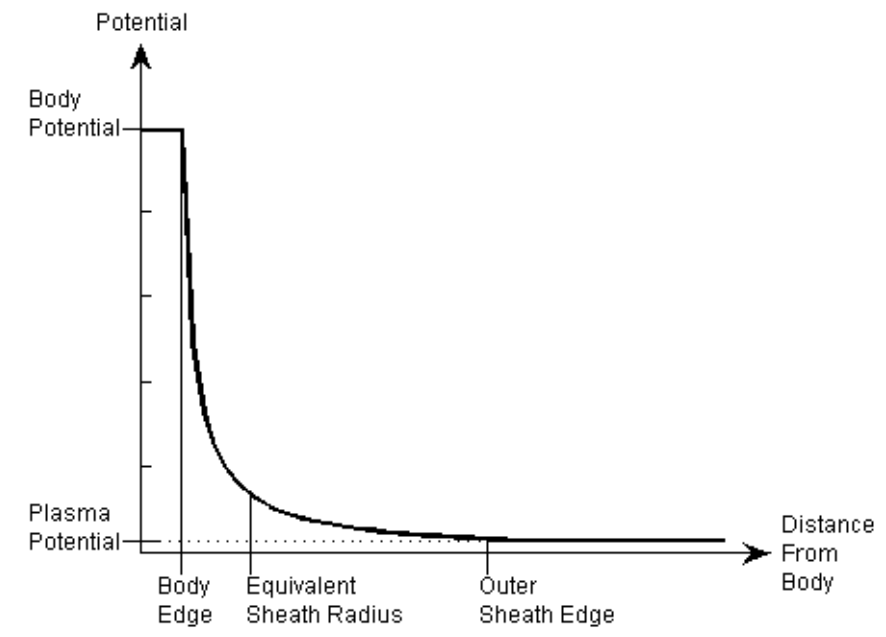

Figure 5.5. Illustration of plasma potential as a function of distance from the surface of a spacecraft. 


\subsubsection{Sphere Charging}

For a sphere, the potential distribution outside the body (as depicted in Figure $5.5)$ is

$$
\phi(x)=\phi_{s} \frac{x}{r_{s}} \exp \left(\frac{-\left(x-r_{s}\right)}{\lambda_{D}}\right),
$$

where $\varphi_{s}$ is the potential of the sphere, $r_{s}$ is the radius of the sphere, and $x$ is the distance from the surface of the sphere [5]. The coefficient in the exponent is a characteristic dimension of the plasma, the Debye length

$$
\lambda_{D}=\sqrt{\frac{\varepsilon_{0} T}{n q_{e}^{2}}}
$$

where $\varepsilon_{0}$ is the permittivity of free space. For low sphere potentials, the effective ram area is defined by a circle with a radius of a few Debye lengths. However, as the body's potential increases, the outer sheath edge increases as well. A survey of space plasma literature resulted in two models used to estimate the outer sheath edge for a charged sphere.

The first model used to predict this increased outer sheath size $S_{O}$ for a sphere with a high potential in a plasma environment is the Child-Langmuir Law

$$
S_{O}=\frac{\sqrt{2}}{3} \lambda_{D}\left(\frac{2 \phi_{s}}{T}\right)^{\left(\frac{3}{4}\right)}
$$

The second model, by Blackwell et al, consists of an analytic fit to the numerical results of Laframboise, incorporating the sphere's radius [6,7]

$$
S_{O}=\left(2.5-1.87 e^{-0.39 \frac{r_{s}}{\lambda_{D}}}\right) \lambda_{D}\left(\frac{\phi_{s}}{T}\right)^{\left(\frac{2}{5}\right)} .
$$


The differences in thermal and ram charging mechanisms suggest that power requirements are affected by the polarity of the charge on the body as well as the magnitude. A comparison of Eqs 10 and 11 indicates that the thermal current associated with a positively charged body exceeds the ram current for an oppositely charged body for

$$
v_{r}<\sqrt{\frac{8 \pi T}{m_{e}}}
$$

Indeed, LEO plasma satisfies this condition, and sample values suggest that a positively charged body requires as much as a hundred times more power than a negatively charged body.

A second pertinent sheath parameter is the equivalent sheath radius. The oppositely charged body of particles (or lack thereof, as discussed for a negatively charged body), forms a concentric shell that increases the available self-capacitance of the spacecraft. That is, the capacitance of a conductive sphere is

$$
C_{s}=4 \pi \varepsilon_{0} r_{s},
$$

while two oppositely charged concentric spheres separated by a distance $S_{E}$ give

$$
C_{S I}=4 \pi \varepsilon_{0} r_{s} \frac{\left(r_{s}+S_{E}\right)}{S_{E}} .
$$

This approximation treats the surrounding plasma's total charge as a single spherical shell at an equivalent distance. This distance is indicated in Figure 5.5 and marks the area centroid of the potential profile. A small equivalent sheath radius can significantly increase a body's self-capacitance and therefore its charge. 
Noting that the two models discussed above (Eqs $14-15)$ estimate the outer sheath edge only, and are based on a relatively limited range of potentials, we turn to NASA's Charging Analyzer Program (NASCAP) for further insight.

NASCAP is an industry-standard finite-element modeling toolkit that simulates the spacecraft and plasma interactions spatially and temporally. After validating our method with a $40 \mathrm{kV}, 1 \mathrm{~m}$ radius conductive sphere studied by Parker [8], we evaluated a range sphere sizes and potentials in a static LEO environment, with the constants given in Table 5.1. A sample of the graphical results from a NASCAP simulation for a $1 \mathrm{~cm}$ sphere held at $10 \mathrm{kV}$ potential is shown in Figure 5.6.

\begin{tabular}{lc} 
Table 5.1. NASCAP Simulation Parameters \\
\hline \hline Environment & LEO \\
Space Charge Model & Analytic, Non-Linear \\
Average RMS Error, V & $1 \mathrm{E}-3$ \\
Plasma Density, $\mathrm{m}^{-3}$ & $1 \mathrm{E} 12$ \\
Plasma Temperature, eV & 0.10 \\
Corresponding Debye Length, $\mathrm{m}$ & $2.351 \mathrm{E}-3$ \\
Sphere Material & Aluminum \\
Sphere Approximation & 80 Element Triangle Mesh \\
\hline \hline
\end{tabular}

Each simulation produces a radial plasma potential distribution around the sphere. The outer sheath edge can be found by calculating of the distance at which this potential is reduced to within the error tolerance of the solver. Next, the potential distribution around the sphere is fit to a curve of the theoretically likely form

$$
\phi(x)=a_{0} \frac{r_{s}}{x} \exp \left[a_{1}\left(x-r_{s}\right)\right]
$$

by solving for the unknown coefficients $a_{0}$ and $a_{1}$. This function, reminiscent of Eq 5.12 and Figure 5.5, is used to find the spatial charge density $\boldsymbol{\rho}$ via Poisson's equation 


$$
\nabla^{2} \boldsymbol{\varphi}=\frac{-\boldsymbol{\rho}}{\varepsilon_{0}}
$$

Assuming spherical symmetry and integrating radially from the body's surface to infinity yields the net charge contained in the local plasma

$$
q=-\varepsilon_{0} \int_{V} \nabla^{2} \varphi d V=-4 \pi \varepsilon_{0} a_{0} r_{s}\left(1-a_{1} r_{s}\right)
$$

This charge, equal and opposite to the charge contained on the sphere, allows us to estimate the improvement to the sphere's capacitance due to the presence of the local plasma.
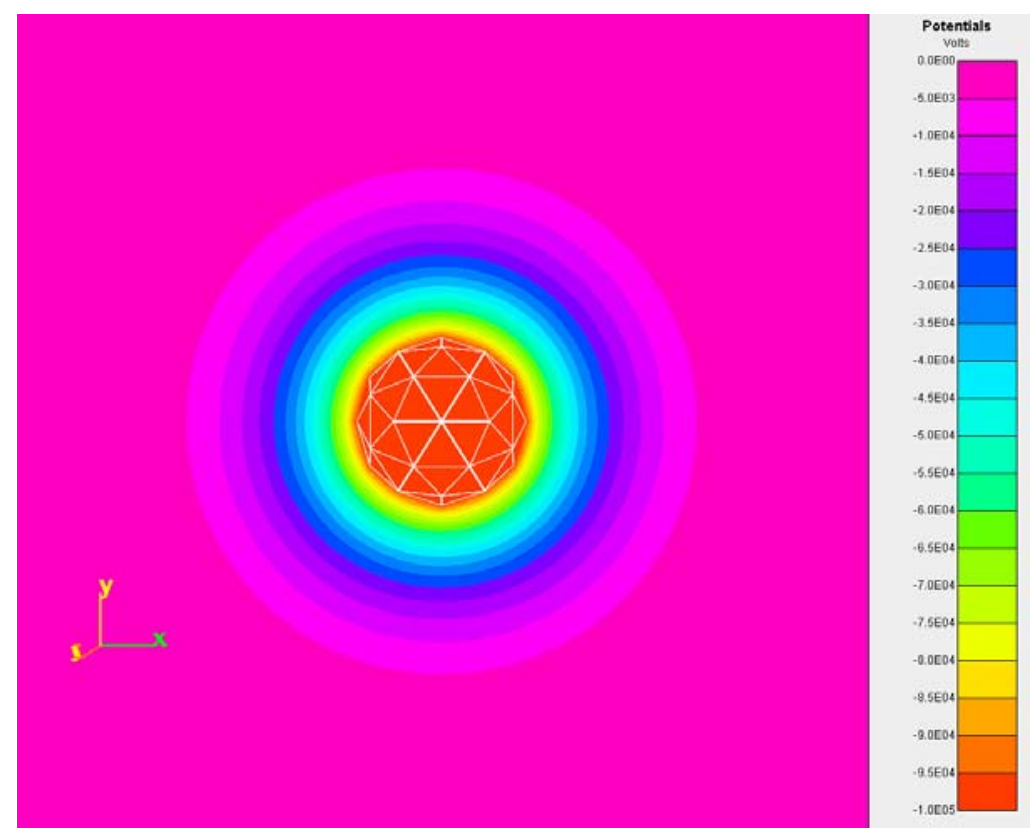

Figure 5.6. Sample NASCAP static plasma potential results for a sphere model charged to $-10 \mathrm{kV}$ potential at $350 \mathrm{~km}$.

Table 5.2 gives the inner and outer sheath sizes calculated from these simulations. Five sphere sizes were evaluated at potential from $100 \mathrm{~V}$ to $5.3 \mathrm{MV}$, the potential of the alpha particles emitted from radioactive polonium-210 discussed at a later point in this paper. At small size scales $\left(r_{s}=0.01 \mathrm{~m}\right.$ and $\left.0.1 \mathrm{~m}\right)$ and high potentials $\left(\varphi_{s}>100 \mathrm{kV}\right)$, 
the equivalent sheath merges with the outer sheath as a result of the different methods of calculating these two parameters. The implication is that the equivalent sheath is no longer effective for adding useful capacitance beyond that potential. At large size scales $\left(r_{s}=10 \mathrm{~m}\right.$ and $\left.100 \mathrm{~m}\right)$ and low potentials $\left(\varphi_{s}<100 \mathrm{~V}, \varphi_{s}<1 \mathrm{kV}\right)$, NASCAP's grid format limited our ability to adequately resolve the potential profile.

Table 5.2. NASCAP Simulation Results (Equivalent Sheath in meters / Outer Sheath in meters)

\begin{tabular}{cccccc}
\hline \hline & \multicolumn{5}{c}{ Radius of Sphere, m } \\
\cline { 2 - 6 }$\varphi_{s}, \mathrm{kV}$ & 0.01 & 0.1 & 1 & 10 & 100 \\
\hline 0.1 & $0.04 / 0.09$ & $0.12 / 0.22$ & $0.31 / 0.60$ & - & - \\
1 & $0.10 / 0.25$ & $0.28 / 0.60$ & $0.79 / 1.84$ & $1.51 / 3.46$ & - \\
10 & $0.45 / 0.66$ & $0.60 / 1.88$ & $2.12 / 4.99$ & $3.61 / 9.74$ & $8.06 / 18.08$ \\
100 & 1.86 & 4.85 & $5.87 / 13.27$ & $13.77 / 29.56$ & $22.00 / 60.59$ \\
500 & 3.24 & 9.82 & $11.10 / 24.05$ & $32.65 / 66.20$ & $66.83 / 186.13$ \\
& & & & $117.76 /$ & $228.79 /$ \\
5,300 & 9.98 & 23.2 & $32.65 / 72.67$ & 133.06 & 556.88 \\
\hline \hline
\end{tabular}

It should be emphasized that these simulations and their corresponding results are intended to describe the plasma parameter-space in an engineering sense, not to rigorously quantify or describe the associated physics. These values were then nondimensionalized and fit to second-order logarithmic polynomial surfaces. For a given LEO plasma temperature $T$ in $\mathrm{eV}$ and number density $n$ in $\mathrm{m}^{-3}$, the base-ten logarithm of the non-dimensionalized sphere radius and potential,

$$
\lambda_{r}=\log _{10}\left(\frac{r_{s}}{\lambda_{D}}\right), \lambda_{\phi}=\log _{10}\left(\frac{\phi_{s}}{T}\right)
$$

can be used to estimate the base-ten logarithm of the non-dimensionalized equivalent and outer sheath sizes,

$$
\lambda_{E}=\log _{10}\left(\frac{S_{E}}{\lambda_{D}}\right), \lambda_{O}=\log _{10}\left(\frac{S_{O}}{\lambda_{D}}\right)
$$


according to the second order polynomial surface-fit

$$
\lambda_{E, O}=c_{1} \lambda_{r}^{2}+c_{2} \lambda_{r} \lambda_{\phi}+c_{3} \lambda_{r}+c_{4} \lambda_{\phi}^{2}+c_{5} \lambda_{\phi}+c_{6}
$$

with coefficients given in Table 5.3 below.

Table 5.3. Coefficients for Surface Fits Given by Eq. 5.22

\begin{tabular}{lcc}
\hline \hline & Equivalent Sheath & Outer Sheath \\
\hline$c_{1}$ & -0.007934 & -0.014351 \\
$c_{2}$ & -0.010351 & 0.017511 \\
$c_{3}$ & 0.425960 & 0.367490 \\
$c_{4}$ & 0.009198 & -0.003839 \\
$c_{5}$ & 0.419800 & 0.446000 \\
$c_{6}$ & -0.311750 & 0.020039 \\
\hline
\end{tabular}

\subsubsection{Filament Charging}

For a filament, the charging mechanisms are closely related. As in the case of a sphere, a charged filament attracts or repels electrons such that a sheath develops. The outer edge of this sheath represents the distance at which the electric field of the filament is negated by the surrounding plasma's distribution. The equivalent sheath acts as a concentric cylindrical shell of opposite charge, increasing the geometry's capacitance according to

$$
C_{f}=\frac{2 \pi \varepsilon_{0} L_{f}}{\ln \left(\frac{r_{f}+S_{I}}{r_{f}}\right)}
$$

where $L_{f}$ is the length of a filament with radius $r_{f}$. Choinière gives models for estimating the equivalent sheath radius based on numerical and experimental work [9]:

$$
2.554\left(\frac{S_{I}}{\lambda_{D}}\right)^{1.325} \ln \left(\frac{S_{I}}{r_{f}}\right)=-\frac{\phi_{s}}{T},
$$




$$
S_{I}=0.123 \lambda_{D}\left(\frac{|q|}{L_{f}} \frac{1}{\varepsilon_{0} T}\right)^{0.755}
$$

This model is valid for potentials and filament radii that satisfy the two conditions:

$$
\begin{gathered}
r_{f}<0.1 \lambda_{D} \\
\phi_{s}>\frac{200}{2 \pi} \ln \left(\frac{S_{I}}{r_{f}}\right) .
\end{gathered}
$$

Power collection for a filament differs from that of a sphere. Charged bodies with characteristic lengths (the radius for spheres and cylinders) smaller than the local Debye length, orbit-motion-limited (OML) current collection dictates the power requirements:

$$
I_{O M L}=\frac{n q_{e} A_{f}}{\pi} \sqrt{\frac{2 q_{e}\left|\phi_{s}\right|}{m_{i, e}}},
$$

where $A_{f}$ is the total surface area of the filament and $m_{i, e}$ is the mass of the ions or electrons attracted by the filament's charge. For positively charged filaments, OML dictates the most efficient electron collection current. Alternatively, OML's mass dependence implies that a negatively charged filament in LEO will collect fewer massive ions than ram charging would predict.

Figure 5.7 gives a plot of the linear charge density and sheath size subject to these bounds for a $10 \mu \mathrm{m}$ radius filament, though it should be noted that the sheath is only very weakly dependent on the filament radius (as dictated by the bounds). The filament radius dictates a minimum potential of $300 \mathrm{~V}$ to satisfy the conditions of applicability. 


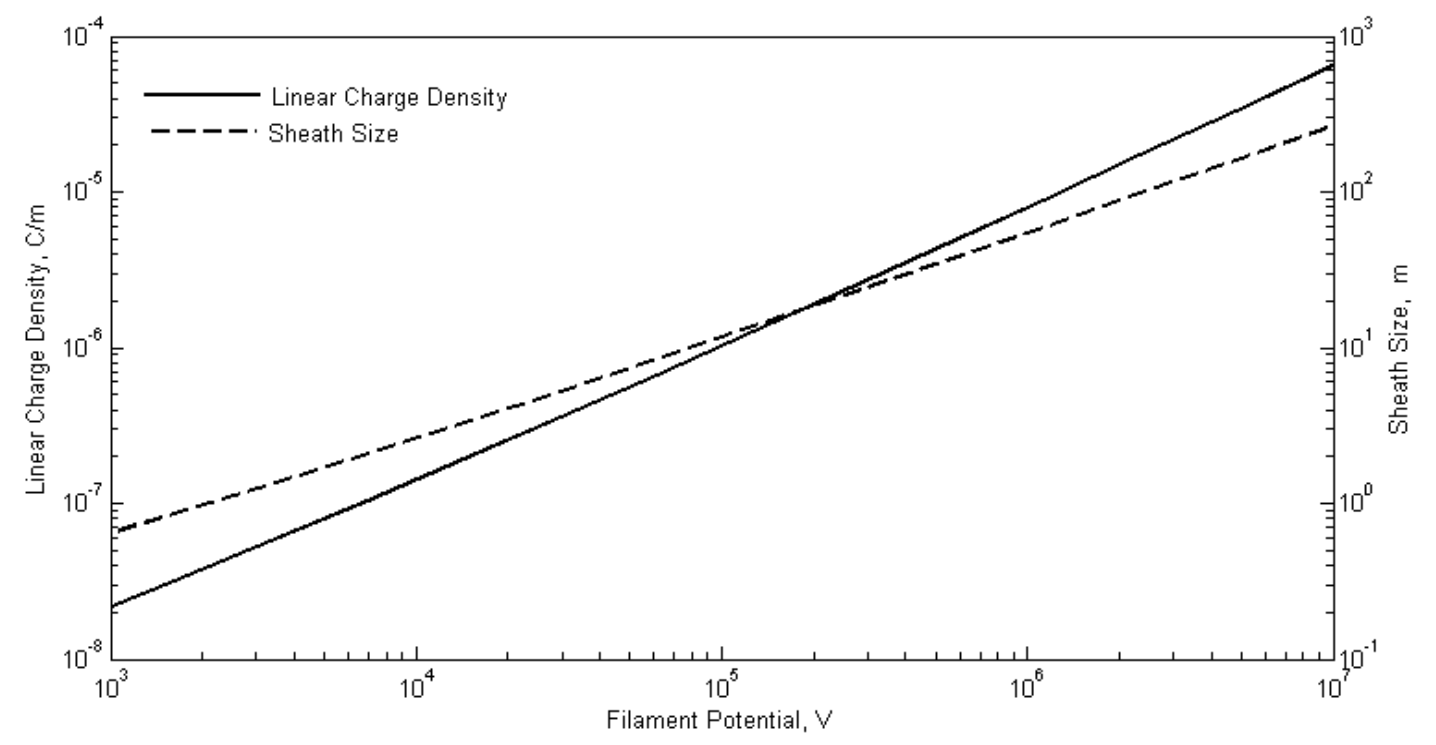

Figure 5.7. Linear charge density and sheath size as functions of negative potential potential for a $10 \mu \mathrm{m}$ radius filament.

\subsubsection{Differential Charging}

Geometry and polarity can result in different plasma-charging mechanisms. We exploit this difference to enable a net spacecraft charge using a potential source as proposed by Hoyt and Minor [4]. This method offers useful and controllable charge, as well as the advantage of requiring only a sufficient potential source.

In a vacuum, if two conductive spheres are connected to the terminals of a perfect battery, each sphere reaches a potential equal to half of the battery's potential and with opposing polarities. However, in a plasma environment, the spheres' opposite polarities generate dissimilar currents, resulting in dissimilar sphere potentials [3]. At LEO where thermal charging exceeds ram charging, the positive sphere's (henceforth referred to as the cathode) equilibrium potential can decrease almost to zero. The perfect battery continues to maintain the potential difference between the two spheres, 
requiring that the negative sphere's (anode) equilibrium potential consequently increase. The system then may then act as a single body with a net negative charge.

This system is somewhat inefficient, in that a portion of the net charge is reduced by the cathode's nonzero equilibrium potential. However, we can improve efficiency by varying the cathode's geometry. The goal is for the capacitance of the anode to far exceed the cathode so that at the equilibrium potential, the net charge on the system is decidedly negative. For example, one end can be a large sphere and the other a thin filament. Since OML is the most efficient current collection method, this latter example represents a mass-efficient charging architecture.

\subsubsection{Alternative Charging Concepts}

The advantages and capabilities of the differential-charge architecture discussed above makes it a clear candidate for our millimeter-scale spacecraft. However, this solution came about after exploring a number of possible charging mechanisms. For completeness, we briefly outline them here.

\subsubsection{Passive Charging}

Once inserted into an orbit, the chip experiences currents due to ram charging, thermal attraction, photoemission, and secondary interactions. Differences in these mechanisms cause a spacecraft to generally achieve a non-zero potential. For a sufficiently high ratio of capacitance to mass, these potential variations could prospectively perturb an orbit in a measurable manner, demonstrating LAO. Such is the case for dust in Jupiter's orbit, after all. A body in LEO typically attains an equilibrium potential of a few volts positive [5], though the plasma environment and thus charging conditions vary significantly both temporally and spatially. It is 
conceivable that a spacecraft could achieve novel resonances (again, as observed in dust at Jupiter). However, passive charging seems less desirable than other options because LEO equilibrium potentials are generally too low. Furthermore, the uncontrollable and unpredictable nature of this potential severely limits applications.

\subsubsection{Photoemission Charging}

One of the natural currents a spacecraft experiences is photoemission charging. Current is generated when a photon impacts a spacecraft's surface and liberates an electron. A continuous ejection of electrons represents a current that depends on the energy of the impacting photon (i.e. its frequency), the specific material properties (photoemission yield, surface treatment), and the angle of incidence. Currents associated with photoemission are on the order of $10^{-9} \mathrm{~A} / \mathrm{cm}^{2}[5]$. In an effort to maximize this current, a thin film of high-yield material could be deposited onto the surface of the charge geometry. Similar to passive charging, one can conceive of resonances incorporating the periodic solar eclipse or even forced by applying materials of different yields to different sides of the spacecraft.

Kasha calculates the floating potential to be $0.6 \mathrm{~V}$ for an aluminum $1 \mathrm{~m}$ radius sphere in a circular orbit at $350 \mathrm{~km}$ experiencing photoemission charging [10]. Based

on this value, it seems unlikely that photoemission current can establish charge-tomass ratios sufficient to demonstrate observable orbit modification, let alone serve as a useful means of propulsion.

\subsubsection{Radioactive Emission}

Another charging concept attempts to use radioactive emission as a current source. As radioisotopes naturally decay, they emit energetic particles. In the case of alpha 
decay, these particles are helium nuclei with a +2 charge. For beta decay, electrons or positrons are emitted with charge -1 or +1 respectively. These emissions represent a current which would alter the floating potential of a body [5]. Research on SPEAR suggests that the potential can reach the emitted particle's energy given sufficient current. This implies that a spacecraft could achieve a potential on the order of megavolts if the current is sufficient to overcome that of the incident plasma.

This method is not quite propellantless, in that it requires the launch of an initial mass of radioisotope that depletes itself over time. Only the particles that are ejected in a direction away from spacecraft contribute to the body's potential; the others remain within the isotope and dissipate their emission energy as heat. This principle suggests that current efficiency can be maximized if the isotope is deposited in a thin film onto the outer surface of the body. This current might also be modulated by altering the surface geometry or exposure. Note that this calculation uses the emission of particles to impart a charge, not momentum.

The following brief analysis of radioactive emissions as a means of thrust shows that significant orbital perturbations are not feasible. A sample of radioactive material decays exponentially according to:

$$
N(t)=N_{0} \exp \left(\frac{-t}{\tau}\right)
$$

where $N$ is the number of atoms in the sample with initial value $N_{o}$ at time $t=0$, and $\tau$ is the isotope's mean life given in units of time. The decay current is determined by the product of the decay rate, the charge of the emitted particles $q_{i s o}$, and an efficiency term $\eta_{\text {iso }}$ that accounts for particles unable to escape the spacecraft. Using the isotope's density $\rho_{\text {iso }}$ (with units atoms $/ \mathrm{kg}$ ), we find an expression for decay current

$$
I_{\text {iso }}=\eta_{\text {iso }} q_{\text {iso }} \cdot \frac{d N(t)}{d t}=-\eta_{\text {iso }} \frac{q_{\text {iso }} \rho_{\text {iso }} m_{0 i s o}}{\tau} \exp \left(\frac{-t}{\tau}\right) .
$$


Equating this current to the incident ram current allows us to solve for the spacecraft's floating potential over time. The results of these calculations for Polonium-210, a highly energetic alpha emitter $(5.3 \mathrm{MeV})$, applied to a centimeter radius sphere, after one year's decay, are shown in Figure 5.8. It is clear from these figures that the polonium is incapable of supplying enough current to attain charge-to-mass ratios sufficient for useful LAO maneuvers. The current associated additional radioactive material is not large enough to augment the total charge-to-mass ratio.

a.)

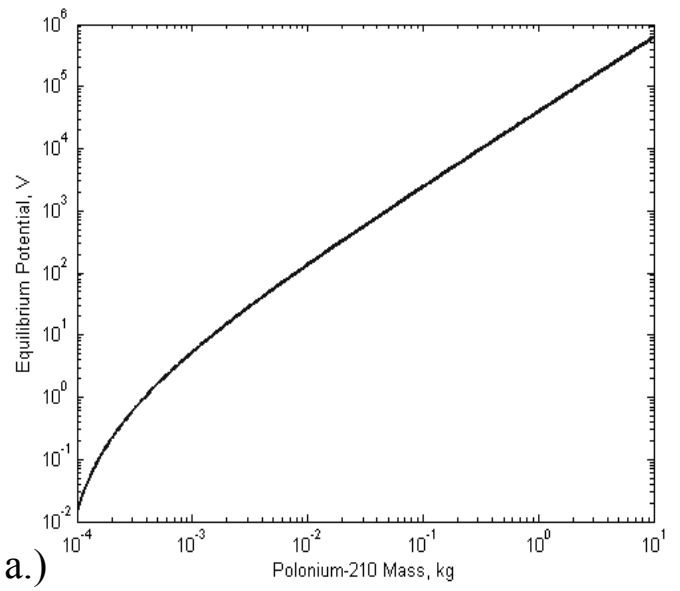

b.)

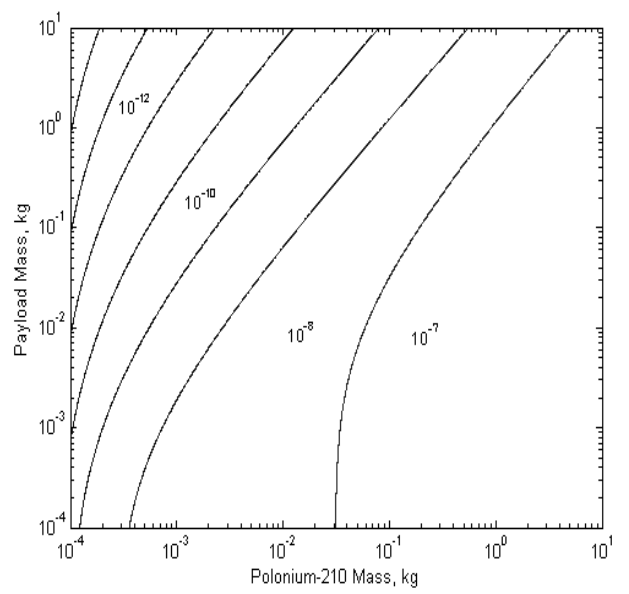

Figure 5.8. a.) Equilibrium potential in $\mathrm{V}$ as a function of polonium-210 mass and $\mathrm{b}$.) Charge-to-mass ratio in $\mathrm{C} / \mathrm{kg}$ as a function of polonium-210 mass and payload mass for a $1 \mathrm{~cm}$ radius sphere after $1 \mathrm{yr}$ at $350 \mathrm{~km}$.

\subsubsection{Pyroelectric Emission}

Yet another means of emitting charge from a body is the use of pyroelectric crystals. When subjected to changes in temperature, pyroelectric crystals rapidly polarize, generating intense electric fields. These fields can accelerate ions or electrons in a uniform beam, with electron energies on the order of $100 \mathrm{keV}$. If the temperature reverses, the electric field also reverses, attracting the opposite charge [11]. 
Differences in particle mobility could be instrumental in establishing a biased charge for rapid thermal cycles. That is, the current of ions attracted to the negative induced field is less than the electron current when the field reverses. An alternating current applied to a thermocouple could potentially generate extremely high potentials. Passively, the small thermal mass and large temperature gradients associated with solar eclipses could feasibly enable unique resonant orbits. Having observed these peculiar crystals firsthand, the possibility of incorporating them into a design is attractive. However, at present this technology is too immature to be incorporated in our mass estimates with any confidence.

\subsection{Spacecraft Architecture}

We approach the design space with an emphasis on simplicity, feasibility, and scalability. Our goal is to accomplish something unconventional using conventional technologies. The final product is a single densely populated microchip that incorporates the eight traditional spacecraft subsystems: propulsion, power, telecommunications, attitude determination and control, structure, and thermal control.

\subsubsection{Power}

Solar-cell power generation is both passive and semiconductor based, making it a natural selection for power supply. We focus on silicon-based first-generation solar cells, the most well researched and fabricated photovoltaics. These cells use a single layer $\mathrm{p}-\mathrm{n}$ junction diode to pass photovoltaic currents. These junctions lend themselves well to mass production silicon techniques with high volume-efficiency. By strategically connecting sets of individual cells in parallel or series, an array can be designed with specific voltage and current characteristics to accommodate LAO or 
payload requirements. Commercially, high efficiency cells commonly achieve specific power on the order of $200 \mathrm{~W} / \mathrm{kg}$. Presuming inefficiencies associated with integration in a MEMS environment, we conservatively model specific power as 100 $\mathrm{W} / \mathrm{kg}$. The solar array powers the charging mechanism. Thus, when the spacecraft is in eclipse, its charge will return to the local plasma potential, and the spacecraft orbit becomes temporarily Keplerian. In our final design, we calculate our solar array requirements for a spacecraft oriented at $45^{\circ}$ from the sun vector.

\subsubsection{Propulsion}

With few exceptions [12], traditional propellant mechanisms such as chemical or ion thrust subsystems are unreasonable at extremely small scales, and hence propulsion discourages extreme spacecraft miniaturization. We contend that the accelerations typically classified as perturbations, namely the Lorentz force, can offer useful thrust with relatively little hardware-overhead when incorporated into our microchip architecture. Here we explore and evaluate four capacitive structures to be considered for use as the conductive geometries that maintain non-zero equilibrium potential.

\subsubsection{Spherical Shell}

Aside from being the most well understood geometry regarding plasma interactions, a spherical shell architecture offers noteworthy design benefits. First, it's a natural choice because it distributes charge evenly over the surface, preventing charge loss associated with sharp-point field concentrations, and making efficient use of the available surface area. Second, the sphere can act as a Faraday cage, protecting the spacecraft within from external electric fields. 
Solar sail material research suggests that a likely shell candidate is CP1 polyimide film. It offers favorable mechanical properties with extremely low mass, as well as proven resistance to high thermal loads and radiation doses [13]. Sheets can be manufactured as thin as $5 \mu \mathrm{m}$, yielding an area density of $0.009 \mathrm{~kg} / \mathrm{m}^{2}$. The spacecraft could be sealed within the deflated shell at launch, and packed efficiently into a release mechanism. At the charge phase of the mission, the shell would then inflate itself to full size via electron repulsion on its surface. A transparent version, perhaps kapton coated with ITO for conductivity, can be used for solar-power collection through the sphere.

\subsubsection{Filament}

An extremely thin filament can efficiently store charge along its length and in the large volume of its sheath. The Lorentz force, acting along the filament, tends to pull the filament and thus the spacecraft along its direction. The Lorentz force will facilitate deployment and stabilizes its dynamics, a challenging issue associated with tethers in space. This effect can also be used to constrain the spacecraft attitude in two of the three attitude degrees of freedom.

Though the prospects of long tethers seem challenging, it should be noted that the TSS-1R electrodynamic tether mission successfully deployed a $20.7 \mathrm{~km}$ filament in LEO [14]. We consider commercially available $10 \mu \mathrm{m}$ radius aluminum filaments with a linear density of $0.848 \mathrm{mg} / \mathrm{m}$.

\subsubsection{Collection of Filaments}

This concept considers charge storage on a group of filaments bound together

to form a sparse structure. One can conceive of a spherical or cylindrical capacitor 
built as a mesh of wires, rather than a single solid shell. Some research suggests that filaments grouped with a separation distance much smaller than the outer sheath edge, behave comparably to single solid-shell body [9].

This architecture offers the advantages of a spherical geometry with a significant savings in mass. The filaments form a Faraday cage that self-inflates with charge. Further, the spacecraft within the cage has superior access to solar power and telecommunications than would a spacecraft within a solid shell. Simple calculations indicate that less than $5 \%$ of the chip's surface might be shadowed by a filament structure. Like the sphere, the mesh can be compacted for launch and then passively expanded by charging.

\subsubsection{Electrets}

For the sake of completeness, we reference a means of volumetric charge storage that we have explored but choose not to incorporate into our design: electrets. Electrets are insulators that are artificially implanted with ions to create a quasipermanent biased charge. The ions are mechanically bound within the material, creating an electric field.

Applications to integrated circuits have motivated research into silicon-based

electrets. Thin films of insulating material are bombarded with high energy ions to yield high charge-to-mass ratios. Teflon variants, for example, suggest charge to mass ratios on the order of $10 \mathrm{mC} / \mathrm{kg}$ [15]. Concern with electrets stems from their charge lifetime. In laboratory applications, electrets typically maintain their charge integrity on the order of years. However, it remains to evaluate this timeframe in the space plasma environment. Presumably, high energy particle bombardment would reduce it 
beyond a useful mission life. However, these materials have yet to be evaluated fully for their application to LAO spacecraft.

\subsubsection{Solution Space}

Among these four architectures, a spherically shaped mesh anode and a filament cathode offer an effective architecture in terms of mass efficiency, charge density, and ease of system integration. The appropriate charging model for the "mesh" anode is the source of some uncertainty. It stands to reason that the filament separation distance dictates whether the sphere is "seen" by the plasma as a single entity experiencing sheath-related ram charging, or as a set of filaments individually charging according to OML theory. With this in mind, we selected the Debye length as the separation distance, evaluated both methods, and count on the more conservative of the two results.

With an architecture selected, we find that the details of the design are dictated by the plasma conditions, anode size and potential, and chip mass. Figure 5.9 shows the solution flow-diagram for a system analysis using ram-charging as the defining current. Likewise, Figure 5.10 gives the solution flow-diagram for the OML charging model of the mesh sphere anode. The important resulting design parameters are given in bold outline. In this flow, the anode architecture is used to determine the value of the dominant ion current. A simple current balance demonstrates that this current, though driven by the solar cell array, is supplied by the cathode. The length of the filament is then found by setting the ion current equal to the cathode filament's OML current. This enables the differential charging and leads to the final charge-to-mass ratio. 
Both ram and OML charging were incorporated into models and used to evaluate a large series of anode radii and potentials for a $1 \mathrm{~cm}$ square silicon chip in plasma at $350 \mathrm{~km}$. The result of these calculations is a map of the available charge-tomass ratio over the anode's design space. Figure 5.11 presents these results as contours of charge-to-mass in $\mathrm{C} / \mathrm{kg}$ are given over a logarithmic range of sphere radii and potentials.

An important output of the solution flow-diagram is the required solar cell array area, dictated by the required power and the solar cell array's efficiency. The shaded regions in Figure 5.11 correspond to anode designs that require more surface area than is available on one side of the silicon chip. This requirement restricts the available design space to a combination of small radii and low potentials.

Reviewing the unshaded regions in Figure 5.11, charge-to-mass ratios as high as 7 $\mu \mathrm{C} / \mathrm{kg}$ can be achieved. After considering charge-to-mass ratio, available surface area for spacecraft subsystems, and simplicity of use; we select a radius corresponding to the corner-distance of the chip $(\sqrt{2} \mathrm{~cm})$ and a potential of $200 \mathrm{~V}$. These choices enable the chip to be placed within the sphere and attached at the corners, ensuring a constant orientation. With these parameters, the OML current model yields a chargeto-mass ratio of $2.92 \mu \mathrm{C} / \mathrm{kg}$ and the ram current model yields $2.55 \mu \mathrm{C} / \mathrm{kg}$. For the sake of conservatism, we adopt $2.5 \mu \mathrm{C} / \mathrm{kg}$ in further estimates. 


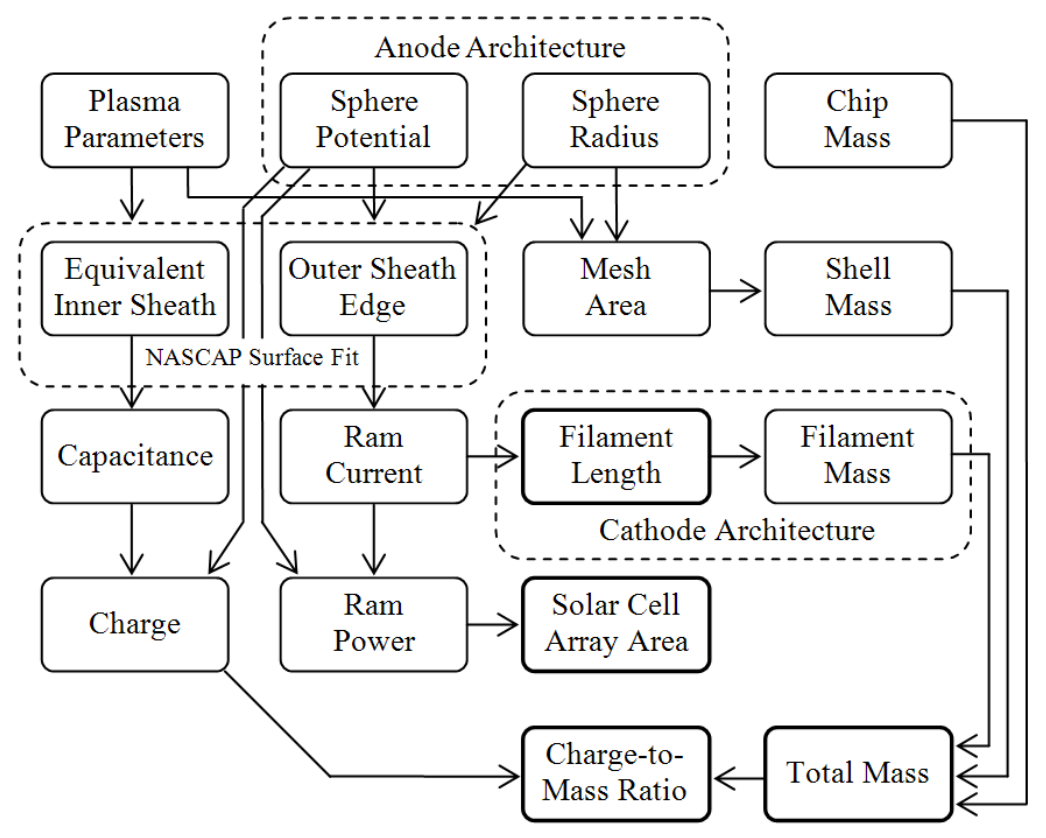

Figure 5.9. Solution flow-diagram for a differentially charged architecture with sheath currents associated with ram charging.

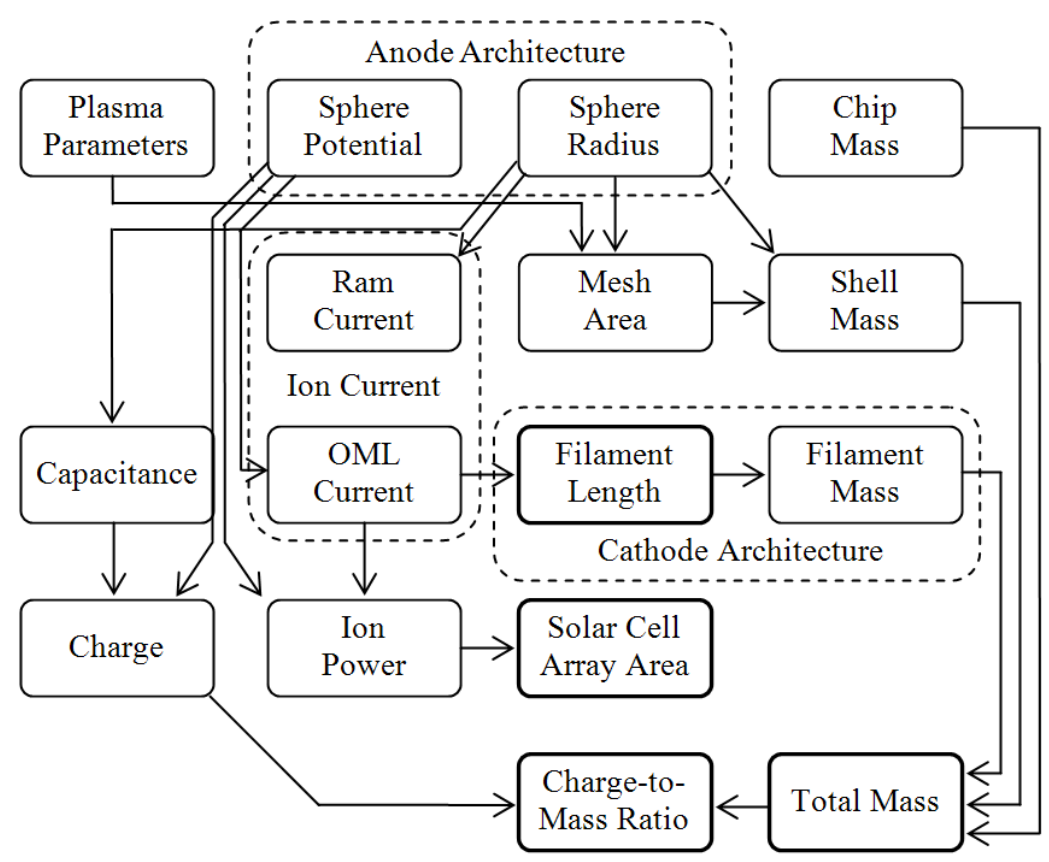

Figure 5.10. Solution flow-diagram for a differentially charged architecture with sheath currents associated with OML charging. 

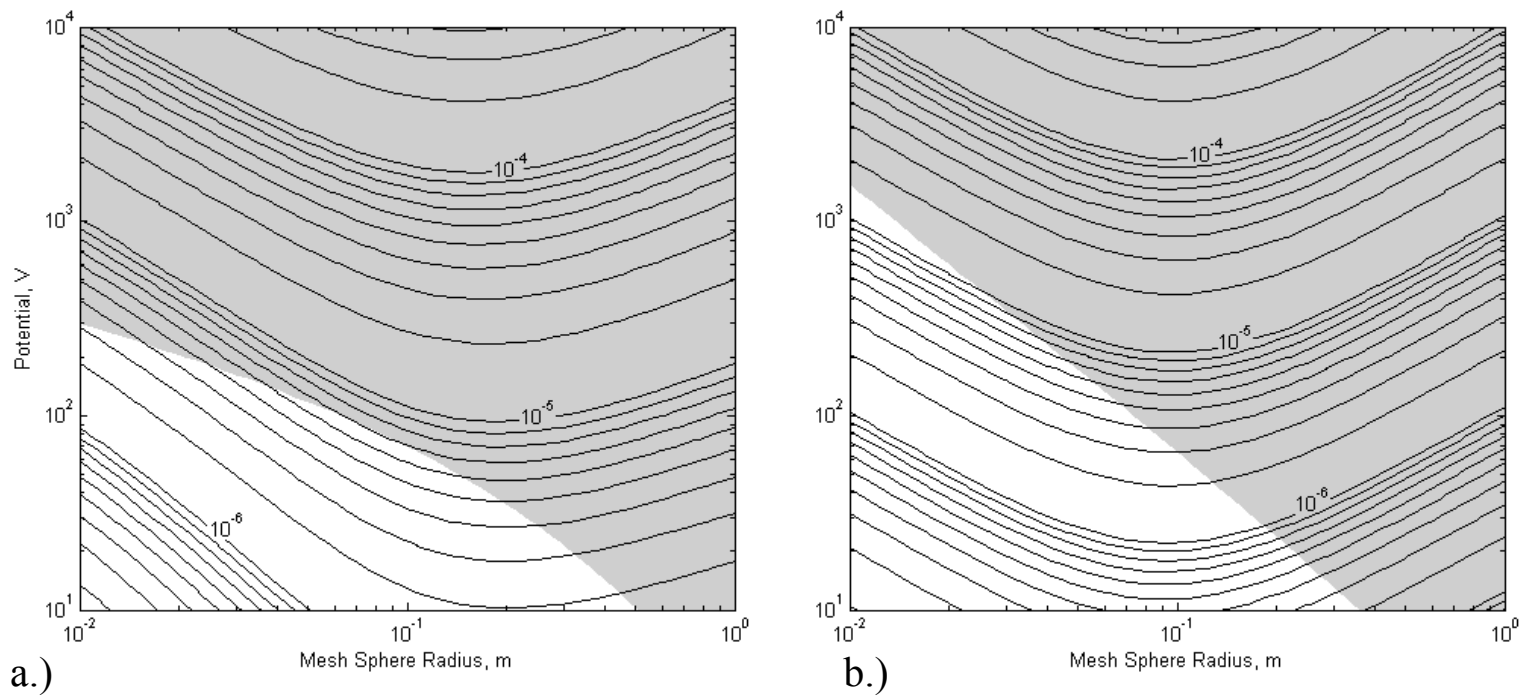

Figure 5.11. Contours of charge-to-mass in $\mathrm{C} / \mathrm{kg}$ for mesh sphere anode over ranges of radii and potential for a.) a ram charging dominated model and b.) an OML charging model. The shaded region indicates solutions requiring more solar-cell area than is available on one side of the chip.

\subsubsection{Communications}

Inspired by Sputnik, the communication system is intended to be a periodic beacon. The frequency of each pulse communicates the spacecraft's temperature. This section reviews relevant topics. Research in each is currently being pursued.

To ensure that the signal can be tracked from a ground station, it must be powerful enough to overcome atmospheric attenuation and other noise sources. The communication link's carrier-to-noise-ratio $C / N$ is a useful measure of goodness. This ratio is influenced by the signal's frequency, the orbit's altitude, the transmitter's losses and power, atmospheric conditions, and antenna efficiency. We can accomplish only so much on this chip. A simple way to improve the downlink is to select a highgain ground station. We propose to use one of the Deep Space Exploration Society's two 60 foot diameter parabolic dishes located in a radio-quiet region of Colorado. 
With an antenna gain of $43 \mathrm{~dB}$, this dish represents an extremely sensitive publicly accessible receiver.

A beacon pulse signal is well suited for our mission. A simple RC charging circuit can produce periodic bursts of power via transistor switching as illustrated in Figure 5.12. Solar power charges the capacitor until the transistor is opened, releasing the stored energy. This energy is sent through an oscillator and is emitted as RF energy via two antennas. This sequence results in a pulsed oscillating signal as depicted in

Figure 5.13.

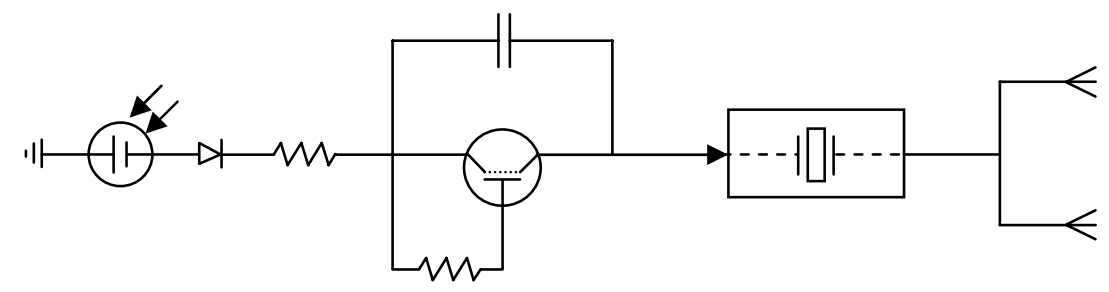

Figure 5.12. Conceptual circuit diagram to generate periodic RF pulses from solar flux.

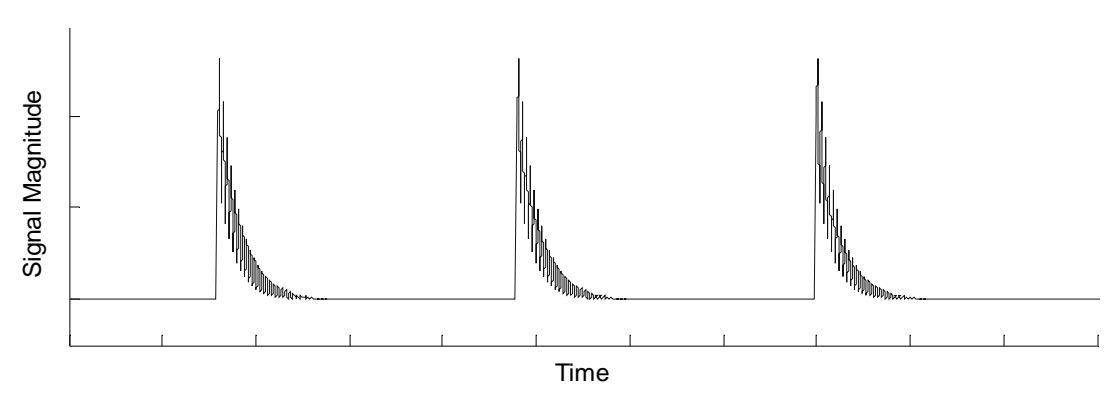

Figure 5.13. Conceptual RF pulsed output signal.

Thin-film deposition allows the fabrication of multi-layered parallel plate capacitors directly alongside other integrated circuit components. By alternatively 
depositing layers of metal and dielectric, charge can be stored efficiently over a given surface area. We use area-capacitance density as a metric in allocating the surface area of our wafer. Typical thin film capacitors, such as Metal-Insulator-Metal (MIM) achieve area-capacitance densities on the order of $10 \mathrm{nF} / \mathrm{cm}^{2}$. This performance can be improved by treating the surfaces between the plates and incorporating highdielectric materials, notably ceramics.

For the oscillator illustrated in Figure 5.12, the trade space has included an analog RLC circuit and a crystal oscillator. Though RLC circuit production is trivial at macroscopic scales, inductors are not well suited for microfabrication techniques. Research suggests that inductors on the order of $0.1 \mathrm{H} / \mathrm{mm}^{2}$ can be reliably produced, though the process in challenging. A second order RLC system resonates with a natural frequency of

$$
\omega_{R L C}=\frac{1}{\sqrt{L_{t c} C_{r c}}}
$$

where $C$ is capacitance in Farads and $L$ is inductance in Henries. At the millimeter scale, this range of capacitance $\left(1 \mu \mathrm{F} / \mathrm{mm}^{2}\right)$ and inductance $\left(0.1 \mu \mathrm{H} / \mathrm{mm}^{2}\right)$ yields a natural frequency on the order of $100 \mathrm{kHz}$. Alternatively, an RLC circuit can be reproduced using a crystal piezoelectric oscillator. When subjected to an electric field, a piezoelectric crystal deforms. As the field is removed, it mechanically returns to its natural state, a process that in turn creates an opposing electric field. This process repeats, resulting in an oscillating electric signal that can be modeled as the RLC circuit in Figure 5.14.

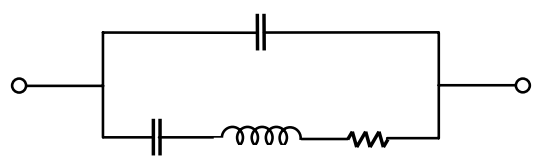

Figure 5.14. Piezoelectric crystal equivalent-analog circuit. 
The carrier frequency of the oscillator is another key parameter. Beyond dictating the necessary signal power and oscillator design, the frequency must conform to international law. The Federal Communications Commission (FCC) restricts transmissions to specific frequency ranges and power levels [16]. The influence of the frequency choice on signal attenuation is shown in Figure 5.15. It is difficult to imagine that our SOC spacecraft will have sufficient power to exceed the FCC limits at any frequency.

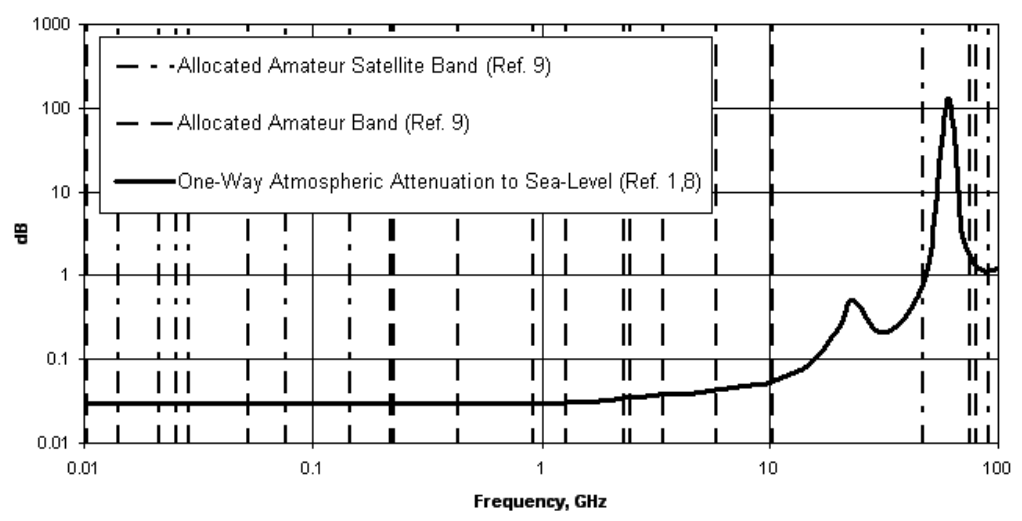

Figure 5.15. FCC allocated amateur bands superimposed over a model for one-way atmospheric attenuation to sea-level $\left(7.5 \mathrm{~g} / \mathrm{m}^{3}\right.$ water vapor).

Finally, it remains to discuss the antenna, a well researched topic in the microfabrication industry. Since attitude control is limited, directionality cannot be a design driver. If printed directly on the chip, only half of the energy will be radiated outward. Alternatively, one or more antennas could be mechanically attached to the circuit in the post-production and packaging fabrication steps. Though this approach would increase the antenna efficiency and enable a greater range of frequencies, it conflicts with our mass- and cost-saving goals that inspire a completely integrated spacecraft. 
Two antenna styles seem most practical, having been fabricated and demonstrated at our size scales: a linear dipole antenna and a loop dipole antenna. Dipole antennas can be designed for a resonant frequency by sizing the length at full, half, or quarter wavelengths. Using a quarter-wave dipole, a centimeter length limit constrains the minimum antenna resonant frequency to approximately $7.5 \mathrm{GHz}$. The second option, a loop antenna, offers increased gain over a dipole but also has equally restrictive frequency limits. For example, a $1 \mathrm{~cm}$ x $1 \mathrm{~cm}$ square loop yields a minimum resonance of approximately $8 \mathrm{GHz}$. Based on frequency allocation, ground-station capabilities, and atmospheric attenuation; these minimum frequencies may dictate limit the transmitter's design.

\subsubsection{Attitude Determination and Control}

Our proposed method of LAO via differentially charged bodies offers a passive method of attitude control. Figure 5.16 illustrates a sphere anode and filament cathode with center of mass moving at angular velocity $\omega_{+}$. The two oppositely charged geometries experience Lorentz forces in opposite radial directions concentrated at their respective centers of charge. If the spacecraft is perturbed from a radial alignment by angle $\alpha$, these forces apply a restoring torque. Thus the system behaves as a simple harmonic oscillator with an equilibrium at $\alpha=0$. This finding implies that the charged spacecraft's attitude is passively controlled by the same mechanism altering its orbit. Thus our spacecraft achieves a stable orbit-radial attitude with no additional mass, power, or system complexity. Because the orbit is circular, the spacecraft's attitude is constant with respect to nadir, offering the further advantage of ensuring the inside face of the chip always points towards Earth. This alignment ensures consistent antenna coverage for telecommunications. 


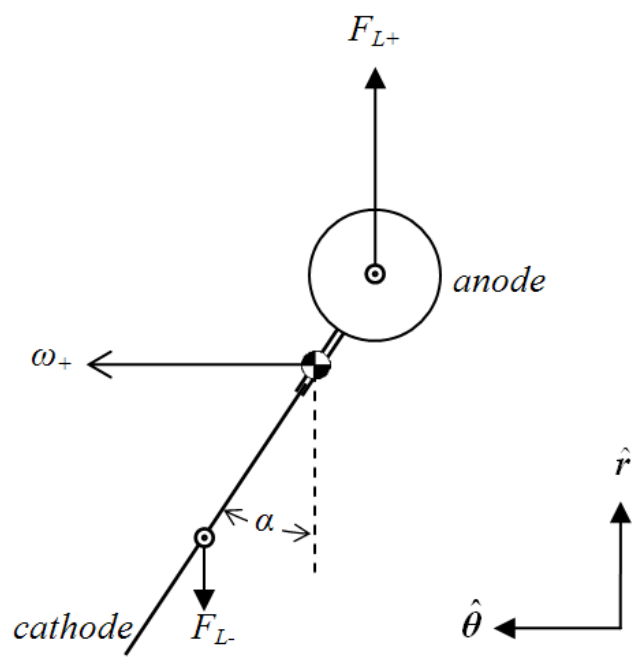

Figure 5.16. Illustration of differentially charged bodies subject to opposite Lorentz forces and free to rotate about the center of mass

For completeness we include discussion of another architecture we considered but chose not to pursue in this application: outgassing. Outgassing is the spontaneous evolution of a gas from a solid or liquid in the presence of a low-pressure environment. Spacecraft are built of low-outgassing materials in order to prevent the evolving gas cloud from corrupting sensors and payloads, from torquing the spacecraft, and from causing materials-related failures. As particles outgas from the surface, momentum is imparted to the spacecraft. By selectively applying an outgassing agent to the corners of the wafer, this momentum can be used to generate a torque on the spacecraft. This mechanism was observed as on the Microwave Anisotropy Probe spacecraft. There it caused undesirable change in angular momentum [17]. Once spinning, a SOC can use magnetic torque coils to precess the spinning attitude for active pointing. Such actuators scale well to the SOC and can be integrated easily as loop-shaped traces. 


\subsubsection{Structure}

The structure of the spacecraft consists of the single silicon chip. Though a gallium arsenide substrate offers improved radiation resistance and photovoltaic properties, it is not as readily used in standard fabrication processes or tools as silicon. In order to maximize use of the available surface area, we propose double-sided printing. Alternatively, a flip-chip style of production may be employed. In this process, two chips are manufactured such that their backsides can be mated and both chips face outward. This technique allows us to incorporate devices whose fabrication techniques aren't compatible by producing them separately and integrating them at the end of production. Of primary interest is the incorporation of solar cells on both sides of the chip to ensure that power is always available, regardless of attitude.

\subsubsection{Thermal Control}

In orbit, the SOC's low thermal mass will result in temperatures that range between $-130^{\circ} \mathrm{C}$ to $100^{\circ} \mathrm{C}$. This variation can occur within tens of seconds [18]. Thermal stresses associated with eclipses may fatigue the chip where dissimilar metals contact. These risks, along with possible remediative strategies such as microfabricated radiator fins, have yet to be evaluated. An active means of remediation might be achieved for the spinning SOC: tilting the attitude so that the face points toward or away from the sun or the earth to might be used to control temperature. 


\subsection{Candidate Design}

Our design features a $1 \mathrm{~cm}$ square, $500 \mu \mathrm{m}$ thick silicon chip surrounded by an aluminum mesh sphere and attached to an $8 \mathrm{~m}$ aluminum filament, as illustrated in Figure 5.17. Solar cells printed directly onto the chip supply $200 \mathrm{~V}$ at $48 \mu \mathrm{A}$ to the sphere and filament when oriented as much as $45^{\circ}$ from the sun. The sphere attains a negative potential $(-190 \mathrm{~V})$, and the filament's potential is weakly positive $(+10 \mathrm{~V})$. The two are separated by an insulated conductor that is longer than their combined outer sheath sizes to prevent plasma interactions. The system-level charge-to-mass ratio is $-2.5 \mu \mathrm{C} / \mathrm{kg}$ (calculated with the more conservative OML solution), sufficient for LAO demonstration when released into a circular orbit at $350 \mathrm{~km}$. The system passively orients itself along the Lorentz force's line-of-action. The total system mass amounts to less than $0.15 \mathrm{~g}$.

A separate solar array charges a capacitor designed to report the spacecraft's temperature as an RF pulse through a small antenna. Each side of the spacecraft is identical, to reduce solar array shadowing and transmission loss. Tables 5.4, and 5.5 give further design budgets and parameters.

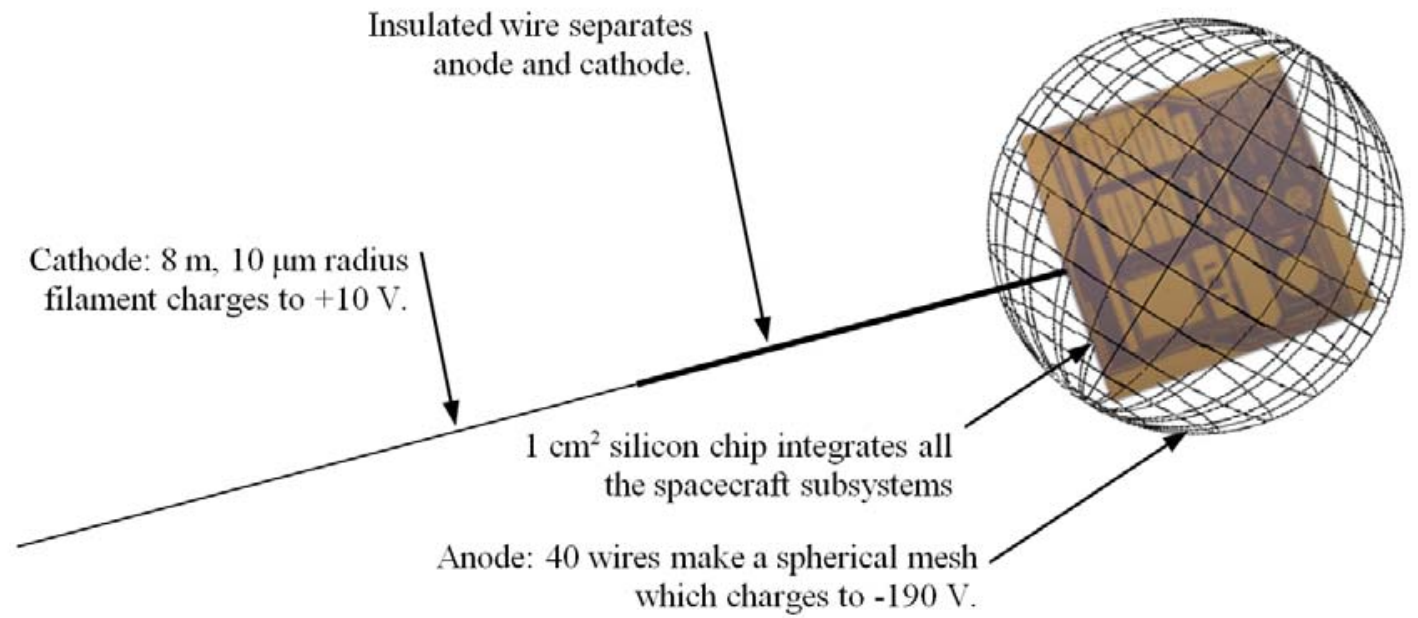

Figure 5.17. An illustration of our sample spacecraft architecture. 
Table 5.4. Minimum Mass Budget

\begin{tabular}{lcc}
\hline \hline Subsystem & Mass, $\mathrm{mg}$ & Surface Area per Side, $\mathrm{mm}^{2}$ \\
\hline Propulsion (Anode / Cathode) & 8 & 0, External \\
Telecommunications & 0, (Printed) & Remainder \\
Power & 0, (Printed) & 98 \\
Attitude Determination and Control & 0, Passive & 0, Passive \\
Structure & 115 & - \\
\hline Total & 123 & 157 \\
\hline \hline
\end{tabular}

Table 5.5. Selected Power and Propulsion Parameters

\begin{tabular}{lc}
\hline \hline Parameter & Selection / Result \\
\hline Orbit & Circular \\
Altitude, km & 350 \\
Quasi-Neutral Number Density, ${ }^{-3}$ & $1 \mathrm{E} 12$ \\
Quasi-Neutral Temperature, eV & 0.1 \\
Charging Method & Differential Charging \\
Potential Difference, V & 200 \\
Charge Storage & Mesh Sphere / Filament \\
Material & Aluminum \\
Filament Radius, m & $1 \mathrm{E}-5$ \\
Length Density, kg/m ${ }^{2}$ & $8.48 \mathrm{E}-7$ \\
Mesh Sphere Anode & \\
Radius, cm & $\sqrt{2}$ \\
Number of Filaments in Mesh & 40 \\
Filament Separation at diameter & Debye length \\
Total Length of Mesh, $\mathrm{m}$ & 1.8 \\
Total Mass, mg & 1.5 \\
Outer Sheath Edge, $\mathrm{m}$ & 0.14 \\
Equivalent Radius Sheath Size, $\mathrm{m}$ & 0.070 \\
Filament Cathode & \\
Length of Filament, m & 8.0 \\
Mass, mg & 6.8 \\
Outer Sheath Edge, $\mathrm{m}$ & $<0.1$ \\
Insulated Conductor Length, $\mathrm{m}$ & 0.25 \\
Solar Cell Array & \\
Specific Power & $100 \mathrm{~W} / \mathrm{kg}$ \\
Efficiency & $10 \%$ \\
Estimated Floating Potential, V & $3.59 \mathrm{E}-10$ \\
Net Charge, C & 48.5 \\
Ram Current, $\mu$ A & 2.5 \\
Estimated Charge to Mass Ratio, $\mu \mathrm{C} / \mathrm{kg}$ & 17.8 \\
Maximum Along-Track Separation, m/day & \\
\hline \hline & \\
& \\
\hline
\end{tabular}




\subsection{Conclusions}

We have motivated research into micro-scale design and fabrication of spacecraft components based on orbital dynamics, plasma effects, and propellantless technology. The result of these efforts is a candidate architecture for a fully integrated "Spacecraft on a Chip" capable of passively altering its orbit via the Lorentz force. Based on this work, we conclude that the concept is indeed viable, and the project is worth pursuing both for the sake of spacecraft integration and Lorentz Orbit Augmentation. Our current and future work focuses on addressing the telecommunications subsystem design, devising a robust procedure for estimating the spacecraft's orbit, and developing microfabrication process-diagrams. Ultimately, we intend to produce and launch the first Lorentz-propelled millimeter-scale spacecraft. 


\section{REFERENCES}

[1] Peck, M. A., Prospects and Challenges of Lorentz Augmented Orbits," Submitted for Publication. AIAA Journal of Spacecraft and Rockets. 2006.

[2] Streetman, B. and Peck, M., "Synchronous Orbits and Disturbance Rejection Using the Geomagnetic Lorentz Force" Proceedings of the AIAA Guidance, Navigation, and Control Conference, August 2006.

[3] Hillard G. B. and Ferguson, D. C., "Low Earth Orbit Spacecraft Charging Design Guidelines," NASA/TP-2003-212287, February 2003.

[4] Hoyt, R. P., and Minor, B. M., "Remediation of Radiation Belts Using Electrostatic Tether Structures," Tethers Unlimited Inc., 2004.

[5] Whipple. E. C., "Potentials of Surfaces in Space," Reports on Progress in Physics, Vol. 44, 1981, pp. 1217

[6] Blackwell D. D., Walker, D. N., Messer, S. J., and Amatucci, W.E., "Characteristics of the Plasma Impedance Probe with Constant Bias," Physics of Plasmas, Vol 12. 2005.

[7] Laframboise, J. G., Ph.D. thesis, University of Toronto, Institute for Aerospace Studies, Report No. 100, 1966.

[8] Parker, L.W., "Plasmasheath-Photosheath Theory for Large High-Voltage Space Structures," AIAA, 1980. p. 507-509.

[9] Choinière, E., "Theory and Experimental Evaluation of a Consistent Steady-State Kinetic Model for 2-D Conductive Structures in Ionospheric Plasmas with Applications to Bare Electrodynamic Tethers in Space," Ph.D. thesis, University of Michigan, 2004.

[10] Kasha, M.A., "The Ionosphere and Its Interaction with Satellites," Gordon and Breach, New York, 1969.

[11] Geuther, J.A. and Danon, Y., "Electron and Positive Ion Acceleration with Pyroelectric Crystals," Journal of Applied Physics, Vol. 97, 2005. 
[12] Rossi, C., Larangot, B., Pham, P.Q., Briand, D., de Roij, N.F., Puig-Vidal, M., and Samitier, J., "Solid Propellant Microthrusters on Silicon: Design, Modeling, Fabrication, and Testing," Journal of Microelectromechanical Systems, Vol. 15, No. 6, 2006.

[13] Talley, C., Clayton, W., Gierow, J., McGee., J and Moore, J., "Advanced Membrane Materials for Improved Solar Sail Capabilities," 43rd AIAA/ASME/ASCE/AHS/ASC Structures, Structural Dynamics, and Materials Conference: 3rd AIAA Gossamer Spacecraft Forum, 2002.

[14] Cosmo, M. and Lorenzini, E. Editors, "Tethers in Space Handbook," 3rd ed, 1997.

[15] Kressmann, R., Sessler, G. M., and Günther, P., "Space-charge Electets," IEEE Transactions on Dielectrics and Electrical Insulation, Vol. 3, No. 5, 1996.

[16] U.S. Department of Commerce, National Telecommunications and Information Administration, Office of Spectrum Management, "United States Frequency Allocations - The Radio Spectrum," October 2003.

[17] Starin, S. R. et al, “An Anomalous Force on the MAP Spacecraft,” 2002 AIAA Symposium, Monterey, CA.

[18] Gilmore, D. G., Hardt, B. E., Prager, R. C., Grob E. W. and Ousley, W., "Thermal Systems," Spacecraft Mission Analysis and Design, 3rd ed., Microcosm Press and Kluwer Academic Publishers. 2005. 


\section{CHAPTER 6 \\ LORENTZ AUGMENTED JOVIAN ORBIT INSERTION*}

\subsection{Abstract}

The Lorentz force acting on a statically charged body moving with respect to a rotating magnetic field is evaluated as a means of capture into a Jovian orbit. The study offers insight into the classes of captures available as a function of the spacecraft's charge-to-mass ratio and approach conditions. A range of these parameters is simulated using low-order magnetosphere and gravity models and a bang-off controller. The results suggest that charge-to-mass ratios on the order of 0.1 $\mathrm{C} / \mathrm{kg}$ are required to capture a spacecraft with a hyperbolic, equatorial approach to an orbit similar to that of Jupiter's moon Europa. The Lorentz Augmented Orbits (LAO) architecture is related to a similar technology, electrodynamic tethers (EDT). Using a high-order magnetic field and gravity model, we recreate a sample EDT Jovian capture mission using LAO with a charge-to-mass ratio of $0.0975 \mathrm{C} / \mathrm{kg}$ over 2.5 years. We conclude that LAO maneuvers are capable of reducing a spacecraft's energy and eccentricity into a bounded, circular Jovian orbit for typical mission requirements. A simple feasibility study suggests that LAO spacecraft designs capable of LAO Jovian orbit insertion are challenging, but likely possible.

\footnotetext{
* Reproduced with permission from Journal of Guidance, Control, and Dynamics. Originally published in Vol. 32, No.2, 2009, pp. 418-423.
} 


\subsection{Introduction}

Dust detectors on Galileo observed a diffuse ring of exogenic material with primarily retrograde orbits around Jupiter [1]. Subsequent analysis demonstrated that interplanetary and interstellar dust is captured into these stable, bound orbits via timevarying Lorentz interactions with Jupiter's magnetic field [2]. This research inspires us to ask whether we can exploit this perturbing effect for orbit control by purposely generating and modulating electric charge on a spacecraft.

The purposes of this study are to gain insight into the orbital mechanics of chargemodulated bodies at Jupiter and to evaluate the feasibility of utilizing the Lorentz force for spacecraft capture and orbit circularization. Jupiter generates both the strongest and fastest-rotating magnetic field in the solar system. With a 9.93 hour spin period and a dipole magnetic moment roughly 20,000 times that of Earth [3], Jupiter is the most charge-efficient body where such Lorentz effects can be demonstrated. Previous work has evaluated Lorentz Augmented Orbits (LAO) [4] as a means of gaining energy at Jupiter during fly-by maneuvers [5]. This study addresses the inverse problem: using LAO to reduce energy within given time and space constraints. The term capture describes a decrease in a satellite's kinetic energy sufficient to place it in a stable orbit within Jupiter's Hill sphere. Spacecraft missions to outer planets typically rely on a combination of gravity-assist maneuvers and chemical propulsion for capture. The Galileo mission, for example, used a fly-by of Io and a 49 minute thruster burn. Reliance on flybys introduces complexity in mission planning and restrictions on launch windows. Thruster use adds to the spacecraft total mass, in Galileo’s case, $371 \mathrm{~kg}$ of fuel. LAO requires only that a spacecraft establish a biased electrostatic charge and therefore does not use fuel for orbital maneuvers. This study evaluates whether LAO can produce capture maneuvers comparable to those that 
traditional propulsion can achieve. The results include a range of parameters associated with the satellite's charge, lifetime, and approach conditions and compare the performance to a related propellantless propulsion technology, electrodynamic tethers.

\subsection{Reduced-Model Equations of Motion}

We treat the spacecraft-Jupiter system as a restricted two-body problem. Spherical coordinates provide a set of basis vectors that conveniently describe the system, $(\hat{\boldsymbol{r}}, \hat{\boldsymbol{\varphi}}, \hat{\boldsymbol{\theta}})$ where $\hat{\boldsymbol{r}}$ is the unit vector in the radial direction, $\hat{\boldsymbol{\varphi}}$ is the unit vector in the polar angle direction, and $\hat{\boldsymbol{\theta}}$ is the unit vector in the azimuthal direction. The Lorentz force is given by

$$
F_{L}=q v_{r} \times B
$$

where $\boldsymbol{F}_{\boldsymbol{L}}$ is the Lorentz force experienced by the spacecraft with charge $q$ and velocity $\boldsymbol{v}_{\boldsymbol{r}}$ relative to Jupiter's magnetic field $\boldsymbol{B}$. This relative velocity is

$$
v_{r}=\dot{r}-\omega_{B} \times r,
$$

where $\dot{r}$ is the vector time derivative in a Newtonian frame of $\boldsymbol{r}$, the position of the satellite, and $\omega_{\boldsymbol{B}}$ is the angular velocity vector of Jupiter. The relative velocity is maximized in retrograde equatorial orbits. Dividing by the spacecrafts mass yields acceleration $\boldsymbol{a}_{\boldsymbol{L}}$ and identifies a critical parameter: $q / m$, the satellite's charge-to-mass ratio

$$
\boldsymbol{a}_{\boldsymbol{L}}=\frac{q}{m}\left(\dot{\boldsymbol{r}}-\boldsymbol{\omega}_{\boldsymbol{B}} \times \boldsymbol{r}\right) \times \boldsymbol{B}
$$


Eq. 3 makes clear that the direction of the Lorentz force is determined by the spacecraft's orbit and the central body's magnetic field and rate of rotation. If only a single polarity of charge is possible, the spacecraft can actuate only this scalar magnitude, varying the magnitude but not the direction of the resulting Lorentz force. If both polarities are possible, the direction is still constrained instantaneously to a one-dimensional manifold.

Jupiter's magnetosphere is a subtle, time-varying structure featuring a dominant tilted-dipole component, an equatorial current disk, and a pronounced magnetotail [6]. Here, in the interest of clarity, we model this structure simply as a dipole aligned with Jupiter's spin axis. Simulations show that this approximation captures the essential features of the phenomenon [7]. Given a magnetic induction value $B_{0}$ at a reference altitude $r_{0}$, an axis-aligned dipole magnetic field is

$$
\boldsymbol{B}=-B_{0}\left(\frac{r_{0}}{r}\right)^{3}(2 \cos \phi \hat{\boldsymbol{r}}+\sin \phi \hat{\boldsymbol{\varphi}})
$$

Where $\phi$ and $r$ constitute two of three spherical coordinates. The Lorentz force in such a basis is then:

$$
\boldsymbol{F}_{\boldsymbol{L}}=q B_{0}\left(\frac{r_{0}}{r}\right)^{3}\left(r \sin ^{2} \phi\left(\dot{\theta}-\omega_{B}\right) \hat{\boldsymbol{r}}+r \sin (2 \phi)\left(\dot{\theta}-\omega_{B}\right) \hat{\boldsymbol{\varphi}}+(2 r \dot{\phi} \cos \phi-\dot{r} \sin \phi) \hat{\boldsymbol{\theta}}\right) .
$$

For a restricted two-body, drag-free, system confined to equatorial orbits, the equations of motion reduce to

$$
\begin{gathered}
\ddot{r}=r \dot{\theta}^{2}-\frac{\mu}{r^{2}}+\frac{q}{m} \frac{B_{0} r_{0}^{3}}{r^{2}}\left(\dot{\theta}-\omega_{B}\right), \\
r \ddot{\theta}=2 \dot{r} \dot{\theta}-\frac{q}{m} \frac{B_{0} r_{0}^{3}}{r^{3}} \dot{r} .
\end{gathered}
$$


Higher-order gravitational and magnetic terms, as well as non-zero inclinations, are considered in Section IV.

Figure 6.1 shows the directions of these vectors at three positions $\left(\boldsymbol{r}_{1}, \boldsymbol{r}_{2}, \boldsymbol{r}_{3}\right)$ within an arbitrary retrograde elliptical, equatorial orbit at Jupiter. At the magnetic equator, the magnetic field lines point southerly and are thus directed into the page. For each of the three positions shown, the Lorentz force acts along $\boldsymbol{v}_{\boldsymbol{r}} \times \boldsymbol{B}$ and is shown with an arbitrary magnitude.

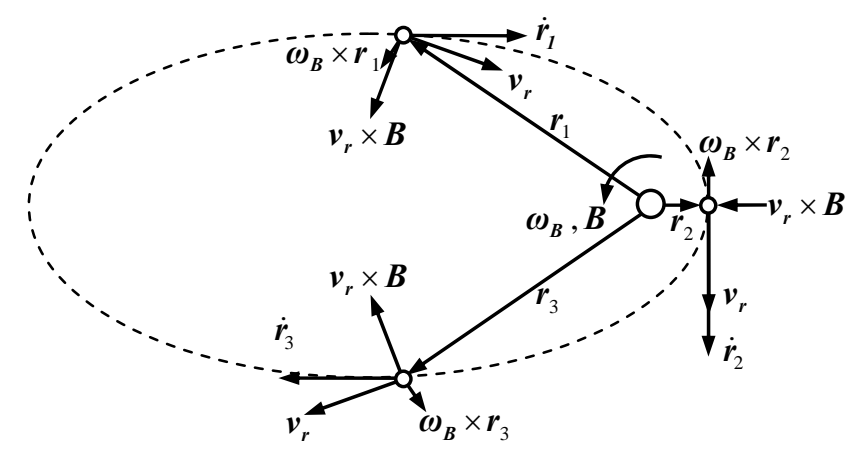

Figure 6.1. Vector depiction of the Lorentz force at various locations in an elliptic retrograde magnetic-equatorial orbit.

The Lorentz force is nonzero in an equatorial orbit, except where $\dot{\boldsymbol{r}}=\boldsymbol{\omega}_{B} \times \boldsymbol{r}$, as in GEO. The change in specific energy $E$ in an inertial reference frame is found by projecting the Lorentz force onto the inertial velocity vector, $q\left(\boldsymbol{v}_{\boldsymbol{r}} \times \boldsymbol{B}\right) \cdot \dot{\boldsymbol{r}}$. In an equatorial orbit, this term is related to the radial component of the velocity [7]

$$
\dot{E}=\frac{q}{m} \omega_{B} \frac{B_{0}}{r^{2}}(\dot{\boldsymbol{r}} \cdot \hat{\boldsymbol{r}}) .
$$

This quantity is nonzero except in a circular orbit or at the apsides, where $\left(\boldsymbol{v}_{\boldsymbol{r}} \times \boldsymbol{B}\right) \perp \dot{\boldsymbol{r}}$, indicating that the Lorentz force can change the energy of an orbit. However, the force's symmetry across the line of apsides suggests that the cumulative effect over an 
orbit is zero. To achieve a secular energy gain or loss, the magnitude of the charge must exhibit asymmetry about this line. This issue of symmetry also suggests that a given energy maneuver can be accomplished with either polarity of charge. For example, a spacecraft charged positive while approaching perijove sees a reduction in its orbital energy similar to that of a spacecraft charged negative while leaving perijove. In prograde orbits however, one must consider the $\left(\dot{\theta}-\omega_{B}\right)$ terms in Eqs. 5 and 6. The sign of these terms can vary through an orbit, for example as a spacecraft in a near-circular orbit passes the geostationary orbital radius.

We use MATLAB's fourth-order Runge-Kutta integrator to solve the set of four first-order, coupled equations of motion derived from Eqs. 6 and 7 with a range of initial conditions. The algorithm uses an adaptive time step such that the absolute integration error in the radius vector does not exceed $10^{-5} \mathrm{~m}$. The simulations are confined to regions within Jupiter's Hill sphere radius $R_{H}$. Table 1 gives the constants that are used in all of these simulations.

Table 6.1. Jovian constants used in all simulations

\begin{tabular}{cc}
\hline$\mu$ & $1.2670 \times 10^{17} \mathrm{~m}^{3} / \mathrm{s}^{2}$ \\
$r_{0}$ & $\mathrm{R}_{\mathrm{J}}, 7.1492 \times 10^{7} \mathrm{~m}$ \\
$\omega_{J}$ & $1.7735 \times 10^{-4} \mathrm{rad} / \mathrm{s}$ \\
$B_{0}$ & $-4.26 \times 10^{-4} \mathrm{~T}$ \\
$R_{H}$ & $740 \mathrm{R}_{\mathrm{J}}$ \\
\hline
\end{tabular}

This LAO spacecraft is assumed to be capable of maintaining a constant charge in spite of environmental discharging effects. We first consider an initially parabolic approach. This choice reduces the orbit to a single parameter, the perijove radius and offers useful insights into the orbit-insertion problem. We select a perijove radius of 4 
$R_{J}$ and initialize the simulation at a distance of $50 R_{J}$. The conservatism in these approach conditions acknowledges the limitations of the model, including simplifications to the magnetic field and the absence of an atmospheric-drag model.

\subsection{Constant Charge}

If the charge $q$ is a positive constant, the satellite sheds energy and momentum as it approaches perijove, where the radial velocity vector changes direction. Past perijove, the satellite accelerates because the projection of the Lorentz force onto the velocity vector results in a positive moment about the barycenter.

The time histories for a range of six constant-charge approaches are shown in Figure 6.2. The results verify the theoretical result that a spacecraft cannot be captured if the charge is not modulated. When charged, the spacecraft exchanges orbital energy with the spin of Jupiter. As predicted, energy is removed while the spacecraft approaches perijove and subsequently is returned while the spacecraft approaches apojove. This trend is evident in Figure 6.3, which shows the spacecraft's total specific energy over time. Likewise, eccentricity has a net zero change over the course of an orbit, as shown in Figure 6.4. The energy transfer occurs most rapidly near perijove, where the spacecraft's relative velocity and the planet's magnetic-field magnitude are maximized. 


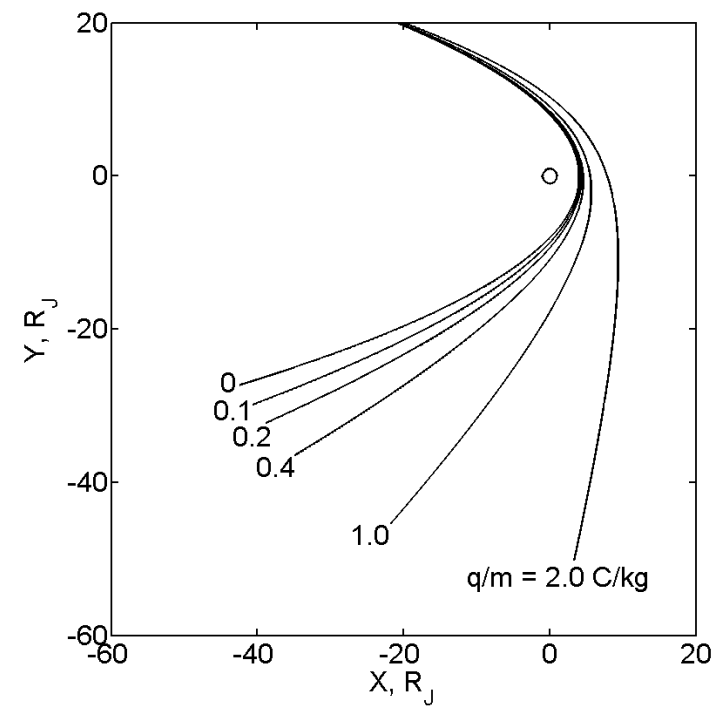

Figure 6.2. Orbit time-histories for constant charge-to-mass spacecraft with parabolic approaches.

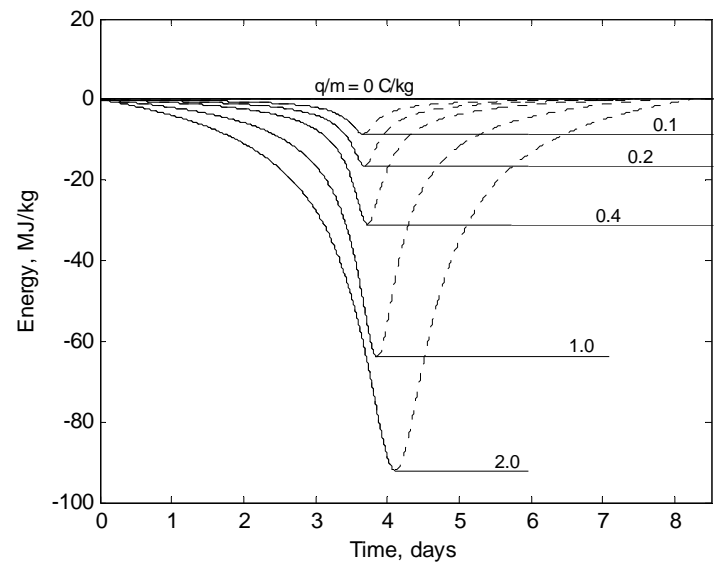

Figure 6.3. Energy time-histories for constant (dashed) and bang-bang actuated (solid) charge-to-mass spacecraft with parabolic approaches. 


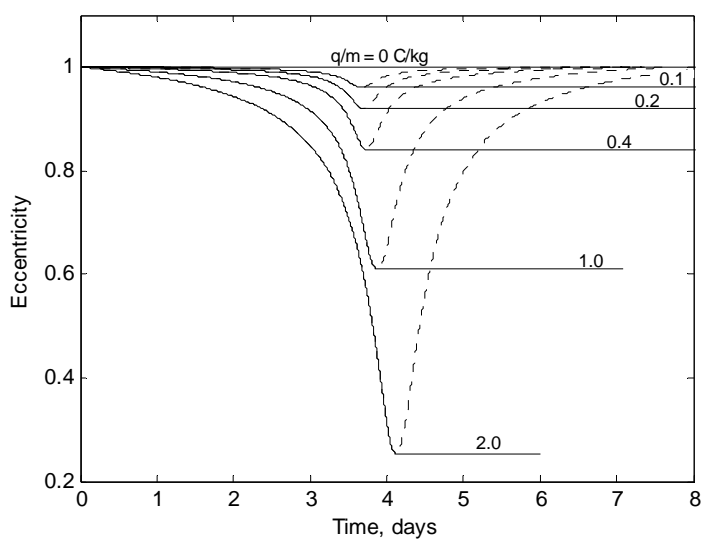

Figure 6.4. Eccentricity time-histories for constant (dashed) and bang-off actuated (solid) charge-to-mass spacecraft with parabolic approaches.

\subsection{Bang-Off Charge-Control}

The principle of Hamilton-Jacobi Bellman optimality inspires a bang-off control strategy to actuate the spacecraft's charge $[8,9]$. We neglect the finer points of disturbance cancellation and control of dynamics other than those of the two-body problem. The bang-off charge-control method uses the maximum control effort throughout the interval of operation, in this case, half of the orbit. That is, the spacecraft supplies its maximum charge over half of the orbit in order to produce the maximum effect over a given time. This control method can be expressed with a Heaviside step function $H$ that depends on true anomaly $f$, in this case over the half of the orbit approaching apoapsis,

$$
\frac{q}{m}(f)=\left(\frac{q}{m}\right)_{\text {max }} H(f-\pi) .
$$

This controller is optimal in terms of time, in that it achieves a desired orbital evolution in minimum time [10]. However, this controller is suboptimal in terms of power, in that it maintains a charge even when the Lorentz force is insignificant (for example, near apojove in a highly eccentric orbit). Figure 6.5 shows the orbit time- 
histories of six sample cases using this control approach. The corresponding energy and eccentricity time-histories are given in Figs. 6.3 and 6.4 respectively. The charge is applied while the radius is decreasing (approaching the perijove) and removed while the radius is increasing (approaching apojove).

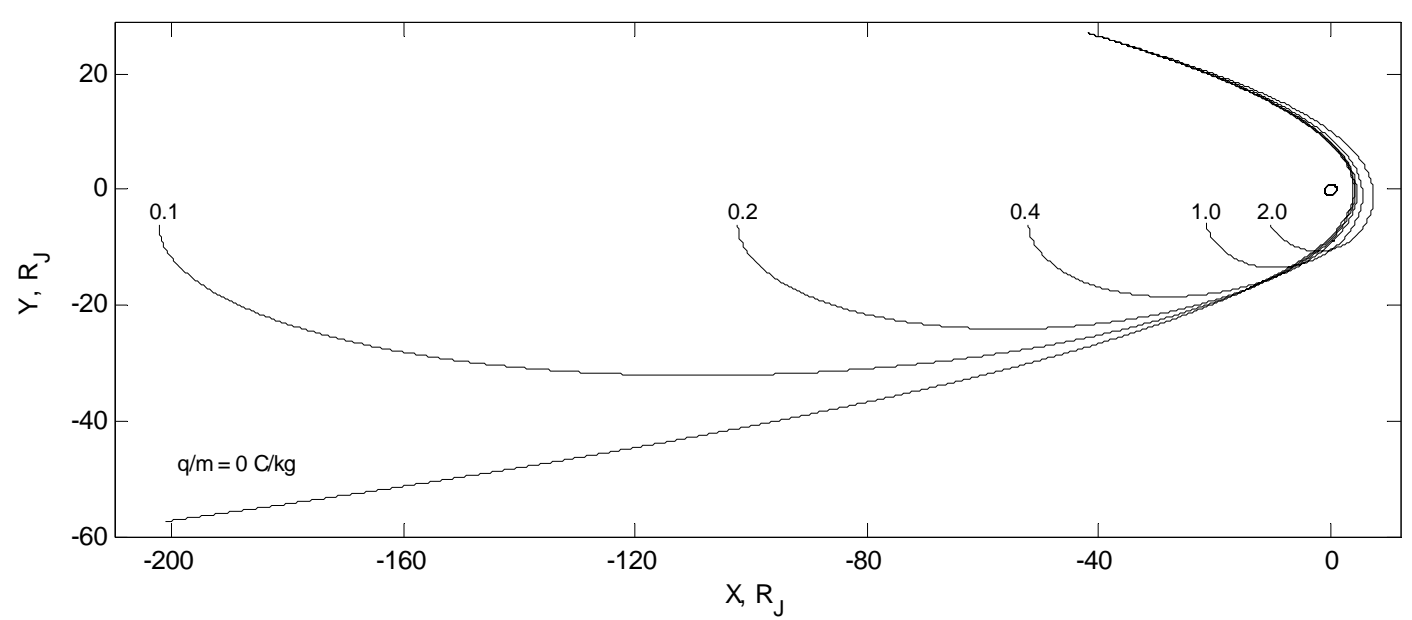

Figure 6.5. Orbit time-histories for spacecraft with bang-off actuated charge-to-mass ratios.

To implement this controller, we use true anomaly as an input and incorporate a 10 second continuous transition time [11]. This charge transition better models the physics of the discharge than a step function, improves the numerical integration speed, and helps ensure the stability of the closed-loop system which otherwise can exhibit high-frequency limit-cycle behaviors.

\subsection{Approach Space}

In an effort to better understand the capture opportunities and responses over a large space of approach conditions, we simulate a series of test cases for planar nonparabolic orbits with varying semimajor axis $a$ and eccentricity $e$. Using 1280 (a,e) 
pairs and four $q / m$ ratios, these simulations numerically explore the effectiveness of bang-off charge-control for capture maneuvers. Here, a "successful capture" corresponds to a final orbit of useful size and shape for missions of possible interest: semimajor axis less than $10 \mathrm{R}_{\mathrm{J}}$ and an eccentricity less than 0.10 . This near-circular orbit is close to that of Europa. Simulations are initialized at either apojove or $50 R_{J}$ and are run to a maximum time of five years.

The result of these simulations is a map of the capture performance for each $q / m$ ratio over the approach space, shown in Fig. 6.6 (a-d). The approach orbit's eccentricity is on the ordinate, and the approach orbit's semimajor axis is on the abscissa. The figure shows dashed lines of constant perijove to illustrate the importance of this parameter's effect on the charged spacecraft's behavior. The successfully captured cases are sorted by their time to capture. The unsuccessful cases are sorted by four observed behaviors: planetary impact, escape, not captured within the five-year limit, and circular orbits.

The cases resulting in escape are limited to the hyperbolic portion of the plots in which applying charge cannot reduce the energy and momentum during the spacecraft's first approach enough to bound its orbit within Jupiter's Hill sphere. An increased $q / m$ can prevent escape for a given hyperbolic approach. Likewise, increased charge reduces the number of orbits which impact the planet. Impact cases are those that carry the spacecraft closer than two $\mathrm{R}_{\mathrm{J}}$ from the barycenter. Increased charge can reduce eccentricity rapidly enough to maintain a sufficient perijove radius. The approach conditions and $q / m$ values that lead to neither an impact nor our definition of capture within the five-year limit appear in the elliptical portion of the low-charge plots.

Finally, there are many cases identified as circular orbits. These orbits are characterized by eccentricities less than 0.005 and semimajor axes greater than $10 \mathrm{R}_{\mathrm{J}}$. 
They represent undesired equilibria. As the eccentricity decreases, so too does the ability to reduce energy. In a circular orbit, the Lorentz force is purely radial and thus cannot do work on the orbit. In these cases, the spacecraft is trapped and cannot achieve capture within any time period. With a more subtle controller, it may be possible to reduce energy and angular momentum in such a way that this result is prevented. Therefore, these cases identified as circular orbits in the plots may represent prospective captures.

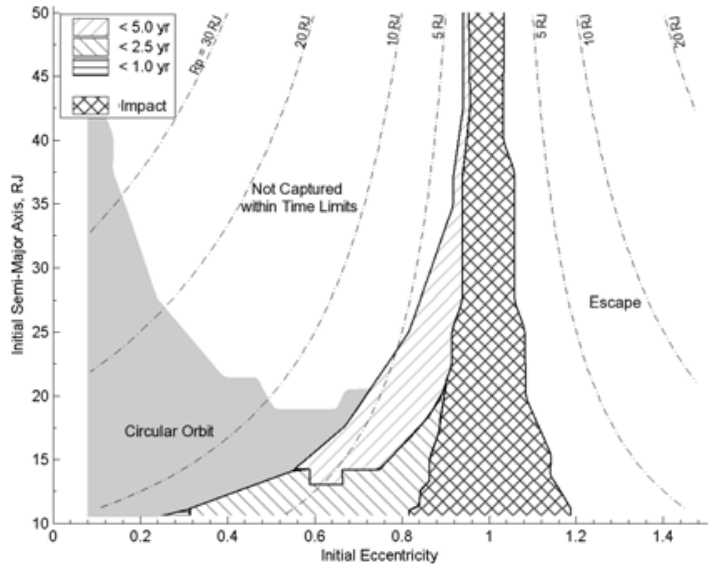

a.) $q / m=0.005$

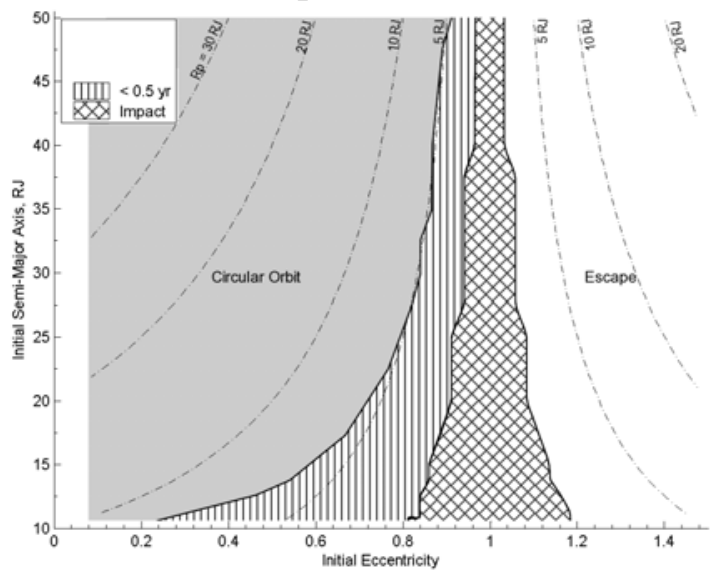

c.) $\mathrm{q} / \mathrm{m}=0.10$

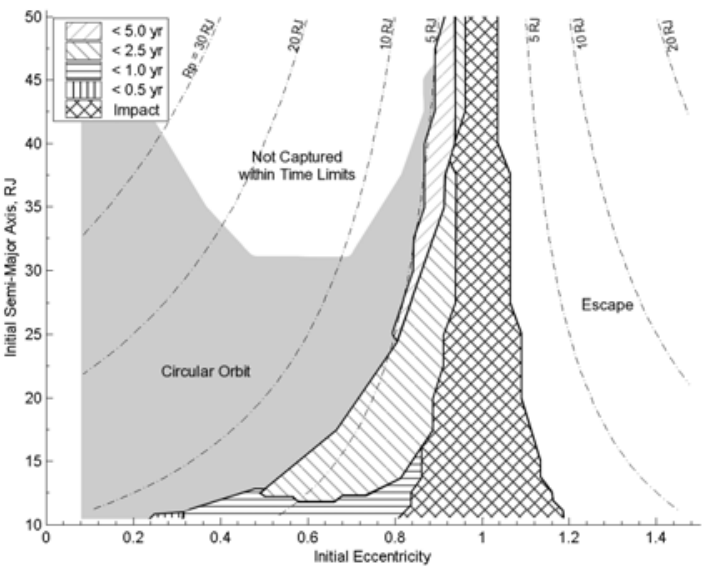

b.) $q / m=0.010$

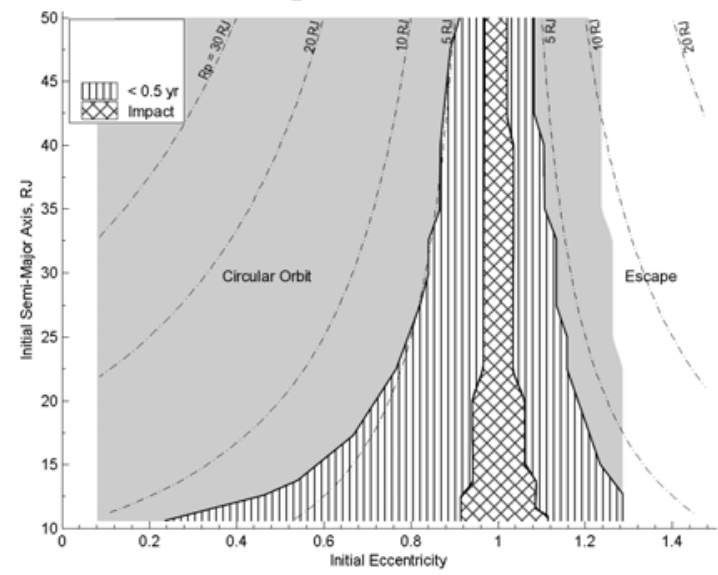

d.) $\mathrm{q} / \mathrm{m}=1.0$

Figure 6.6 (a-d). LAO captures for a range of initial conditions and charge to mass ratios. Contours of constant perijove shown with dashed lines. 


\subsection{Electrodynamic Tether Comparison}

A related technology, electrodynamic tethers (EDT), offers similar mission benefits. An electrodynamic tether is a conductor of length $l$ through which a current $\boldsymbol{I}$ is supplied. In the presence of a magnetic field, the system experiences a force due to Lenz’s law

$$
\boldsymbol{F}_{T}=l \boldsymbol{I} \times \boldsymbol{B} .
$$

Conversely, motion of a conductor in a magnetic field induces a potential difference within the EDT. When surrounded by plasma, the EDT and plasma act as a closed circuit, and the induced potential difference drives a current. The power driven along the tether can then be stored in a battery or dissipated through resistance. The EDT's orbital energy is effectively converted to electrical energy.

LAO transfers a spacecraft's orbital energy to and from the central body’s rotation. In the presence of plasma, the spacecraft must counter the currents associated with plasma discharging in order to maintain a biased charge. With respect to power, LAO maneuvers are most efficient in the absence of plasma, whereas EDT maneuvers prefer dense plasmas to maximize the induced current flow.

The force on an EDT is a function of the current $\boldsymbol{I}$, a vector valued quantity. An EDT can therefore alter both the direction and magnitude of its acceleration by controlling to the attitude of the tether. However, this added degree-of-freedom introduces complexity: stability and controllability of the resulting librations limit the usefulness of tethers in some applications [12].

Jupiter is an appealing candidate for EDT capture demonstrations for the same reasons we cite. Early studies illustrated their potential for both capture and thrust at the Jovian equator $[13,14]$. Gallagher, Johnson, Moore, and Bagenal first extensively 
evaluated the feasibility and limitations of an EDT Jovian orbit insertion using high order models of the magnetic field and plasma environment [15]. A further study in 2000 extended these results for rotating tethers [16]. We take these two studies as a basis for evaluating LAO. More recently, Sanmartín et al. formally described equatorial EDT phased capture maneuvers using more accurate plasma current models [17]. These results were then used to design and analyze a complete system architecture that accounts for power, thermal, and dynamic loading [18].

\subsection{High-Order Model}

We devise an LAO that reproduces the performance described in Refs [15] and [16] by restricting the system to a planar orbit about the magnetic equator. We infer the studies' initial and final conditions, as presented in Table 2. These conditions were initially inspired by two potential NASA missions, the Radio Science Observer and the Auroral Observer [15]. Each simulation starts at the outer edge of Jupiter's Hill sphere.

Table 6.2. Initial and Captured Orbits

\begin{tabular}{lll}
\hline \hline Elements & Approach & Final \\
\hline$a$ & $-37.725 \mathrm{R}_{\mathrm{J}}$ & $<86 \mathrm{R}_{\mathrm{J}}$ \\
$e$ & 1.027 & $<0.988$ \\
\hline
\end{tabular}

The model of section II is expanded to include higher-order effects. Like previous studies, we use a magnetic field model from Khurana [19]. This model consists of the Goddard Space Flight Center's $\mathrm{O}_{6}$ internal field model coupled with an Euler potential 
formulation external field model. We augment the gravitational field model to include dipole and quadrapole terms but neglect other bodies.

\subsection{Results}

Our LAO version of the EDT maneuver uses a bang-off control strategy, which we evaluate over a range of $q / m$. Figure 6.7 shows the simulated time-to-capture as a function of $q / m$. A minimum $q / m$ of $0.0975 \mathrm{C} / \mathrm{kg}$ is required to complete a capture within the bounds of Jupiter's Hill sphere. This capture requires 870 days for the initial approach and a single subsequent orbit to achieve the target capture. Increasing $q / m$ reduces the time required to complete the capture, but only to a limited extent. Above $q / m=0.128 \mathrm{C} / \mathrm{kg}$, the spacecraft is captured within the initial Jovian approach, corresponding to 76.5 days. Given additional time, LAO continues to circularize and shrink the orbit as seen in Figure 6.8 where the $0.128 \mathrm{C} / \mathrm{kg}$ spacecraft is simulated for a total of 211 days. Figures 6.9 and 6.10 show the resulting radius and eccentricity time histories. The final orbit is defined by a semimajor axis of $3.7 R_{J}$ and an eccentricity of 0.052 after 211 days. This performance is competitive with the EDT candidate capture which required times on the order of hundreds of days with an 11 $\mathrm{km}$ tether. 


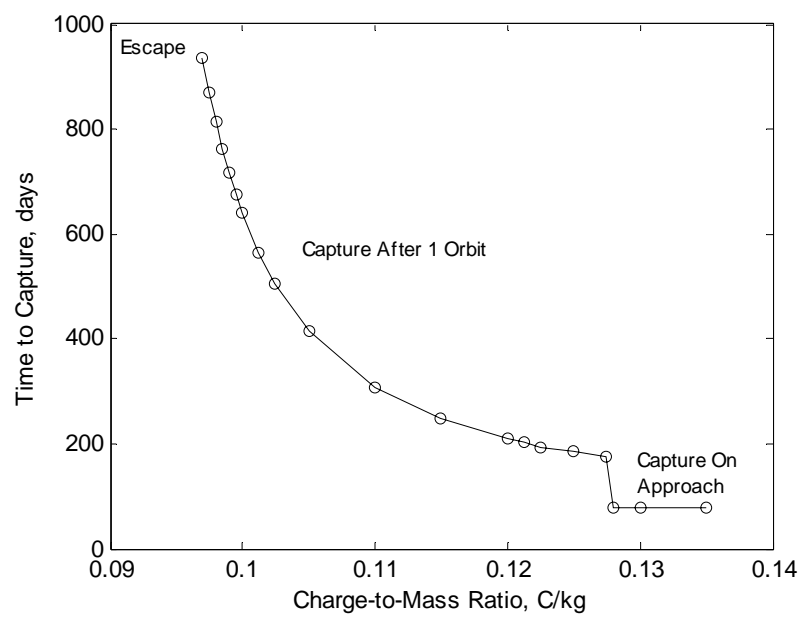

Figure 6.7. Time-to-capture as a function of charge-to-mass ratio for high-order model simulations.

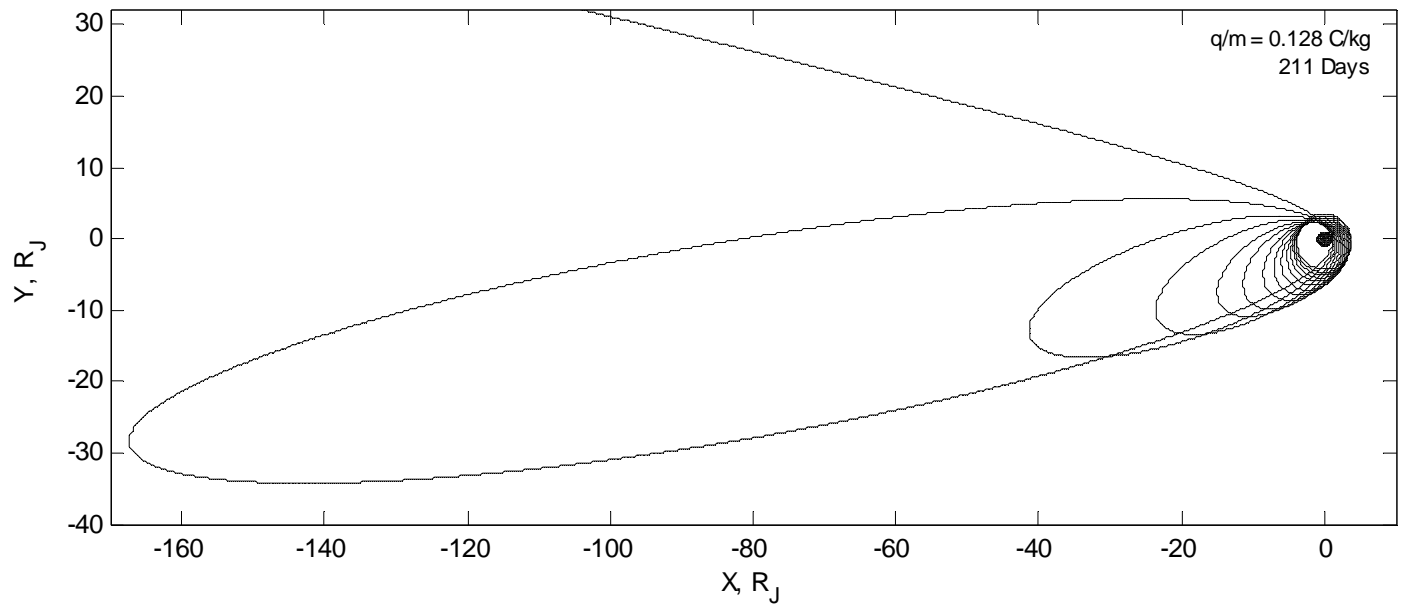

Figure 6.8. Minimum charge-to-mass ratio to capture on approach ( $\mathrm{q} / \mathrm{m}=0.128 \mathrm{C} / \mathrm{kg})$ simulated for 211 days with high-order model. 


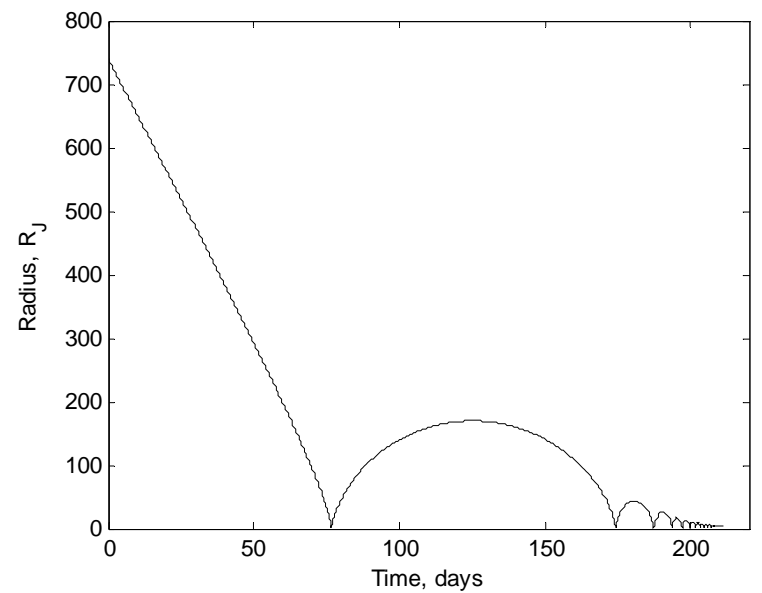

Figure 6.9. Radius time-history for bang-off actuated charge-to-mass spacecraft with parabolic approaches.

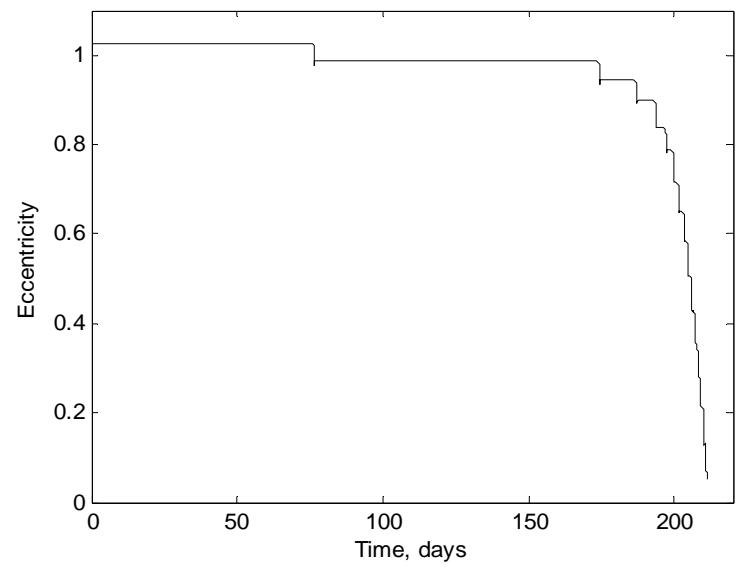

Figure 6.10. Eccentricity time-history for bang-off actuated charge-to-mass spacecraft with parabolic approaches.

\subsection{Feasibility}

In an effort to evaluate the feasibility of developing a LAO subsystem for Jovian orbit insertion, we adapt a previous LAO subsystem designed for Earth-LEO maneuvers [9]. Maximizing $q / m$ requires the balance of charge, mass, and power costs. Net charge is the product of the system's self-capacitance $C$ and the potential $V$ 


$$
q=C V
$$

Self-capacitance describes the number of electrons that can reside on the surface of a conductor in the plasma. Conductor geometries with high $C / m$ ratios are therefore favorable. This ratio is roughly associated with surface area-per-mass $A / m$. Likewise, it is favorable to maximize the system's potential. However, increasing surface area or potential increases the ambient plasma discharge currents. These currents must be countered, for example through the expulsion of charged particles via an ion or electron beam.

One candidate solution uses a negatively charged EDT-like architecture [9]. Here, a tether is used as a means of holding charge, rather than conducting current. Tether designs have cross-sectional dimensions that are much smaller than typical plasma electron gyroradii. As a result, they are subject to orbital-motion-limited (OML) discharging, which has characteristically lower currents than thermal or ram discharging mechanisms [20]. Further, they have a large vacuum $C / m$ ratio. In plasma, their capacitance is improved by the presence of a sheath of oppositely charged particles. Our candidate subsystem consists of a group of charged long, thin filaments that are grouped to form a cage-like structure.

We modify the LAO spacecraft design of Ref. [9] to account for sample Jovian plasma parameters near the planet $\left(\sim 1-3 \mathrm{R}_{\mathrm{J}}\right)$ at the equator: $1 \mathrm{~m}$ Debye length, $10 \mathrm{eV}$ temperature, and $\mathrm{S}^{+}$as dominant ion species $[21,15,16]$. Given these values, a radioisotope thermal generator can produce sufficient power to achieve an approximate $q / m$ of $0.03 \mathrm{C} / \mathrm{kg}$ for a $50 \mathrm{~kg}$ payload. The filament cage structure consists of 7000 filaments, each $20 \mathrm{~km}$ long and charged to $40 \mathrm{kV}$ using $2 \mathrm{~kW}$. This power requirement is orders of magnitude lower than similar EDT maneuvers but must be supplied by an on-board power source. 
This sample $q / m$ value is only one fourth the target for the EDT comparison, and the subsystem's large dimensions present a set of deployment challenges similar to those facing an EDT equipped spacecraft. However, this design does suggest that the minimum $q / m$ for Jovian orbit insertion (hundredths of a $\mathrm{C} / \mathrm{kg}$ ) is likely achievable, and therefore those captures illustrated in Figure 6.a and 6.b may be possible.

\subsection{Conclusions}

We conclude that the Lorentz force can be used to capture a spacecraft into an orbit about Jupiter and circularize that orbit if the spacecraft can achieve a controllable charge-to-mass ratio as low as hundredths of a Coulomb per kilogram. As the available $\mathrm{q} / \mathrm{m}$ increases, the spacecraft's ability to capture and circularize the orbit improves. At $0.1 \mathrm{C} / \mathrm{kg}$, the spacecraft can replicate a candidate electrodynamic tether

maneuver that places the spacecraft in a circular Jovian orbit at Europa with a comparable mission duration. In part due to the dependence of the Lorentz force on radial distance, the success of capture attempts depends largely on the orbit's perijove altitude. Our simple feasibility study suggests that spacecraft designs that achieve sufficient capture $q / m$ ratios introduce difficult challenges but are likely possible.

\subsection{Acknowledgments}

This work was supported by the NASA Institute for Advanced Concepts. 


\section{REFERENCES}

[1] Grün, E., Krüger, H., and Graps, A., "Galileo Observes Electromagnetically Coupled Dust in the Jovian Magnetosphere," Journal of Geophysical Research, Vol. 103, No. E9, 1998, pp. 20,011-20,022.

[2] Colwell, J.E., Horanyi, M., and Grun, E., "Capture of Interplanetary and Interstellar Dust by the Jovian Magnetosphere," Science (New York, N.Y.), Vol. 280, No. 5360, 1998, pp. 88-91.

[3]Khurana, K. K., Kivelson, M.G., Vasyliunas, V.M., Krupp, N., Woch, J., Lagg, A., Mauk, B. H., and Kurth, W.S., "The Configuration of Jupiter’s Magnetosphere,” Jupiter: The Planet, Satellites and Magnetosphere, Cambridge University Press, 2004, pp. 593-616

[4] Peck, M., "Prospects and Challenges for Lorentz-Augmented Orbits," Proceedings of the AIAA Guidance, Navigation, and Control Conference, August 15-18, 2005, AIAA-2005-5995.

[5] Streetman, B. and Peck, M., "Gravity-Assist Maneuvers Augmented by the Lorentz Force," AIAA Guidance, Navigation and Control Conference and Exhibit, Hilton Head, South Carolina, Aug. 20-23, 2007, AIAA-2007-6846.

[6] Acuna, M., Behannon, K., and Connerney, J., "Jupiter's Magnetic Field and Magnetosphere," Physics of the Jovian Magnetosphere, Vol. 83, No. 26611, 1983, pp. 10-91.

[7] Streetman, B., and M. Peck, "New Synchronous Orbits Using the Geomagnetic Lorentz Force," Journal of Guidance, Control, and Dynamics, Vol. 30, No. 6, 2007, pp. 1677-1690, AIAA-2006-6804.

[8] Bellman, R., Glicksberg, I., and Gross, O., "On the 'Bang-Bang' Control Problem," Quarterly of Applied Math, Vol. 14, 1956, pp 11-18.

[9] Streetman, B. and Peck, M., "A General Bang-Bang Control Method for Lorentz Augmented Orbits," AAS Spaceflight Mechanics Meeting, Galveston, TX, Jan 2731, 2008. AAS 08-111.

[10] Kirk, D.E., "Optimal Control Theory: An Introduction," Courier Dover Publications, 2004, pp. 240-259. 
[11] Atchison, J., Streetman, B., and M. Peck, "Prospects for Lorentz Augmentation in Jovian Captures," Proceedings of the AIAA Guidance, Navigation, and Control Conference, August 21-24, 2006, AIAA-2006-6596.

[12] Williams, P., "Periodic Solutions of Electrodynamic Tethers under Forced Current Variations," Proceedings of the AIAA Guidance, Navigation, and Control Conference, August 21-24, 2006, AIAA-2006-6765.

[13] Penzo, P. A., “A Survey of Tether Applications to Planetary Exploration,” Advances in the Astronautical Sciences, Vol. 62, 1986, pp. 71-88.

[14] Hammond, W. E., Freeman, S. E., Nave, M. J., and Rupp, C. C., “Jovian Electrodynamic Tether Experiment,” Aerospace Sciences Meeting, January 7-10 1991, AIAA 91-426.

[15] D.L. Gallagher, L. Johnson, J. Moore, F. Bagenal, "Electrodynamic Tether Propulsion and Power Generation at Jupiter," NASA, Marshall Space Flight Center; National Technical Information Service, distributor, 1998.

[16] Talley, C., Moore, J., and Gallagher, D., "Propulsion and Power from a Rotating Electrodynamic Tether at Jupiter," AIAA, Aerospace Sciences Meeting and Exhibit, 2000, AIAA-2000-326.

[17] Sanmartín, J., and Lorenzini, E.C., "Exploration of Outer Planets Using Tethers for Power and Propulsion”, Journal of Propulsion and Power, Vol. 21, No. 3, 2005, pp. 573-576.

[18] Sanmartín, J., Charro, M., Lorenzini, E.C., Garrett, H.B., Bombardelli, C., and Bramanti, C., "Electrodynamic Tether at Jupiter-I: Capture Operation and Constraints”, IEEE Transactions on Plasma Science, Vol. 36, No. 5, 2008, pp. 2450-2458.

[19] Khurana, K.K., "Euler Potential Models of Jupiter's Magnetospheric Field," Journal of Geophysical Research, Vol. 102, No. A6, June 1997, pp. 11,29511,306 .

[20] Sanmartín, J. R., Martinez-Sanchez, M., and Ahedo, E., "Bare Wire Anodes for Electrodynamic Tethers,” Journal of Propulsion and Power, Vol. 9, June 1993, pp. 353-360. 
[21] Bagenal, F., "Empirical Model of the Io Plasma Torus: Voyager Measurements," Journal of Geophysical Research, Vol. 99, No. A6, June 1994, pp. 11,04311,062 . 


\section{CHAPTER 7}

\section{LORENTZ ACCELERATIONS IN THE EARTH FLYBY ANOMALY*}

\subsection{Abstract}

Mission engineers have detected an unexpected anomaly on six spacecraft during low-altitude gravity-assist maneuvers around Earth. This Earth flyby anomaly involves an acceleration that, to date, researchers cannot account for based on known forces or errors in measurement or modeling. This paper evaluates Lorentz accelerations associated with spacecraft electrostatic charging as a possible explanation for the Earth flyby anomaly. This analysis does not explicitly address plasma physics but instead bases its conclusions on fundamental six-state flight dynamics. The analysis focuses on the Near Earth Asteroid Rendezvous (NEAR) spacecraft because it exhibited the largest anomalous error with the smallest estimated residuals. The analysis takes the form of a boundary-value problem in which vectordisturbance time histories are found numerically through nonlinear optimization methods. The analysis identifies the unknown, but required, acceleration based on a model of the Lorentz-force interaction. The algorithm cannot converge on a solution that fully reproduces the anomalous error in all six orbital states. It is unlikely, based on this analysis, that Lorentz forces cause the flyby anomaly.

\footnotetext{
${ }^{*}$ Reproduced with permission from the Journal of Guidance, Control, and Dynamics To be published Spring 2010.
} 


\subsection{List of Symbols}

\begin{tabular}{|c|c|c|}
\hline$a_{i}$ & $=$ & $i^{\text {th }}$ cosine coefficient in a Fourier series \\
\hline$b_{i}$ & $=$ & $i^{\text {th }}$ sine coefficient in a Fourier series \\
\hline $\boldsymbol{B}$ & $=$ & magnetic field \\
\hline$\delta_{i}$ & $=$ & declination angle of incoming spacecraft velocity vector \\
\hline$\delta_{o}$ & $=$ & declination angle of outgoing spacecraft velocity vector \\
\hline$E$ & $=$ & eccentric anomaly \\
\hline$e$ & $=$ & eccentricity \\
\hline$\varepsilon$ & $=$ & orbital energy \\
\hline$F_{L}$ & $=$ & Lorentz force \\
\hline$\phi$ & $=$ & colatitudes angle, measured from the $+z$-axis \\
\hline$\Gamma(t)$ & $=$ & truncated Fourier series approximation of a function of time \\
\hline$g_{i}$ & $=$ & perturbation accelerations projected onto $\hat{\boldsymbol{\imath}}$ \\
\hline$H(t)$ & $=$ & Heaviside step function \\
\hline$h$ & $=$ & orbital angular momentum \\
\hline$i$ & $=$ & inclination \\
\hline $\boldsymbol{J}$ & $=$ & vector cost function \\
\hline$K$ & $=$ & unitless constant \\
\hline$M$ & $=$ & mean anomaly \\
\hline$m$ & $=$ & spacecraft mass \\
\hline$\mu$ & $=$ & geocentric gravitational constant \\
\hline$v$ & $=$ & true anomaly \\
\hline$\Pi(t)$ & $=$ & boxcar shape function \\
\hline$q$ & $=$ & net electrostatic charge \\
\hline$r$ & $=$ & spacecraft position vector with magnitude $r$ and direction $\hat{\boldsymbol{r}}$ \\
\hline
\end{tabular}




$$
\begin{aligned}
& \boldsymbol{\rho}^{0}=\text { observed state errors due to anomaly } \\
& t=\text { time } \\
& \tau=\text { time constant of basis function } \\
& \theta=\text { azimuthal angle, measured from the }+\mathrm{x} \text {-axis } \\
& v \quad=\text { spacecraft velocity vector } \\
& v_{\text {rel }}=\text { spacecraft velocity relative to } \boldsymbol{B} \\
& v_{\text {inf }}=\text { hyperbolic excess velocity } \\
& \boldsymbol{W}=\text { cost function weighting matrix } \\
& \Omega \quad=\quad \text { longitude of the ascending node } \\
& \omega=\text { argument of perigee } \\
& \omega_{B}=\text { vector spin rate of the magnetic field } \\
& \boldsymbol{X}=\text { spacecraft orbital state vector } \\
& X_{2}=\quad \text { simulated charged spacecraft state after close approach } \\
& \boldsymbol{X}_{2}{ }^{0}=\text { predicted spacecraft state after close approach } \\
& \boldsymbol{X}_{2}{ }^{*}=\text { first observed spacecraft state after close approach } \\
& x, y, z=\text { Cartesian coordinates aligned with a geocentric equatorial inertial } \\
& \text { system }
\end{aligned}
$$




\subsection{Introduction}

Nearly every deep-space mission of the past thirty years has used a gravity-assist maneuver, or flyby, to achieve low-cost $\Delta v$. Gravity assists enabled Voyager I to depart the Solar System, Apollo 13 to return to Earth safely from the Moon, Galileo to tour the Jovian moon system, and Ulysses to view the Sun's polar regions. These maneuvers capitalized on the physics of three-body dynamics to transfer energy and momentum between a planet and a spacecraft in a heliocentric reference frame. These physics have been understood in terms of the restricted three-body problem for nearly two centuries.

It was therefore surprising when, in 1990, engineers at the Jet Propulsion Laboratory observed an energy change in the Galileo spacecraft's Earth gravity assist (EGA) maneuver that could not be explained.[1,2] At the spacecraft's close approach

to Earth, radar tracking stations were unable to slew quickly enough to follow Galileo's trajectory. When the acquisition of radar data resumed, tracking indicated that the spacecraft had experienced an unexplained acceleration that resulted in an orbit different from what had been predicted. After detecting this anomaly, researchers began a systematic study to identify what has come to be known as the flyby anomaly and identify its potential causes.

Since that initial observation, mission engineers have observed five other anomalous EGAs. Table 7.1 reproduces relevant values for the anomalous EGAs [2]. The details vary among these gravity-assist maneuvers, but they all share an unexplained acceleration. Though the anomalous acceleration produces a change in all of the orbital states, it is often described as a change in the hyperbolic excess velocity $v_{\text {inf. }}$ In the six known cases, $v_{\text {inf }}$ has been observed to both increase and decrease with magnitudes on the order of mm/s. Four additional EGAs with high- 
altitude close approaches have not shown anomalous energy changes. So, some infer a correlation with distance..$^{\dagger}$

Table 7.1. Anomalous Flyby Parameters [2]

\begin{tabular}{lcccccc}
\hline \hline Spacecraft & Galileo & Galileo & NEAR & Cassini & Rosetta & M'GER \\
\hline Date of Flyby & $12 / 08 / 90$ & $12 / 08 / 92$ & $01 / 23 / 98$ & $08 / 18 / 99$ & $03 / 04 / 05$ & $08 / 02 / 05$ \\
Mass, kg & 2497 & 2497 & 730 & 4612 & 2895 & 1086 \\
Altitude of Perigee, km & 960 & 303 & 539 & 1175 & 1956 & 2347 \\
Inclination, deg & 142.9 & 138.7 & 108.0 & 25.4 & 144.9 & 133.1 \\
Turn Angle, deg & 47.7 & 51.1 & 66.9 & 19.7 & 99.3 & 94.7 \\
Velocity at Perigee, $\mathrm{km} / \mathrm{s}$ & 13.740 & 14.080 & 12.739 & 19.026 & 10.517 & 10.389 \\
$v_{\text {inf }}, \mathrm{km} / \mathrm{s}$ & 8.949 & 8.877 & 6.851 & 16.010 & 3.863 & 4.056 \\
Anomalous $\Delta v_{\text {inf }}, \mathrm{mm} / \mathrm{s}$ & 3.92 & -4.6 & 13.46 & -2 & 1.80 & 0.02 \\
Error Bound $\Delta v_{\text {inf }}, \mathrm{mm} / \mathrm{s}$ & 0.3 & 1.0 & 0.01 & 1 & 0.03 & 0.01 \\
\hline \hline
\end{tabular}

Researchers have ruled out measurement errors as a cause of the flyby anomaly and have determined that the acceleration is a physical phenomenon: a variety of instruments and algorithms have detected the acceleration for the different spacecraft and orbits [2]. In addition, radar measurements have confirmed the anomaly using both Doppler and time-of-flight range data. Researchers have proposed and evaluated a wide range of possible causes for the flyby anomaly[3,4,5]. Antreasian and Guinn have shown that the anomaly cannot be produced by a conservative field only and the unknown acceleration may vary in time [6].

\footnotetext{
${ }^{\dagger}$ Personal communication with Dr. John D. Anderson on January 23, 2009.
} 
Anderson and his colleagues recently reported that the magnitude of the anomaly correlates with the cosine of the declination of the spacecraft's incoming $\delta_{i}$ and outgoing $\delta_{o}$ velocity vectors [2]

$$
\frac{\Delta v_{i n f}}{v_{i n f}}=K\left(\cos \delta_{i}-\cos \delta_{o}\right)
$$

The unitless constant $K$ is empirically calculated to match the known data points, though it can be related to known Earth constants. Two papers explore Eq. 1 as a consequence of an unknown force field. Hasse, Birsin, and Hähnel conclude that any candidate force field would contain a dependence on the velocity of the spacecraft [7]. Lewis finds that a quadrupole scalar potential field could produce this fit, and would relate to a coupling of kinetic energies between the Earth and spacecraft [8].

Since Anderson and his colleague's finding, a variety of specific explanations have been proposed. These address reassessments of general and special relativity $[9,10,11,12,13]$, the number of dimensions of space [14], dark matter somehow bound to Earth [15,16], the Casimir effect [17,18], and the concept of privileged frames [19]. To date, the research community has not yet accepted any of these studies as an adequate explanation for the flyby anomaly.

Five specific observations motivate evaluation of Lorentz forces associated with spacecraft charging as a source of the anomaly. The Lorentz force is based on wellunderstood physics: it has been observed in the orbital dynamics of dust particles at Jupiter [20,21,22] and Saturn [23,24]. The Lorentz force is non-conservative, transferring energy between the Earth's rotating magnetic field and the charged spacecraft's orbit25. This transfer can either increase or decrease the energy of a spacecraft's orbit. The Lorentz force is distance-dependent, varying with $r^{-7 / 2}$. 
Finally, simulated Lorentz maneuvers at Earth show a strong correlation between declination and energy change [26].

In their review of a number of unexplained physical phenomena, Lammerzahl, Preuss, and Dittus address the problem of the Earth flyby anomaly. In a brief paragraph, the authors dismiss the Lorentz force using simple order-of-magnitude estimates [3]:

"In a recent study of charging of the LISA test masses [27] the charging has been estimated by $10^{-10} \mathrm{C}$. So, for the whole satellite it might be a conservative assumption that the charge is less than $\mathrm{Q} \leq 10^{-7} \mathrm{C}$. A satellite of $1 \mathrm{t}$ carrying a charge $\mathrm{Q}$ and moving with $\mathrm{v}=30 \mathrm{~km} / \mathrm{s}$ in the magnetic field of the Earth which is of the order $0.2 \mathrm{G}$ will experience an acceleration $10^{-8} \mathrm{~m} / \mathrm{s}^{2}$ far below the observed effect."

Their analysis rules out the Lorentz force on the basis of a priori estimates of spacecraft charge. In contrast, the objective of the present work is to investigate the problem without presupposing a certain charge magnitude. Instead, it is based on a full vector-mechanics representation of the physics. Furthermore, previous research has explored the possibility of generating electrostatic charge on spacecraft to achieve novel spacecraft maneuvers, [23,24,28,29] including augmented flybys [30]. This work has shown that the Lorentz force can generate meaningful acceleration on a spacecraft. The present study begins from first principles to investigate the possibility that a spacecraft with any electrostatic charge can recreate these anomalous behaviors, regardless of magnitude, sign or variation in time. Put simply, the spacecraft charge is an output of this analysis, not an input. 
This analysis addresses this question in the context of a test case, the Near Earth Asteroid Rendezvous (NEAR) spacecraft, using original data. This spacecraft demonstrated the greatest anomaly with the lowest error residuals of all the examples, and it is therefore the best candidate to address. Demonstrating that Lorentz acceleration can explain NEAR's anomalous orbit time-history is necessary, but not sufficient, evidence for explaining the others.

\subsection{Modeling and Analysis}

A spacecraft with an electrostatic charge $q$, moving with velocity $\boldsymbol{v}_{r e l}$ relative to a magnetic field $\boldsymbol{B}$, experiences the Lorentz force $\boldsymbol{F}_{\boldsymbol{L}}$

$$
\boldsymbol{F}_{\boldsymbol{L}}=q \boldsymbol{v}_{r e l} \times \boldsymbol{B} .
$$

For a rotating magnetic field

$$
v_{r e l}=\dot{\boldsymbol{r}}-\omega_{B} \times \boldsymbol{r},
$$

$\dot{r}$ is the vector time derivative of the spacecraft's position $\boldsymbol{r}$ (with magnitude $r$ and direction $\hat{r}$ ) relative to the system's barycenter in a Newtonian frame, and $\omega_{B}$ is the angular velocity of the magnetic field.

The spacecraft's orbital state determines the value for every term in these expressions except for the scalar $q$. Thus, the direction of the Lorentz force is confined to a one-dimensional manifold corresponding to varying charge magnitude. Figure 7.1 shows the line-of-action for the blackout time of NEAR at three-minute increments along the orbital path. The spacecraft approaches Earth from the $+\mathrm{z}$ direction and makes its closest approach above the Middle East. The direction of $\boldsymbol{v}_{\boldsymbol{r} e l} \mathrm{x} \boldsymbol{B}$ is nearly constant prior to the close approach and again after the close approach. At closest 
approach, $\boldsymbol{v}_{\boldsymbol{r e l}} \times \mathrm{B}$ lies in a predominantly cross-track direction, after which it rotates to an along-track direction. Figure 7.2 shows the magnitude of $\boldsymbol{v}_{\boldsymbol{r} e l} \times \boldsymbol{B}$ versus time over the data blackout period, in which the vector magnitude is normalized by the maximum value. The magnitude is largest near close approach, where both $\left|\boldsymbol{v}_{\text {rel }}\right|$ and $|\boldsymbol{B}|$ are maximized. This magnitude quickly drops off, leaving only a one-hour window in which the Lorentz force can be of any significance.

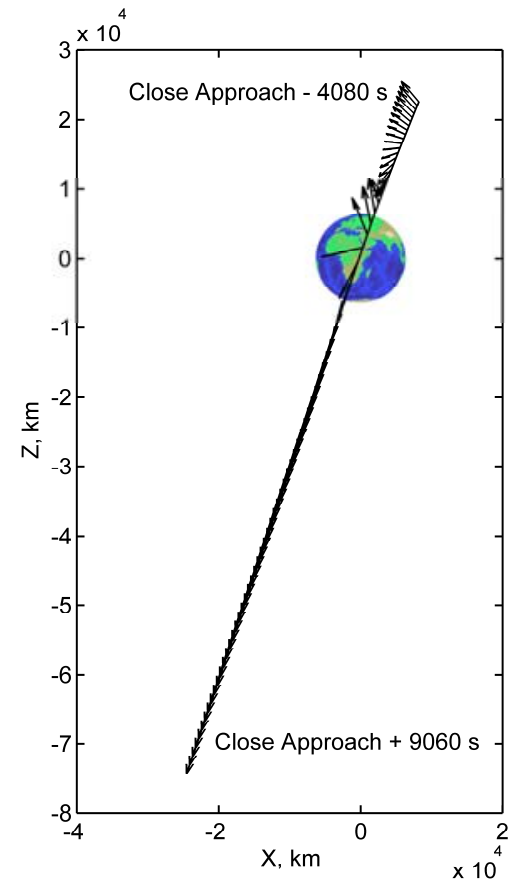

a.)

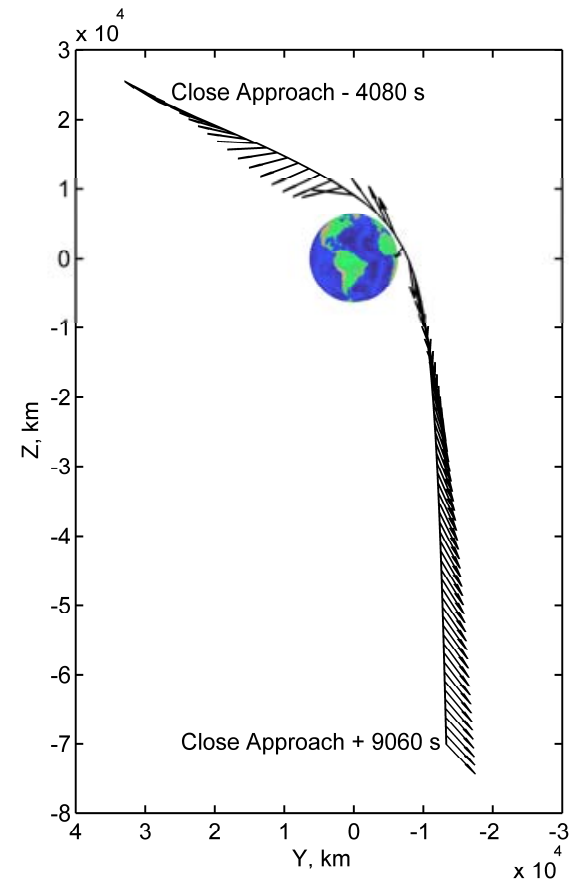

b.)

Figure 7.1. The direction of $\left(v_{r e l} \times B\right)$ for the NEAR EGA during the data-blackout period shown from a.) the Eastern Hemisphere b.) the Western Hemisphere. 


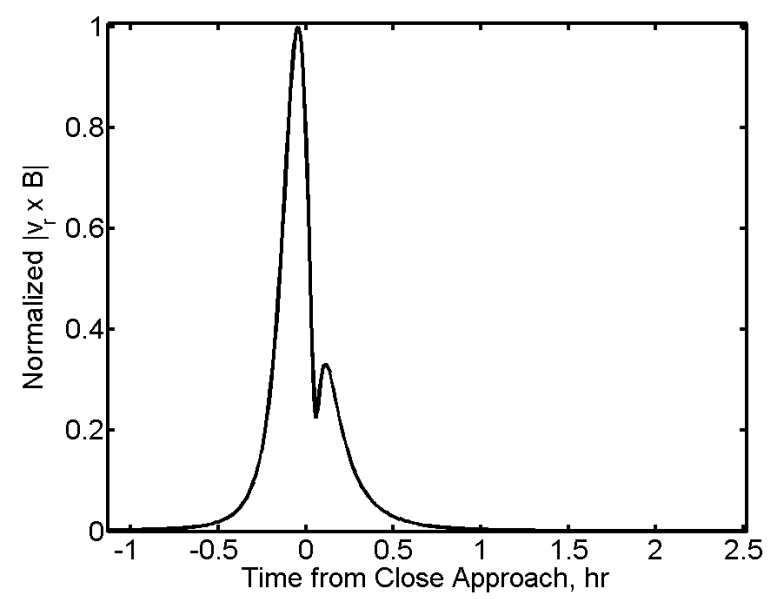

Figure 7.2. The normalized magnitude of $\left(v_{r e l} \times B\right)$ for the NEAR EGA during radar blackout.

The following two-body equations of motion represent the Lorentz force in terms of spherical coordinates:

$$
\begin{gathered}
\ddot{r}=r \dot{\theta}^{2} \sin ^{2} \phi+r \dot{\phi}^{2}-\frac{\mu}{r^{2}}+g_{r}+\left(\frac{\boldsymbol{F}_{L}}{m} \cdot \hat{\boldsymbol{r}}\right) \\
r \ddot{\phi}=-2 \dot{r} \dot{\phi}+r \dot{\theta}^{2} \sin \phi \cos \phi+g_{\phi}+\left(\frac{\boldsymbol{F}_{L}}{m} \cdot \widehat{\boldsymbol{\phi}}\right) \\
r \sin \phi \ddot{\theta}=-2 \dot{r} \dot{\theta} \sin \phi-2 r \dot{\phi} \dot{\theta} \cos \phi+g_{\theta}+\left(\frac{\boldsymbol{F}_{L}}{m} \cdot \widehat{\boldsymbol{\theta}}\right),
\end{gathered}
$$

where the coordinates and axes are given in Figure 7.3. The terms $g_{r}, g_{\phi}$, and $g_{\theta}$ are perturbation accelerations along each axis. The model for the present analysis includes accelerations due to high-order gravitational terms (100 x 100 EGM96), nbody direct and indirect effects (Sun, Moon, Jupiter, and Venus), solar radiation pressure, relativity (point-mass and Lense-Thirring effects), and atmospheric drag. Accelerations associated with Earth albedo, Earth infrared, ocean tides, indirect oblateness, moon oblateness, and the remaining planets are negligible. (For a review of these effects for the NEAR and Galileo EGAs, see Ref. 5.) In spite of these omissions, the model recreates the published value of anomalous $\Delta v_{i n f}$ to within 
0.01\%. The magnetic field $\boldsymbol{B}$ is modeled with a $10 \times 10$ spherical harmonic representation of the International Geomagnetic Reference Field for 1995. In each equation (3), $\frac{F_{L}}{m}$ represents the acceleration imparted by the Lorentz force.

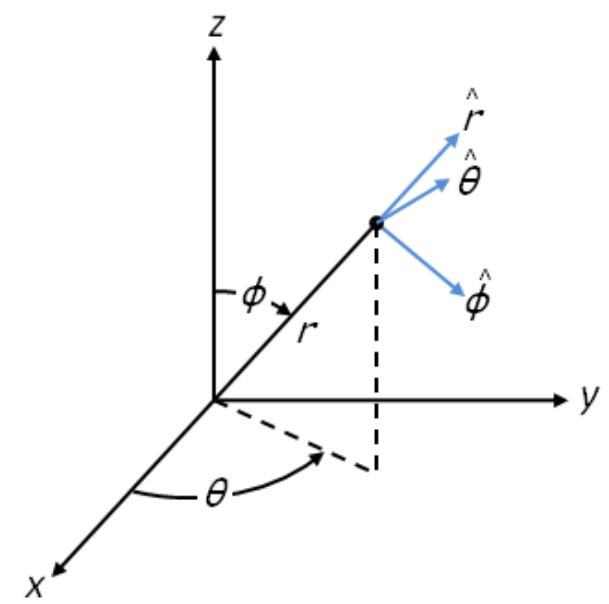

Figure 7.3. Spherical coordinates and axes.

The scalar hyperbolic excess energy is

$$
v_{i n f}^{2}=\boldsymbol{v} \cdot \boldsymbol{v}-\frac{2 \mu}{r}
$$

A complete evaluation of the anomaly addresses the transition of six orbital states $\boldsymbol{X}$, during the flyby rather than simply representing the difference in energy, a mere scalar that cannot represent the full subtlety of the problem. $\boldsymbol{X}$ can be expressed, for example, in Cartesian coordinates taken in the Geocentric Equatorial Inertial (GEI) frame $[x, y, z, \dot{x}, \dot{y}, \dot{z}]$, spherical coordinates as described in the equations of motion $[r, \phi, \theta, \dot{r}, \dot{\phi}, \dot{\theta}]$, or Earth-centered classical orbital elements $[a, e, i, \Omega, \omega, v]$. Table 7.2 shows the observed anomalous change in each of these representations of the six states.

Perturbation techniques can map the changes associated with the Lorentz force throughout NEAR's orbit into these various coordinates. Then, general perturbation 
equations shown in Equation 9-24 of Ref. 31, transform the change into orbital elements. Figure 7.4 shows the accumulated changes for nine such coordinates for a constant positive $q / m=+1 \mathrm{mC} / \mathrm{kg}$, an arbitrary example. (The polarity of $q / m$ appears linearly in the calculation of $F_{L}$, so a negatively charged spacecraft produces the opposite of the changes observed in Figure 7.4.) Three elements $(\Omega, \omega, M)$ show monotonic change throughout the flyby. Comparing these results with Table 7.2 leads to the conclusion that the charge-to-mass ratio of the spacecraft must be negative at some point to produce the observed anomaly. These results offer no further insight beyond this single observation; one cannot rule out the Lorentz force solely on the basis of a single constant charge.

Table 7.2. Polarity and Magnitude of Anomalous State Change for NEAR

\begin{tabular}{cccccc}
\hline \multicolumn{2}{c}{$\begin{array}{c}\text { GEI Cartesian } \\
\text { Coordinates }\end{array}$} & \multicolumn{2}{c}{$\begin{array}{c}\text { GEI Spherical } \\
\text { Coordinates }\end{array}$} & \multicolumn{2}{l}{ Orbital Elements } \\
\hline$x$ & $+10^{2} \mathrm{~m}$ & $r$ & $-10^{2} \mathrm{~m}$ & $\varepsilon$ & $+10^{2} \mathrm{~J} / \mathrm{kg}$ \\
$y$ & $-10^{3} \mathrm{~m}$ & $\phi$ & $-10^{-7} \mathrm{rad}$ & $h$ & $+10^{5} \mathrm{~m}^{2} / \mathrm{s}$ \\
$z$ & $+10^{2} \mathrm{~m}$ & $\theta$ & $+10^{-6} \mathrm{rad}$ & $e$ & $+10^{-6}$ \\
$\dot{x}$ & $-10^{-3} \mathrm{~m} / \mathrm{s}$ & $\dot{r}$ & $+10^{-2} \mathrm{~m} / \mathrm{s}$ & $i$ & $-10^{-6} \mathrm{rad}$ \\
$\dot{y}$ & $-10^{-3} \mathrm{~m} / \mathrm{s}$ & $\dot{\phi}$ & $+10^{-10} \mathrm{rad} / \mathrm{s}$ & $\Omega$ & $-10^{-6} \mathrm{rad}$ \\
$\dot{z}$ & $-10^{-2} \mathrm{~m} / \mathrm{s}$ & $\dot{\theta}$ & $+10^{-11} \mathrm{rad} / \mathrm{s}$ & $\omega$ & $-10^{-8} \mathrm{rad}$ \\
& & & & $v$ & $-10^{-6} \mathrm{rad}$ \\
& & & & $M$ & $+10^{-5} \mathrm{rad}$ \\
& & & & & \\
\hline \hline
\end{tabular}



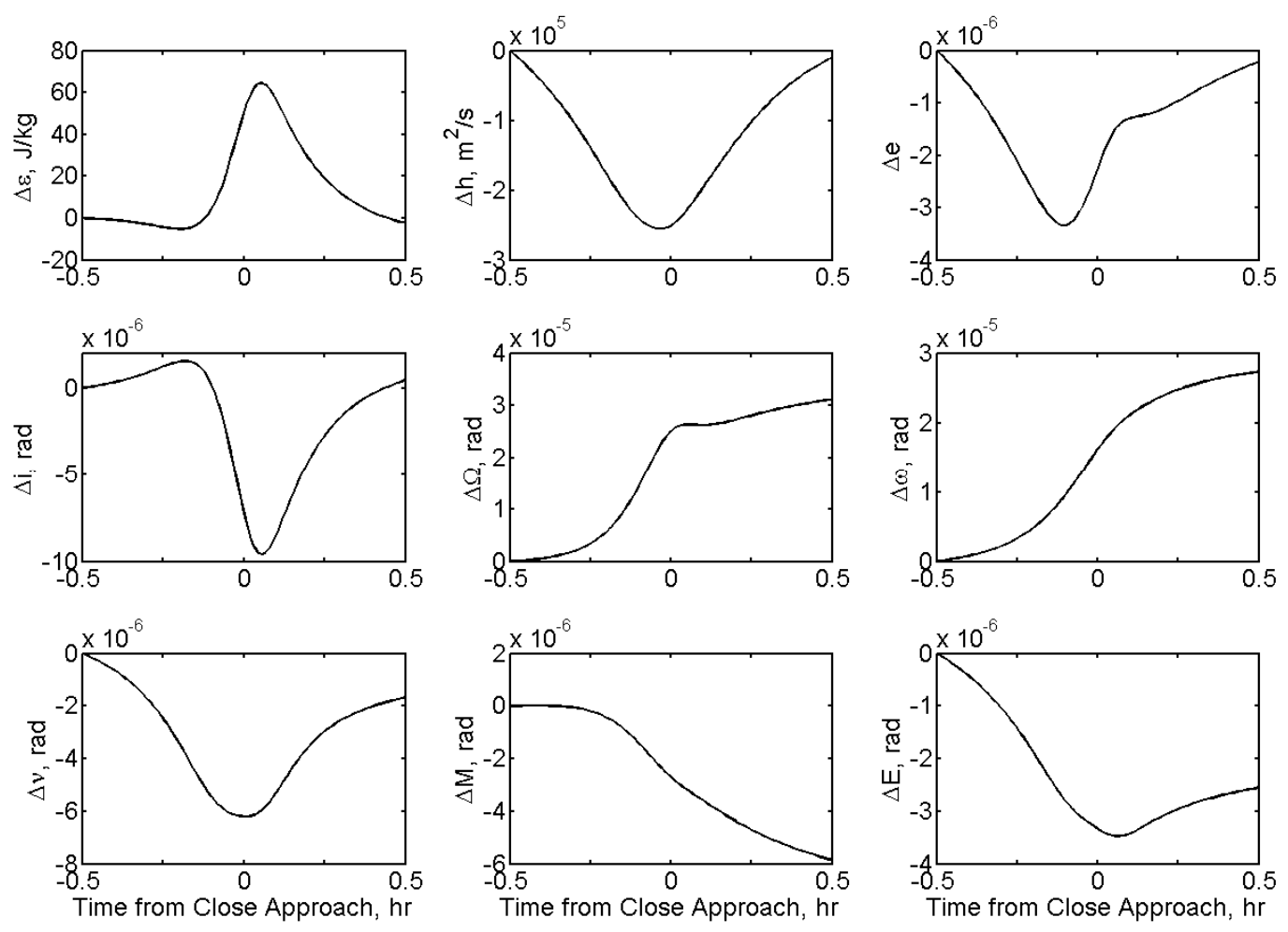

Figure 7.4. Accumulated change in orbital elements associated with a constant positive charge-to-mass ratio of $1 \mathrm{mC} / \mathrm{kg}$ for a one-hour window around closest approach.

\subsection{Numerical Approach}

Since the results of the perturbation analysis are inconclusive, this research turns to numerical optimization methods for nonlinear systems. In this context, the anomaly is treated as an optimization problem with known boundary-values; seeking to solve for a $q(t)$ that can evolve from the last known state before the radar blackout $\boldsymbol{X}_{\boldsymbol{I}}$ to the first known state after the radar blackout $\boldsymbol{X}_{2}{ }^{*}$. Figure 7.5 illustrates these states with exaggerated orbits. $\boldsymbol{X}_{2}{ }^{0}$ is the predicted state, found by propagating $\boldsymbol{X}_{1}$ forward in time without any anomalous acceleration. The optimizer is meant to find a charge time 
history using an algorithm that minimizes a cost associated with the anomalous state errors. For this analysis, the cost is the weighted vector difference between the known post-encounter state $\boldsymbol{X}_{2}{ }^{*}$ and the post-encounter state $\boldsymbol{X}_{2}$, simulated with a non-zero $q(t)$ time history:

$$
\begin{gathered}
J=W\left[X_{2}^{*}-X_{2}\right], \\
J=\|J\| .
\end{gathered}
$$

The weighting matrix $\boldsymbol{W}$ is diagonal with coefficients consisting of the inverse of the absolute value of the errors $\rho^{0}$ :

$$
\begin{gathered}
\rho^{0}=\boldsymbol{X}_{\mathbf{2}}^{*}-\boldsymbol{X}_{\mathbf{2}}^{0}, \\
\boldsymbol{W}=\frac{\mathbf{1}}{\sqrt{6}}\left(\operatorname{diag}\left|\rho^{0}\right|\right)^{-1} .
\end{gathered}
$$

This form normalizes the relative magnitudes of the components of $\boldsymbol{J}$, yielding a baseline cost for the uncharged spacecraft $J^{0}$ of 1.0. Values for $\rho^{0}$ are given in three coordinate systems in Table 7.2. Though the three systems-Cartesian, spherical, and classical orbital - are equivalent in the sense that the physics is independent of basis, $\boldsymbol{X}$ is expressed in a Cartesian-coordinate system for the cost modeling because its matrix expressions tend to be better conditioned than corresponding expressions in the other two systems. 


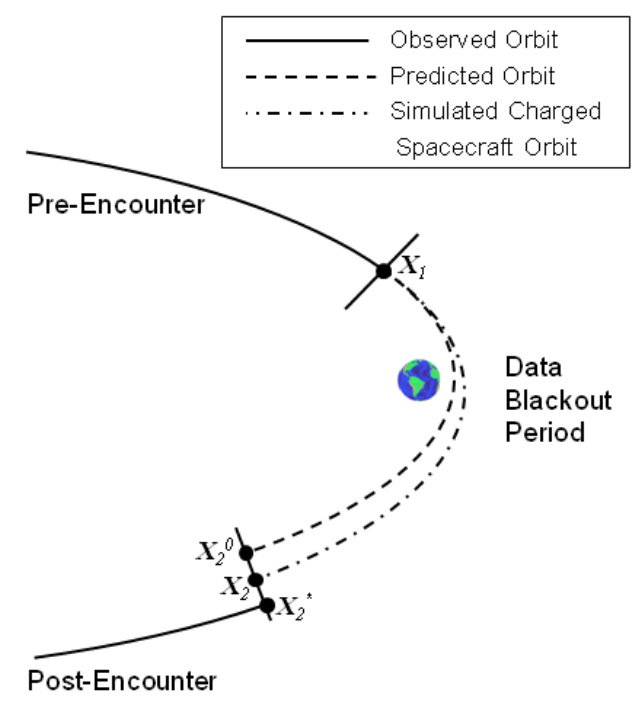

Figure 7.5. Diagram of state definitions.

A Fourier series serves as a basis to model the unknown acceleration. With enough terms, this basis can represent an arbitrary, continuous time history. Here, the series is truncated to some numerically manageable order, $n$. The Fourier series of an arbitrary function can be expressed as

$$
\begin{gathered}
\Gamma(t)=a_{0}+\sum_{i=1}^{n}\left[a_{i} \cos \left(\frac{2 \pi i}{\tau} t\right)+b_{i} \sin \left(\frac{2 \pi i}{\tau} t\right)\right] \\
a \in \mathbb{R}^{(n+1) \times 1} \\
b \in \mathbb{R}^{n \times 1} .
\end{gathered}
$$

The parameters $a_{i}$ and $b_{i}$ describe the shape of $\Gamma(t)$, and $\tau$ sets the length of the longest period. For an order $n$ Fourier series, the algorithm must optimize over $2 n+1$ coefficients. Figure 7.6 shows a sample of four functions that are approximated with a sixth order $(n=6)$ Fourier series. This shape function captures a line with constant slope, a Heaviside step, and a triangular wave, all with accuracy acceptable for this 
application. However, it is much less effective at modeling an impulse function. To accommodate this limitation this study also considers a boxcar basis function, which describes a single "on" period of a square wave

$$
\Pi(t)=c_{0}\left(H\left[t-t_{0}\right]-H\left[t-\left(t_{0}+\Delta t\right)\right]\right) .
$$

$H(t)$ is the Heaviside step function. The parameters $c_{0}$, $t_{0}$, and $\Delta t$ set the function's magnitude, start time, and pulse-width respectively. If $\Delta t$ is sufficiently small, this shape function can approximate a single impulse. These two shape functions model both the unknown acceleration and the proposed $q(t)$ time history.

MATLAB's nonlinear least-squares optimization function lsqnonlin offers good convergence and repeatability for this application. This function uses a LevenbergMarquardt line search method to estimate a local gradient and march toward a minimum cost $J$ by varying the coefficients $\left(a_{i}, b_{i}\right)$ that define $q(t)$. The change in step size defines the convergence. A threshold of $10^{-14}$ yields sufficiently accurate solutions in this analysis with acceptable computation time. There are no guarantees that the resulting minimum is global rather than simply local. So, each optimization is based on a large and varying set of initial guesses. 

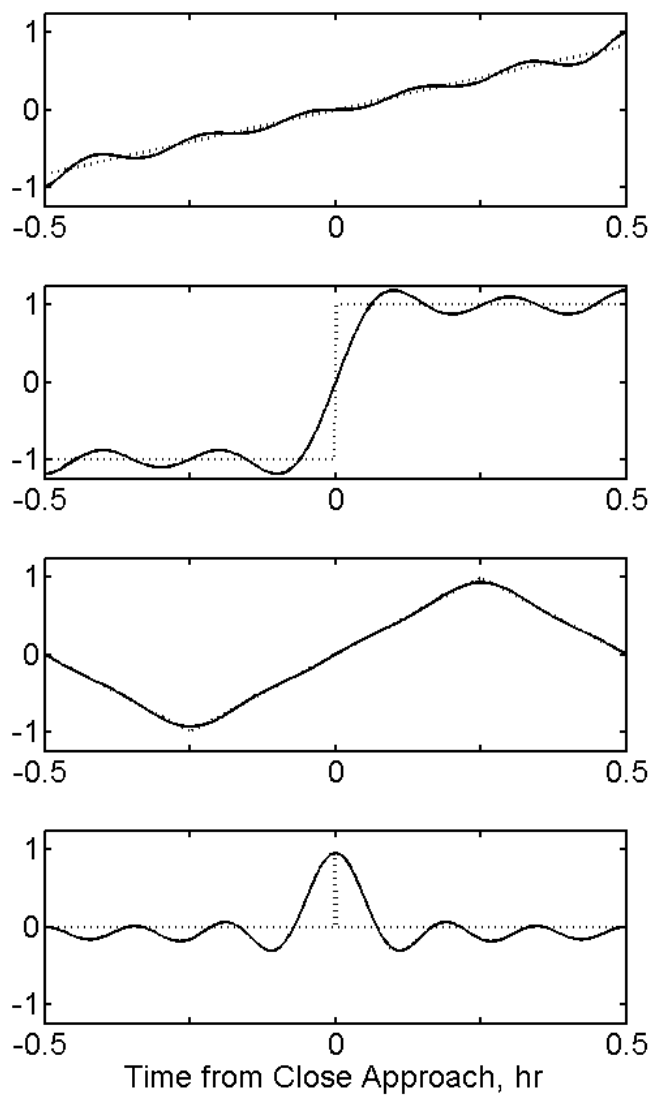

Figure 7.6. Sample functions (dotted lines) approximated by a $6^{\text {th }}$ order Fourier series (solid lines).

To verify this numerical approach, one can test the algorithm with a known acceleration time history. As an example, the uncharged orbit from $\boldsymbol{X}_{\boldsymbol{1}}$ through the data blackout period is simulated with and without the presence of the Moon's accelerations. $\boldsymbol{X}_{2}{ }^{*}$ becomes the post-encounter state accounting for the Moon and $\boldsymbol{X}_{2}{ }^{\boldsymbol{0}}$ the post-encounter state excluding the Moon. The algorithm then solves for the missing known acceleration given only the direction of the Moon and modeling the magnitude of the acceleration as an $8^{\text {th }}$-order Fourier series. This test mimics the proposed Lorentz simulation in which the direction of $\boldsymbol{v}_{\boldsymbol{r e l}} \times \boldsymbol{B}$ is known and the timevarying magnitude of $q(t)$ is unknown. 
The minimized cost $J=10^{-9}$ suggests that the algorithm modeled the missing acceleration associated with the Moon with an acceptable precision because such a cost indicates residual error in all states that is orders of magnitude lower than the observational error. Figure 7.7 shows the true and modeled magnitude of acceleration throughout the data blackout period. The true lunar acceleration is similar to a portion of a triangle wave. The Fourier series approximation oscillates around the actual acceleration, with errors that are most noticeable near the two endpoints and the inflection point. However, the fact that the cost is based on an integrated time history allows these small errors to occur without significant impact. A higher-order approximation reduces these errors at the cost of computation time.

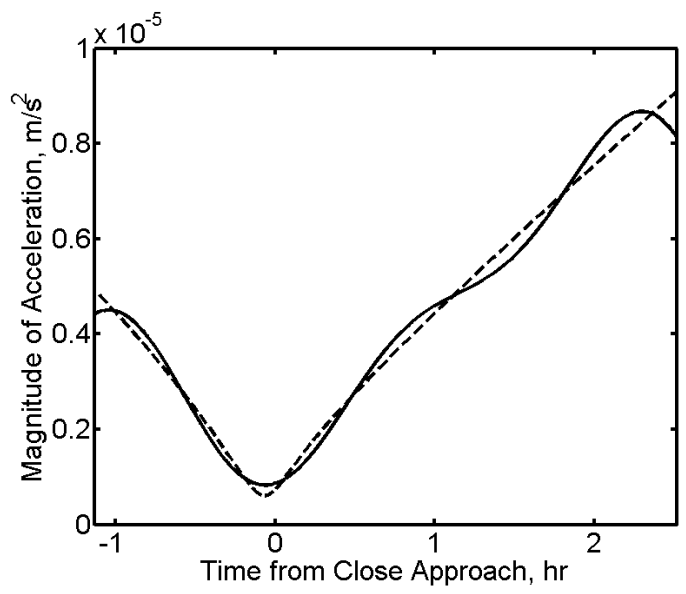

Figure 7.7. Magnitude of acceleration due to the Moon (dotted line), solved for by using the optimization algorithm (solid line).

\subsection{Results}

Prior to solving for an optimized $q(t)$ time history, the algorithm is used to test for the existence and uniqueness of an arbitrary continuous or impulsive solution to the given boundary value problem. With a well-posed question, the algorithm is then used to search for a $q(t)$ time history. 


\subsubsection{Existence and Non-Uniqueness of a Continuous Solution}

For there to be a physical explanation for the anomaly, a continuous solution must exist for the boundary-value problem. With $\boldsymbol{X}_{2}{ }^{*}$ as the observed post-encounter state and $\boldsymbol{X}_{2}{ }^{0}$ as the predicted post-encounter state, the optimization can determine whether any physically realizable solution exists (regardless of the cause). For this analysis, three $6^{\text {th }}$-order Fourier series aligned with the $\hat{r}, \hat{\phi}$, and $\hat{\theta}$ directions model the unknown acceleration. Setting the sum of the cosine terms in the Fourier series to zero ensures that the applied force is zero at the boundary points. This boundary condition reflects the fact that the anomaly occurs within the data blackout period and that the forces are known both before and after the blackout. The optimizer solves for 39 coefficients. Here, the longest period $\tau$ is set to the length of the full data blackout period.

The optimization algorithm converges to a solution with $J=10^{-5}$. This solution, shown in Figure 7.8, is not unique. There are multiple qualitatively different acceleration time histories that each reproduces the anomalous orbit change. Therefore, a physical explanation may exist. Furthermore, the direction of this force throughout the blackout period cannot be determined exactly from the information given. It is possible that using this technique on other examples of the flyby anomaly can identify common trends among multiple spacecraft that may restrict the direction of the force. However, that issue is not pursued further in this study. 

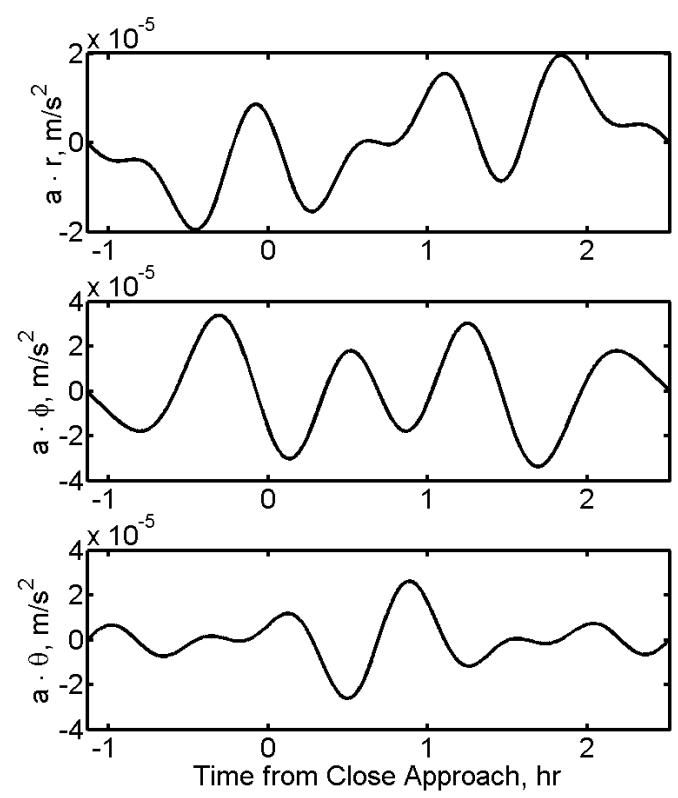

Figure 7.8. Sample continuous acceleration that reproduces the anomaly, shown in $\hat{r}-$, $\hat{\phi}$-, and $\hat{\theta}$-aligned components.

\subsubsection{Possibility of a Single-Impulse Solution}

Using the boxcar basis function instead of the more general Fourier series establishes whether or not an impulse can explain the anomaly. The simulation for this case uses three such functions with $\Delta t=10 \mathrm{~s}$ to represent components in the $\hat{r}, \hat{\phi}$, and $\hat{\theta}$ directions. The optimizer varies three scalar parameters that define the magnitude and direction for each coordinate. The algorithm varies these three parameters in an effort to minimize the cost at the final state. The optimizer also includes a fourth parameter, the impulse start time. The optimizer runs for an array of start times spanning the blackout period, each two minutes apart.

The optimizer settles on impulses whose magnitudes are about $10^{-4}$ to $10^{-3} \mathrm{~m} / \mathrm{s}^{2}$. The resulting costs, each associated with a single impulse at each time point, are shown in Figure 7.9. They are decidedly nonzero, well above the error in the tracking. This result demonstrates that the unknown anomalous acceleration cannot be 
attributed to a single impulsive event at some time in the blackout period. As a result of this analysis, the evaluation of the Lorentz force can neglect single-impulse $q(t)$ time histories and focuses instead on continuous functions that can be approximated by the truncated Fourier series.

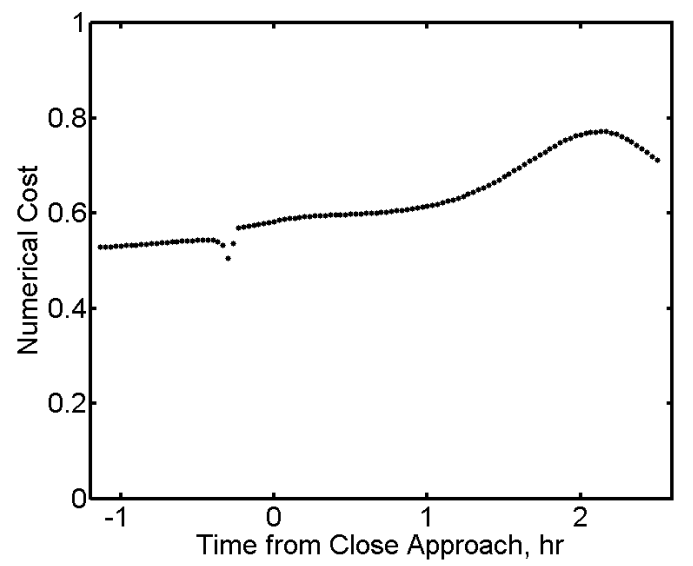

Figure 7.9. Numerical cost at each optimized impulse time-point.

\subsubsection{Optimized Charge Time History}

The optimization routine was initialized with each of the sample functions illustrated in Figure 7.6 (units of $\pm 10 \mathrm{mC} / \mathrm{kg}$ ) as well as a zero vector. Each routine converges to a solution with similar features, especially near close approach. The solution space was evaluated for 24 parameter variations, including of $\tau$ between $1 \mathrm{hr}$ and $8 \mathrm{hr}$ and $n$ between 8 and 32. Each optimization run converged to a solution with $J=0.24$, suggesting that none fully reproduces the anomaly. Figure 7.10 shows one such optimized $q(t) / m$ time history using both the maximum values of $\tau$ and $n$. Figure 7.11 shows the corresponding acceleration in spherical coordinates. The individual state errors are normalized to $\boldsymbol{\rho}^{\boldsymbol{0}}$ in Table 7.3 , such that each value represents the fraction of anomalous error remaining after the charge time history has been applied. 
The error in $z$ clearly dominates the cost, with the supplied force accounting for only $50 \%$ of the anomalous change. To date, use of these algorithms and a Fourier basis function has not produced a more optimized solution.

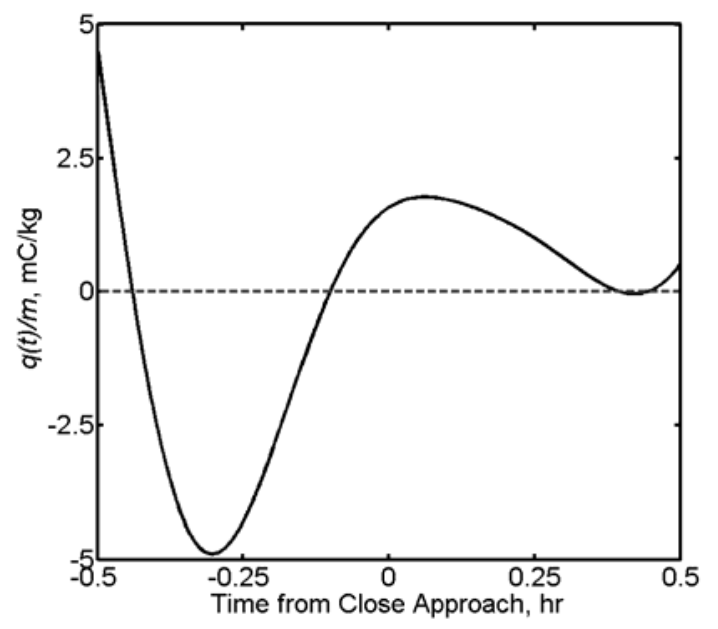

Figure 7.10. Sample optimized $q(t) / m$ time-history
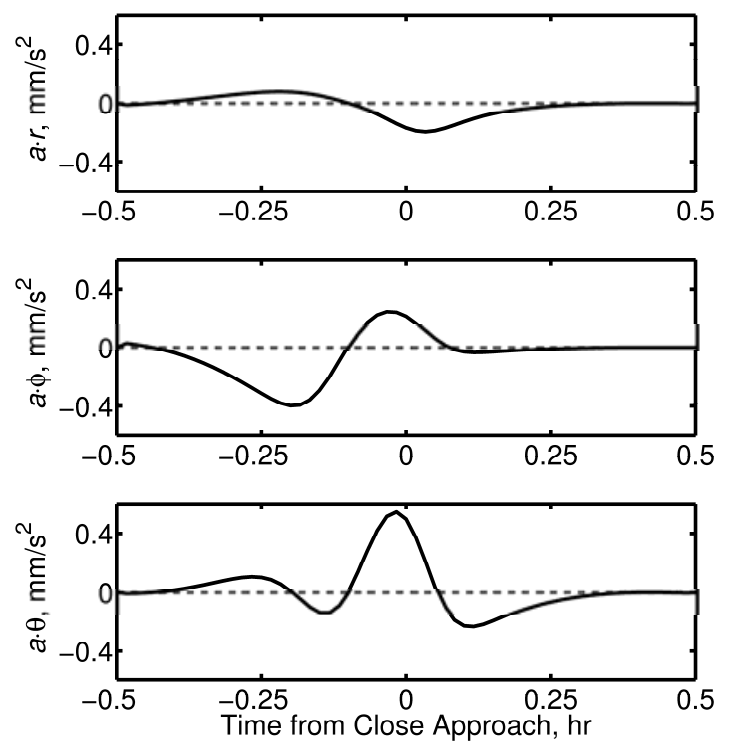

Figure 7.11. Sample optimized Lorentz accelerations, shown in $\hat{r}-, \hat{\phi}-$, and $\hat{\theta}$-aligned components. 
Table 7.3. Normalized State Errors

\begin{tabular}{ccc}
\hline \hline State & & $\begin{array}{c}\text { Normalized } \\
\text { Error }\end{array}$ \\
\hline$x$ & $-7.4 \mathrm{~m}$ & $8.4 \%$ \\
$y$ & $-14.5 \mathrm{~m}$ & $8.5 \%$ \\
$z$ & $0.2 \mathrm{~m}$ & $0.3 \%$ \\
$\dot{x}$ & $0.7 \mathrm{~mm} / \mathrm{s}$ & $5.6 \%$ \\
$\dot{y}$ & $1.5 \mathrm{~mm} / \mathrm{s}$ & $20.7 \%$ \\
$\dot{z}$ & $24.0 \mathrm{~mm} / \mathrm{s}$ & $54.3 \%$ \\
\hline \hline
\end{tabular}

\subsection{Conclusions}

The Lorentz force satisfies all of the known properties of the Earth flyby anomaly. It is physical, non-conservative, bi-directional, distance-dependant, and dependent on declination. This research ignores the specific mechanisms, by which a spacecraft could develop significant charge, and instead treats charge as an output of the analysis. Focusing on the NEAR spacecraft's flyby, perturbation methods are employed to show that the required charge on the spacecraft would need to achieve both positive and negative values to produce the observed trajectory change.

Continuing with the NEAR flyby, numerical methods are used to search for a candidate charge time history. The solutions produced by this search can account for the orbit changes in some directions, but ultimately no more than $75 \%$ of the change in an overall, vector-magnitude sense. Though there exist continuous acceleration time histories that satisfy the observed state change, they cannot be associated with the Lorentz force alone. The optimized charge time histories cannot account for the state error in the $z$ velocity. Therefore, it is unlikely that the anomaly can be attributed to an 
interaction of spacecraft charging with the geomagnetic field. It turns out that $v_{\text {inf }}$ can be matched arbitrarily well if this single scalar is used as the only cost. The same is true for most other scalar cost functions, such as angular-momentum magnitude and energy in various frames. The reason is that the scalar nature of such a parameter obscures the more subtle vector mechanics of the problem. Therefore, the authors recommend that future work on the flyby anomaly consider the full orbital state to prevent false positives.

Although this study focuses on the Lorentz force, it makes at least two other more general contributions to the study of the flyby anomaly. First, the analysis allows one to conclude that the anomalous acceleration cannot be associated with a single impulse. Second, the numerical methodology described here is applicable to any physical phenomenon that can be represented as a time history for use in a simulation environment. It is likely that an exhaustive reevaluation of the proposed causes of this anomaly using such a method, correlated across the many examples, will reveal more about the anomaly than is currently known.

\subsection{Acknowledgments}

The authors wish to thank John D. Anderson for providing insight and ephemerides throughout this research. 


\section{REFERENCES}

[1] Edwards, C., Anderson, J., Beyer, P., Bhaskaran S., Border, J., DiNardo, S., et al, "Tracking Galileo at Earth-2 Perigee Using the Tracking and Data Relay Satellite System," Advances in the Astronautical Sciences, AAS 93-685, Vol. 85, 1993, pp. 1609-1620.

[2] Anderson, J.D., Campbell, J.K., Ekelund, J.E. , Ellis, J., and Jordan, J.F., "Anomalous Orbital-Energy Changes Observed during Spacecraft Flybys of Earth," Physical Review Letters, Vol. 100, 7 March 2008, pp. 091102 1-4. DOI:10.1103/PhysRevLett.100.091102

[3]. Anderson, J.D., J.K. Campbell, and M.M. Nieto, "The Energy Transfer Process in Planetary Flybys," New Astronomy, Vol 12, No 5, July 2007, pp. 383-397. DOI:10.1016/j.newast.2006.11.004

[4] Lammerzahl, C., Preuss, O. , and Dittus, H., "Is the Physics within the Solar system really understood?", Lasers, Clocks, and Drag-Free Control, Springer, Berlin, 2008, pp. 75-98.

[5] Turyshev, S.G., and V.T. Toth, "The Puzzle of the Flyby Anomaly" Space Science Review, August 2008, pp 1-6. DOI: 10.1007/s11214-009-9571-0

[6] Antreasian, P.G., and Guinn, J.R., "Investigations into the Unexpected Delta-V Increases During the Earth Gravity Assists of Galileo and NEAR," AIAA/AAS Astrodynamics Specialist Conference and Exhibit, AIAA 98-4287, Boston, August 1998.

[7] Hasse, W., Birsin, E., and P. Hähnel, "On force-field models of the spacecraft flyby anomaly”, arXiv:0903.0109v1, February 2009.

[8] Lewis, R.A., "Field Theory Model of the Flyby Anomaly", Space Propulsion and Energy Sciences International Forum, American Institute of Physics Conference Proceedings, Vol. 1103, 2009. pp. 226-234. DOI: 10.1063/1.3115499

[9] Busack, H.J., "Simulation of the Flyby Anomaly by Means of an Empirical Asymmetric Gravitational Field with Definite Spatial Orientation," arXiv:0711.2781, November 2007. 
[10] Iorio, L., "The Effect of General Relativity on Hyperbolic Orbits and its Application to the Flyby Anomaly," Scholarly Research Exchange, Vol 2009, Article ID 807695, 2009, pp. 1-8. DOI: 10.3814/2009/807695

[11] Cahill, R.T., "Resolving Spacecraft Earth-Flyby Anomalies with Measured Light Speed Anisotropy", Progress in Physics, Vol. 3, 2008, pp. 9-15.

[12] Cahill, R.T, "Combining NASA/JPL One-Way Optical-Fiber Light-Speed Data with Spacecraft Earth-Flyby Doppler-Shift Data to Characterise 3-Space Flow," Progress in Physics, Vol 4, October 2009, pp. 50-64.

[13] Mbelek, J.P., "Special Relativity May Account for the Spacecraft Flyby Anomalies", arXiv:0809.1888v2, September 2008.

[14] Gerrard, M.B., and T.J. Sumner, "Earth Flyby and Pioneer Anomalies," arXiv:0807.3158v2, October 2008.

[15] Adler, S.L., "Can the Flyby Anomaly be Attributed to Earth-Bound Dark Matter?," Physical Review D, Vol 79, No 2, 2009, pp. 023505-1-10. DOI: 10.1103/PhysRevD.79.023505

[16] Adler, S.L., "Modeling the Flyby Anomalies with Dark Matter Scattering", arXiv:0908.2414v2, August 2009.

[17] McCulloch, M.E., "Modeling the Flyby Anomalies Using a Modification of Inertia," Monthly Notices of the Royal Astronomical Society: Letters, Vol 289, No 1, July 2008, pp. L57-L60. DOI:10.1111/j.1745-3933.2008.00523.x

[18] McCulloch, M.E., "Can the Flyby Anomalies be Explained by a Modification of Inertia," Journal of the British Interplanetary Society, Vol 61, September 2008, pp. 373-378.

[19] Petry, W., "A Possible Exlanation of Anomalous Earth Flybys", arXiv:0806.0334v1, June 2008.

[20] Grun, E., Kruger, H., Graps, A.L., Hamilton, D.P., Heck, A., et al, "Galileo Observes Electromagnetically Coupled Dust in the Jovian Magnetosphere", Journal of Geophysical Research, Vol. 103, No. E9, 1998, pp. 20011-20022.

[21] Colwell, J.E., Horanyi, M., and Grun, E., "Capture of Interplanetary and Interstellar Dust by the Jovian Magnetosphere", Science, Vol. 280, April 1998, pp. 88-91. DOI: $10.1126 /$ science. 280.5360 .88 
[22] Schaffer, L. and Burns, J.A., "The Dynamics of Weakly Charged Dust: Motion through Jupiter's Gravitational and Magnetic Fields," Journal of Geophysical Research, Vol. 92, 1987, pp. 2264-2280. DOI: 10.1029/JA092iA03p02264

[23] Horanyi, M., "Charged Dust Dynamics in the Solar System," Annual ReviewsAstronomy and Astrophysics, Vol. 34, 1996, pp. 383-418. DOI:

10.1146/annurev.astro.34.1.383

[24] Smith, B.A., Soderblom, L.A. , Beebe, R.F. , Boyce, J., Briggs, G.A., et al., 1982. "Encounter with Saturn: Voyager 1 Imaging Science Results," Science, Vol. 212, No. 4491, 1982, pp. 163-191. DOI: 10.1126/science.212.4491.163

[25] Streetman, B. and M. Peck, "New Synchronous Orbits Using the Geomagnetic Lorentz Force," Journal of Guidance, Control, and Dynamics, Vol 30, No 6, 2007, pp. 1677-1690. DOI: 10.2514/1.29080

[26] Streetman, B. and M. Peck, "A General Bang-Bang Control Method for Lorentz Augmented Orbits," AAS 08-111, AAS Spaceflight Mechanics Meeting, Galveston, TX, Jan 27-31, 2008, pp. 1-18.

[27] Sumner, T., Araujo, H., Davidge, D., Howard, A., Lee, C., Rochester, G., Shaul, D., and Wass, P., "Description of Charging/ Discharging Process of the LISA Sensors". Classical and Quantum Gravity, Vol. 21, No. 5, March 72004 , pp.S597-S602.DOI: 10.1088/0264-9381/21/5/031

[28] Atchison, J.A. and Peck, M., "Lorentz Augmented Jovian Orbit Insertion." Journal of Guidance, Control, and Dynamics, Vol. 32, No. 2, March 2009, pp. 418-423. DOI: $10.2514 / 1.38406$

[29] Peck, M., Streetman, B., Saaj, C.M., and Lappas, V., "Spacecraft Formation Flying Using Lorentz Forces," Journal of the British Interplanetary Society, Vol. 60, July 2007, pp 263-267.

[30] Streetman, B. and M. Peck, "Gravity-Assist Maneuvers Augmented by the Lorentz Force," Journal of Guidance, Control, and Dynamics, Vol 32, No 5, 2009, pp. 1639-1647. DOI: 10.2514/1.35676

[31] Vallado, D. A., "Chapter 9: General Perturbation Techniques", Fundamentals of Astrodynamics and Applications, 2nd Ed., Microcosm Press, El Segundo, CA, 2004, pp. 567-669. 


\section{CHAPTER 8 CONCLUSIONS}

\subsection{Overview of Length Scaling in Spacecraft Dynamics}

The overarching objective of this research is to identify and characterize the effects of characteristic length on spacecraft orbit and attitude dynamics. The analysis begins with a survey of non-gravitational accelerations acting on a spacecraft in the Near Earth environment. After identifying the accelerations' dependence on length, the analysis compares their magnitude across length-scales and altitudes. This investigation offers a number of contributions, including:

- Connections between the orbital behavior of spacecraft and interplanetary dust

- Guidelines for what a given simulation must include for a given spacecraft length scale and altitude

- Classification of altitude and length-scale "regimes," within which the orbital and attitude dynamics are dominated by a single non-gravitational acceleration

- Identification of spacecraft length scales that offer non-Keplerian dynamics

- Identification of candidate missions for a millimeter-scale spacecraft These findings motivate deeper analysis of specific acceleration models, namely solar radiation pressure, aerodynamic drag, and the Lorentz force. Each of these cases is considered in terms of mission concepts and architectures for a millimeter-scale spacecraft. 


\subsection{Solar Radiation Pressure}

Photon pressure from the sun dominates the dynamics of small spacecraft at high altitudes. This pressure has been proposed as a means of propellantless propulsion for decades. However, it has yet to be fully employed, due to a number of critical challenges. This research suggests that a millimeter-scale spacecraft architecture could overcome these challenges and offer a feasible demonstration. It presents the first-ever design in which the spacecraft bus, in this case an integrated circuit microchip, performs as the solar sail with no additional deployments or membranes. The research addresses the full orbital and attitude dynamics for this microchip architecture, producing a number of key outcomes. It is the first-ever survey and identification of orbital and mission applications for a sun-pointing, passive solar sail. It offers two new, passive, sun-pointing attitude architectures, with local and/or global stability. Finally, it develops a set of missions and applications for this candidate solar sail, assessing feasibility and performance. This research suggests that near-term solar sailing missions may be more easily achieved by small sails than by typical solar sail designs.

\subsection{Atmospheric Drag}

Atmospheric-drag accelerations dominate the orbital and attitude dynamics of a small spacecraft at the lowest altitudes. Drag accelerations act in the anti-velocity direction, removing kinetic energy from the orbit. This behavior has applications in passive attitude control, spacecraft de-orbiting, or controlled re-entry. This research focuses on re-entry because of additional heat-transfer benefits associated with length scaling. Hypersonic forced convection typically results in the demise of a re-entering spacecraft. This analysis finds that the same scaling that drives the magnitude of drag 
acceleration for small spacecraft, area-to-mass, also drives the thermal loading for a small spacecraft. For the right geometry, it may be possible to re-enter a millimeterscale spacecraft passively, such that it never attains the high temperatures associated with ablation. In fact, the temperatures may stay low enough to allow on-board electronics to continuously operate.

\subsection{The Lorentz Force}

The Lorentz force can dominate the dynamics of a spacecraft with a very high charge-to-mass ratio in the near-Earth environment. This critical quantity is especially sensitive to length scale, varying with the inverse of the square of characteristic length. This research also finds that plasma charging mechanisms are strongly dependent on characteristic size. The larger a body, the more power is required to offset the currents associated with plasma interactions. These two effects suggest that a millimeter-scale spacecraft may be the most feasible Lorentz-propelled system. The research describes an architecture intended to be the first ever demonstration of the effects of the Lorentz force on an artificial satellite. The design includes a mechanism for achieving a net electrostatic charge using only solar cells and two filament plasma contactors. Additionally, this research focuses on two applications of the Lorentz force, which contribute to the general study of Lorentz augmented orbits.

\subsubsection{Jovian Orbit Insertion}

This analysis considers what is thought to be one of the lowest charge-to-mass applications for near term Lorentz spacecraft, orbit insertion at Jupiter. Jupiter’s large magnetic field and fast rate of rotation mean that the near Jupiter environment is well suited for the Lorentz force. The analysis considered energy and power in the context 
of the planetary capture and orbital circularization problem. The key contribution from this research is a numerical analysis that relates the outcome for a spacecraft of a given charge-to-mass ratio and initial Jovian approach orbit. Using a time-optimal bang-off charge controller, certain combinations of these parameters yield successful captures and circularization, while others result in escape or planetary crashes. These plots therefore offer insight into some of the subtleties of Lorentz propulsion. They also offer guidelines for spacecraft designers interested in incorporating such a maneuver into the mission design.

\subsubsection{Lorentz Accelerations in the Earth Fly-by Anomaly}

In the past two decades, six spacecraft have experienced inexplicable accelerations while executing Earth gravity assists. These accelerations have come to be known as the Earth fly-by anomaly. This research explores whether the anomaly is associated with the Lorentz force. It approaches the question in terms of the full six degree-offreedom state vector and is the first analysis to do so. A survey of the available literature on the anomaly identifies six key characteristics of the anomaly, all of which apply to a modern understanding of the Lorentz force in the orbital mechanics problem. The analysis focuses on the Near Earth Asteroid Rendezvous spacecraft, employing both perturbation techniques and numerical methods. The primary outcomes of this research are:

- Elimination of the possibility of a single impulse as the source the anomaly on the NEAR spacecraft

- Demonstration that there exist continuous acceleration time-histories that can reproduce the anomaly 
- Development of a numerical optimization routine designed to solve for an unknown acceleration magnitude time-history, given a known direction

- Demonstration that the net electrostatic charge on the NEAR spacecraft would have to be negative at some point to cause the correct polarity of change in the anomaly due to the Lorentz force

- Finally, elimination of the Lorentz force as a candidate for the source of the anomaly.

The key outcome of this analysis is that the anomaly cannot be associated with the Lorentz force. That is, there does not exist a charge time-history that can reproduce the observed anomaly. This conclusion eliminates a likely candidate for the anomaly, and narrows the list of remaining proposed explanations.

\subsection{Millimeter-Scale Spacecraft Design}

Each of these analyses offers a millimeter-scale spacecraft bus design. This design uses a thin square plate of silicon as the standard geometry. The most recent design, dubbed "Sprite," is discussed in the analysis of aerodynamic drag. The current iteration of Sprite is a printed-circuit board module with all of the expected functionality. The next iteration, being developed in collaboration with Sandia National Laboratories is a multi-chip module. These development efforts justify research into small spacecraft dynamics by demonstrating functionality at millimeterscales.

The communications subsystem drives these designs. The spacecraft must complete a long-distance communications link with very low available power. The solution proposed here involves two mechanisms. First, the circuitry operates in a "bursty" mode, in that it transmits only when it has sufficient power. The remainder 
of the time is spent collecting power in preparation for the next transmission. Second, the communications protocol incorporates convolutional filtering, which offers a large increase in effective link gain, with little additional spacecraft hardware. This method takes advantage of signal processing at the ground station.

\subsection{Future Directions}

This research represents a foundation for future millimeter-scale spacecraft efforts. There are a number of relevant directions to focus future efforts:

- This research has focused solely on environmental accelerations; it could be beneficial to consider length-scaling in the context of engineered propulsion techniques.

- Millimeter-scale solar sails would benefit from a form of active attitude control. Such a technology would enable much more effective energychange maneuvers and would allow the full benefits of solar radiation pressure to be realized.

- The heliocentric orbital mechanics of a spinning fixed-attitude millimeterscale solar sail may offer feasible, meaningful mission opportunities. Initial investigations suggest that such a spacecraft could execute inclination and even energy change maneuvers with no active control.

- Further aerothermal modeling and testing for the millimeter-scale architecture may offer greater insight into the re-entry thermal environment and approaches to yield more effective sensors.

- As new results from plasma charging tests become available, the Lorentzpropelled spacecraft design should be reassessed and optimized. These 
efforts appear to be the most direct path to demonstrating Lorentz augmented orbital mechanics.

- The millimeter-scale spacecraft prototype is deserving of future efforts and developments. In particular, initial studies suggest that it could incorporate a variety of meaningful sensors, offering new distributed science missions.

- This research introduces research questions associated with the exfiltration of data from large networks of independent, autonomous sensors. The solution must account for irregular or simultaneous transmissions from lowpower sensors distributed across large distances. 